\title{
Synthesis of the GHIJKL Fragment of Gymnocin-B
}

Takeo Sakai*, Hideaki Sakakibara, Yumi Omoto, Marina Tsunekawa, Yoshinori Hadano, Shota Kato, and Yuji Mori*

Faculty of Pharmacy, Meijo University, 150 Yagotoyama, Tempaku-ku, Nagoya 468-8503, Japan sakait@meijo-u.ac.jp; mori@meijo-u.ac.jp

Table of Contents

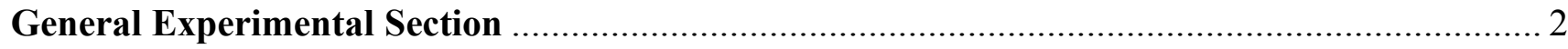

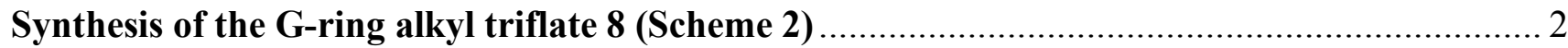

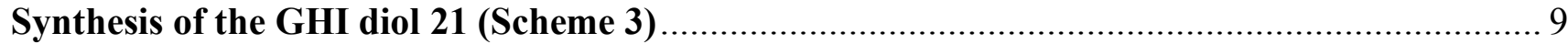

Synthesis of the seven-membered model ketone 22 ……......................................................... 22

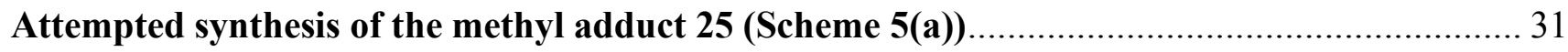

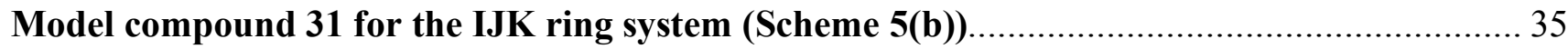

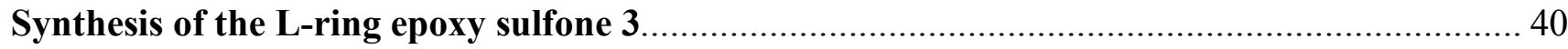

Synthesis of the GHIJKL fragment 1 (Scheme 6) ……............................................................... 44

Comparison of ${ }^{1} \mathrm{H}$ and ${ }^{13} \mathrm{C}$ spectra between the GHIJKL fragment 1 and gymnocin-B............ 54

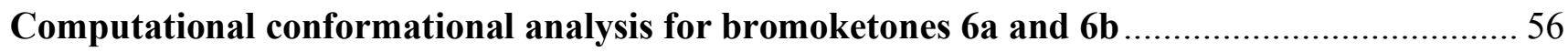

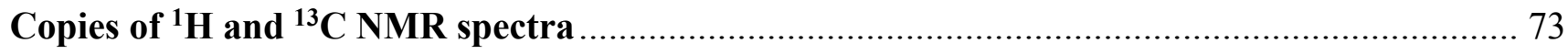




\section{General Experimental Section}

All air- and moisture-sensitive reactions were carried out under an argon atmosphere in commercially available dry solvents under anhydrous conditions. The term "dried" refers to the drying of an organic solution over $\mathrm{MgSO}_{4}$ followed by filtration. Flash chromatography was carried out with silica gel (spherical, neutral, particle size $40-50 \mathrm{~mm}$ ). $m$-CPBA was purified prior to use by dissolving benzene, washing with $\mathrm{pH} 7.4$ phosphate buffer, and concentration under reduced pressure. Chemical shifts are reported in ppm relative to the internal TMS $\left(\delta 0.00 \mathrm{ppm}\right.$ for $\left.\mathrm{CDCl}_{3}\right)$ for ${ }^{1} \mathrm{H} \mathrm{NMR}$ spectrum and to the solvent signals $\left(\delta 77.0 \mathrm{ppm}\right.$ for $\left.\mathrm{CDCl}_{3}\right)$ for ${ }^{13} \mathrm{C}$ NMR spectrum. Data are reported as follows: chemical shift, integration, multiplicity $(\mathrm{s}=$ singlet, $\mathrm{d}=$ doublet, $\mathrm{t}=$ triplet, $\mathrm{q}=$ quartet, $\mathrm{m}=$ multiplet, br $=$ broad). High-resolution mass spectra were recorded on a magnetic sector FAB mass spectrometer, a magnetic sector EI mass spectrometer, or an orbitrap DART mass spectrometer.

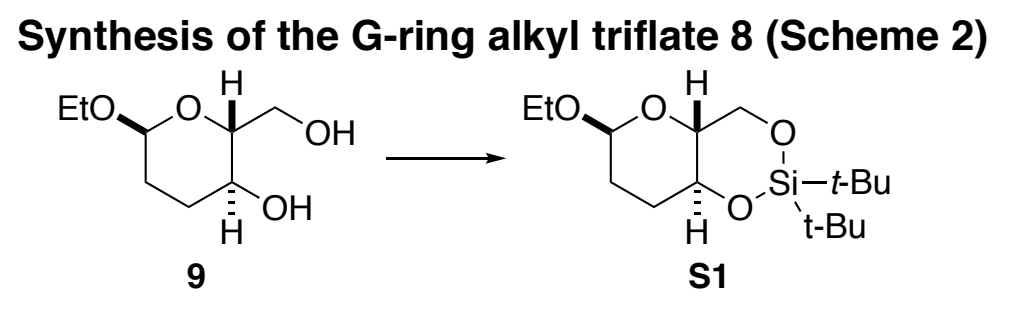

Silylene S1. To a solution of diol $\mathbf{9}^{1}$ (5.80 g, $32.9 \mathrm{mmol}, 88: 12$ mixture of $\beta: \alpha$ isomers) in DMF (58 $\mathrm{mL})$ and 2,6-lutidine $\left(11.5 \mathrm{~mL}, 98.8 \mathrm{mmol}, 3.0\right.$ equiv) at $0{ }^{\circ} \mathrm{C}$ was added $t$-Bu $\mathrm{Bu}_{2} \mathrm{Si}(\mathrm{OTf})_{2}(17.7 \mathrm{~mL}, 49.4$ mmol, 1.5 equiv). The reaction mixture was stirred at $0^{\circ} \mathrm{C}$ for $1 \mathrm{~h}$, and the reaction was quenched with saturated aqueous $\mathrm{NaHCO}_{3}$ solution. The mixture was extracted with EtOAc/Hex (1:1), and the organic layer was washed with water and brine, dried, and concentrated under reduced pressure. Flash chromatography on silica gel (6\% EtOAc in $n$-hexane) afforded silylene $\mathbf{S 1}(9.24 \mathrm{~g}, 89 \%)$ as a colorless oil. 88:12 mixture of $\beta: \alpha$ isomers; $[\alpha]^{27} \mathrm{D}+59.8\left(c 0.99, \mathrm{CHCl}_{3}\right)$; IR (film) 2961, 2933, $28601104 \mathrm{~cm}^{-1}$; ${ }^{1} \mathrm{H}$ NMR for the major $\beta$-isomer $\left(600 \mathrm{MHz}, \mathrm{CDCl}_{3}\right) \delta 4.73(1 \mathrm{H}, \mathrm{d}, J=3.3 \mathrm{~Hz}), 4.01(1 \mathrm{H}, \mathrm{dd}, J=10.0$, $4.7 \mathrm{~Hz}), 3.82(1 \mathrm{H}, \mathrm{t}, J=10.2 \mathrm{~Hz}), 3.78(1 \mathrm{H}, \mathrm{ddd}, J=10.3,9.2,4.4 \mathrm{~Hz}), 3.71(1 \mathrm{H}, \mathrm{m}), 3.70(1 \mathrm{H}, \mathrm{dq}, J=$ 9.8, 7.1 Hz), $3.45(1 \mathrm{H}, \mathrm{dq}, J=9.8,7.1 \mathrm{~Hz}), 1.89(1 \mathrm{H}, \mathrm{m}), 1.85-1.80(2 \mathrm{H}, \mathrm{m}), 1.77(1 \mathrm{H}, \mathrm{m}), 1.23(3 \mathrm{H}, \mathrm{t}, J$ 
$=7.1 \mathrm{~Hz}), 1.05(9 \mathrm{H}, \mathrm{s}), 1.00(9 \mathrm{H}, \mathrm{s}) ;{ }^{13} \mathrm{C}$ NMR for the major $\beta$-isomer $\left(150 \mathrm{MHz}, \mathrm{CDCl}_{3}\right) \delta 96.1,73.9$, 68.7, 67.3, 62.5, 29.5, 27.5, 27.4, 27.1, 22.7, 19.9, 15.1; ${ }^{1} \mathrm{H}$ NMR for the minor $\alpha$-isomer $(600 \mathrm{MHz}$, $\left.\mathrm{CDCl}_{3}\right) \delta 4.51(1 \mathrm{H}, \mathrm{dd}, J=9.5,2.2 \mathrm{~Hz}), 4.08(1 \mathrm{H}, \mathrm{dd}, J=10.2,4.9 \mathrm{~Hz}), 3.91(1 \mathrm{H}, \mathrm{t}, J=10.3 \mathrm{~Hz}), 3.90$ $(1 \mathrm{H}, \mathrm{dq}, J=9.5,7.1 \mathrm{~Hz}), 3.78(1 \mathrm{H}, \mathrm{m}), 3.52(1 \mathrm{H}, \mathrm{dq}, J=9.5,7.1 \mathrm{~Hz}), 3.36(1 \mathrm{H}, \mathrm{ddd}, J=10.3,9.2,5.0$ Hz), $2.12(1 \mathrm{H}, \mathrm{dq}, J=12.8,3.9 \mathrm{~Hz}), 1.74(1 \mathrm{H}, \mathrm{m}), 1.60(1 \mathrm{H}, \mathrm{ddd}, J=13.5,9.2,3.7 \mathrm{~Hz}), 1.52$ (1H, dddd, $J=13.7,12.8,10.4,3.6 \mathrm{~Hz}), 1.22(3 \mathrm{H}, \mathrm{t}, J=7.2 \mathrm{~Hz}), 1.04(9 \mathrm{H}, \mathrm{s}), 0.99(9 \mathrm{H}, \mathrm{s}) ;{ }^{13} \mathrm{C}$ NMR for the minor $\alpha$-isomer $\left(150 \mathrm{MHz}, \mathrm{CDCl}_{3}\right) \delta 101.7,74.8,73.2,66.9,64.6,31.2,30.7,27.5,27.1,22.7,19.9,15.1$; HRDARTMS $m / z$ calcd for $\mathrm{C}_{16} \mathrm{H}_{33} \mathrm{O}_{4} \mathrm{Si}\left(\mathrm{MH}^{+}\right)$317.2143, found 317.2137.

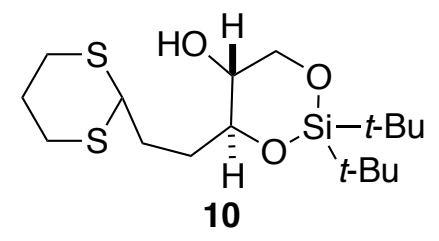

Dithioacetal 10. To a solution of ethyl acetal S1 (9.22 g, $29.1 \mathrm{mmol})$ and 1,3-propanedithiol (3.22 $\mathrm{mL}$, $32.1 \mathrm{mmol}, 1.1$ equiv) in $\mathrm{CH}_{2} \mathrm{Cl}_{2}(146 \mathrm{~mL})$ at $0{ }^{\circ} \mathrm{C}$ was added $\mathrm{BF}_{3} \cdot \mathrm{OEt}_{2}(4.10 \mathrm{~mL}, 32.1 \mathrm{mmol})$, and the mixture was stirred at $0{ }^{\circ} \mathrm{C}$ for $1 \mathrm{~h}$. The reaction was quenched with $10 \%$ aqueous $\mathrm{NaOH}$ solution and the resulting mixture was extracted with $\mathrm{Et}_{2} \mathrm{O}$. The extract was washed with $10 \%$ aqueous $\mathrm{NaOH}$ solution and brine, dried, and concentrated under reduced pressure. Flash chromatography on silica gel $(30 \%$ EtOAc in $n$-hexane) afforded alcohol $10(11.1 \mathrm{~g}, 100 \%)$ as a colorless oil. $[\alpha]^{26}-35.3\left(c 1.25, \mathrm{CHCl}_{3}\right)$; IR (film) 3422, 2931, 2858, $1062 \mathrm{~cm}^{-1} ;{ }^{1} \mathrm{H}$ NMR (600 MHz, $\left.\mathrm{CDCl}_{3}\right) \delta 4.13(1 \mathrm{H}, \mathrm{t}, J=6.7 \mathrm{~Hz}), 4.07(1 \mathrm{H}$, $\mathrm{dd}, J=10.5,4.7 \mathrm{~Hz}), 3.79(1 \mathrm{H}, \mathrm{td}, J=8.8,2.2 \mathrm{~Hz}), 3.77(1 \mathrm{H}, \mathrm{t}, J=10.3 \mathrm{~Hz}), 3.53(1 \mathrm{H}, \mathrm{dddd}, J=10.3$, 8.8, 6.1, $4.7 \mathrm{~Hz}), 2.91-2.82(4 \mathrm{H}, \mathrm{m}), 2.15-2.05(3 \mathrm{H}, \mathrm{m}), 1.95(1 \mathrm{H}, \mathrm{d}, J=6.1 \mathrm{~Hz}), 1.91-1.84(2 \mathrm{H}, \mathrm{m}), 1.64$ $(1 \mathrm{H}, \mathrm{m}), 1.04(9 \mathrm{H}, \mathrm{s}), 0.98(9 \mathrm{H}, \mathrm{s}) ;{ }^{13} \mathrm{C} \mathrm{NMR}\left(150 \mathrm{MHz}, \mathrm{CDCl}_{3}\right) \delta$ 77.9, 70.4, 68.5, 47.7, 31.5, 30.9, 30.41, 30.38, 27.5, 27.0, 26.0, 22.6, 19.9; HRDARTMS $m / z$ calcd for $\mathrm{C}_{17} \mathrm{H}_{35} \mathrm{O}_{3} \mathrm{~S}_{2} \mathrm{Si}\left(\mathrm{MH}^{+}\right)$379.1791, found 379.1789. 


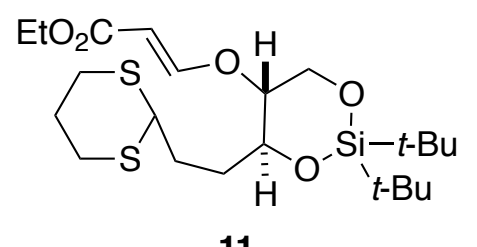

11

Unsaturated ester 11. To a solution of alcohol 10 (10.3 g, $27.3 \mathrm{mmol})$ in $\mathrm{CH}_{2} \mathrm{Cl}_{2}(140 \mathrm{~mL})$ were added ethyl propiolate $(8.40 \mathrm{~mL}, 81.8 \mathrm{mmol}, 3.0$ equiv) and $N$-methylmorpholine $(9.00 \mathrm{~mL}, 81.8 \mathrm{mmol}, 3.0$ equiv). The reaction mixture was stirred at room temperature for $40 \mathrm{~min}$, and the reaction was quenched with saturated aqueous $\mathrm{NH}_{4} \mathrm{Cl}$ solution. The mixture was extracted with EtOAc and the extract was washed with brine, dried, and concentrated under reduced pressure. Flash chromatography on silica gel $(6 \rightarrow 10 \%$ EtOAc in $n$-hexane) afforded the unsaturated ester 11 (11.5 g, 88\%) as a colorless solid. Mp $113-114{ }^{\circ} \mathrm{C} ;[\alpha]^{26} \mathrm{D}-15.9\left(c 0.98, \mathrm{CHCl}_{3}\right) ; \mathrm{IR}$ (film) 2933, 2896, 2859, 1712, 1644, $1128 \mathrm{~cm}^{-1} ;{ }^{1} \mathrm{H}$ NMR $\left(600 \mathrm{MHz}, \mathrm{CDCl}_{3}\right) \delta 7.44(1 \mathrm{H}, \mathrm{d}, J=12.3 \mathrm{~Hz}), 5.31(1 \mathrm{H}, \mathrm{d}, J=12.3 \mathrm{~Hz}), 4.17(2 \mathrm{H}, \mathrm{q}, J=7.2 \mathrm{~Hz}), 4.13$ $(1 \mathrm{H}, \mathrm{dd}, J=10.9,4.5 \mathrm{~Hz}), 4.09(1 \mathrm{H}, \mathrm{t}, J=6.9 \mathrm{~Hz}), 3.98(1 \mathrm{H}, \mathrm{td}, J=9.1,2.4 \mathrm{~Hz}), 3.88(1 \mathrm{H}, \mathrm{dd}, J=10.8$ $9.9 \mathrm{~Hz}), 3.74(1 \mathrm{H}, \mathrm{ddd}, J=9.6,8.8,4.5 \mathrm{~Hz}), 2.82-2.90(4 \mathrm{H}, \mathrm{m}), 2.12(1 \mathrm{H}, \mathrm{dddd}, J=14.1,7.2,4.4,2.9$ Hz), 2.07 (1H, dddd, $J=13.6,10.2,7.2,4.7 \mathrm{~Hz}), 1.93(1 \mathrm{H}, \mathrm{m}), 1.81-1.90(2 \mathrm{H}, \mathrm{m}), 1.62(1 \mathrm{H}, \mathrm{dtd}, J=13.4$, 9.9, $4.8 \mathrm{~Hz}), 1.28(3 \mathrm{H}, \mathrm{t}, J=7.2 \mathrm{~Hz}), 1.04(9 \mathrm{H}, \mathrm{s}), 1.00(9 \mathrm{H}, \mathrm{s}) ;{ }^{13} \mathrm{C} \mathrm{NMR}\left(150 \mathrm{MHz}, \mathrm{CDCl}_{3}\right) \delta 167.4$, $161.1,98.8,80.3,75.6,65.2,59.9,47.4,31.5,30.9,30.42,30.37,27.4,27.0,25.9,22.6,20.0,14.3$; HRDARTMS $m / z$ calcd for $\mathrm{C}_{22} \mathrm{H}_{41} \mathrm{O}_{5} \mathrm{~S}_{2} \mathrm{Si}\left(\mathrm{MH}^{+}\right)$477.2159, found 477.2158.

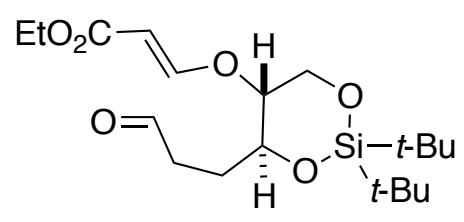

12

Aldehyde 12. To a suspension of dithioacetal 11 (11.5 g, $24.1 \mathrm{mmol})$ and $\mathrm{NaHCO}_{3}(40.5 \mathrm{~g}, 482 \mathrm{mmol}$, 20 equiv) in a mixed solvent of $\mathrm{CH}_{3} \mathrm{CN}$ and water $(4: 1,120 \mathrm{~mL})$ was added $\mathrm{MeI}(30.0 \mathrm{~mL}, 482 \mathrm{mmol}, 20$ equiv). The reaction mixture was stirred at room temperature for $19 \mathrm{~h}$, and the reaction was quenched with saturated aqueous $\mathrm{Na}_{2} \mathrm{~S}_{2} \mathrm{O}_{3}$ solution. After removal of $\mathrm{CH}_{3} \mathrm{CN}$ by a rotary evaporator, the resulting 
mixture was extracted with EtOAc. The extract was washed with water and brine, dried, and concentrated under reduced pressure. Flash chromatography $(10 \rightarrow 20 \%$ EtOAc in $n$-hexane) afforded aldehyde 12 (8.91 g, 96\%) as a colorless oil. $[\alpha]^{26}{ }_{\mathrm{D}}-39.8\left(c \mathrm{1} .89, \mathrm{CHCl}_{3}\right)$; IR (film) 2962, 2934, 2860, 1713, 1644, $1129 \mathrm{~cm}^{-1},{ }^{1} \mathrm{H}$ NMR $\left(600 \mathrm{MHz}, \mathrm{CDCl}_{3}\right) \delta 9.81(1 \mathrm{H}, \mathrm{dd}, J=2.1,1.2 \mathrm{~Hz}), 7.45(1 \mathrm{H}, \mathrm{d}, J=12.5 \mathrm{~Hz}), 5.32$ $(1 \mathrm{H}, \mathrm{d}, J=12.5 \mathrm{~Hz}), 4.17(2 \mathrm{H}, \mathrm{q}, J=7.0 \mathrm{~Hz}), 4.16(1 \mathrm{H}, \mathrm{dd}, J=10.8,4.8 \mathrm{~Hz}), 4.01(1 \mathrm{H}, \mathrm{td}, J=9.3,2.7$ $\mathrm{Hz}), 3.88(1 \mathrm{H}, \mathrm{t}, J=10.5 \mathrm{~Hz}), 3.76(1 \mathrm{H}, \mathrm{ddd}, J=9.9,9.0,4.6 \mathrm{~Hz}), 2.63(1 \mathrm{H}, \mathrm{dtd}, J=17.4,7.0,2.1 \mathrm{~Hz})$, $2.57(1 \mathrm{H}, \mathrm{dtd}, J=17.4,7.0,1.1 \mathrm{~Hz}), 2.11(1 \mathrm{H}, \mathrm{dtd}, J=14.0,6.9,2.7 \mathrm{~Hz}), 1.76(1 \mathrm{H}, \mathrm{ddt}, J=14.0,9.9,7.0$ $\mathrm{Hz}), 1.28(3 \mathrm{H}, \mathrm{t}, J=7.0 \mathrm{~Hz}), 1.03(9 \mathrm{H}, \mathrm{s}), 0.99(9 \mathrm{H}, \mathrm{s}) ;{ }^{13} \mathrm{C} \mathrm{NMR}\left(150 \mathrm{MHz}, \mathrm{CDCl}_{3}\right) \delta 201.9,167.3$, 161.0, 99.0, 80.3, 75.4, 65.3, 60.0, 39.7, 27.5, 27.4, 26.9, 22.5, 19.9, 14.3; HRDARTMS $m / z$ calcd for $\mathrm{C}_{19} \mathrm{H}_{35} \mathrm{O}_{6} \mathrm{Si}\left(\mathrm{MH}^{+}\right)$387.2197, found 387.2196.

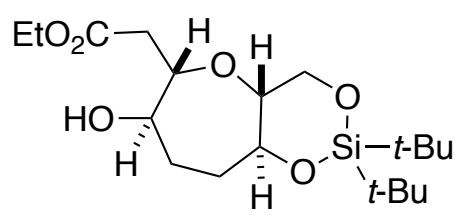

13

Oxepane 13. To a solution of aldehyde $12(8.84 \mathrm{~g}, 22.9 \mathrm{mmol})$ in THF $(115 \mathrm{~mL})$ was added $\mathrm{MeOH}$ (2.8 $\mathrm{mL}, 68.7 \mathrm{mmol}, 3.0$ equiv), and the mixture was cooled to $-20{ }^{\circ} \mathrm{C}$. Samarium iodide (II) (0.1 M solution in THF, $500 \mathrm{~mL}, 50.0 \mathrm{mmol}, 2.2$ equiv) was added dropwise via cannula, and the mixture was stirred for $40 \mathrm{~min}$ at $-20^{\circ} \mathrm{C}$. During this $40 \mathrm{~min}$, the solution color was deep blue, indicating the presence of active samarium iodide (II). The reaction was quenched with saturated aqueous $\mathrm{NaHCO}_{3}$ solution and saturated aqueous $\mathrm{Na}_{2} \mathrm{~S}_{2} \mathrm{O}_{3}$ solution, and the reaction was warmed to room temperature. After removal of THF by a rotary evaporator, the mixture was extracted with EtOAc. The extract was washed with brine, dried, and concentrated under reduced pressure. Flash chromatography on silica gel ( $10 \rightarrow 20 \%$ EtOAc in $n$-hexane) afforded oxepane $13(8.68 \mathrm{~g}, 98 \%)$ as a colorless solid. Mp $81-84{ }^{\circ} \mathrm{C} ;[\alpha]^{25} \mathrm{D}-17.7(c 1.04$, $\mathrm{CHCl}_{3}$ ); IR (film) 3458, 2933, 2859, 1738, $1105 \mathrm{~cm}^{-1} ;{ }^{1} \mathrm{H}$ NMR (600 MHz, $\left.\mathrm{CDCl}_{3}\right) \delta 4.16(2 \mathrm{H}, \mathrm{q}, J=7.2$ $\mathrm{Hz}), 4.01(1 \mathrm{H}, \mathrm{dd}, J=10.2,5.0 \mathrm{~Hz}), 3.81(1 \mathrm{H}, \mathrm{ddd}, J=8.8,7.5,4.6 \mathrm{~Hz}), 3.78(1 \mathrm{H}$, ddd, $J=9.4,6.8,6.0$ 
$\mathrm{Hz}), 3.73(1 \mathrm{H}, \mathrm{dt}, J=7.5,4.2 \mathrm{~Hz}), 3.71(1 \mathrm{H}, \mathrm{t}, J=10.4 \mathrm{~Hz}), 3.46(1 \mathrm{H}, \mathrm{ddd}, J=10.1,9.5,5.0 \mathrm{~Hz}), 2.70$ $(1 \mathrm{H}, \mathrm{dd}, J=16.0,4.6 \mathrm{~Hz}), 2.49(1 \mathrm{H}, \mathrm{dd}, J=16.0,8.8 \mathrm{~Hz}), 2.08(1 \mathrm{H}, \mathrm{d}, J=4.6 \mathrm{~Hz}), 1.96-1.90(2 \mathrm{H}, \mathrm{m})$, 1.90-1.82 (2H, m), $1.27(3 \mathrm{H}, \mathrm{t}, J=7.2 \mathrm{~Hz}), 1.02(9 \mathrm{H}, \mathrm{s}), 0.99(9 \mathrm{H}, \mathrm{s}) ;{ }^{13} \mathrm{C} \mathrm{NMR}\left(150 \mathrm{MHz}, \mathrm{CDCl}_{3}\right) \delta$ 171.6, 82.9, 82.5, 77.8, 74.1, 67.5, 60.7, 40.3, 29.8, 29.7, 27.5, 27.1, 22.5, 20.0, 14.2; HRDARTMS $m / z$ calcd for $\mathrm{C}_{19} \mathrm{H}_{37} \mathrm{O}_{6} \mathrm{Si}\left(\mathrm{MH}^{+}\right)$389.2354, found 389.2354.

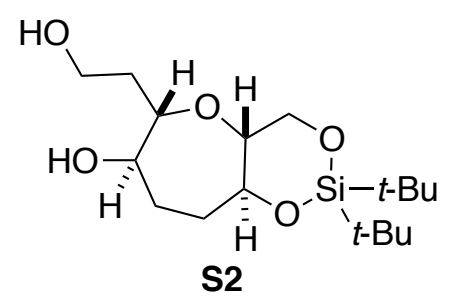

DIOL S2. To a solution of ethyl ester $13(8.69 \mathrm{~g}, 22.4 \mathrm{mmol})$ in THF $(220 \mathrm{~mL})$ was added $\mathrm{LiBH}_{4}(3.0$ M solution in THF, $30 \mathrm{~mL}, 90 \mathrm{mmol}, 4.0$ equiv), and the mixture was stirred at room temperature for $2 \mathrm{~h}$. The reaction mixture was poured in small portions into a saturated aqueous $\mathrm{NH}_{4} \mathrm{Cl}$ solution with stirring by a pipette, and the resulting mixture was extracted with EtOAc. The extract was washed with brine, dried, and concentrated under reduced pressure. Flash chromatography on silica gel $(0 \rightarrow 10 \% \mathrm{MeOH}$ in EtOAc) afforded diol $\mathbf{S 2}(7.18 \mathrm{~g}, 93 \%)$ as a colorless solid. Mp $102-103{ }^{\circ} \mathrm{C} ;[\alpha]^{27} \mathrm{D}-22.6\left(c 1.08, \mathrm{CHCl}_{3}\right)$; IR (film) 3356, 2933, 2860, $1098 \mathrm{~cm}^{-1} ;{ }^{1} \mathrm{H}$ NMR (600 MHz, $\left.\mathrm{CDCl}_{3}\right) \delta 4.08(1 \mathrm{H}, \mathrm{dd}, J=10.2,5.0 \mathrm{~Hz})$, 3.82-3.74 (4H, m), $3.75(1 \mathrm{H}, \mathrm{t}, J=10.3 \mathrm{~Hz}), 3.54(1 \mathrm{H}, \mathrm{ddd}, J=8.1,6.8,4.6 \mathrm{~Hz}), 3.45(1 \mathrm{H}, \mathrm{ddd}, J=10.3$, 9.5, $5.0 \mathrm{~Hz}), 2.42(1 \mathrm{H}$, br s), $2.32(1 \mathrm{H}, \mathrm{br} \mathrm{s}), 1.96-1.84(5 \mathrm{H}, \mathrm{m}), 1.77(1 \mathrm{H}, \mathrm{dddd}, J=14.7,8.3,6.4,4.2$ $\mathrm{Hz}), 1.03(9 \mathrm{H}, \mathrm{s}), 0.99(9 \mathrm{H}, \mathrm{s}) ;{ }^{13} \mathrm{C} \mathrm{NMR}\left(150 \mathrm{MHz}, \mathrm{CDCl}_{3}\right) \delta 85.5,81.9,77.7,74.7,67.6,60.4,37.5$, 29.7, 29.4, 27.5, 27.1, 22.5, 20.0; HRDARTMS $m / z$ calcd for $\mathrm{C}_{17} \mathrm{H}_{35} \mathrm{O}_{5} \mathrm{Si}\left(\mathrm{MH}^{+}\right)$347.2248, found 347.2246 . 


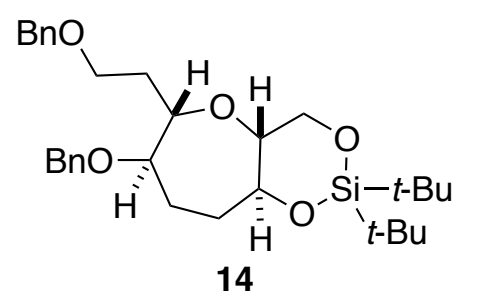

Dibenzyl ether 14. To a solution of diol S2 $(7.18 \mathrm{~g}, 20.7 \mathrm{mmol})$ and $\mathrm{BnBr}(7.40 \mathrm{~mL}, 62.2 \mathrm{mmol}, 3.0$ equiv) in THF $(100 \mathrm{~mL})$ at $0{ }^{\circ} \mathrm{C}$ was added KHMDS $(0.5 \mathrm{M}$ solution in toluene, $104 \mathrm{~mL}, 51.8 \mathrm{mmol}, 2.5$ equiv), and the reaction mixture was stirred at $0{ }^{\circ} \mathrm{C}$ for $2 \mathrm{~h}$. The reaction was quenched with saturated aqueous $\mathrm{NH}_{4} \mathrm{Cl}$ solution, and the resulting mixture was extracted with EtOAc. The extract was washed with brine, dried, and concentrated under reduced pressure. Flash chromatography on silica gel $(6 \rightarrow 10 \%$ EtOAc in $n$-hexane) afforded dibenzyl ether $\mathbf{1 4}(9.73 \mathrm{~g}, 89 \%)$ as a colorless oil. $[\alpha]^{27} \mathrm{D}-33.4(c$ 1.54, $\mathrm{CHCl}_{3}$ ); IR (film) 2932, 2858, $1096 \mathrm{~cm}^{-1} ;{ }^{1} \mathrm{H}$ NMR (600 MHz, $\left.\mathrm{CDCl}_{3}\right) \delta 7.35-7.30(8 \mathrm{H}, \mathrm{m}), 7.29-7.26$ $(2 \mathrm{H}, \mathrm{m}), 4.57$ and $4.36($ each $1 \mathrm{H}, \mathrm{d}, J=11.6 \mathrm{~Hz}), 4.51$ and $4.45($ each $1 \mathrm{H}, \mathrm{d}, J=11.9 \mathrm{~Hz}), 3.95(1 \mathrm{H}, \mathrm{dd}$, $J=10.3,5.0 \mathrm{~Hz}), 3.75(1 \mathrm{H}, \mathrm{td}, J=9.4,5.3 \mathrm{~Hz}), 3.71(1 \mathrm{H}, \mathrm{ddd}, J=9.5,5.2,3.5 \mathrm{~Hz}), 3.70(1 \mathrm{H}, \mathrm{t}, J=10.3$ Hz), 3.55-3.48 (3H, m), 3.45 (1H, ddd, $J=10.3,9.4,5.0 \mathrm{~Hz}), 2.01$ (1H, dddd, $J=14.7,6.6,4.4,2.0 \mathrm{~Hz}$ ), $1.96-1.84(3 \mathrm{H}, \mathrm{m}), 1.68-1.60(2 \mathrm{H}, \mathrm{m}), 1.02(9 \mathrm{H}, \mathrm{s}), 0.97(9 \mathrm{H}, \mathrm{s}) ;{ }^{13} \mathrm{C} \mathrm{NMR}\left(150 \mathrm{MHz}, \mathrm{CDCl}_{3}\right) \delta 138.40$, $138.39,128.4,128.3,127.7,127.59,127.56,127.5,82.1,81.3,80.4,78.2,73.1,70.7,67.4,66.9,35.1$, 29.6, 27.5, 27.1, 23.4, 22.5, 19.9; HRFABMS $m / z$ calcd for $\mathrm{C}_{31} \mathrm{H}_{46} \mathrm{O}_{5} \mathrm{SiNa}\left(\mathrm{MNa}^{+}\right) 549.3012$, found 549.3007.

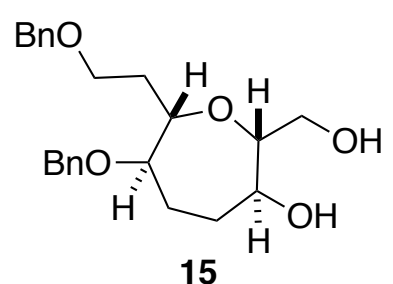

Diol 15. To a solution of silylene $14(10.6 \mathrm{~g}, 20.1 \mathrm{mmol})$ in THF $(100 \mathrm{~mL})$ was added TBAF $(1.0 \mathrm{M}$ solution in THF, $60.0 \mathrm{~mL}, 60.0 \mathrm{mmol}, 3.0$ equiv), and the reaction mixture was stirred at room temperature for $15 \mathrm{~h}$. The reaction was quenched with saturated aqueous $\mathrm{NH}_{4} \mathrm{Cl}$ solution, and THF was removed by a 
rotary evaporator. The resulting mixture was extracted with EtOAc, dried, and concentrated under reduced pressure. Flash chromatography on silica gel (40 $\rightarrow 100 \%$ EtOAc in $n$-hexane) afforded diol 15 (7.36 g, 95\%) as a colorless solid. Mp 54-56 ${ }^{\circ} \mathrm{C} ;[\alpha]^{27} \mathrm{D}-32.0\left(\mathrm{c} 0.75, \mathrm{CHCl}_{3}\right) ; \mathrm{IR}$ (film) 3400, 2928, 2867, 1071 $\mathrm{cm}^{-1} ;{ }^{1} \mathrm{H}$ NMR $\left(600 \mathrm{MHz}, \mathrm{CDCl}_{3}\right) \delta 7.35-7.26(10 \mathrm{H}, \mathrm{m}), 4.59$ and 4.37 (each $\left.1 \mathrm{H}, \mathrm{d}, J=11.5 \mathrm{~Hz}\right), 4.54$ and $4.48($ each $1 \mathrm{H}, \mathrm{d}, J=12.1 \mathrm{~Hz}), 3.75(1 \mathrm{H}, \mathrm{d}, J=10.8 \mathrm{~Hz}), 3.62(1 \mathrm{H}, \mathrm{ddd}, J=9.9,7.0,4.6 \mathrm{~Hz}), 3.58-$ $3.55(3 \mathrm{H}, \mathrm{m}), 3.53(1 \mathrm{H}, \mathrm{dd}, J=11.3,7.8 \mathrm{~Hz}), 3.38-3.34(2 \mathrm{H}, \mathrm{m}), 3.19(1 \mathrm{H}, \mathrm{br} \mathrm{s}), 2.00-1.91(2 \mathrm{H}, \mathrm{m})$, $1.90-1.79(4 \mathrm{H}, \mathrm{m}), 1.70(1 \mathrm{H}, \mathrm{dddd}, J=14.6,10.0,6.9,4.6 \mathrm{~Hz}) ;{ }^{13} \mathrm{C} \mathrm{NMR}\left(150 \mathrm{MHz}, \mathrm{CDCl}_{3}\right) \delta 138.2$, $137.9,128.38,128.36,128.0,127.72,127.68(x 2), 86.9,84.1,82.5,72.9,72.1,71.1,68.0,64.6,34.2,29.8$, 24.1; HRFABMS $m / z$ calcd for $\mathrm{C}_{23} \mathrm{H}_{30} \mathrm{O}_{5} \mathrm{Na}\left(\mathrm{MNa}^{+}\right)$409.1991, found 409.2003.

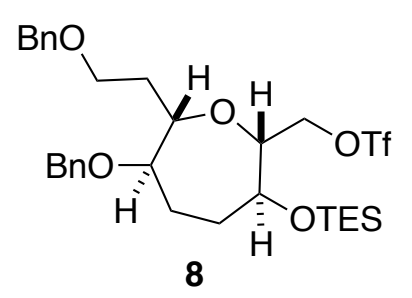

Alkyl triflate 8. To a solution of diol $15(7.50 \mathrm{~g}, 19.4 \mathrm{mmol})$ and 2,6-lutidine $(6.70 \mathrm{~mL}, 58.2 \mathrm{mmol}$, 3.0 equiv) in $\mathrm{CH}_{2} \mathrm{Cl}_{2}(98 \mathrm{~mL})$ at $-80{ }^{\circ} \mathrm{C}$ was added $\mathrm{Tf}_{2} \mathrm{O}(3.40 \mathrm{~mL}, 20.4 \mathrm{mmol}, 1.05$ equiv), and the reaction mixture was stirred at $-80^{\circ} \mathrm{C}$ for $0.5 \mathrm{~h}$. TESOTf $(4.80 \mathrm{~mL}, 21.4 \mathrm{mmol}, 1.1$ equiv) was then added, and the mixture was stirred at $-80{ }^{\circ} \mathrm{C}$ for another $0.5 \mathrm{~h}$. The reaction was quenched with saturated aqueous $\mathrm{NaHCO}_{3}$ solution, and the resulting mixture was extracted with $\mathrm{CH}_{2} \mathrm{Cl}_{2}$. The extract was washed with brine, dried, and concentrated under reduced pressure. Flash chromatography on silica gel $(6 \rightarrow 10 \%$ EtOAc in $n$-hexane) afforded alkyl triflate $8(11.9 \mathrm{~g}, 97 \%)$ as a pale yellow oil. Small amount of 2,6lutidine (ca. $1 \mu \mathrm{L}$ ) was added as a stabilizer of alkyl triflate 8 after the flash chromatography. $[\alpha]^{24} \mathrm{D}-6.0$ (c 1.01, $\left.\mathrm{CHCl}_{3}\right) ; \mathrm{IR}\left(\mathrm{CHCl}_{3}\right) 2957,2877,1414,1091 \mathrm{~cm}^{-1} ;{ }^{1} \mathrm{H} \mathrm{NMR}\left(600 \mathrm{MHz}, \mathrm{CDCl}_{3}\right) \delta 7.35-7.26(10 \mathrm{H}$, m), 4.56 and 4.39 (each 1H, d, $J=11.6 \mathrm{~Hz}), 4.55(1 \mathrm{H}, \mathrm{d}, J=10.1 \mathrm{~Hz}), 4.49$ and $4.48($ each $1 \mathrm{H}, \mathrm{d}, J=$ $11.9 \mathrm{~Hz}), 4.41(1 \mathrm{H}, \mathrm{dd}, J=10.1,5.9 \mathrm{~Hz}), 3.65(1 \mathrm{H}, \mathrm{ddd}, J=9.6,6.2,3.1 \mathrm{~Hz}), 3.60-3.56(4 \mathrm{H}, \mathrm{m}), 3.43$ $(1 \mathrm{H}, \mathrm{td}, J=5.6,3.1 \mathrm{~Hz}), 1.98-1.87(3 \mathrm{H}, \mathrm{m}), 1.77(1 \mathrm{H}, \mathrm{ddd}, J=14.0,8.6,3.0 \mathrm{~Hz}), 1.72-1.63(2 \mathrm{H}, \mathrm{m})$, 
$0.95(9 \mathrm{H}, \mathrm{t}, J=7.9 \mathrm{~Hz}), 0.59(3 \mathrm{H}, \mathrm{dq}, J=15.2,7.9 \mathrm{~Hz}), 0.58(3 \mathrm{H}, \mathrm{dq}, J=15.2,7.9 \mathrm{~Hz}) ;{ }^{13} \mathrm{C} \mathrm{NMR}(150$ $\left.\mathrm{MHz}, \mathrm{CDCl}_{3}\right) \delta 138.6,138.2,128.4,128.3,127.8,127.7,127.6,127.4,118.6\left(\mathrm{q}, J_{\mathrm{C}-\mathrm{F}}=319.5 \mathrm{~Hz}\right), 82.9$, $81.8(\mathrm{x} 2), 77.5,72.9,71.4,71.0,66.6,34.8,29.3,23.4,6.7,5.0 ;$ HRFABMS $\mathrm{m} / z$ calcd for $\mathrm{C}_{30} \mathrm{H}_{43} \mathrm{O}_{7} \mathrm{~F}_{3} \mathrm{SSiNa}\left(\mathrm{MNa}^{+}\right)$655.2349, found 655.2338.

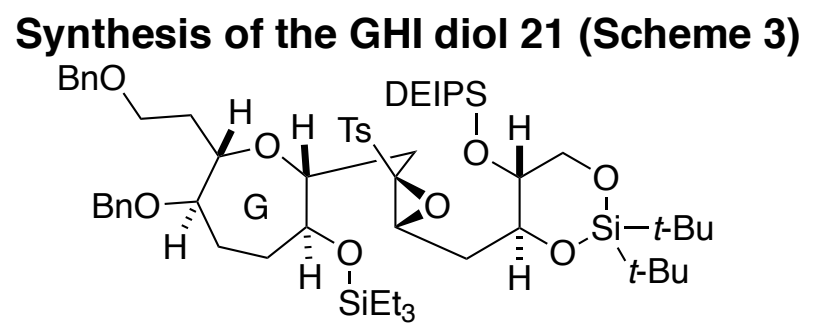

16 ( $\beta$-epoxide : $\alpha$-epoxide $=88: 12)$

Coupling product 16. To a solution of alkyl triflate $8(6.01 \mathrm{~g}, 9.50 \mathrm{mmol})$ and epoxy sulfone $\mathbf{7}^{2}(85: 15$ mixture of $\beta$ - and $\alpha$-epoxides, $8.55 \mathrm{~g}, 15.0 \mathrm{mmol}, 1.6$ equiv) in THF (75 mL) and HMPA (3.2 mL, 28.5 mmol, 3.0 equiv) at $-100{ }^{\circ} \mathrm{C}$ was added $n$-BuLi (1.63 M solution in $n$-hexane, $8.8 \mathrm{~mL}, 14.3 \mathrm{mmol}, 1.5$ equiv). The reaction mixture was stirred at $-100{ }^{\circ} \mathrm{C}$ for $0.5 \mathrm{~h}$, and the reaction was quenched with saturated aqueous $\mathrm{NH}_{4} \mathrm{Cl}$ solution. After warming to room temperature, the resulting mixture was extracted with EtOAc. The extract was washed with water and brine, dried, and concentrated under reduced pressure. Flash chromatography on silica gel $\left(5 \rightarrow 10 \% \mathrm{Et}_{2} \mathrm{O}\right.$ in $n$-hexane) afforded the coupling product $16(8.60$ g, 86\%, dr = 88:12) and starting epoxy sulfone 7 (3.68 g, 94\% recovery based on consumed 7, $\mathrm{dr}=73: 27)$.

Coupling product 16. Colorless oil; $[\alpha]^{23} \mathrm{D}-32.9\left(c\right.$ 1.02, $\left.\mathrm{CHCl}_{3}\right)$; IR $\left(\mathrm{CHCl}_{3}\right) 2958,2936,2877,2862$, $1105 \mathrm{~cm}^{-1} ;{ }^{1} \mathrm{H}$ NMR for the major isomer $\left(600 \mathrm{MHz}, \mathrm{CDCl}_{3}\right) \delta 7.77(2 \mathrm{H}, \mathrm{d}, J=8.3 \mathrm{~Hz}), 7.36-7.25(10 \mathrm{H}$, m), $7.20(2 \mathrm{H}, \mathrm{d}, J=8.3 \mathrm{~Hz}), 4.50$ and $4.46($ each $1 \mathrm{H}, \mathrm{d}, J=11.7 \mathrm{~Hz}), 4.48$ and $4.33($ each $1 \mathrm{H}, \mathrm{d}, J=11.7$ Hz), $4.02(1 \mathrm{H}, \mathrm{dd}, J=10.6,4.8 \mathrm{~Hz}), 3.81(1 \mathrm{H}, \mathrm{td}, J=9.1,2.5 \mathrm{~Hz}), 3.73(1 \mathrm{H}, \mathrm{t}, J=10.2 \mathrm{~Hz}), 3.73(1 \mathrm{H}$, $\mathrm{dd}, J=4.4,3.8 \mathrm{~Hz}), 3.63(1 \mathrm{H}, \mathrm{td}, J=8.8,5.6 \mathrm{~Hz}), 3.58(1 \mathrm{H}, \mathrm{ddd}, J=9.2,8.3,6.4 \mathrm{~Hz}), 3.54(1 \mathrm{H}, \mathrm{ddd}, J$ $=10.2,8.8,4.8 \mathrm{~Hz}), 3.32(1 \mathrm{H}, \mathrm{ddd}, J=8.0,6.0,4.2 \mathrm{~Hz}), 3.28(1 \mathrm{H}, \mathrm{td}, J=6.0,3.1 \mathrm{~Hz}), 3.26-3.22(2 \mathrm{H}$, m), $2.93(1 \mathrm{H}, \mathrm{d}, J=15.2 \mathrm{~Hz}), 2.32(3 \mathrm{H}, \mathrm{s}), 1.96(1 \mathrm{H}, \mathrm{ddd}, J=14.0,8.2,2.6 \mathrm{~Hz}), 1.84(1 \mathrm{H}, \mathrm{ddd}, J=14.0$, 9.5, 4.4 Hz), 1.82 (1H, dddd, $J=14.0,8.3,6.4,4.2 \mathrm{~Hz}), 1.76-1.56(6 \mathrm{H}, \mathrm{m}), 1.06(9 \mathrm{H}, \mathrm{s}), 0.98(9 \mathrm{H}, \mathrm{s})$, 
$0.97-0.92(22 \mathrm{H}, \mathrm{m}), 0.64-0.58(10 \mathrm{H}, \mathrm{m}) ;{ }^{13} \mathrm{C} \mathrm{NMR}$ for the major isomer $\left(150 \mathrm{MHz}, \mathrm{CDCl}_{3}\right) \delta 144.9$, $138.9,138.7,133.5,129.8,129.5,128.3,128.2,127.6,127.5,127.4,127.3,82.3,82.1,80.9,76.7,75.6$, $74.6,72.7,71.1,70.6,69.1,67.8,57.4,35.1,32.8,29.3,28.4,27.5,27.0,23.6,22.6,21.6,19.9,17.3,17.2$, 12.9, 7.1, 7.0, 6.9, 5.1, 4.0, 3.8; HRFABMS $m / z$ calcd for $\mathrm{C}_{57} \mathrm{H}_{92} \mathrm{O}_{10} \mathrm{SSi}_{3} \mathrm{Na}\left(\mathrm{MNa}^{+}\right) 1075.5617$, found 1075.5631. ${ }^{1} \mathrm{H}$ and ${ }^{13} \mathrm{C}$ NMR peaks for the minor isomer could not be fully assigned because of too weak signals. The diastereomeric ratio was determined by integration of the signals of the benzylic protons: $4.33 \mathrm{ppm}(1 \mathrm{H}, \mathrm{d}, J=11.7 \mathrm{~Hz})$ for the major isomer and $4.39 \mathrm{ppm}(1 \mathrm{H}, \mathrm{d}, J=11.7 \mathrm{~Hz})$ for the minor isomer.

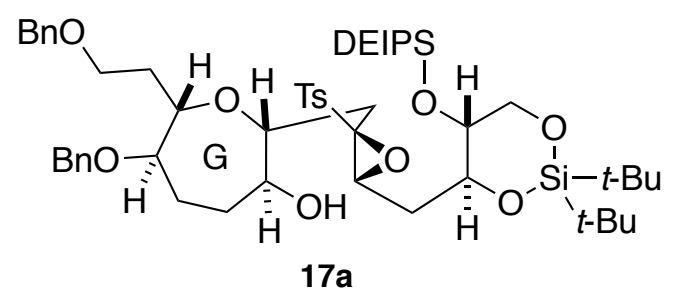

Epoxy alcohols 17a and 17b. To a solution of TES ether 16 (16.6 g, $15.8 \mathrm{mmol})$ in $\mathrm{MeOH}(158 \mathrm{~mL})$ and $\mathrm{CH}_{2} \mathrm{Cl}_{2}(158 \mathrm{~mL})$ at $0{ }^{\circ} \mathrm{C}$ was added PPTS $(793 \mathrm{mg})$, and the reaction mixture was stirred at $0{ }^{\circ} \mathrm{C}$ for $5 \mathrm{~h}$. The reaction was quenched with $\mathrm{Et}_{3} \mathrm{~N}$, and the mixture was concentrated under reduced pressure. Flash chromatography on silica gel (15\% EtOAc in $n$-hexane) afforded the major $\beta$-epoxide 17a (12.2 g, $83 \%)$ and the minor $\alpha$-epoxide $17 \mathbf{b}(1.58 \mathrm{~g}, 11 \%)$.

Major $\beta$-epoxide 17a. Colorless oil; $[\alpha]^{24}-33.7$ ( c 1.00, $\left.\mathrm{CHCl}_{3}\right)$; IR $\left(\mathrm{CHCl}_{3}\right)$ 3524, 2933, 2861, 1105 $\mathrm{cm}^{-1} ;{ }^{1} \mathrm{H}$ NMR $\left(600 \mathrm{MHz}, \mathrm{CDCl}_{3}\right) \delta 7.81(2 \mathrm{H}, \mathrm{d}, J=8.3 \mathrm{~Hz}), 7.38-7.34(8 \mathrm{H}, \mathrm{m}), 7.32-7.28(4 \mathrm{H}, \mathrm{m}), 4.57$ and 4.40 (each 1H, d, $J=11.6 \mathrm{~Hz}), 4.55$ and 4.49 (each 1H, d, $J=11.9 \mathrm{~Hz}), 4.02(1 \mathrm{H}, \mathrm{dd}, J=10.5,4.5$ $\mathrm{Hz}), 3.81(1 \mathrm{H}, \mathrm{td}, J=9.2,2.4 \mathrm{~Hz}), 3.74(1 \mathrm{H}, \mathrm{t}, J=10.3 \mathrm{~Hz}), 3.66-3.61(4 \mathrm{H}, \mathrm{m}), 3.57-3.51(2 \mathrm{H}, \mathrm{m}), 3.38$ $(1 \mathrm{H}, \mathrm{ddd}, J=6.4,5.0,3.4 \mathrm{~Hz}), 3.30(1 \mathrm{H}, \mathrm{qd}, J=8.4,5.3 \mathrm{~Hz}), 2.71(1 \mathrm{H}, \mathrm{dd}, J=15.9,3.2 \mathrm{~Hz}), 2.42(3 \mathrm{H}$, s), $2.03(1 \mathrm{H}, \mathrm{ddd}, J=13.9,7.9,2.4 \mathrm{~Hz}), 1.98-1.89(3 \mathrm{H}, \mathrm{m}), 1.85-1.78(4 \mathrm{H}, \mathrm{m}), 1.72-1.66(2 \mathrm{H}, \mathrm{m}), 1.07$ $(9 \mathrm{H}, \mathrm{s}), 0.99(9 \mathrm{H}, \mathrm{s}), 0.97-0.94(12 \mathrm{H}, \mathrm{m}), 0.89(1 \mathrm{H}, \mathrm{m}), 0.64-0.59(4 \mathrm{H}, \mathrm{m}) ;{ }^{13} \mathrm{C} \mathrm{NMR}\left(150 \mathrm{MHz}, \mathrm{CDCl}_{3}\right)$ $\delta 145.3,138.8,138.5,132.3,129.9,129.7,128.33,128.26,127.63,127.61,127.5,127.4,82.8,82.2,81.3$, 
$76.6,75.2,74.7,72.8,71.1,70.9,68.9,67.6,58.5,35.1,32.9,29.64,29.62,27.5,27.0,23.9,22.6,21.7$ 19.8, 17.3, 17.2, 12.8, 7.1, 7.0, 4.0, 3.8; HRDARTMS $m / z$ calcd for $\mathrm{C}_{51} \mathrm{H}_{79} \mathrm{O}_{10} \mathrm{SSi}\left(\mathrm{MH}^{+}\right)$939.4927, found 939.4922.

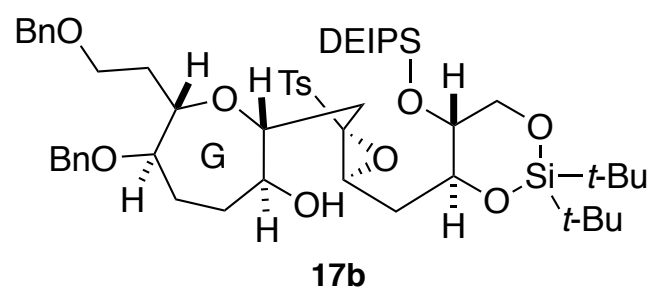

Minor $\alpha$-epoxide 17b. Colorless oil; $[\alpha]^{25} \mathrm{D}-70.4\left(c\right.$ 1.69, $\left.\mathrm{CHCl}_{3}\right)$; IR $\left(\mathrm{CHCl}_{3}\right)$ 3520, 2933, 2861, 1107 $\mathrm{cm}^{-1} ;{ }^{1} \mathrm{H}$ NMR $\left(600 \mathrm{MHz}, \mathrm{CDCl}_{3}\right) \delta 7.83(2 \mathrm{H}, \mathrm{d}, J=8.3 \mathrm{~Hz}), 7.41-7.28(12 \mathrm{H}, \mathrm{m}), 4.60$ and 4.57 (each $1 \mathrm{H}, \mathrm{d}, J=11.7 \mathrm{~Hz}), 4.59$ and $4.42($ each $1 \mathrm{H}, \mathrm{d}, J=11.7 \mathrm{~Hz}), 4.01(1 \mathrm{H}, \mathrm{dd}, J=10.5,4.6 \mathrm{~Hz}), 3.94(1 \mathrm{H}$, ddd, $J=10.2,8.8,2.9 \mathrm{~Hz}), 3.89(1 \mathrm{H}, \mathrm{ddd}, J=10.6,9.0,1.8 \mathrm{~Hz}), 3.88(1 \mathrm{H}, \mathrm{dd}, J=9.2,3.3 \mathrm{~Hz}), 3.75(1 \mathrm{H}$, t, $J=10.3 \mathrm{~Hz}), 3.69-3.63(3 \mathrm{H}, \mathrm{m}), 3.51(1 \mathrm{H}, \mathrm{ddd}, J=9.9,9.0,4.8 \mathrm{~Hz}), 3.40(1 \mathrm{H}, \mathrm{td}, J=7.3,3.9 \mathrm{~Hz})$, $3.27(1 \mathrm{H}, \mathrm{tdd}, J=9.5,6.2,3.9 \mathrm{~Hz}), 2.68(1 \mathrm{H}, \mathrm{dd}, J=15.6,2.8 \mathrm{~Hz}), 2.44(3 \mathrm{H}, \mathrm{s}), 2.04-1.92(4 \mathrm{H}, \mathrm{m}), 1.86-$ $1.79(3 \mathrm{H}, \mathrm{m}), 1.74-1.68(2 \mathrm{H}, \mathrm{m}), 1.57(1 \mathrm{H}, \mathrm{d}, J=6.6 \mathrm{~Hz}), 1.03(9 \mathrm{H}, \mathrm{s}), 0.97-0.94(12 \mathrm{H}, \mathrm{m}), 0.96(9 \mathrm{H}$, s), $0.89(1 \mathrm{H}, \mathrm{m}), 0.64-0.57(4 \mathrm{H}, \mathrm{m}) ;{ }^{13} \mathrm{C} \mathrm{NMR}\left(150 \mathrm{MHz}, \mathrm{CDCl}_{3}\right) \delta 145.2,138.8,138.5,132.0,130.0$, $129.5,128.32,128.25,127.8,127.6,127.5,127.3,82.9,81.8,81.3,76.3,75.9,74.0,73.0,71.7,70.9,69.2$, 67.6, 58.8, 35.3, 34.0, 32.3, 29.6, 27.5, 27.0, 24.2, 22.6, 21.7, 19.8, 17.25, 17.21, 12.8, 7.1, 6.9, 3.9, 3.7; HRDARTMS $m / z$ calcd for $\mathrm{C}_{51} \mathrm{H}_{79} \mathrm{O}_{10} \mathrm{SSi}\left(\mathrm{MH}^{+}\right)$939.4927, found 939.4924.

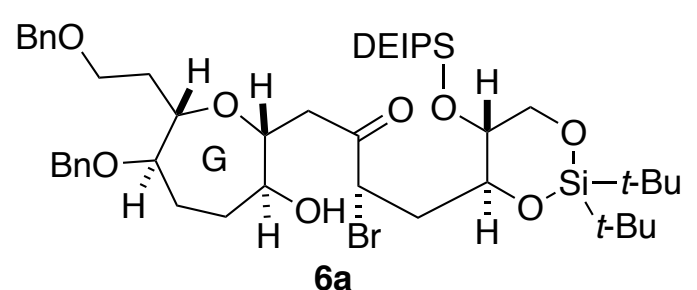

-B-Bromide 6a. To a solution of the major epoxy sulfone $\mathbf{1 7 a}(12.2 \mathrm{~g}, 13.1 \mathrm{mmol})$ and $\mathrm{LiBr}(2.26 \mathrm{~g}$, $26.1 \mathrm{mmol}, 2.0$ equiv) in $\mathrm{CH}_{2} \mathrm{Cl}_{2}(130 \mathrm{~mL})$ at $-15^{\circ} \mathrm{C}$ was added $\mathrm{MgBr}_{2} \cdot \mathrm{OEt}_{2}(6.74 \mathrm{~g}, 26.1 \mathrm{mmol}, 2.0$ 
equiv), and the reaction mixture was stirred at $-15{ }^{\circ} \mathrm{C}$ for $40 \mathrm{~min}$. The reaction was quenched with saturated aqueous $\mathrm{NaHCO}_{3}$ solution and the resulting mixture was filtered through a Celite pad. The filtrate was extracted with $\mathrm{CH}_{2} \mathrm{Cl}_{2}$ and the extract was washed with brine, dried, and concentrated under reduced pressure. Flash chromatography on silica gel $(16 \rightarrow 20 \%$ EtOAc in $n$-hexane) afforded $\alpha$-bromide 6a $(10.7 \mathrm{~g}, 95 \%)$ as a pale yellow oil. $\alpha$-Bromide $\mathbf{6 a}$ was a mixture of tautomers of a bromoketone form 6a and its hemiacetal forms $6 \mathbf{a}^{\prime}$ and $\mathbf{6 a}^{\prime \prime}$ in $\mathrm{CDCl}_{3}\left(\mathbf{6 a}: \mathbf{6} \mathbf{a}^{\prime}: \mathbf{6} \mathbf{a}^{\prime \prime}=70: 15: 15\right)$. $[\alpha]^{24} \mathrm{D}-82.2\left(c 1.05, \mathrm{CHCl}_{3}\right)$; IR $\left(\mathrm{CHCl}_{3}\right)$ 3436, 2933, 2861, 1716, $1105 \mathrm{~cm}^{-1} ;{ }^{1} \mathrm{H} \mathrm{NMR}$ for bromoketone form $\mathbf{6 a}\left(600 \mathrm{MHz}, \mathrm{CDCl}_{3}\right) \delta$ 7.35-7.24 (10H, m), $4.64(1 \mathrm{H}, \mathrm{dd}, J=10.6,3.5 \mathrm{~Hz}), 4.55(1 \mathrm{H}, \mathrm{d}, J=11.6 \mathrm{~Hz}), 4.50(1 \mathrm{H}, \mathrm{d}, J=11.7 \mathrm{~Hz})$, $4.45(1 \mathrm{H}, \mathrm{d}, J=11.7 \mathrm{~Hz}), 4.40(1 \mathrm{H}, \mathrm{d}, J=11.6 \mathrm{~Hz}), 4.09-3.99(2 \mathrm{H}, \mathrm{m}), 3.81(1 \mathrm{H}, \mathrm{ddd}, J=9.4,6.6,5.1$ Hz), $3.79(1 \mathrm{H}, \mathrm{t}, J=10.3 \mathrm{~Hz}), 3.69(1 \mathrm{H}, \mathrm{ddd}, J=9.6,6.3,3.0 \mathrm{~Hz}), 3.63-3.49(3 \mathrm{H}, \mathrm{m}), 3.44(1 \mathrm{H}, \mathrm{m}), 3.39$ $(1 \mathrm{H}, \mathrm{td}, J=5.7,3.2 \mathrm{~Hz}), 3.03(1 \mathrm{H}, \mathrm{dd}, J=16.9,5.1 \mathrm{~Hz}), 3.02(1 \mathrm{H}, \mathrm{dd}, J=16.9,6.6 \mathrm{~Hz}), 2.46(1 \mathrm{H}, \mathrm{ddd}$, $J=14.5,10.6,2.0 \mathrm{~Hz}), 1.96-1.82(5 \mathrm{H}, \mathrm{m}), 1.75-1.70(1 \mathrm{H}, \mathrm{m}), 1.67-1.55(1 \mathrm{H}, \mathrm{m}), 1.63(1 \mathrm{H}, \mathrm{d}, J=6.2$ $\mathrm{Hz}), 1.06(9 \mathrm{H}, \mathrm{s}), 0.99-0.95(12 \mathrm{H}, \mathrm{m}), 0.94(9 \mathrm{H}, \mathrm{s}), 0.90(1 \mathrm{H}, \mathrm{m}), 0.65-0.59(4 \mathrm{H}, \mathrm{m}) ;{ }^{13} \mathrm{C}$ NMR for bromoketone form $\mathbf{6 a}\left(150 \mathrm{MHz}, \mathrm{CDCl}_{3}\right) \delta 201.9,138.7,138.4,128.3,128.2,127.8,127.61,127.57$, $127.4,82.1,81.9,81.6,76.0,74.4,73.0,71.4,70.9,69.2,67.4,50.8,44.2,37.9,35.2,29.7,27.5,27.0$, 23.9, 22.7, 19.8, 17.18, 17.16, 12.8, 7.0, 6.9, 3.9, 3.7; ${ }^{1} \mathrm{H}$ NMR for hemiacetal forms $\mathbf{6} \mathbf{a}^{\prime}$ and $\mathbf{6} \mathbf{a}^{\prime \prime}(600$ $\left.\mathrm{MHz}, \mathrm{CDCl}_{3}\right) \delta 7.35-7.24(10 \mathrm{H}, \mathrm{m}), 4.56(1 \mathrm{H}, \mathrm{d}, J=11.9 \mathrm{~Hz}), 4.500(0.5 \mathrm{H}, \mathrm{d}, J=11.9 \mathrm{~Hz}), 4.496(0.5 \mathrm{H}$, $\mathrm{d}, J=11.9 \mathrm{~Hz}), 4.47(0.5 \mathrm{H}, \mathrm{d}, J=11.9 \mathrm{~Hz}), 4.46(0.5 \mathrm{H}, \mathrm{d}, J=11.9 \mathrm{~Hz}), 4.43(0.5 \mathrm{H}, \mathrm{d}, J=11.9 \mathrm{~Hz}), 4.41$ $(0.5 \mathrm{H}, \mathrm{d}, J=11.9 \mathrm{~Hz}), 4.41-4.36(1.5 \mathrm{H}, \mathrm{m}), 4.31(0.5 \mathrm{H}, \mathrm{dd}, J=10.0,3.4 \mathrm{~Hz}), 4.09-3.99(2 \mathrm{H}, \mathrm{m}), 3.96$ $(0.5 \mathrm{H}, \mathrm{td}, J=6.7,1.6 \mathrm{~Hz}), 3.87(0.5 \mathrm{H}, \mathrm{ddd}, J=11.1,8.8,5.0 \mathrm{~Hz}), 3.79(1 \mathrm{H}, \mathrm{t}, J=10.3 \mathrm{~Hz}), 3.63-3.49$ $(5 \mathrm{H}, \mathrm{m}), 3.47(0.5 \mathrm{H}, \mathrm{br} \mathrm{s}), 2.77(0.5 \mathrm{H}$, br s) $, 2.50(0.5 \mathrm{H}, \mathrm{dd}, J=13.7,9.3 \mathrm{~Hz}), 2.40(0.5 \mathrm{H}, \mathrm{ddd}, J=14.5$, 10.0, 1.5 Hz), $2.29(0.5 \mathrm{H}, \mathrm{ddd}, J=13.8,11.6,1.9 \mathrm{~Hz}), 2.25(0.5 \mathrm{H}, \mathrm{ddd}, J=12.3,10.3,1.0 \mathrm{~Hz}), 2.22(0.5 \mathrm{H}$, $\mathrm{dd}, J=12.3,7.5 \mathrm{~Hz}), 2.07-1.82(5 \mathrm{H}, \mathrm{m}), 1.75-1.70(1 \mathrm{H}, \mathrm{m}), 1.67-1.55(1.5 \mathrm{H}, \mathrm{m}), 1.054(4.5 \mathrm{H}, \mathrm{s}), 1.049$ $(4.5 \mathrm{H}, \mathrm{s}), 0.99-0.95(21 \mathrm{H}, \mathrm{m}), 0.90(1 \mathrm{H}, \mathrm{m}), 0.65-0.59(4 \mathrm{H}, \mathrm{m}) ;{ }^{13} \mathrm{C}$ NMR for hemiacetal forms $\mathbf{6} \mathbf{a}^{\prime}$ and 6a" $\left(150 \mathrm{MHz}, \mathrm{CDCl}_{3}\right) \delta 138.5,138.4,138.3,138.2,128.4,128.34$ (x2), 128.31, 127.6, 127.54, 127.52 
(x2), 127.47, 127.45, 127.41(x2), 104.8, 104.3, 84.9, 81.6, 80.9, 80.8, 79.6, 79.5, 79.2, 78.1, 76.7, 76.1, $73.0(x 2), 71.6,71.4,70.4(x 2), 69.1,69.0,67.10,67.05,59.1,58.3,42.0,40.4,39.7,38.8,34.9(x 2), 27.4$ (x2), $27.1(x 2), 25.0,23.6,22.65,22.61,22.1,21.7,19.8(x 2), 17.18(x 2), 17.16(x 2), 12.9,12.8,7.0(x 2)$, 6.9 (x2), 3.9 (x2), 3.7 (x2). HRDARTMS m/z calcd for $\mathrm{C}_{44} \mathrm{H}_{75} \mathrm{O}_{8} \mathrm{NBrSi}_{2}\left(\mathrm{M}+\mathrm{NH}_{4}{ }^{+}\right)$880.4209, found 880.4203.

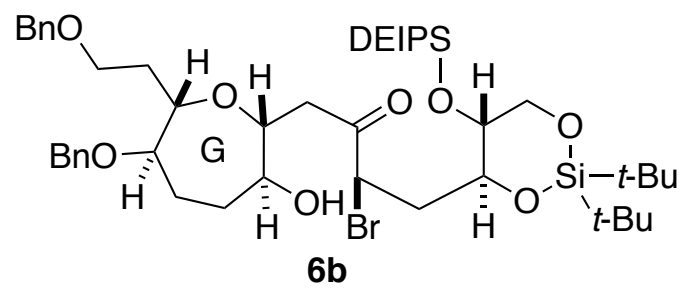

$\boldsymbol{\beta}$-Bromide $\mathbf{6 b}$. To a solution of minor epoxy sulfone $\mathbf{1 7 b}(1.56 \mathrm{~g}, 1.67 \mathrm{mmol})$ and $\mathrm{LiBr}(289 \mathrm{mg}, 3.33$ mmol, 2.0 equiv) in $\mathrm{CH}_{2} \mathrm{Cl}_{2}(17 \mathrm{~mL})$ at $-15^{\circ} \mathrm{C}$ was added $\mathrm{MgBr}_{2} \cdot \mathrm{OEt}_{2}(2.15 \mathrm{~g}, 8.33 \mathrm{mmol}, 5.0$ equiv), and the reaction mixture was stirred at $0{ }^{\circ} \mathrm{C}$ for $4 \mathrm{~h}$. The reaction was quenched with saturated aqueous $\mathrm{NaHCO}_{3}$ solution and the resulting mixture was filtered through a Celite pad. The filtrate was extracted with $\mathrm{CH}_{2} \mathrm{Cl}_{2}$ and the extract was washed with brine, dried, and concentrated under reduced pressure. Flash chromatography on silica gel $(10 \rightarrow 16 \%$ EtOAc in $n$-hexane) afforded $\beta$-bromide $6 \mathbf{b}(1.28 \mathrm{~g}, 89 \%)$ and $\alpha$-bromide $6 \mathbf{a}(88 \mathrm{mg}, 6 \%)$. When the reaction time was extended to $20 \mathrm{~h}, \beta$-bromide $\mathbf{6 b}(10 \%)$ and $\alpha$ bromide 6a $(65 \%)$ were obtained as shown in Scheme 3. $\beta$-Bromide $6 \mathbf{b}$ was obtained as a mixture of tautomers of bromoketone form $\mathbf{6 b}$ and its hemiacetal forms $6 \mathbf{b}^{\prime}$ and $\mathbf{6} \mathbf{b}^{\prime \prime}\left(\mathbf{6 b}: \mathbf{6} \mathbf{b}^{\prime}: \mathbf{6} \mathbf{b}^{\prime \prime}=66: 28: 8\right)$. Pale yellow oil; $[\alpha]^{26} \mathrm{D}-39.9\left(c\right.$ 1.55, $\left.\mathrm{CHCl}_{3}\right)$; IR $\left(\mathrm{CHCl}_{3}\right)$ 3357, 2933, 2861, 1719, $1103 \mathrm{~cm}^{-1}$; ${ }^{1} \mathrm{H}$ NMR for bromoketone form $\mathbf{6 b}\left(600 \mathrm{MHz}, \mathrm{CDCl}_{3}\right) \delta 7.35-7.24(10 \mathrm{H}, \mathrm{m}), 4.60(1 \mathrm{H}, \mathrm{dd}, J=10.3,4.0 \mathrm{~Hz}), 4.56$ and $4.40($ each $1 \mathrm{H}, \mathrm{d}, J=11.7 \mathrm{~Hz}), 4.52$ and $4.46($ each $1 \mathrm{H}, \mathrm{d}, J=11.7 \mathrm{~Hz}), 3.98(1 \mathrm{H}, \mathrm{dd}, J=10.6,4.6 \mathrm{~Hz})$, $3.81(1 \mathrm{H}, \mathrm{m}), 3.78(1 \mathrm{H}, \mathrm{td}, J=9.5,2.6 \mathrm{~Hz}), 3.70(1 \mathrm{H}, \mathrm{t}, J=10.3 \mathrm{~Hz}), 3.67(1 \mathrm{H}, \mathrm{ddd}, J=9.3,6.6,3.3 \mathrm{~Hz})$, 3.55-3.50 (3H, m), $3.47(1 \mathrm{H}, \mathrm{m}), 3.39(1 \mathrm{H}, \mathrm{td}, J=5.9,3.2 \mathrm{~Hz}), 3.14(1 \mathrm{H}, \mathrm{dd}, J=17.2,3.9 \mathrm{~Hz}), 2.93(1 \mathrm{H}$, dd, $J=17.2,8.0 \mathrm{~Hz}), 2.73(1 \mathrm{H}, \mathrm{ddd}, J=13.8,10.5,2.8 \mathrm{~Hz}), 2.09(1 \mathrm{H}, \mathrm{ddd}, J=13.8,9.8,4.1 \mathrm{~Hz}), 1.98-$ $1.90(2 \mathrm{H}, \mathrm{m}), 1.90-1.80(2 \mathrm{H}, \mathrm{m}), 1.75(1 \mathrm{H}, \mathrm{m}), 1.72-1.67(2 \mathrm{H}, \mathrm{m}), 1.00(9 \mathrm{H}, \mathrm{s}), 0.99(9 \mathrm{H}, \mathrm{s}), 1.00-0.94$ 
$(12 \mathrm{H}, \mathrm{m}), 0.90(1 \mathrm{H}, \mathrm{m}), 0.66-0.60(4 \mathrm{H}, \mathrm{m}) ;{ }^{13} \mathrm{C} \mathrm{NMR}$ for bromoketone form $\mathbf{6 b}\left(150 \mathrm{MHz}, \mathrm{CDCl}_{3}\right) \delta$ $202.0,138.7,138.4,128.3,128.2,127.7,127.6,127.5,127.4,82.0,81.8,81.3,76.4,74.5,72.9,71.5,70.9$ 69.0, 67.4, 50.0, 43.8, 39.8, 35.1, 29.6, 27.4, 27.0, 23.9, 22.5, 19.9, 17.21, 17.18, 12.9, 7.0, 6.9, 4.0, 3.8; ${ }^{1} \mathrm{H}$ NMR for the major hemiacetal form $\mathbf{6} \mathbf{b}^{\prime}\left(600 \mathrm{MHz}, \mathrm{CDCl}_{3}\right) \delta 7.35-7.24(10 \mathrm{H}, \mathrm{m}), 5.51(1 \mathrm{H}$, br s), 4.55 and 4.42 (each $1 \mathrm{H}, \mathrm{d}, J=11.9 \mathrm{~Hz}), 4.49$ and 4.45 (each $1 \mathrm{H}, \mathrm{d}, J=11.9 \mathrm{~Hz}), 4.26(1 \mathrm{H}, \mathrm{td}, J=9.0$, $1.8 \mathrm{~Hz}), 4.24(1 \mathrm{H}, \mathrm{dd}, J=6.4,2.7 \mathrm{~Hz}), 4.00(1 \mathrm{H}, \mathrm{dd}, J=10.6,4.6 \mathrm{~Hz}), 3.95(1 \mathrm{H}, \mathrm{td}, J=6.8,1.3 \mathrm{~Hz}), 3.91$ $(1 \mathrm{H}, \mathrm{q}, J=9.0 \mathrm{~Hz}), 3.83(1 \mathrm{H}, \mathrm{m}), 3.82(1 \mathrm{H}, \mathrm{m}), 3.59(1 \mathrm{H}, \mathrm{m}), 3.58(1 \mathrm{H}, \mathrm{m}), 3.55-3.50(2 \mathrm{H}, \mathrm{m}), 2.62(1 \mathrm{H}$, $\mathrm{dd}, J=13.5,8.7 \mathrm{~Hz}), 2.38(1 \mathrm{H}, \mathrm{ddd}, J=15.0,9.0,2.8 \mathrm{~Hz}), 2.33(1 \mathrm{H}$, ddd, $J=15.0,6.2,2.0 \mathrm{~Hz}), 2.03$ $(1 \mathrm{H}, \mathrm{m}), 1.97(1 \mathrm{H}, \mathrm{dd}, J=13.4,9.4 \mathrm{~Hz}), 1.96-1.93(2 \mathrm{H}, \mathrm{m}), 1.81(1 \mathrm{H}, \mathrm{m}), 1.66-1.62(2 \mathrm{H}, \mathrm{m}), 1.06(9 \mathrm{H}$, s), $1.00(9 \mathrm{H}, \mathrm{s}), 1.00-0.94(12 \mathrm{H}, \mathrm{m}), 0.90(1 \mathrm{H}, \mathrm{m}), 0.66-0.60(4 \mathrm{H}, \mathrm{m}) ;{ }^{13} \mathrm{C}$ NMR for the major hemiacetal form $\mathbf{6 b}^{\prime}\left(150 \mathrm{MHz}, \mathrm{CDCl}_{3}\right) \delta 138.5,138.4,128.34,128.32,127.6,127.53,127.50,127.4,103.9,81.8$, $81.3,79.3,79.1,77.2,73.0,70.9,70.4,68.5,67.1,58.4,43.9,36.3,35.1,27.3,26.8,24.0,22.5,22.0,20.0$, 17.21, 17.18, 12.9, 7.0, 6.9, 4.1, 3.8; HRDARTMS $m / z$ calcd for $\mathrm{C}_{44} \mathrm{H}_{75} \mathrm{O}_{8} \mathrm{NBrSi}_{2}\left(\mathrm{M}+\mathrm{NH}_{4}^{+}\right)$880.4209, found $880.4195 .{ }^{1} \mathrm{H}$ NMR and ${ }^{13} \mathrm{C}$ NMR peaks for the minor hemiacetal $6 \mathbf{b}^{\prime \prime}$ could not be fully assigned because of too weak signals. The diastereomeric ratio was determined by integration of the signals of the methylene protons: $2.73 \mathrm{ppm}(1 \mathrm{H}$, ddd, $J=13.8,10.5,2.8 \mathrm{~Hz})$ for the bromoketone $\mathbf{6 b}$, and $2.33(1 \mathrm{H}$, $\mathrm{ddd}, J=15.0,6.2,2.0 \mathrm{~Hz})$ for the major hemiacetal $\mathbf{6} \mathbf{b}^{\prime}$, and $2.26(1 \mathrm{H}, \mathrm{d}, J=12.0,6.9 \mathrm{~Hz})$ for the minor hemiacetal $6 \mathbf{b}^{\prime \prime}$.
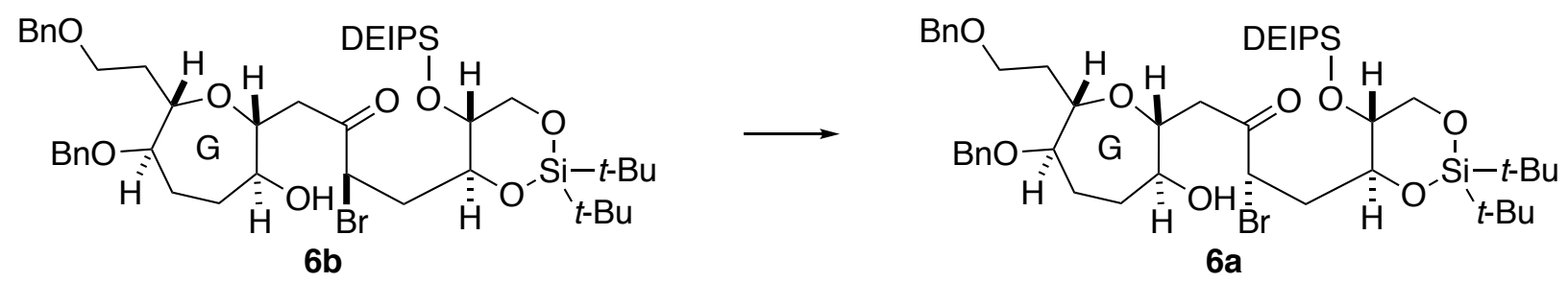

Epimerization experiment from $\beta$-bromide $6 \mathrm{~b}$ to $\alpha$-bromide 6 a (Scheme 4). To a solution of $\beta$ bromide $6 \mathbf{b}(641 \mathrm{mg}, 0.743 \mathrm{mmol})$ and $\mathrm{LiBr}\left(129 \mathrm{mg}, 1.49 \mathrm{mmol}, 2.0\right.$ equiv) in $\mathrm{CH}_{2} \mathrm{Cl}_{2}(7.5 \mathrm{~mL})$ at $15^{\circ} \mathrm{C}$ was added $\mathrm{MgBr}_{2} \cdot \mathrm{OEt}_{2}\left(959 \mathrm{~g}, 3.72 \mathrm{mmol}, 5.0\right.$ equiv), and the reaction mixture was stirred at $0{ }^{\circ} \mathrm{C}$ 
for $95 \mathrm{~h}$. The reaction was quenched with saturated aqueous $\mathrm{NaHCO}_{3}$ solution and the resulting mixture was extracted with $\mathrm{CH}_{2} \mathrm{Cl}_{2}$. The extract was washed with brine, dried, and concentrated under reduced pressure. Flash chromatography on silica gel $(0 \rightarrow 5 \%$ EtOAc in benzene) afforded $\alpha$-bromide $6 \mathbf{a}$ (397 $\mathrm{mg}, 62 \%)$ and $\beta$-bromide $\mathbf{6 b}(80 \mathrm{mg}, 12 \%)$.

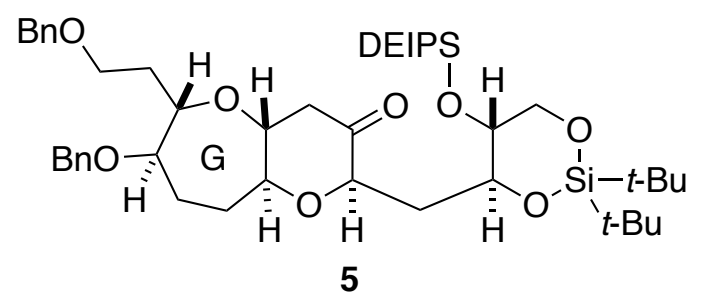

Six-membered ketone 5. To a solution of $\alpha$-bromide 6a (5.30 g, $6.14 \mathrm{mmol})$ and 18-crown-6 (1.64 g, $6.19 \mathrm{mmol}, 1.0$ equiv) in THF $(620 \mathrm{~mL})$ at $0{ }^{\circ} \mathrm{C}$ was added $1 \mathrm{M}$ aqueous $\mathrm{NaOH}$ solution $(18 \mathrm{~mL}, 0.18$ mmol, 2.9 equiv). The mixture was stirred at $0{ }^{\circ} \mathrm{C}$ for $4 \mathrm{~h}$, and the reaction was quenched with saturated aqueous $\mathrm{NH}_{4} \mathrm{Cl}$ solution. THF was removed by concentration under reduced pressure, and the resulting mixture was extracted with EtOAc. The extract was washed with brine, dried, and concentrated under reduced pressure. Flash chromatography on silica gel $(18 \rightarrow 20 \%$ EtOAc in $n$-hexane) afforded the sixmembered ketone $5(3.89 \mathrm{~g}, 81 \%)$ as a colorless oil. $[\alpha]^{26}{ }_{\mathrm{D}}-43.9\left(c\right.$ 2.17, $\left.\mathrm{CHCl}_{3}\right)$; IR $\left(\mathrm{CHCl}_{3}\right) 2934,2859$, $1725,1091 \mathrm{~cm}^{-1} ;{ }^{1} \mathrm{H} \mathrm{NMR}\left(600 \mathrm{MHz}, \mathrm{CDCl}_{3}\right) \delta 7.36-7.26(10 \mathrm{H}, \mathrm{m}), 4.58$ and 4.39 (each $1 \mathrm{H}, \mathrm{d}, J=11.7$ $\mathrm{Hz}), 4.50$ and $4.44($ each $1 \mathrm{H}, \mathrm{d}, J=11.9 \mathrm{~Hz}), 4.07(1 \mathrm{H}, \mathrm{td}, J=8.8,3.6 \mathrm{~Hz}), 4.00(1 \mathrm{H}, \mathrm{dd}, J=6.2,4.0 \mathrm{~Hz})$, $3.98(1 \mathrm{H}, \mathrm{dd}, J=10.4,4.5 \mathrm{~Hz}), 3.71(1 \mathrm{H}, \mathrm{t}, J=10.3 \mathrm{~Hz}), 3.71-3.66(2 \mathrm{H}, \mathrm{m}), 3.61(1 \mathrm{H}, \mathrm{ddd}, J=9.9,8.9$, $4.5 \mathrm{~Hz}), 3.53-3.48(3 \mathrm{H}, \mathrm{m}), 3.34(1 \mathrm{H}, \mathrm{td}, J=9.7,4.8 \mathrm{~Hz}), 2.82(1 \mathrm{H}, \mathrm{dd}, J=16.9,6.2 \mathrm{~Hz}), 2.34(1 \mathrm{H}, \mathrm{td}, J$ $=10.1,3.8 \mathrm{~Hz}), 2.33(1 \mathrm{H}, \mathrm{dd}, J=16.9,10.5 \mathrm{~Hz}), 2.07(1 \mathrm{H}, \mathrm{dddd}, J=14.5,7.6,4.2,1.3 \mathrm{~Hz}), 2.02-1.92$ $(3 \mathrm{H}, \mathrm{m}), 1.90(1 \mathrm{H}, \mathrm{dtd}, J=14.5,7.5,3.5 \mathrm{~Hz}), 1.73(1 \mathrm{H}, \mathrm{ddt}, J=14.6,12.3,2.0 \mathrm{~Hz}), 1.66(1 \mathrm{H}, \mathrm{dddd}, J=$ 14.0, 9.7, 6.1, 5.1 Hz), 0.98-0.95 (12H, m), 0.98 (9H, s), $0.96(9 \mathrm{H}, \mathrm{s}), 0.90(1 \mathrm{H}, \mathrm{m}), 0.67-0.57$ (4H, m); ${ }^{13} \mathrm{C}$ NMR (150 MHz, $\left.\mathrm{CDCl}_{3}\right) \delta 207.1,138.4,138.3,128.4(\mathrm{x} 2), 127.7,127.64,127.60$ (x2), 81.7, 81.1, $80.9,79.5,79.1,75.1,73.0,71.6,70.8,69.3,67.0,45.7,35.1,34.4,27.5,26.9,26.2,23.6,22.6,19.9$, 
17.25, 17.22, 12.9, 7.04, 6.97, 4.0, 3.8; HRDARTMS $m / z$ calcd for $\mathrm{C}_{44} \mathrm{H}_{71} \mathrm{O}_{8} \mathrm{Si}_{2}\left(\mathrm{MH}^{+}\right)$783.4682, found 783.4677.

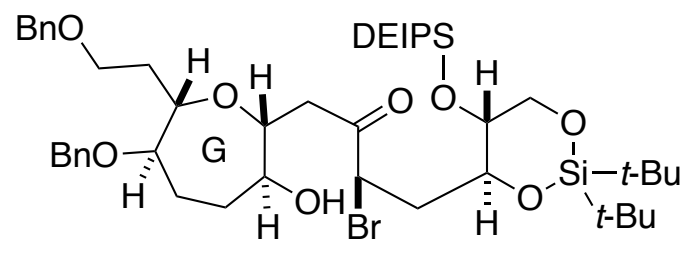

$6 \mathbf{b}$
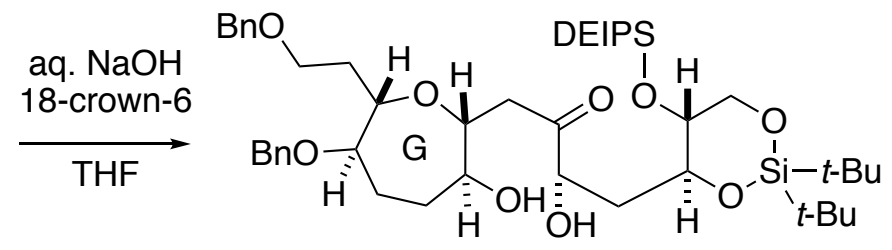

S3

Attempted cyclization of $\boldsymbol{\beta}$-bromide $\mathbf{6 b}$. To a solution of $\beta$-bromide $\mathbf{6 b}(20.0 \mathrm{mg}, 0.0232 \mathrm{mmol})$ and 18-crown-6 (6.1 mg, $0.023 \mathrm{mmol}, 1.0$ equiv) in THF $(2.3 \mathrm{~mL})$ at $0{ }^{\circ} \mathrm{C}$ was added $1 \mathrm{M}$ aqueous $\mathrm{NaOH}$ solution $\left(70 \mu \mathrm{L}, 0.070 \mathrm{mmol}, 3.0\right.$ equiv). The mixture was stirred at $0{ }^{\circ} \mathrm{C}$ for $1 \mathrm{~h}$, and the reaction was quenched with saturated aqueous $\mathrm{NH}_{4} \mathrm{Cl}$ solution. The resulting mixture was extracted with EtOAc, and the extract was washed with brine, dried, and concentrated under reduced pressure. Flash chromatography on silica gel (5\% EtOAc in benzene) afforded the $\alpha$-hydroxy ketone $\mathbf{S 3}(10.1 \mathrm{mg}, 54 \%)$ as a colorless oil. $\alpha$-hydroxy ketone $\mathbf{S 3}$ was obtained as a mixture of tautomers of hydroxy ketone form $\mathbf{S 3}$ and its hemiacetal forms $\mathbf{S 3}^{\prime}$ and $\mathbf{S 3}^{\prime \prime}\left(\mathbf{S 3 : S 3} \mathbf{S S 3}^{\prime \prime}=67: 19: 14\right) .[\alpha]^{22} \mathrm{D}-29.3\left(c\right.$ 0.75, $\left.\mathrm{CHCl}_{3}\right)$; IR (film) 3455, 2956, 2933, 2861, 1714, 1472, $1096 \mathrm{~cm}^{-1} ;{ }^{1} \mathrm{H}$ NMR for hydroxy ketone form $\mathbf{S 3}\left(600 \mathrm{MHz}, \mathrm{CDCl}_{3}\right) \delta$ 7.35-7.31 (8H, m), 7.29-7.25 (2H, m), 4.56 and 4.40 (each 1H, d, $J=11.6 \mathrm{~Hz}), 4.49$ and 4.47 (each 1H, d, $J=11.9 \mathrm{~Hz}), 4.43(1 \mathrm{H}, \mathrm{ddd}, J=9.3,5.9,2.8 \mathrm{~Hz}), 4.09(1 \mathrm{H}, \mathrm{ddd}, J=10.2,8.9,2.1 \mathrm{~Hz}), 4.03(1 \mathrm{H}, \mathrm{dd}$, $J=10.4,4.5 \mathrm{~Hz}), 3.86(1 \mathrm{H}, \mathrm{td}, J=8.8,3.8 \mathrm{~Hz}), 3.77(1 \mathrm{H}, \mathrm{t}, J=10.3 \mathrm{~Hz}), 3.66(1 \mathrm{H}, \mathrm{d}, J=5.9 \mathrm{~Hz}, \mathrm{OH})$, $3.65(1 \mathrm{H}, \mathrm{ddd}, J=9.7,6.2,3.5 \mathrm{~Hz}), 3.55(1 \mathrm{H}, \mathrm{ddd}, J=10.1,8.8,4.6 \mathrm{~Hz}), 3.46-3.43(3 \mathrm{H}, \mathrm{m}), 3.40(1 \mathrm{H}$, ddd, $J=6.2,5.1,3.1 \mathrm{~Hz}), 2.93(1 \mathrm{H}, \mathrm{dd}, J=16.3,3.9 \mathrm{~Hz}), 2.68(1 \mathrm{H}, \mathrm{dd}, J=16.1,8.8 \mathrm{~Hz}) .1 .98(1 \mathrm{H}$, ddd, $J=13.7,9.3,2.0 \mathrm{~Hz}), 1.96(1 \mathrm{H}, \mathrm{m}), 1.92(1 \mathrm{H}, \mathrm{dtd}, J=14.0,7.3,3.5 \mathrm{~Hz}), 1.91-1.85(2 \mathrm{H}, \mathrm{m}), 1.75(1 \mathrm{H}$, ddd, $J=13.7,10.3,2.8 \mathrm{~Hz}), 1.72(1 \mathrm{H}, \mathrm{m}), 1.64(1 \mathrm{H}, \mathrm{d}, J=6.4 \mathrm{~Hz}, \mathrm{OH}), 1.60(1 \mathrm{H}, \mathrm{dddd}, J=14.0,9.5, .6 .6$, $5.3 \mathrm{~Hz}), 1.05(9 \mathrm{H}, \mathrm{s}), 1.00(9 \mathrm{H}, \mathrm{s}), 0.97-0.85(13 \mathrm{H}, \mathrm{m}), 0.65-0.58(4 \mathrm{H}, \mathrm{m}) ;{ }^{13} \mathrm{C}$ NMR for hydroxy ketone form $\mathbf{S 3}\left(150 \mathrm{MHz}, \mathrm{CDCl}_{3}\right) \delta 212.0,138.6,138.4,128.4,128.3,127.8,127.64,127.58,127.4,82.3,81.9$, $81.8,75.8,74.9,74.8,72.9,71.5,70.9,69.2,67.1,43.0,38.1,35.1,29.6,27.5,27.1,23.9,22.6,19.9$, 
17.21, 17.18, 12.9, 7.0, 6.9, 4.0, 3.8; HRDARTMS $m / z$ calcd for $\mathrm{C}_{44} \mathrm{H}_{76} \mathrm{O}_{9} \mathrm{NSi}_{2}\left(\mathrm{M}^{+} \mathrm{NH}_{4}^{+}\right)$818.5053, found 818.5058 .

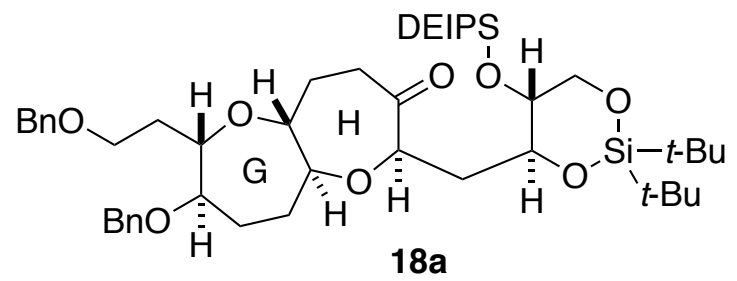

Seven-membered ketone 18a. (i) Ring expansion reaction. To a solution of the six-membered ketone 5 (1.72 g, $2.19 \mathrm{mmol})$ and MS4 $\AA$ (11.0 g, $50 \mathrm{mg}$ per $1 \mathrm{~mL}$ solvent) in $\mathrm{CH}_{2} \mathrm{Cl}_{2}(220 \mathrm{~mL})$ at $-80{ }^{\circ} \mathrm{C}$ was added $\mathrm{BF}_{3} \cdot \mathrm{OEt}_{2}\left(1.40 \mathrm{~mL}, 11.0 \mathrm{mmol}, 5.0\right.$ equiv) and $\mathrm{TMSCHN}_{2}(2.0 \mathrm{M}$ solution in $n$-hexane, $5.5 \mathrm{~mL}$, $11.0 \mathrm{mmol}, 5.0$ equiv). The reaction mixture was stirred at $-80^{\circ} \mathrm{C}$ for $1 \mathrm{~h}$, and the reaction was quenched with saturated aqueous $\mathrm{NaHCO}_{3}$ solution. MS4 $\AA$ was removed by filtration through a Celite pad, and the filtrate was extracted with $\mathrm{CH}_{2} \mathrm{Cl}_{2}$. The extract was washed with brine, dried, and concentrated under reduced pressure. The resulting pale yellow oil of $\alpha$-trimethylsilyl ketone $(1.87 \mathrm{~g})$ was subjected to the next methanolysis reaction without further purification.

(ii) Methanolysis. To a solution of the above $\alpha$-trimethylsilyl ketone $(1.87 \mathrm{~g})$ in $\mathrm{MeOH}(128 \mathrm{~mL})$ and $\mathrm{CH}_{2} \mathrm{Cl}_{2}(128 \mathrm{~mL})$ was added PPTS (550 mg, $2.19 \mathrm{mmol}, 1.0$ equiv). The reaction mixture was stirred at room temperature for $24 \mathrm{~h}$, and the reaction was quenched with $\mathrm{Et}_{3} \mathrm{~N}$. The resulting mixture was concentrated under reduced pressure. Flash chromatography on silica gel $(10 \rightarrow 30 \%$ EtOAc in $n$-hexane $)$ afforded seven-membered ketone 18a (829 mg, 47\%) and hydroxy ketone 18b (600 mg, 41\%).

Seven-membered ketone 18a. Colorless oil; $[\alpha]^{22} \mathrm{D}-14.4$ (c 1.74, $\left.\mathrm{CHCl}_{3}\right)$; IR (film) 2933, 2861, 1716, $1091 \mathrm{~cm}^{-1} ;{ }^{1} \mathrm{H}$ NMR $\left(600 \mathrm{MHz}, \mathrm{CDCl}_{3}\right) \delta$ 7.36-7.31 (8H, m), 7.30-7.26 (2H, m), 4.59 and $4.38($ each $1 \mathrm{H}$, d, $J=11.7 \mathrm{~Hz}), 4.56$ and $4.42($ each $1 \mathrm{H}, \mathrm{d}, J=11.9 \mathrm{~Hz}), 4.02(1 \mathrm{H}, \mathrm{ddd}, J=10.3,9.0,2.0 \mathrm{~Hz}), 3.97(1 \mathrm{H}$, $\mathrm{dd}, J=7.5,2.8 \mathrm{~Hz}), 3.96(1 \mathrm{H}, \mathrm{dd}, J=10.4,4.4 \mathrm{~Hz}), 3.74(1 \mathrm{H}, \mathrm{t}, J=10.4 \mathrm{~Hz}), 3.63(1 \mathrm{H}, \mathrm{ddd}, J=9.9,6.6$, $2.9 \mathrm{~Hz}), 3.58(1 \mathrm{H}, \mathrm{td}, J=8.9,5.6 \mathrm{~Hz}), 3.51(1 \mathrm{H}, \mathrm{ddd}, J=9.5,6.4,4.4 \mathrm{~Hz}), 3.49(1 \mathrm{H}, \mathrm{ddd}, J=9.9,9.0$, $4.6 \mathrm{~Hz}), 3.43(1 \mathrm{H}, \mathrm{dt}, J=6.4,3.5 \mathrm{~Hz}), 3.39(1 \mathrm{H}, \mathrm{ddd}, J=11.0,9.5,4.3 \mathrm{~Hz}), 3.04(1 \mathrm{H}, \mathrm{td}, J=9.6,4.5 \mathrm{~Hz})$, 
$2.97(1 \mathrm{H}, \mathrm{ddd}, J=13.8,12.2,2.0 \mathrm{~Hz}), 2.27(1 \mathrm{H}, \mathrm{ddd}, J=13.8,7.4,2.2 \mathrm{~Hz}), 2.22(1 \mathrm{H}, \mathrm{ddd}, J=12.1,7.2$, $0.8 \mathrm{~Hz}), 2.07-1.95(3 \mathrm{H}, \mathrm{m}), 1.92-1.83(3 \mathrm{H}, \mathrm{m}), 1.67(1 \mathrm{H}, \mathrm{ddd}, J=14.9,12.2,3.3 \mathrm{~Hz}), 1.59(1 \mathrm{H}, \mathrm{dddd}, J$ $=14.5,10.1,5.5,4.6 \mathrm{~Hz}), 1.45(1 \mathrm{H}, \mathrm{dddd}, J=14.2,13.6,11.4,0.7 \mathrm{~Hz}), 1.04(9 \mathrm{H}, \mathrm{s}), 0.979(3 \mathrm{H}, \mathrm{d}, J=$ $7.0 \mathrm{~Hz}), 0.976(3 \mathrm{H}, \mathrm{d}, J=7.0 \mathrm{~Hz}), 0.973(3 \mathrm{H}, \mathrm{t}, J=7.9 \mathrm{~Hz}), 0.966(3 \mathrm{H}, \mathrm{t}, J=8.1 \mathrm{~Hz}), 0.93(9 \mathrm{H}, \mathrm{s}), 0.91$ $(1 \mathrm{H}, \mathrm{m}), 0.66-0.57(4 \mathrm{H}, \mathrm{m}) ;{ }^{13} \mathrm{C} \mathrm{NMR}\left(150 \mathrm{MHz}, \mathrm{CDCl}_{3}\right) \delta 216.7,138.5,138.4,128.34,128.30,127.8$, $127.65,127.56,127.5,87.1,85.4,83.6,82.3,81.2,74.7,72.9,71.6,70.9,68.9,66.8,38.9,37.3,35.0,30.8$, 28.5, 27.6, 26.9, 24.3, 22.6, 19.8, 17.21, 17.19, 12.9, 7.0, 6.9, 4.0, 3.8; HRDARTMS m/z calcd for $\mathrm{C}_{45} \mathrm{H}_{73} \mathrm{O}_{8} \mathrm{Si}_{2}\left(\mathrm{MH}^{+}\right)$797.4838, found 797.4844.

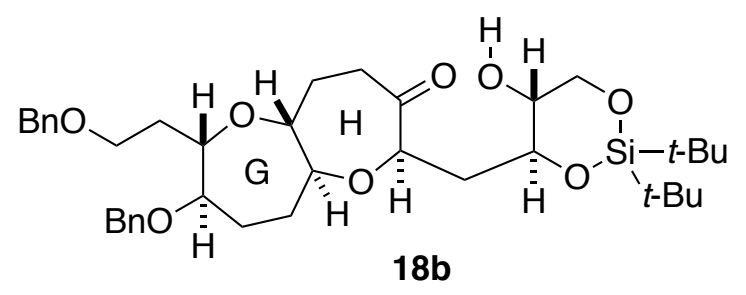

Hydroxy ketone $\mathbf{1 8 b}$ (84:16 mixture of tautomers of the hydroxy ketone form $\mathbf{1 8 b}$ and the hemiacetal form 18b'). Colorless oil; $[\alpha]^{28}{ }_{\mathrm{D}}+1.1\left(c 0.61, \mathrm{CHCl}_{3}\right)$; IR (film) 3437, 2933, 2861, 1716, $1105 \mathrm{~cm}^{-1} ;{ }^{1} \mathrm{H}$

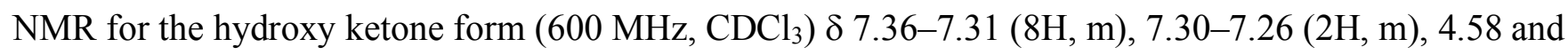
$4.37($ each $1 \mathrm{H}, \mathrm{d}, J=11.6 \mathrm{~Hz}), 4.56$ and $4.42($ each $1 \mathrm{H}, \mathrm{d}, J=12.1 \mathrm{~Hz}), 4.13(1 \mathrm{H}, \mathrm{dd}, J=7.1,3.9 \mathrm{~Hz})$, $4.12(1 \mathrm{H}, \mathrm{dd}, J=10.3,4.8 \mathrm{~Hz}), 4.04(1 \mathrm{H}, \mathrm{ddd}, J=9.3,5.5,3.8 \mathrm{~Hz}), 3.78(1 \mathrm{H}, \mathrm{t}, J=10.3 \mathrm{~Hz}), 3.67(1 \mathrm{H}$, $\mathrm{dt}, J=9.5,4.6 \mathrm{~Hz}), 3.63(1 \mathrm{H}, \mathrm{ddd}, J=9.8,6.3,3.1 \mathrm{~Hz}), 3.57(1 \mathrm{H}, \mathrm{td}, J=9.0,5.5 \mathrm{~Hz}), 3.50(1 \mathrm{H}, \mathrm{ddd}, J=$ 9.2, 6.2, $4.4 \mathrm{~Hz}), 3.44(1 \mathrm{H}, \mathrm{dt}, J=6.2,3.5 \mathrm{~Hz}), 3.39(1 \mathrm{H}, \mathrm{ddd}, J=11.0,9.5,4.4 \mathrm{~Hz}), 3.10(1 \mathrm{H}, \mathrm{td}, J=9.7$, $4.5 \mathrm{~Hz}), 2.85(1 \mathrm{H}, \mathrm{ddd}, J=14.1,12.2,2.2 \mathrm{~Hz}), 2.45(1 \mathrm{H}, \mathrm{d}, J=4.8 \mathrm{~Hz}), 2.27(1 \mathrm{H}, \mathrm{ddd}, J=12.2,7.0,1.1$ Hz), $2.10(1 \mathrm{H}, \mathrm{dt}, J=14.7,3.7 \mathrm{~Hz}), 2.07-1.98(3 \mathrm{H}, \mathrm{m}), 1.97-1.85(3 \mathrm{H}, \mathrm{m}), 1.68(1 \mathrm{H}, \mathrm{ddd}, J=14.7,11.9$, $2.8 \mathrm{~Hz}), 1.58(1 \mathrm{H}$, dddd, $J=14.3,9.7,5.5,4.7 \mathrm{~Hz}), 1.48(1 \mathrm{H}$, dddd, $J=14.7,13.6,11.4,1.1 \mathrm{~Hz}), 1.03$ $(9 \mathrm{H}, \mathrm{s}), 0.98(9 \mathrm{H}, \mathrm{s}) ;{ }^{13} \mathrm{C} \mathrm{NMR}$ for the hydroxy ketone form $\left(150 \mathrm{MHz}, \mathrm{CDCl}_{3}\right) \delta 216.0,138.4,138.3$, $128.4,128.3,127.9,127.7,127.64,127.58,87.0,84.8,83.1,81.9,81.2,75.0,72.9,70.9,69.7,68.6,66.7$, 37.6, 37.1, 34.9, 30.7, 28.3, 27.5, 27.1, 24.0, 22.6, 20.1; ${ }^{1} \mathrm{H}$ NMR for the hemiacetal form $(600 \mathrm{MHz}$, $\left.\mathrm{CDCl}_{3}\right) \delta 7.36-7.31(8 \mathrm{H}, \mathrm{m}), 7.30-7.26(2 \mathrm{H}, \mathrm{m}), 4.56$ and 4.42 (each $\left.1 \mathrm{H}, \mathrm{d}, J=11.7 \mathrm{~Hz}\right), 4.52$ and 4.44 
(each 1H, d, $J=11.7 \mathrm{~Hz}), 4.07(1 \mathrm{H}, \mathrm{dd}, J=9.0,3.9 \mathrm{~Hz}), 3.81-3.72(3 \mathrm{H}, \mathrm{m}), 3.69-3.61(1 \mathrm{H}, \mathrm{m}), 3.59-$ $3.53(2 \mathrm{H}, \mathrm{m}), 3.52-3.47(1 \mathrm{H}, \mathrm{m}), 3.39-3.31(3 \mathrm{H}, \mathrm{m}), 2.62(1 \mathrm{H}, \mathrm{br} \mathrm{s}), 2.19(1 \mathrm{H}, \mathrm{dt}, J=12.0,4.4 \mathrm{~Hz}), 1.99-$ $1.79(7 \mathrm{H}, \mathrm{m}), 1.76-1.69(2 \mathrm{H}, \mathrm{m}), 1.65-1.57(2 \mathrm{H}, \mathrm{m}), 1.03(9 \mathrm{H}, \mathrm{s}), 0.98(9 \mathrm{H}, \mathrm{s}) ;{ }^{13} \mathrm{C}$ NMR peaks for the hemiacetal form $\mathbf{1 8 b ^ { \prime }}$ could not be fully assigned because of too weak signals; HRDARTMS $\mathrm{m} / z$ calcd for $\mathrm{C}_{38} \mathrm{H}_{57} \mathrm{O}_{8} \mathrm{Si}\left(\mathrm{MH}^{+}\right)$669.3817, found 669.3813.

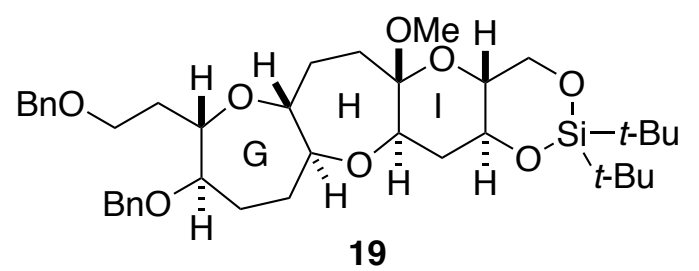

Methyl acetal 19. To a solution of the seven-membered ketone 18a (3.76 g, $4.72 \mathrm{mmol})$ in a mixed solvent of $\mathrm{CH}_{2} \mathrm{Cl}_{2}-\mathrm{MeOH}-\mathrm{CH}(\mathrm{OMe})_{3}(10: 1: 10)(190 \mathrm{~mL})$ was added $\mathrm{TsOH} \cdot \mathrm{H}_{2} \mathrm{O}(2.69 \mathrm{~g}, 14.2 \mathrm{mmol}, 3.2$ equiv), and the reaction mixture was stirred at room temperature for $6 \mathrm{~h}$. The reaction was quenched with $\mathrm{Et}_{3} \mathrm{~N}$, and the resulting mixture was concentrated under reduced pressure. Flash chromatography on silica gel $\left(20 \rightarrow 30 \% \mathrm{Et}_{2} \mathrm{O}\right.$ in $n$-hexane $)$ afforded methyl acetal $19(3.07 \mathrm{~g}, 95 \%)$ as a colorless oil. $[\alpha]^{22} \mathrm{D}-10.7$ (c 1.31, $\left.\mathrm{CHCl}_{3}\right)$; IR (film) 2933, 2858, 1088, $1057 \mathrm{~cm}^{-1} ;{ }^{1} \mathrm{H} \mathrm{NMR}\left(600 \mathrm{MHz}, \mathrm{CDCl}_{3}\right) \delta 7.34-7.25(10 \mathrm{H}$, $\mathrm{m}), 4.56$ and $4.36($ each $1 \mathrm{H}, \mathrm{d}, J=11.7 \mathrm{~Hz}), 4.51$ and $4.45($ each $1 \mathrm{H}, \mathrm{d}, J=11.9 \mathrm{~Hz}), 4.05(1 \mathrm{H}, \mathrm{dd}, J=$ 10.0, 4.9 Hz), $3.79(1 \mathrm{H}, \mathrm{t}, J=10.3 \mathrm{~Hz}), 3.76(1 \mathrm{H}, \mathrm{ddd}, J=11.0,9.4,4.6 \mathrm{~Hz}), 3.65-3.61(2 \mathrm{H}, \mathrm{m}), 3.57$ $(1 \mathrm{H}, \mathrm{ddd}, J=9.3,8.1,6.2 \mathrm{~Hz}), 3.54(1 \mathrm{H}, \mathrm{ddd}, J=9.3,7.2,4.8 \mathrm{~Hz}), 3.46(1 \mathrm{H}, \mathrm{ddd}, J=10.5,9.6,4.9 \mathrm{~Hz})$ 3.36-3.33 (2H, m), $3.32(1 \mathrm{H}, \mathrm{dd}, J=12.1,4.2 \mathrm{~Hz}), 3.22(3 \mathrm{H}, \mathrm{s}), 2.12(1 \mathrm{H}, \mathrm{dt}, J=11.6,4.4 \mathrm{~Hz}), 1.98-$ $1.92(5 \mathrm{H}, \mathrm{m}), 1.92(1 \mathrm{H}, \mathrm{q}, J=11.6 \mathrm{~Hz}), 1.81(1 \mathrm{H}, \mathrm{m}), 1.75-1.69(2 \mathrm{H}, \mathrm{m}), 1.64-1.57(2 \mathrm{H}, \mathrm{m}), 1.03(9 \mathrm{H}$ s), $0.98(9 \mathrm{H}, \mathrm{s}) ;{ }^{13} \mathrm{C} \mathrm{NMR}\left(150 \mathrm{MHz}, \mathrm{CDCl}_{3}\right) \delta 138.6,138.5,128.31,128.29,127.62,127.59,127.5(\mathrm{x} 2)$ $99.8,84.7,83.1,82.3,81.7,81.4,73.1,72.9,70.7,69.5,67.2,67.1,47.4,35.0,34.7,29.5,28.5,28.2,27.4$ 27.0, 24.0, 22.6, 19.9; HRDARTMS m/z calcd for $\mathrm{C}_{39} \mathrm{H}_{62} \mathrm{O}_{8} \mathrm{NSi}\left(\mathrm{M}+\mathrm{NH}_{4}{ }^{+}\right)$700.4239, found 700.4239. 
Methyl acetal from hydroxy ketone 18b. To a solution of hydroxy ketone $\mathbf{1 8 b}(2.60 \mathrm{~g}, 3.89 \mathrm{mmol})$ in a mixed solvent of $\mathrm{CH}_{2} \mathrm{Cl}_{2}-\mathrm{CH}(\mathrm{OMe})_{3}(1: 1)(160 \mathrm{~mL})$ was added $\mathrm{TsOH} \cdot \mathrm{H}_{2} \mathrm{O}(2.37 \mathrm{~g}, 12.4 \mathrm{mmol}, 3.2$ equiv), and the reaction mixture was stirred at room temperature for $1 \mathrm{~h}$. The reaction was quenched with $\mathrm{Et}_{3} \mathrm{~N}$, and the resulting mixture was concentrated under reduced pressure. Flash chromatography on silica gel $(10 \rightarrow 20 \%$ EtOAc in $n$-hexane) afforded methyl acetal $19(1.98 \mathrm{~g}, 75 \%)$ as a colorless oil.

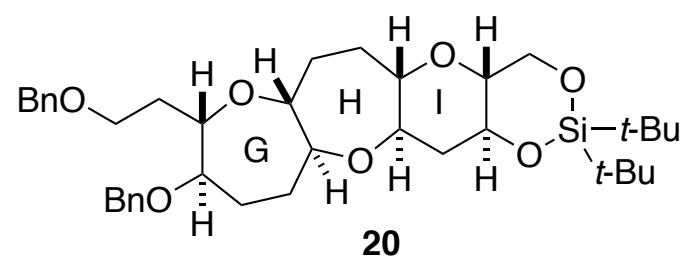

Tricyclic ether 20. To a solution of methyl acetal $19(1.98 \mathrm{~g}, 2.91 \mathrm{mmol})$ in $\mathrm{CH}_{2} \mathrm{Cl}_{2}(145 \mathrm{~mL})$ at $0{ }^{\circ} \mathrm{C}$ was added $\mathrm{Et}_{3} \mathrm{SiH}$ (4.60 mL, $29.1 \mathrm{mmol}, 10$ equiv) and TMSOTf (2.60 mL, $14.5 \mathrm{mmol}, 5.0$ equiv), and the reaction mixture was stirred at $0{ }^{\circ} \mathrm{C}$ for $40 \mathrm{~min}$. The reaction was quenched with saturated aqueous $\mathrm{NaHCO}_{3}$ solution and the resulting mixture was extracted with $\mathrm{CH}_{2} \mathrm{Cl}_{2}$. The extract was washed with brine, dried, and concentrated under reduced pressure. Flash chromatography on silica gel $(5 \rightarrow 10 \%$ EtOAc in $n$-hexane) afforded tricyclic ether $\mathbf{2 0}(1.77 \mathrm{~g}, 93 \%)$ as a colorless solid. $\mathrm{Mp} 79-81{ }^{\circ} \mathrm{C} ;[\alpha]^{22} \mathrm{D}-$ 36.5 ( c 0.69, $\left.\mathrm{CHCl}_{3}\right)$; IR (film) 2932, 2858, $1088 \mathrm{~cm}^{-1} ;{ }^{1} \mathrm{H}$ NMR $\left(600 \mathrm{MHz}, \mathrm{CDCl}_{3}\right) \delta 7.35-7.25(10 \mathrm{H}$, m), 4.55 and $4.36($ each $1 \mathrm{H}, \mathrm{d}, J=11.7 \mathrm{~Hz}), 4.52$ and $4.44($ each $1 \mathrm{H}, \mathrm{d}, J=11.9 \mathrm{~Hz}), 4.12(1 \mathrm{H}, \mathrm{dd}, J=$ 10.1, $5.0 \mathrm{~Hz}), 3.78(1 \mathrm{H}, \mathrm{t}, J=10.3 \mathrm{~Hz}), 3.75(1 \mathrm{H}, \mathrm{ddd}, J=11.0,9.2,4.5 \mathrm{~Hz}), 3.64(1 \mathrm{H}, \mathrm{ddd}, J=9.6,4.8$, $3.8 \mathrm{~Hz}), 3.56(1 \mathrm{H}, \mathrm{ddd}, J=9.4,8.2,6.1 \mathrm{~Hz}), 3.52(1 \mathrm{H}, \mathrm{ddd}, J=9.4,6.8,4.8 \mathrm{~Hz}), 3.45(1 \mathrm{H}, \mathrm{dt}, J=9.0$, $6.0 \mathrm{~Hz}), 3.41(1 \mathrm{H}, \mathrm{td}, J=5.3,2.6 \mathrm{~Hz}), 3.32(1 \mathrm{H}, \mathrm{td}, J=9.1,4.5 \mathrm{~Hz}), 3.24(1 \mathrm{H}, \mathrm{ddd}, J=10.3,9.4,5.0 \mathrm{~Hz})$, $3.22(1 \mathrm{H}, \mathrm{ddd}, J=11.6,9.2,4.0 \mathrm{~Hz}), 3.12(1 \mathrm{H}, \mathrm{td}, J=8.5,4.2 \mathrm{~Hz}), 2.41(1 \mathrm{H}, \mathrm{dt}, J=11.9,4.3 \mathrm{~Hz}), 1.95$ (1H, dddd, $J=14.7,7.7,6.0,1.3 \mathrm{~Hz}), 1.91-1.72(7 \mathrm{H}, \mathrm{m}), 1.67$ (1H, dddd, $J=14.3,11.7,2.2,1.6 \mathrm{~Hz})$, $1.60(1 \mathrm{H}, \mathrm{dddd}, J=14.3,9.5,6.0,5.0 \mathrm{~Hz}), 1.52(1 \mathrm{H}, \mathrm{q}, J=11.4 \mathrm{~Hz}), 1.03(9 \mathrm{H}, \mathrm{s}), 0.97(9 \mathrm{H}, \mathrm{s}) ;{ }^{13} \mathrm{C} \mathrm{NMR}$ $\left(150 \mathrm{MHz}, \mathrm{CDCl}_{3}\right) \delta 138.52,138.45,128.3(\mathrm{x} 2), 127.7,127.6,127.5$ (x2), 84.3, 83.5, 82.2, 82.1, 81.1, 
$79.8,77.3,72.9,72.8,70.7,67.1,66.9,40.4,34.9,30.3,28.4,28.1,27.4,27.0,23.8,22.6,19.9$;

HRDARTMS $m / z$ calcd for $\mathrm{C}_{38} \mathrm{H}_{57} \mathrm{O}_{7} \mathrm{Si}\left(\mathrm{MH}^{+}\right)$653.3868, found 653.3873 .

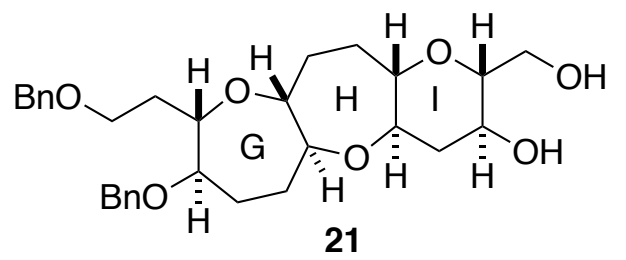

Diol 21. To a solution of silylene 20 (318 $\mathrm{mg}, 0.487 \mathrm{mmol})$ in THF $(5 \mathrm{~mL})$ was added TBAF $(1.0 \mathrm{M}$ solution in THF, $1.0 \mathrm{~mL}, 1.0 \mathrm{mmol}, 2.05$ equiv). The reaction mixture was stirred at room temperature for $2 \mathrm{~h}$, and the reaction was quenched with saturated aqueous $\mathrm{NH}_{4} \mathrm{Cl}$ solution. The resulting mixture was extracted with EtOAc, and the extract was washed with water and brine, dried, and concentrated under reduced pressure. Flash chromatography on silica gel (EtOAc) afforded diol 21 (229 mg, 91\%) as a colorless solid. Mp $92-94{ }^{\circ} \mathrm{C} ;[\alpha]^{22} \mathrm{D}-38.5\left(\mathrm{c} 1.42, \mathrm{CHCl}_{3}\right)$; IR (film) 3418, 2934, 2866, $1061 \mathrm{~cm}^{-1} ;{ }^{1} \mathrm{H}$ $\operatorname{NMR}\left(600 \mathrm{MHz}, \mathrm{CDCl}_{3}\right) \delta 7.35-7.25(10 \mathrm{H}, \mathrm{m}), 4.54$ and $4.36($ each 1H, d, $J=11.7 \mathrm{~Hz}), 4.52$ and 4.45 (each $1 \mathrm{H}, \mathrm{d}, J=11.9 \mathrm{~Hz}), 3.82(1 \mathrm{H}, \mathrm{dd}, J=11.5,3.9 \mathrm{~Hz}), 3.73(1 \mathrm{H}, \mathrm{dd}, J=11.5,5.1 \mathrm{~Hz}), 3.65(1 \mathrm{H}, \mathrm{dt}, J$ $=9.6,4.2 \mathrm{~Hz}), 3.61(1 \mathrm{H}, \mathrm{ddd}, J=11.1,9.4,5.0 \mathrm{~Hz}), 3.56(1 \mathrm{H}, \mathrm{ddd}, J=9.2,8.3,6.1 \mathrm{~Hz}), 3.52(1 \mathrm{H}, \mathrm{ddd}$, $J=9.2,6.8,4.8 \mathrm{~Hz}), 3.47(1 \mathrm{H}, \mathrm{dt}, J=9.0,5.8 \mathrm{~Hz}), 3.41(1 \mathrm{H}, \mathrm{ddd}, J=5.6,4.8,2.5 \mathrm{~Hz}), 3.32(1 \mathrm{H}, \mathrm{td}, J=$ 9.3, 4.6 Hz), 3.17 (1H, ddd, $J=11.4,9.1,4.0 \mathrm{~Hz}), 3.13(1 \mathrm{H}, \mathrm{ddd}, J=9.2,5.0,4.4 \mathrm{~Hz}), 3.08(1 \mathrm{H}, \mathrm{ddd}, J$ $=9.0,7.9,4.1 \mathrm{~Hz}), 2.41(1 \mathrm{H}, \mathrm{br} \mathrm{s}), 2.36(1 \mathrm{H}, \mathrm{dt}, J=11.6,4.4 \mathrm{~Hz}), 2.29(1 \mathrm{H}, \mathrm{br} \mathrm{s}), 1.95(1 \mathrm{H}, \mathrm{dddd}, J=$ 14.7, 7.2, 6.1, 1.1 Hz), 1.92-1.84 (4H, m), 1.83-1.74 (3H, m), 1.66 (1H, ddt, $J=14.1,12.2,2.0 \mathrm{~Hz}), 1.61$ $(1 \mathrm{H}, \mathrm{dddd}, J=14.1,9.9,5.7,5.0 \mathrm{~Hz}), 1.50(1 \mathrm{H}, \mathrm{q}, J=11.6 \mathrm{~Hz}) ;{ }^{13} \mathrm{C} \mathrm{NMR}\left(150 \mathrm{MHz}, \mathrm{CDCl}_{3}\right) \delta 138.5$, $138.4,128.33,128.32,127.7,127.6,127.52,127.51,84.2,83.3,82.0,81.9,81.1,81.0,79.7,72.9,70.7$, 67.2, 67.1, 63.2, 40.4, 34.9, 30.2, 28.3, 28.0, 23.8; HRDARTMS $m / z$ calcd for $\mathrm{C}_{30} \mathrm{H}_{41} \mathrm{O}_{7}\left(\mathrm{MH}^{+}\right)$513.2847, found 513.2848 . 


\section{Synthesis of the seven-membered model ketone 22}

Scheme S1. Synthesis of seven-membered ketone 33.
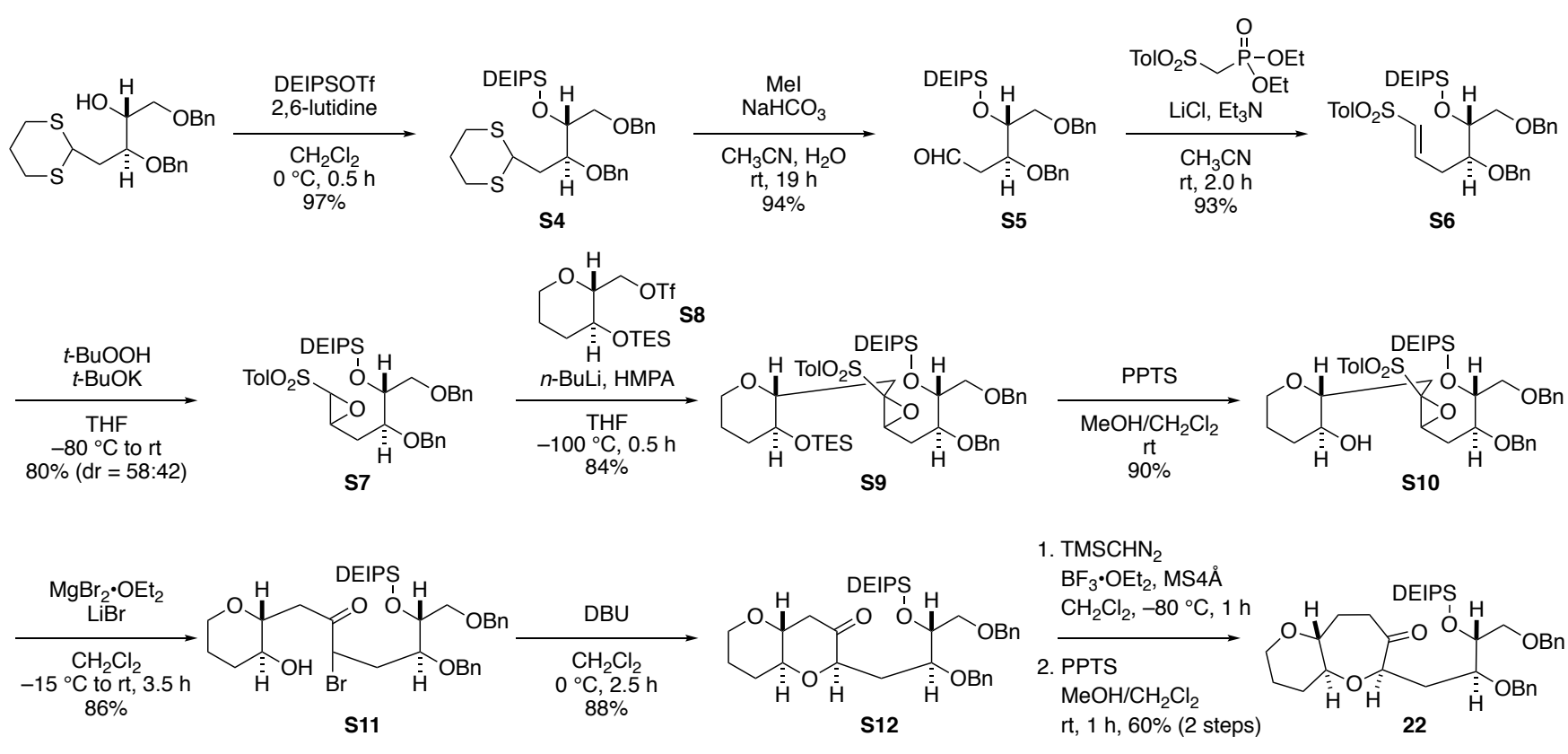<smiles>Brc1ccccc1</smiles>

S4

DEIPS ether S4. To a solution of alcohol (2R,3S)-1,3-bis(benzyloxy)-4-(1,3-dithian-2-yl)butan-2-ol ${ }^{3}$ (8.78 g, $21.7 \mathrm{mmol})$ in $\mathrm{CH}_{2} \mathrm{Cl}_{2}(22 \mathrm{~mL})$ and 2,6-lutidine $(3.77 \mathrm{~mL}, 32.6 \mathrm{mmol})$ at $0{ }^{\circ} \mathrm{C}$ was added DEIPSOTf $(5.74 \mathrm{~mL}, 23.9 \mathrm{mmol})$, and the reaction mixture was stirred at $0{ }^{\circ} \mathrm{C}$ for $0.5 \mathrm{~h}$. The reaction was quenched with saturated aqueous $\mathrm{NaHCO}_{3}$ solution and the reaction mixture was extracted with EtOAchexane (1:1). The extract was washed with water and brine, dried, and concentrated under reduced pressure. Purification by flash chromatography (5\% EtOAc in hexane) afforded DEIPS ether S4 (11.21 g, 97\%) as a colorless oil. [ $\alpha]^{25} \mathrm{D}-21.9\left(c\right.$ 0.42, $\left.\mathrm{CHCl}_{3}\right)$; IR (film) 2950, 2895, 2873, $1098 \mathrm{~cm}^{-1}$; ${ }^{1} \mathrm{H}$ NMR $\left(600 \mathrm{MHz} \mathrm{CDCl}_{3}\right) \delta 7.34-7.32(8 \mathrm{H}, \mathrm{m}), 7.30-7.26(2 \mathrm{H}, \mathrm{m}), 4.72$ and $4.54($ each $1 \mathrm{H}, \mathrm{d}, J=11.4 \mathrm{~Hz})$, 4.52 and 4.51 (each $1 \mathrm{H}, \mathrm{d}, J=11.9 \mathrm{~Hz}), 4.10(1 \mathrm{H}, \mathrm{d}, J=10.5,4.2 \mathrm{~Hz}), 4.00(1 \mathrm{H}, \mathrm{td}, J=5.5,2.9 \mathrm{~Hz}), 3.90$ $(1 \mathrm{H}, \mathrm{dt}, J=9.9,2.8 \mathrm{~Hz}), 3.51(1 \mathrm{H}, \mathrm{dd}, J=9.9,5.5 \mathrm{~Hz}), 3.50(1 \mathrm{H}, \mathrm{dd}, J=9.9,5.5 \mathrm{~Hz}), 2.83(1 \mathrm{H}, \mathrm{ddd}, J=$ 14.1, 10.4, 2.9 Hz), $2.81(1 \mathrm{H}, \mathrm{ddd}, J=14.1,5.0,4.2 \mathrm{~Hz}), 2.78(1 \mathrm{H}, \mathrm{ddd}, J=14.1,5.0,3.1 \mathrm{~Hz}), 2.69(1 \mathrm{H}$, 
ddd, $J=14.1,11.4,2.6 \mathrm{~Hz}), 2.08(1 \mathrm{H}, \mathrm{dtdd}, J=14.1,5.0,2.9,2.6 \mathrm{~Hz}), 2.04(1 \mathrm{H}, \mathrm{ddd}, J=14.5,9.9,4.2$ Hz), 1.91 (1H, ddd, $J=14.7,10.6,2.9 \mathrm{~Hz}), 1.86(1 \mathrm{H}$, ddddd, $J=14.1,11.2,10.4,4.2,3.1 \mathrm{~Hz}), 0.99-0.93$ $(7 \mathrm{H}, \mathrm{m}), 0.96(3 \mathrm{H}, \mathrm{t}, J=7.9 \mathrm{~Hz}), 0.95(3 \mathrm{H}, \mathrm{t}, J=7.9 \mathrm{~Hz}), 0.67-0.59(4 \mathrm{H}, \mathrm{m}) ;{ }^{13} \mathrm{C}$ NMR $(150 \mathrm{MHz}$, $\left.\mathrm{CDCl}_{3}\right) \delta 138.8,138.2,128.3,128.2,128.0,127.7,127.5,127.4,77.8,73.5,73.4,73.0,71.9,44.3,36.7$, 30.3, 29.8, 26.0, $17.3(\times 2), 13.0,7.10,7.08,3.80,3.77$; HRFABMS $m / z$ calcd for $\mathrm{C}_{29} \mathrm{H}_{45} \mathrm{O}_{3} \mathrm{~S} \mathrm{~S}_{2} \mathrm{Si}\left(\mathrm{MH}^{+}\right)$ 533.2579, found 533.2576.

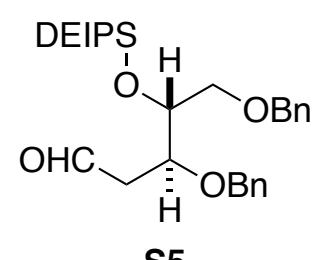

S5

Aldehyde S5. To a solution of dithioacetal S4 (7.95 g, $14.9 \mathrm{mmol})$ in $\mathrm{MeCN}(187 \mathrm{~mL})$ and water $(27$ $\mathrm{mL})$ were added $\mathrm{NaHCO}_{3}(25.1 \mathrm{~g}, 298 \mathrm{mmol})$ and $\mathrm{MeI}(27.9 \mathrm{~mL}, 448 \mathrm{mmol})$, and the suspension was stirred vigorously at room temperature for $19 \mathrm{~h}$. The reaction mixture was concentrated to half of the volume under reduced pressure, and extracted with EtOAc. The extract was washed with saturated aqueous $\mathrm{Na}_{2} \mathrm{~S}_{2} \mathrm{O}_{3}$ solution, water, and brine, dried, and concentrated under reduced pressure. Purification by flash chromatography (8\% EtOAc in hexane) afforded aldehyde $\mathbf{S 5}(6.21 \mathrm{~g}, 94 \%)$ as a colorless oil. $[\alpha]^{25} \mathrm{D}-19.5$ (c 1.11, $\left.\mathrm{CHCl}_{3}\right)$; IR (film) 2952, 2911, 2874, 1725, $1099 \mathrm{~cm}^{-1} ;{ }^{1} \mathrm{H}$ NMR (600 MHz, $\left.\mathrm{CDCl}_{3}\right)$ $\delta 9.76(1 \mathrm{H}, \mathrm{dd}, J=2.6,1.7 \mathrm{~Hz}), 7.37-7.24(10 \mathrm{H}, \mathrm{m}), 4.62$ and $4.54($ each $1 \mathrm{H}, \mathrm{d}, J=11.4 \mathrm{~Hz}), 4.49(2 \mathrm{H}$, s), $4.10(1 \mathrm{H}, \mathrm{dt}, J=7.4,3.9 \mathrm{~Hz}), 4.05(1 \mathrm{H}, \mathrm{ddd}, J=6.0,4.9,3.7 \mathrm{~Hz}), 3.50(1 \mathrm{H}, \mathrm{dd}, J=9.7,4.8 \mathrm{~Hz}), 3.45$ (1H, dd, $J=9.7,5.9 \mathrm{~Hz}), 2.69(1 \mathrm{H}, \mathrm{ddd}, J=16.7,7.3,2.6 \mathrm{~Hz}), 2.60(1 \mathrm{H}, \mathrm{ddd}, J=16.7,4.2,1.7 \mathrm{~Hz})$, 0.99-0.89 (13H, m), 0.67-0.59 (4H, m); ${ }^{13} \mathrm{C}$ NMR $\left(150 \mathrm{MHz}, \mathrm{CDCl}_{3}\right) \delta 201.7,138.1,137.9,128.4,128.3$, $127.9,127.75,127.69,127.65,75.9,73.4,72.7,72.3,71.5,44.5,17.3(\times 2), 12.9,7.00,6.98,3.78,3.75$; HRFABMS $m / z$ calcd for $\mathrm{C}_{26} \mathrm{H}_{38} \mathrm{O}_{4} \mathrm{SiNa}\left(\mathrm{MNa}^{+}\right)$465.2437, found 465.2439 . 


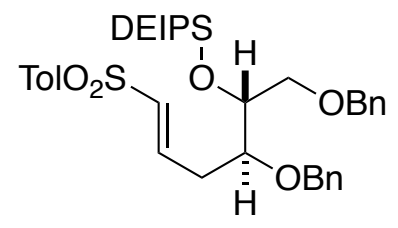

S6

Vinyl sulfone S6. To a solution of aldehyde $\mathbf{S 5}(6.21 \mathrm{~g}, 14.0 \mathrm{mmol})$, diethyl $p$ tolylsulfonylmethylphosphonate (5.16 g, $16.8 \mathrm{mmol})$, and $\mathrm{LiCl}(713 \mathrm{mg}, 16.8 \mathrm{mmol})$ in $\mathrm{MeCN}(83 \mathrm{~mL})$ was added $\mathrm{Et}_{3} \mathrm{~N}(2.35 \mathrm{~mL}, 16.8 \mathrm{mmol})$, and the reaction mixture was stirred at room temperature for 2.0 h. The reaction was quenched with saturated aqueous $\mathrm{NH}_{4} \mathrm{Cl}$ solution. The reaction mixture was extracted with EtOAc, and the extract was washed with water and brine, dried, and concentrated under reduced pressure. Purification by flash chromatography (15\% EtOAc in hexane) afforded vinyl sulfone S6 (7.81 g, 93\%) as a colorless oil. $[\alpha]^{26} \mathrm{D}-10.2\left(c 1.14, \mathrm{CHCl}_{3}\right) ; \mathrm{IR}$ (film) 2952, 2911, 2873, 1145, $1088 \mathrm{~cm}^{-1} ;{ }^{1} \mathrm{H}$ NMR (600 MHz, $\left.\mathrm{CDCl}_{3}\right) \delta 7.71(2 \mathrm{H}, \mathrm{d}, J=8.3 \mathrm{~Hz}), 7.35-7.30(2 \mathrm{H}, \mathrm{m}), 7.30-7.24(8 \mathrm{H}, \mathrm{m}), 7.19-7.15$ (2H, m), $7.00(1 \mathrm{H}, \mathrm{dt}, J=15.0,7.2 \mathrm{~Hz}), 6.34(1 \mathrm{H}, \mathrm{dt}, J=15.0,1.3 \mathrm{~Hz}), 4.53(1 \mathrm{H}, \mathrm{d}, J=11.2 \mathrm{~Hz}), 4.47$ $(2 \mathrm{H}, \mathrm{s}), 4.40(1 \mathrm{H}, \mathrm{d}, J=11.2 \mathrm{~Hz}), 3.92(1 \mathrm{H}, \mathrm{ddd}, J=5.3,4.9,4.6 \mathrm{~Hz}), 3.66(1 \mathrm{H}, \mathrm{dt}, J=6.8,4.9 \mathrm{~Hz}), 3.50$ $(1 \mathrm{H}, \mathrm{dd}, J=9.7,4.6 \mathrm{~Hz}), 3.46(1 \mathrm{H}, \mathrm{dd}, J=9.7,5.3 \mathrm{~Hz}), 2.51-2.47(2 \mathrm{H}, \mathrm{m}), 2.40(3 \mathrm{H}, \mathrm{s}), 0.95(3 \mathrm{H}, \mathrm{d}, J$ $=6.8 \mathrm{~Hz}), 0.94(3 \mathrm{H}, \mathrm{d}, J=6.8 \mathrm{~Hz}), 0.922(3 \mathrm{H}, \mathrm{t}, J=8.1 \mathrm{~Hz}), 0.919(3 \mathrm{H}, \mathrm{t}, J=7.9 \mathrm{~Hz}), 0.91(1 \mathrm{H}$, septet, $J=6.8 \mathrm{~Hz}), 0.64-0.53(4 \mathrm{H}, \mathrm{m}) ;{ }^{13} \mathrm{C} \mathrm{NMR}\left(150 \mathrm{MHz}, \mathrm{CDCl}_{3}\right) \delta 144.1,143.7,137.91,137.89,137.7$, $132.3,129.8,128.34,128.29,127.9,127.8,127.7,127.6(\times 2), 78.7,73.3,72.7,72.6,71.4,32.8,21.6$, 17.29, 17.27, 12.9, 7.04, 7.02, 3.8, 3.7; HRFABMS $m / z$ calcd for $\mathrm{C}_{34} \mathrm{H}_{46} \mathrm{O}_{5} \mathrm{SSiNa}\left(\mathrm{MNa}^{+}\right) 617.2733$, found 617.2756.

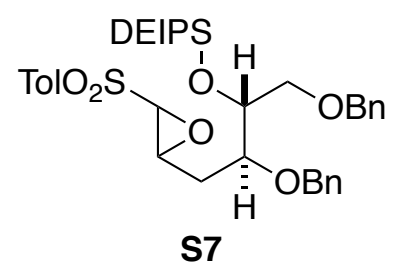

Epoxy sulfone S7. To a solution of $t$-BuOK $(2.05 \mathrm{~g}, 18.3 \mathrm{mmol})$ in $\mathrm{THF}(20 \mathrm{~mL})$ at $-80{ }^{\circ} \mathrm{C}$ were added $t$ - $\mathrm{BuOOH}(3.83 \mathrm{~mL}$ of a $5.5 \mathrm{M}$ solution in nonane, $21.1 \mathrm{mmol})$ and a solution of vinyl sulfone $\mathbf{S 6}(8.36 \mathrm{~g}$, 
$14.1 \mathrm{mmol})$ in THF $(70 \mathrm{~mL})$. The reaction mixture was stirred at $-80{ }^{\circ} \mathrm{C}$ for $15 \mathrm{~min}$ and then warmed slowly to room temperature. After stirring at room temperature for $2 \mathrm{~h}$, the reaction was quenched with saturated aqueous $\mathrm{NH}_{4} \mathrm{Cl}$ solution. The reaction mixture was extracted with EtOAc, and the extract was washed with saturated aqueous $\mathrm{Na}_{2} \mathrm{~S}_{2} \mathrm{O}_{3}$ solution, water, and brine, dried, and concentrated under reduced pressure. Purification by flash chromatography (15\% EtOAc in hexane) afforded epoxy sulfone S7 (6.87 g, 80\%, 58:42 mixture of diastereomers by $\left.{ }^{1} \mathrm{H} \mathrm{NMR}\right)$ as a colorless oil. $[\alpha]^{25} \mathrm{D}-10.9\left(c 1.25, \mathrm{CHCl}_{3}\right) ; \mathrm{IR}$ (film) 2952, 2914, 2873, 1372, 1153, $1089 \mathrm{~cm}^{-1} ;{ }^{1} \mathrm{H}$ NMR for the major isomer (500 MHz, $\left.\mathrm{CDCl}_{3}\right) \delta$ 7.76-7.74 (2H, m), 7.36-7.27 (12H, m), 4.61 and $4.50($ each $1 \mathrm{H}, \mathrm{d}, J=11.3 \mathrm{~Hz}), 4.48(2 \mathrm{H}, \mathrm{s}), 3.98(1 \mathrm{H}$, m), $3.88(1 \mathrm{H}, \mathrm{d}, J=1.7 \mathrm{~Hz}), 3.75-3.69(2 \mathrm{H}, \mathrm{m}), 3.51-3.42(2 \mathrm{H}, \mathrm{m}), 2.45(3 \mathrm{H}, \mathrm{s}), 2.01(1 \mathrm{H}, \mathrm{ddd}, J=14.7$, 8.5, $5.7 \mathrm{~Hz}), 1.84(1 \mathrm{H}, \mathrm{ddd}, J=14.7,5.5,4.0 \mathrm{~Hz}), 0.97-0.87(13 \mathrm{H}, \mathrm{m}), 0.63-0.55(4 \mathrm{H}, \mathrm{m}) ;{ }^{1} \mathrm{H}$ NMR for the minor isomer $\left(500 \mathrm{MHz}, \mathrm{CDCl}_{3}\right) \delta 7.79-7.78(2 \mathrm{H}, \mathrm{m}), 7.36-7.27(12 \mathrm{H}, \mathrm{m}), 4.64$ and $4.56($ each $1 \mathrm{H}$, d, $J=11.0 \mathrm{~Hz}), 4.47(2 \mathrm{H}, \mathrm{s}), 3.98(1 \mathrm{H}, \mathrm{m}), 3.92(1 \mathrm{H}, \mathrm{d}, J=1.7 \mathrm{~Hz}), 3.75-3.69(2 \mathrm{H}, \mathrm{m}), 3.51-3.42(2 \mathrm{H}$ m), $2.45(3 \mathrm{H}, \mathrm{s}), 1.99$ (1H, ddd, $J=14.7,9.6,4.9 \mathrm{~Hz}), 1.69(1 \mathrm{H}, \mathrm{ddd}, J=14.7,7.4,2.8 \mathrm{~Hz}), 0.97-0.87$ $(13 \mathrm{H}, \mathrm{m}), 0.63-0.55(4 \mathrm{H}, \mathrm{m}) ;{ }^{13} \mathrm{C} \mathrm{NMR}$ for the major isomer $\left(125 \mathrm{MHz}, \mathrm{CDCl}_{3}\right) \delta 145.4,138.0,137.9$ $134.0,129.9,128.8,128.4,128.3,127.9,127.8,127.7,127.6,77.9,73.4,72.6,72.5,71.4,68.3,56.2,31.5$ $21.7,17.3(\times 2), 12.9,7.04,7.02,3.8,3.7 ;{ }^{13} \mathrm{C} \mathrm{NMR}$ for the minor isomer $\left(125 \mathrm{MHz}, \mathrm{CDCl}_{3}\right) \delta 145.4$ $138.1,138.0,134.0,129.9,128.8,128.34,128.32,128.1,127.7,127.65,127.61,77.6,73.3,72.8,72.7$ 71.4, 69.0, 56.1, 32.0, 21.7, $17.3(\times 2), 12.9,7.04,7.02,3.8,3.7$; HRFABMS $m / z$ calcd for $\mathrm{C}_{34} \mathrm{H}_{46} \mathrm{O}_{6} \mathrm{SSiNa}_{\mathrm{N}}$ $\left(\mathrm{MNa}^{+}\right)$633.2682, found 633.2689.

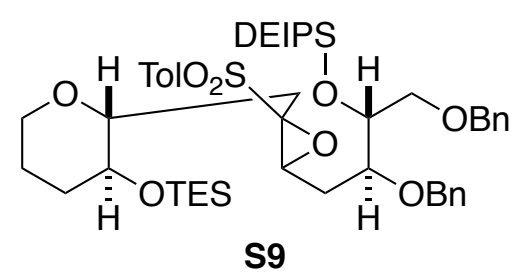

Coupling product S9. To a solution of ((2R,3S)-3-((triethylsilyl)oxy)tetrahydro-2H-pyran-2-yl)methyl trifluoromethanesulfonate $(\mathbf{S 8})^{4}(4.22 \mathrm{~g}, 11.1 \mathrm{mmol})$ and epoxy sulfone $\mathbf{S} 7(10.2 \mathrm{~g}, 16.7 \mathrm{mmol}, \mathrm{dr}=58: 42)$ 
in THF $(84 \mathrm{~mL})$ and HMPA $(3.80 \mathrm{~mL}, 33.4 \mathrm{mmol})$ at $-100{ }^{\circ} \mathrm{C}$ was added $n$-BuLi $(10.4 \mathrm{~mL}$ of a $1.60 \mathrm{M}$ solution in hexane, $16.7 \mathrm{mmol}$ ), and the reaction mixture was stirred at $-100{ }^{\circ} \mathrm{C}$ for $30 \mathrm{~min}$. The reaction was quenched with saturated aqueous $\mathrm{NH}_{4} \mathrm{Cl}$ solution. The reaction mixture was warmed to room temperature and extracted with EtOAc. The extract was washed with water and brine, dried, and concentrated under reduced pressure. Flash chromatography (10\% Acetone in $n$-hexane) afforded the coupling product S9 (7.80 g, 84\%, 70:30 mixture of diastereomers by ${ }^{1} \mathrm{H}$ NMR) and unreacted epoxy sulfone S7 (3.86 g, 86\% recovered based on consumed $\mathbf{S 7}$, dr $=34: 66$ ). A small portion of the diasteremeric mixture was separated for analytical purpose by flash chromatography (12\% EtOAc in $n$ hexane).

Major diastereomer of S9. Colorless oil; $[\alpha]^{28} \mathrm{D}+15.4\left(c 1.11, \mathrm{CHCl}_{3}\right)$; IR (film) 2953, 2875, 1455, 1149 , $1098 \mathrm{~cm}^{-1} ;{ }^{1} \mathrm{H} \mathrm{NMR}\left(600 \mathrm{MHz}, \mathrm{CDCl}_{3}\right) \delta 7.80(2 \mathrm{H}, \mathrm{d}, J=8.3 \mathrm{~Hz}), 7.35-7.24(12 \mathrm{H}, \mathrm{m}), 4.62$ and 4.55 (each 1H, d, $J=11.4 \mathrm{~Hz}), 4.50$ and $4.46($ each $1 \mathrm{H}, \mathrm{d}, J=11.9 \mathrm{~Hz}), 3.96(1 \mathrm{H}, \mathrm{td}, J=5.1,4.2 \mathrm{~Hz}), 3.75$ $(1 \mathrm{H}, \mathrm{t}, J=6.0 \mathrm{~Hz}), 3.69(1 \mathrm{H}, \mathrm{ddd}, J=7.2,5.2,4.2 \mathrm{~Hz}), 3.60(1 \mathrm{H}, \mathrm{ddt}, J=11.3,4.4,1.5 \mathrm{~Hz}), 3.51(1 \mathrm{H}$, dd, $J=9.9,5.1 \mathrm{~Hz}), 3.49(1 \mathrm{H}, \mathrm{dd}, J=9.9,5.1 \mathrm{~Hz}), 3.13(1 \mathrm{H}, \mathrm{ddd}, J=10.3,8.6,4.4 \mathrm{~Hz}), 3.05(1 \mathrm{H}, \mathrm{ddd}$, $J=9.9,8.6,1.7 \mathrm{~Hz}), 2.94(1 \mathrm{H}, \mathrm{dd}, J=15.6,1.7 \mathrm{~Hz}), 2.86(1 \mathrm{H}, \mathrm{td}, J=11.6,2.8 \mathrm{~Hz}), 2.41(3 \mathrm{H}, \mathrm{s}), 1.97$ $(1 \mathrm{H}, \mathrm{ddd}, J=15.5,7.2,6.0 \mathrm{~Hz}), 1.96(1 \mathrm{H}, \mathrm{ddd}, 15.5,6.0,5.2 \mathrm{~Hz}), 1.92(1 \mathrm{H}, \mathrm{m}), 1.77(1 \mathrm{H}, \mathrm{dd}, J=15.6$ $9.9 \mathrm{~Hz}), 1.57-1.45(2 \mathrm{H}, \mathrm{m}), 1.26(1 \mathrm{H}, \mathrm{tdd}, J=12.7,10.3,4.8 \mathrm{~Hz}), 0.99-0.90(22 \mathrm{H}, \mathrm{m}), 0.64-0.54(10 \mathrm{H}$, $\mathrm{m}) ;{ }^{13} \mathrm{C} \mathrm{NMR}\left(150 \mathrm{MHz}, \mathrm{CDCl}_{3}\right) \delta 144.6,138.3,138.1,134.1,129.6,129.3,128.30,128.27,127.8,127.7$, $127.6,127.5,78.7,78.4,75.0,73.3,73.2,72.6,71.7,71.3,67.0,58.3,33.4,29.1,28.3,25.3,21.6,17.35$ 17.33, 12.9, 7.08, 7.06, 6.9, 5.1, 3.8, 3.7; HRFABMS $m / z$ calcd for $\mathrm{C}_{46} \mathrm{H}_{70} \mathrm{O}_{8} \mathrm{SSi}_{2} \mathrm{Na}\left(\mathrm{MNa}^{+}\right) 861.4228$, found 861.4240 .

Minor diastereomer of S9. Colorless oil; $[\alpha]^{28}$-43.7 (c 1.18, $\mathrm{CHCl}_{3}$ ); IR (film) 2953, 2875, 1455, 1149, $1098 \mathrm{~cm}^{-1}$; ${ }^{1} \mathrm{H}$ NMR $\left(500 \mathrm{MHz}, \mathrm{CDCl}_{3}\right) \delta 7.80(2 \mathrm{H}, \mathrm{d}, J=8.2 \mathrm{~Hz}), 7.36-7.23(12 \mathrm{H}, \mathrm{m}), 4.63$ and $4.55($ each $1 \mathrm{H}, \mathrm{d}, J=11.1 \mathrm{~Hz}), 4.51$ and $4.48($ each $1 \mathrm{H}, \mathrm{d}, J=11.9 \mathrm{~Hz}), 4.06(1 \mathrm{H}, \mathrm{dd}, J=9.6,2.3 \mathrm{~Hz})$, $3.92(1 \mathrm{H}, \mathrm{ddd}, J=4.8,4.7,4.5 \mathrm{~Hz}), 3.73(1 \mathrm{H}, \mathrm{ddd}, J=10.3,4.7,3.1 \mathrm{~Hz}), 3.63(1 \mathrm{H}, \mathrm{ddd}, J=11.3,8.8$, 
$1.8 \mathrm{~Hz}), 3.62(1 \mathrm{H}, \mathrm{m}), 3.53(1 \mathrm{H}, \mathrm{dd}, J=9.9,4.8 \mathrm{~Hz}), 3.52(1 \mathrm{H}, \mathrm{dd}, J=9.9,4.5 \mathrm{~Hz}), 3.20(1 \mathrm{H}, \mathrm{td}, J=11.2$, $3.1 \mathrm{~Hz}), 3.10(1 \mathrm{H}, \mathrm{ddd}, J=10.1,8.9,4.5 \mathrm{~Hz}), 2.60(1 \mathrm{H}, \mathrm{dd}, J=15.3,1.8 \mathrm{~Hz}), 2.42(3 \mathrm{H}, \mathrm{s}), 2.17(1 \mathrm{H}$, ddd, $J=15.2,10.3,2.3 \mathrm{~Hz}), 1.94(1 \mathrm{H}, \mathrm{m}), 1.61(1 \mathrm{H}, \mathrm{ddd}, J=15.2,9.6,3.1 \mathrm{~Hz}), 1.48(1 \mathrm{H}, \mathrm{dd}, J=15.3,11.3$ $\mathrm{Hz}), 1.57-1.38(3 \mathrm{H}, \mathrm{m}), 0.98-0.92(13 \mathrm{H}, \mathrm{m}), 0.88(9 \mathrm{H}, \mathrm{t}, J=7.9 \mathrm{~Hz}), 0.67-0.48(10 \mathrm{H}, \mathrm{m}) ;{ }^{13} \mathrm{C} \mathrm{NMR}$ $\left(125 \mathrm{MHz}, \mathrm{CDCl}_{3}\right) \delta 144.7,138.3,138.2,133.1,129.7,129.4,128.32,128.27,128.1,127.7,127.53$ $127.49,78.4,78.3,74.4,73.9,73.35,73.34,71.78,71.76,67.2,59.3,33.5,31.0,30.7,25.5,21.6,17.3$ $(\times 2), 12.9,7.12,7.06,6.8,5.1,3.9,3.7 ; \mathrm{HRFABMS} m / z$ calcd for $\mathrm{C}_{46} \mathrm{H}_{70} \mathrm{O}_{8} \mathrm{SSi}_{2} \mathrm{Na}\left(\mathrm{MNa}^{+}\right) 861.4228$ found 861.4210 .

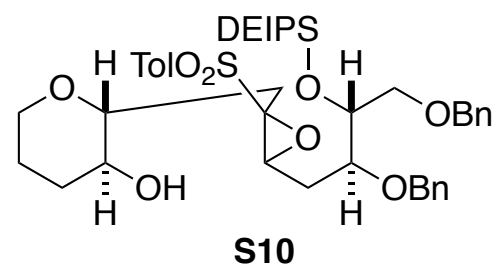

Alcohol S10. To a solution of the coupling product S9 (70:30 diastereomeric mixture, $9.30 \mathrm{~g}, 11.1$ $\mathrm{mmol})$ in $\mathrm{CH}_{2} \mathrm{Cl}_{2}(111 \mathrm{~mL})$ and $\mathrm{MeOH}(111 \mathrm{~mL})$ was added pyridinium $p$-toluenesulfonate $(557 \mathrm{mg}, 2.22$ $\mathrm{mmol}$ ), and the reaction mixture was stirred at room temperature for $10 \mathrm{~min}$. The reaction was quenched with $\mathrm{Et}_{3} \mathrm{~N}(5 \mathrm{~mL})$, and the reaction mixture was concentrated under reduced pressure. Alcohol S10 (5.23 g) and the remaining starting TES ether S9 (3.06 g) were separated by flash chromatography (30\% EtOAc in hexane). The recovered TES ether S9 was subjected to the same protocol twice to afford additional alcohol S10 (2.04 g). In total, $7.27 \mathrm{~g}$ (71:29 diastereomeric mixture, 90\%) of S10 was obtained as a colorless oil. $[\alpha]^{27} \mathrm{D}+12.7$ (c 1.06, $\mathrm{CHCl}_{3}$ ); IR (film) 3445, 2939, 2864, 1455, 1147, $1093 \mathrm{~cm}^{-1}$; ${ }^{1} \mathrm{H}$ NMR for the major isomer $\left(600 \mathrm{MHz}, \mathrm{CDCl}_{3}\right) \delta 7.76(2 \mathrm{H}, \mathrm{d}, J=8.3 \mathrm{~Hz}), 7.35-7.25(12 \mathrm{H}, \mathrm{m}), 4.61$ and 4.50 (each $1 \mathrm{H}, \mathrm{d}, J=11.6 \mathrm{~Hz}), 4.49$ and $4.46($ each $1 \mathrm{H}, \mathrm{d}, J=11.9 \mathrm{~Hz}), 3.98-3.94(1 \mathrm{H}, \mathrm{m}), 3.74-3.70(2 \mathrm{H}$, m), $3.62(1 \mathrm{H}, \mathrm{t}, J=6.1 \mathrm{~Hz}), 3.52-3.46(2 \mathrm{H}, \mathrm{m}), 3.34(1 \mathrm{H}, \mathrm{ddd}, J=9.2,6.7,4.6 \mathrm{~Hz}), 3.16-3.09(2 \mathrm{H}, \mathrm{m})$, $2.61(1 \mathrm{H}, \mathrm{dd}, J=15.8,4.6 \mathrm{~Hz}), 2.41(3 \mathrm{H}, \mathrm{s}), 2.09-2.02(1 \mathrm{H}, \mathrm{m}), 1.992(1 \mathrm{H}, \mathrm{dd}, J=15.8,6.6 \mathrm{~Hz}), 1.991$ $(1 \mathrm{H}, \mathrm{ddd}, J=14.9,8.6,6.1 \mathrm{~Hz}), 1.92(1 \mathrm{H}, \mathrm{ddd}, J=14.9,6.2,3.9 \mathrm{~Hz}), 1.62(1 \mathrm{H}, \mathrm{br} \mathrm{s}), 1.59-1.54(2 \mathrm{H}, \mathrm{m})$, 
1.34-1.22 (1H, m), 0.98-0.89 (13H, m), 0.64-0.57 (4H, m); ${ }^{1} \mathrm{H}$ NMR for the minor isomer $(600 \mathrm{MHz}$,

$\left.\mathrm{CDCl}_{3}\right) \delta 7.80(2 \mathrm{H}, \mathrm{d}, J=8.3 \mathrm{~Hz}), 7.35-7.25(12 \mathrm{H}, \mathrm{m}), 4.63$ and $4.50($ each $1 \mathrm{H}, \mathrm{d}, J=11.6 \mathrm{~Hz}), 4.48(2 \mathrm{H}$, s), 3.98-3.94 (1H, m), $3.93(1 \mathrm{H}, \mathrm{dd}, J=9.0,3.1 \mathrm{~Hz}), 3.74-3.70(1 \mathrm{H}, \mathrm{m}), 3.64(1 \mathrm{H}, \mathrm{m}), 3.52-3.46(3 \mathrm{H}$, m), 3.16-3.09 (2H, m), $2.66(1 \mathrm{H}, \mathrm{dd}, J=15.8,2.4 \mathrm{~Hz}), 2.42(3 \mathrm{H}, \mathrm{s}), 2.16(1 \mathrm{H}, \mathrm{ddd}, J=15.2,10.1,3.1$ Hz), 2.09-2.02 (1H, m), $1.76(1 \mathrm{H}, \mathrm{dd}, J=15.8,8.6 \mathrm{~Hz}), 1.67(1 \mathrm{H}, \mathrm{ddd}, J=15.2,9.0,2.9 \mathrm{~Hz}), 1.62(1 \mathrm{H}$, br s), 1.59-1.54 (2H, m), 1.34-1.22 (1H, m), 0.98-0.89 (13H, m), 0.64-0.57 (4H, m); ${ }^{13} \mathrm{C}$ NMR for the major isomer $\left(150 \mathrm{MHz}, \mathrm{CDCl}_{3}\right) \delta 145.2,138.3,138.0,132.5,129.8,129.5,128.35,128.29,127.80$, $127.76,127.7,127.5,78.7,78.2,74.9,73.4,72.7,72.5,71.6,70.6,67.4,59.1,33.3,29.3,28.8,25.5,21.7$, $17.32,17.30,12.9,7.1,7.0,3.8,3.7 ;{ }^{13} \mathrm{C} \mathrm{NMR}$ for the minor isomer $\left(150 \mathrm{MHz}, \mathrm{CDCl}_{3}\right) \delta 145.1,138.2$, $138.1,132.4,129.9,129.5,128.3,128.1,127.8,127.72,127.65,127.5,78.8,78.3,74.9,73.43,73.35,73.1$, 71.7, 70.5, 67.3, 59.7, 33.3, 30.4, 28.8, 25.5, 21.7, 17.32, 17.30, 12.9, 7.09, 7.06, 3.8, 3.7; HRFABMS $m / z$ calcd for $\mathrm{C}_{40} \mathrm{H}_{56} \mathrm{O}_{8} \mathrm{SSiNa}\left(\mathrm{MNa}^{+}\right)$747.3363, found 747.3378.

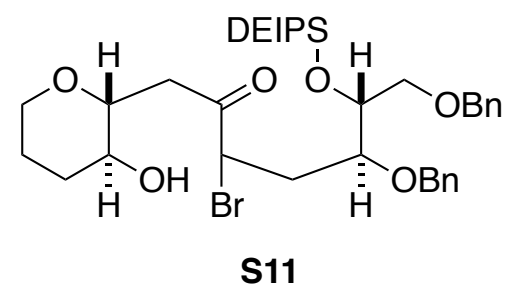

Bromoketone S11. To a solution of S10 (71:29 diastereomeric mixture, $7.43 \mathrm{~g}, 10.3 \mathrm{mmol})$ and $\mathrm{LiBr}$ (1.25 g, $14.4 \mathrm{mmol})$ in $\mathrm{CH}_{2} \mathrm{Cl}_{2}(100 \mathrm{~mL})$ at $-15{ }^{\circ} \mathrm{C}$ was added $\mathrm{MgBr}_{2} \cdot \mathrm{OEt}_{2}(3.71 \mathrm{~g}, 14.4 \mathrm{mmol})$, and the mixture was stirred at $0{ }^{\circ} \mathrm{C}$ for $3.5 \mathrm{~h}$. The reaction was quenched with saturated aqueous $\mathrm{NaHCO}_{3}$ solution, and the reaction mixture was extracted with EtOAc. The extract was washed with water, and brine, dried, and concentrated under reduced pressure. Purification by flash chromatography (30\% EtOAc in hexane) afforded bromoketone $\mathbf{S 1 1}$ (67:33 diastereomeric mixture, $5.72 \mathrm{~g}, 86 \%)$ as a colorless oil. $[\alpha]^{28} \mathrm{D}-32.0(c$ 1.11, $\mathrm{CHCl}_{3}$ ); IR (film) 3448, 2940, 2864, 1718, $1094 \mathrm{~cm}^{-1} ;{ }^{1} \mathrm{H}$ NMR for the major isomer $(600 \mathrm{MHz}$, $\left.\mathrm{CDCl}_{3}\right) \delta 7.35-7.25(10 \mathrm{H}, \mathrm{m}), 4.76$ and $4.51($ each $1 \mathrm{H}, \mathrm{d}, J=11.2 \mathrm{~Hz}), 4.62(1 \mathrm{H}, \mathrm{dd}, J=10.6,3.5 \mathrm{~Hz})$, $4.50(\mathrm{~s}, 2 \mathrm{H}), 4.06(1 \mathrm{H}, \mathrm{td}, J=5.5,2.8 \mathrm{~Hz}), 3.87(1 \mathrm{H}, \mathrm{dt}, J=10.3,2.8 \mathrm{~Hz}), 3.97(1 \mathrm{H}, \mathrm{ddt}, J=11.2,3.8$, 
$1.9 \mathrm{~Hz}), 3.50(1 \mathrm{H}, \mathrm{dd}, J=9.7,5.5 \mathrm{~Hz}), 3.49(1 \mathrm{H}, \mathrm{dd}, J=9.7,5.5 \mathrm{~Hz}), 3.45(1 \mathrm{H}, \mathrm{ddd}, J=9.1,7.1,4.8 \mathrm{~Hz})$, 3.27-3.17 (2H, m), $3.08(1 \mathrm{H}, \mathrm{dd}, J=15.8,4.8 \mathrm{~Hz}), 2.85(1 \mathrm{H}, \mathrm{dd}, J=15.8,7.1 \mathrm{~Hz}), 2.25(1 \mathrm{H}, \mathrm{ddd}, J=$ $15.4,10.2,3.6 \mathrm{~Hz}), 2.06-2.01(1 \mathrm{H}, \mathrm{m}), 2.03(1 \mathrm{H}, \mathrm{ddd}, J=15.4,10.6,2.8 \mathrm{~Hz}), 1.91(1 \mathrm{H}$, br s), $1.64-1.58$ $(2 \mathrm{H}, \mathrm{m}), 1.38-1.26(1 \mathrm{H}, \mathrm{m}), 0.99-0.94(13 \mathrm{H}, \mathrm{m}), 0.67-0.59(4 \mathrm{H}, \mathrm{m}) ;{ }^{1} \mathrm{H}$ NMR for the minor isomer $(600$ $\left.\mathrm{MHz}, \mathrm{CDCl}_{3}\right) \delta 7.35-7.25(10 \mathrm{H}, \mathrm{m}), 4.62(1 \mathrm{H}, \mathrm{dd}, J=9.7,4.2 \mathrm{~Hz}), 4.60$ and $4.34($ each $1 \mathrm{H}, \mathrm{d}, J=11.2$ $\mathrm{Hz}), 4.52$ and 4.49 (each 1H, d, $J=11.9 \mathrm{~Hz}), 4.02(1 \mathrm{H}, \mathrm{td}, J=5.7,2.9 \mathrm{~Hz}), 3.72(1 \mathrm{H}, \mathrm{ddt}, J=11.0,3.8$, $2.1 \mathrm{~Hz}), 3.59(1 \mathrm{H}, \mathrm{dt}, J=8.8,3.1 \mathrm{~Hz}), 3.54(1 \mathrm{H}, \mathrm{dd}, J=9.7,5.7 \mathrm{~Hz}), 3.49(1 \mathrm{H}, \mathrm{dd}, J=9.7,5.7 \mathrm{~Hz}), 3.37$ (1H, ddd, $J=9.1,7.7,4.1 \mathrm{~Hz}), 3.27-3.17(2 \mathrm{H}, \mathrm{m}), 3.02(1 \mathrm{H}, \mathrm{dd}, J=15.7,4.1 \mathrm{~Hz}), 2.71(1 \mathrm{H}, \mathrm{dd}, J=15.7$, $7.7 \mathrm{~Hz}), 2.45(1 \mathrm{H}, \mathrm{ddd}, J=14.6,9.8,3.3 \mathrm{~Hz}), 2.29(1 \mathrm{H}, \mathrm{ddd}, J=14.6,8.8,4.2 \mathrm{~Hz}), 2.06-2.01(1 \mathrm{H}, \mathrm{m})$, $1.82\left(1 \mathrm{H}\right.$, br s), $1.64-1.58(2 \mathrm{H}, \mathrm{m}), 1.38-1.26(1 \mathrm{H}, \mathrm{m}), 0.99-0.94(13 \mathrm{H}, \mathrm{m}), 0.67-0.59(4 \mathrm{H}, \mathrm{m}) ;{ }^{13} \mathrm{C} \mathrm{NMR}$ for the major isomer $\left(150 \mathrm{MHz}, \mathrm{CDCl}_{3}\right) \delta 202.4,138.4,138.0,128.3(\times 2), 128.0,127.7,127.60,127.58$, $78.8,78.5,73.4,73.0(\times 2), 71.7,70.3,67.7,52.3,43.0,34.0,33.0,25.4,17.3(\times 2), 12.9,7.1(\times 2), 3.8,3.7$; ${ }^{13} \mathrm{C} \mathrm{NMR}$ for the minor isomer $\left(150 \mathrm{MHz}, \mathrm{CDCl}_{3}\right) \delta 202.6,138.3,138.1,128.29,128.25,128.0,127.7$, $127.6,127.5,79.3,79.0,73.4,72.8,72.4,71.8,70.3,67.4,51.1,43.1,34.8,32.9,25.5,17.3(\times 2), 12.9$, $7.1(\times 2), 3.8,3.7$; HRFABMS $m / z$ calcd for $\mathrm{C}_{33} \mathrm{H}_{49} \mathrm{O}_{6} \mathrm{BrSiNa}\left(\mathrm{MNa}^{+}\right) 671.2379$, found 671.2372.

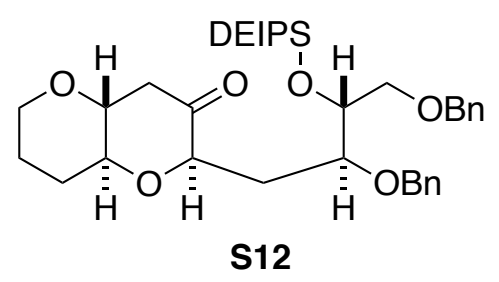

Six-membered ketone S12. To a solution of bromoketone $\mathbf{S 1 1}$ (67:33 diastereomeric mixture, $5.72 \mathrm{~g}$, $8.80 \mathrm{mmol})$ in $\mathrm{CH}_{2} \mathrm{Cl}_{2}(59 \mathrm{~mL})$ was added DBU $(1.36 \mathrm{~mL}, 9.68 \mathrm{mmol})$ at $0{ }^{\circ} \mathrm{C}$, and the reaction mixture was stirred at $0{ }^{\circ} \mathrm{C}$ for $2.5 \mathrm{~h}$. The reaction was quenched with saturated aqueous $\mathrm{NH}_{4} \mathrm{Cl}$ solution, and the mixture was extracted with EtOAc-hexane (1:1). The extract was washed with brine, dried, and concentrated under reduced pressure. Purification by flash chromatography (20\% EtOAc in hexane) afforded the cyclic ketone $\mathbf{S 1 2}$ (single diastereomer, $4.40 \mathrm{~g}, 88 \%)$ as a colorless oil. $[\alpha]^{27} \mathrm{D}-19.4(c 1.21$, 
$\mathrm{CHCl}_{3}$ ); IR (film) 2944, 2865, 1726, $1097 \mathrm{~cm}^{-1} ;{ }^{1} \mathrm{H} \mathrm{NMR}\left(600 \mathrm{MHz}, \mathrm{CDCl}_{3}\right) \delta$ 7.35-7.25 (10H, m), 4.68

and 4.43 (each 1H, d, $J=11.7 \mathrm{~Hz}), 4.53$ and $4.50($ each $1 \mathrm{H}, \mathrm{d}, J=11.9 \mathrm{~Hz}), 4.03(1 \mathrm{H}, \mathrm{td}, J=5.3,3.2 \mathrm{~Hz})$, $3.89(1 \mathrm{H}, \mathrm{m}), 3.80(1 \mathrm{H}, \mathrm{dd}, J=6.6,5.1 \mathrm{~Hz}), 3.78(1 \mathrm{H}, \mathrm{ddd}, J=8.5,4.4,3.2 \mathrm{~Hz}), 3.59(1 \mathrm{H}, \mathrm{dd}, J=9.8$, $5.0 \mathrm{~Hz}), 3.50(1 \mathrm{H}, \mathrm{dd}, J=9.8,5.5 \mathrm{~Hz}), 3.35(1 \mathrm{H}, \mathrm{m}), 3.22(1 \mathrm{H}, \mathrm{ddd}, J=10.8,9.2,4.4 \mathrm{~Hz}), 3.17(1 \mathrm{H}$, ddd, $J=11.4,9.2,5.5 \mathrm{~Hz}), 2.72(1 \mathrm{H}, \mathrm{dd}, J=15.4,5.5 \mathrm{~Hz}), 2.25(1 \mathrm{H}, \mathrm{dd}, J=15.4,11.4 \mathrm{~Hz}), 2.14(1 \mathrm{H}, \mathrm{m}), 2.07$ (1H, ddd, $J=14.6,6.6,4.4 \mathrm{~Hz}), 1.94(1 \mathrm{H}, \mathrm{ddd}, J=14.6,8.5,5.1 \mathrm{~Hz}), 1.77-1.70(2 \mathrm{H}, \mathrm{m}), 1.47(1 \mathrm{H}, \mathrm{dtd}$, $J=12.3,10.8,7.2 \mathrm{~Hz}), 1.02-0.92(13 \mathrm{H}, \mathrm{m}), 0.69-0.59(4 \mathrm{H}, \mathrm{m}) ;{ }^{13} \mathrm{C} \mathrm{NMR}\left(150 \mathrm{MHz}, \mathrm{CDCl}_{3}\right) \delta 205.4$, $138.7,138.3,128.2(\times 2), 127.9,127.7,127.45,127.42,80.4,77.6,77.3,76.8,73.5,73.4,72.14,72.05$, 67.4, 45.2, 30.7, 29.2, 25.1, $17.3(\times 2), 13.0,7.1(\times 2), 3.81,3.75 ;$ HRFABMS $m / z$ calcd for $\mathrm{C}_{33} \mathrm{H}_{48} \mathrm{O}_{6} \mathrm{SiNa}$ $\left(\mathrm{MNa}^{+}\right)$591.3118, found 591.3096.

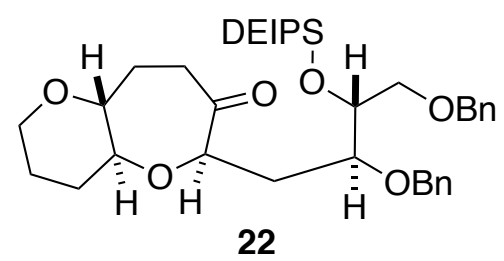

Seven-membered ketone 22. To a suspension of the six-membered ketone $\mathbf{S 1 2}$ (4.40 g, $7.74 \mathrm{mmol})$ and MS $4 \AA$ (22.0 g) in $\mathrm{CH}_{2} \mathrm{Cl}_{2}(52 \mathrm{~mL})$ at $-80{ }^{\circ} \mathrm{C}$ were added $\mathrm{BF}_{3} \cdot \mathrm{OEt}_{2}(1.05 \mathrm{~mL}, 8.51 \mathrm{mmol})$ and $\mathrm{TMSCH}_{2} \mathrm{~N}_{2}(11.6 \mathrm{~mL}$ of a $2.0 \mathrm{M}$ solution in hexane, $23.2 \mathrm{mmol})$, and the reaction mixture was stirred at $-80^{\circ} \mathrm{C}$ for $1 \mathrm{~h}$. The reaction was quenched with saturated aqueous $\mathrm{NaHCO}_{3}$ solution. MS $4 \AA$ was removed by filtration under reduced pressure, and the filtrate was extracted with EtOAc. The extract was washed with brine, dried, and concentrated under reduced pressure to give the crude product (5.33 g). A mixture of the crude product and PPTS $(2.05 \mathrm{~g}, 8.15 \mathrm{mmol})$ in $\mathrm{CH}_{2} \mathrm{Cl}_{2}(58 \mathrm{~mL})$ and $\mathrm{MeOH}(58 \mathrm{~mL})$ was stirred at room temperature for $1 \mathrm{~h}$. The reaction was quenched with $\mathrm{Et}_{3} \mathrm{~N}(5 \mathrm{~mL})$, and the reaction mixture was concentrated under reduced pressure. Purification by flash chromatography $(15 \rightarrow 20 \%$ EtOAc in hexane $)$ afforded ketone $22(2.70 \mathrm{~g}, 60 \%)$ as a colorless oil. $[\alpha]^{26} \mathrm{D}+35.3\left(c 1.00, \mathrm{CHCl}_{3}\right)$; IR (film) 2941, 2867, 1714, $1094 \mathrm{~cm}^{-1} ;{ }^{1} \mathrm{H} \mathrm{NMR}\left(600 \mathrm{MHz}, \mathrm{CDCl}_{3}\right) \delta 7.35-7.24(10 \mathrm{H}, \mathrm{m}), 4.68$ and $4.53(1 \mathrm{H}, \mathrm{d}, J=11.7 \mathrm{~Hz})$, 
$4.49(2 \mathrm{H}, \mathrm{s}), 4.01(1 \mathrm{H}, \mathrm{td}, J=5.3,3.3 \mathrm{~Hz}), 3.95(1 \mathrm{H}, \mathrm{t}, J=6.3 \mathrm{~Hz}), 3.86(1 \mathrm{H}, \mathrm{ddt}, J=11.2,4.3,1.6 \mathrm{~Hz})$,

$3.77(1 \mathrm{H}, \mathrm{ddd}, J=7.8,4.2,3.3 \mathrm{~Hz}), 3.53(1 \mathrm{H}, \mathrm{dd}, J=9.8,5.3 \mathrm{~Hz}), 3.49(1 \mathrm{H}, \mathrm{dd}, J=9.8,5.3 \mathrm{~Hz}), 3.33$ $(1 \mathrm{H}, \mathrm{td}, J=11.5,2.8 \mathrm{~Hz}), 3.18(1 \mathrm{H}, \mathrm{ddd}, J=10.9,9.0,4.3 \mathrm{~Hz}), 2.95(1 \mathrm{H}, \mathrm{ddd}, J=10.8,9.1,4.7 \mathrm{~Hz}), 2.78$ (1H, ddd, $J=13.8,12.7,2.3 \mathrm{~Hz}), 2.20(1 \mathrm{H}, \mathrm{ddd}, J=12.5,7.1,1.5 \mathrm{~Hz}), 2.09-2.02(2 \mathrm{H}, \mathrm{m}), 2.02(1 \mathrm{H}$, ddd, $J=14.5,8.3,5.9 \mathrm{~Hz}), 1.93(1 \mathrm{H}, \mathrm{ddd}, J=14.5,6.8,4.2 \mathrm{~Hz}), 1.70-1.61(2 \mathrm{H}, \mathrm{m}), 1.54-1.45$ (2H, m), 0.99$0.91(7 \mathrm{H}, \mathrm{m}), 0.95(3 \mathrm{H}, \mathrm{t}, J=8.0 \mathrm{~Hz}), 0.94(3 \mathrm{H}, \mathrm{t}, J=8.0 \mathrm{~Hz}), 0.66-0.59(4 \mathrm{H}, \mathrm{m}) ;{ }^{13} \mathrm{C} \mathrm{NMR}(150 \mathrm{MHz}$, $\left.\mathrm{CDCl}_{3}\right) \delta 215.5,138.7,138.2,128.3,128.2,127.7,127.50,127.48,127.3,84.1,81.9,81.4,77.4,73.5$, 73.3, 72.1, 71.9, 67.6, 36.5, 34.3, 31.1, 29.8, 25.7, 17.35, 17.33, 13.0, 7.09, 7.07, 3.82, 3.76; HRFABMS $m / z$ calcd for $\mathrm{C}_{34} \mathrm{H}_{50} \mathrm{O}_{6} \mathrm{SiNa}\left(\mathrm{MNa}^{+}\right)$605.3274, found 605.3270.

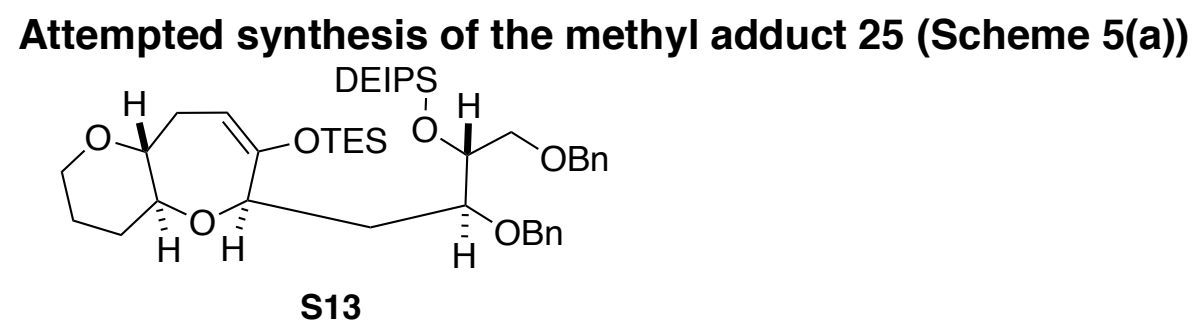

Silyl enol ether S13. To a solution of ketone $22(418 \mathrm{mg}, 0.718 \mathrm{mmol})$ in $\mathrm{THF}(18 \mathrm{~mL})$ at $-78{ }^{\circ} \mathrm{C}$ were added $\mathrm{Et}_{3} \mathrm{~N}(0.500 \mathrm{~mL}, 3.59 \mathrm{mmol})$, TESCl $(0.602 \mathrm{~mL}, 3.59 \mathrm{mmol})$, and LiHMDS (2.15 $\mathrm{mL}$ of a $1.0 \mathrm{M}$ solution in THF, $2.15 \mathrm{mmol}$ ). The reaction mixture was stirred at $-78^{\circ} \mathrm{C}$ for $1 \mathrm{~h}$. The reaction was quenched with phosphate buffer $(\mathrm{pH} 7)$ and the reaction mixture was extracted with EtOAc. The extract was washed with brine, dried, and concentrated under reduced pressure. Purification by flash chromatography (8\% EtOAc in $n$-hexane) afforded TES enol ether $\mathbf{S 1 3}$ (483 $\mathrm{mg}, 97 \%$ ) as a colorless oil. $[\alpha]^{25} \mathrm{D}-2.9$ (c 0.98, $\left.\mathrm{CHCl}_{3}\right)$; IR (film) 2953, 2875, 1651, 1118, $1096 \mathrm{~cm}^{-1} ;{ }^{1} \mathrm{H} \mathrm{NMR}\left(600 \mathrm{MHz}, \mathrm{CDCl}_{3}\right) \delta$ 7.32-7.23 (10H, m), $4.92(1 \mathrm{H}, \mathrm{ddd}, J=9.2,5.1,0.7 \mathrm{~Hz}), 4.61$ and $4.54($ each $1 \mathrm{H}, \mathrm{d}, J=11.4 \mathrm{~Hz}), 4.50$ $(2 \mathrm{H}, \mathrm{s}), 4.24(1 \mathrm{H}, \mathrm{ddd}, J=8.2,6.0,0.7 \mathrm{~Hz}), 3.98(1 \mathrm{H}, \mathrm{dt}, J=6.1,3.9 \mathrm{~Hz}), 3.84(1 \mathrm{H}, \mathrm{ddt}, J=11.0,4.0$, 1.6, $1.6 \mathrm{~Hz}), 3.65(1 \mathrm{H}, \mathrm{td}, J=6.1,4.0 \mathrm{~Hz}), 3.62(1 \mathrm{H}, \mathrm{dd}, J=9.9,3.9 \mathrm{~Hz}), 3.53(1 \mathrm{H}, \mathrm{dd}, J=9.9,6.1 \mathrm{~Hz})$, $3.31(1 \mathrm{H}, \mathrm{td}, J=11.0,3.9 \mathrm{~Hz}), 3.29(1 \mathrm{H}, \mathrm{ddd}, J=11.0,8.8,4.4 \mathrm{~Hz}), 2.93(1 \mathrm{H}, \mathrm{ddd}, J=10.0,8.8,3.1 \mathrm{~Hz})$, $2.34(1 \mathrm{H}, \mathrm{ddd}, J=15.0,10.0,5.1 \mathrm{~Hz}), 2.22(1 \mathrm{H}, \mathrm{ddd}, J=15.0,9.2,3.1 \mathrm{~Hz}), 2.04(1 \mathrm{H}, \mathrm{dt}, J=14.4,6.0$ 
Hz), $1.95(1 \mathrm{H}, \mathrm{m}), 1.83(1 \mathrm{H}, \mathrm{ddd}, J=14.4,8.2,6.2 \mathrm{~Hz}), 1.70-1.60(2 \mathrm{H}, \mathrm{m}), 1.44(1 \mathrm{H}, \mathrm{qd}, J=12.5,5.3$

$\mathrm{Hz}), 0.99-0.94(22 \mathrm{H}, \mathrm{m}), 0.69-0.61(10 \mathrm{H}, \mathrm{m}) ;{ }^{13} \mathrm{C} \mathrm{NMR}\left(150 \mathrm{MHz}, \mathrm{CDCl}_{3}\right) \delta 157.9,139.0,138.5,128.2$ (×2), 127.9, 127.5, 127.4, 127.3, 101.7, 84.3, 79.1, 78.6, 77.2, 74.8, 73.2, 72.5, 72.3, 67.9, 33.5, 31.1, 30.8, 26.2, 17.43, 17.38, 13.1, $7.2(\times 2), 6.7,4.9,3.94,3.86$; HRFABMS $m / z$ calcd for $\mathrm{C}_{40} \mathrm{H}_{64} \mathrm{O}_{6} \mathrm{Si}_{2} \mathrm{Na}_{(}\left(\mathrm{MNa}^{+}\right)$ 719.4139, found 719.4153 .

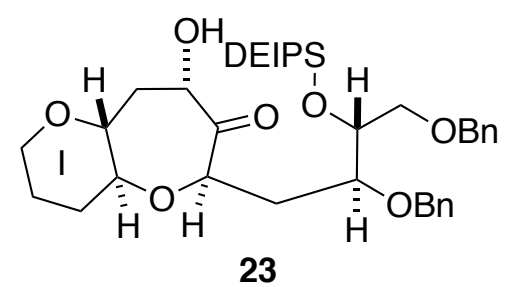

Hydroxy ketone 23. To a solution of TES enol ether S13 (480 mg, $0.705 \mathrm{mmol})$ and $N$ methylmorpholine $N$-oxide (NMO) $(826 \mathrm{mg}, 7.05 \mathrm{mmol})$ in THF $(20 \mathrm{~mL})$ and water $(5 \mathrm{~mL})$ was added $\mathrm{OsO}_{4}(0.884 \mathrm{~mL}$ of a $2.5 \mathrm{wt} \%$ solution in $t-\mathrm{BuOH}, 0.0705 \mathrm{mmol})$, and the reaction mixture was stirred at room temperature for $18 \mathrm{~h}$. The reaction mixture was extracted with EtOAc. The extract was washed with water and brine, dried, and concentrated under reduced pressure. The residue was purified by flash chromatography (30\% EtOAc in hexane) to give hydroxy ketone $\mathbf{2 3}$ (382 $\mathrm{mg}, 91 \%$ ) as a colorless oil. $[\alpha]^{26}+23.3\left(c\right.$ 1.12, $\left.\mathrm{CHCl}_{3}\right)$; IR (film) 3482, 2942, 2867, 1714, 1454, $1098 \mathrm{~cm}^{-1} ;{ }^{1} \mathrm{H}$ NMR (600 MHz, $\left.\mathrm{CDCl}_{3}\right) \delta 7.36-7.26(10 \mathrm{H}, \mathrm{m}), 4.67$ and $4.50($ each $1 \mathrm{H}, \mathrm{d}, J=11.7 \mathrm{~Hz}), 4.51(1 \mathrm{H}, \mathrm{ddd}, J=12.5,4.6,3.7$ $\mathrm{Hz}), 4.50$ and 4.49 (each 1H, d, $J=11.9 \mathrm{~Hz}), 4.15(1 \mathrm{H}, \mathrm{dd}, J=6.6,5.4 \mathrm{~Hz}), 4.02(1 \mathrm{H}, \mathrm{td}, J=5.4,3.2 \mathrm{~Hz})$, $3.88(1 \mathrm{H}, \mathrm{ddt}, J=11.7,4.6,1.3 \mathrm{~Hz}), 3.84(1 \mathrm{H}, \mathrm{ddd}, J=8.8,4.0,3.2 \mathrm{~Hz}), 3.50(1 \mathrm{H}, \mathrm{dd}, J=9.9,5.4 \mathrm{~Hz})$, $3.48(1 \mathrm{H}, \mathrm{dd}, J=9.9,5.4 \mathrm{~Hz}), 3.40(1 \mathrm{H}, \mathrm{d}, J=4.6 \mathrm{~Hz}), 3.34(1 \mathrm{H}, \mathrm{td}, J=11.7,2.6 \mathrm{~Hz}), 3.29(1 \mathrm{H}, \mathrm{ddd}, J$ $=11.2,9.1,4.2 \mathrm{~Hz}), 2.89(1 \mathrm{H}, \mathrm{ddd}, J=10.8,9.1,4.6 \mathrm{~Hz}), 2.27(1 \mathrm{H}, \mathrm{ddd}, J=13.4,4.2,3.7 \mathrm{~Hz}), 2.17(1 \mathrm{H}$, ddd, $J=14.5,8.8,5.4 \mathrm{~Hz}), 2.08(1 \mathrm{H}, \mathrm{ddtd}, J=12.7,4.6,4.3,1.3 \mathrm{~Hz}), 1.99(1 \mathrm{H}, \mathrm{ddd}, J=14.5,6.6,4.0$ Hz), 1.69 (1H, ddddd, $J=13.7,4.8,4.3,2.6,1.3 \mathrm{~Hz}), 1.62(1 \mathrm{H}$, dddt, $J=13.7,12.7,11.7,4.3 \mathrm{~Hz}), 1.55$ $(1 \mathrm{H}, \mathrm{ddd}, J=13.4,12.5,11.2 \mathrm{~Hz}), 1.48(1 \mathrm{H}, \mathrm{tdd}, J=12.7,10.8,4.8 \mathrm{~Hz}), 1.00-0.91(7 \mathrm{H}, \mathrm{m}), 0.95(3 \mathrm{H}, \mathrm{t}$, $J=8.1 \mathrm{~Hz}), 0.94(3 \mathrm{H}, \mathrm{t}, J=8.1 \mathrm{~Hz}), 0.65-0.51(4 \mathrm{H}, \mathrm{m}) ;{ }^{13} \mathrm{C} \mathrm{NMR}\left(150 \mathrm{MHz}, \mathrm{CDCl}_{3}\right) \delta 215.0,138.4$ 
$138.2,128.3,128.2,127.7,127.6,127.5,127.4,83.2,82.1,78.8,77.0,73.4,73.2,72.0,71.8,71.0,67.6$, 39.3, 34.9, 30.9, 25.5, $17.3(\times 2), 13.0,7.1(\times 2), 3.8,3.7$; HRFABMS $m / z$ calcd for $\mathrm{C}_{34} \mathrm{H}_{50} \mathrm{O}_{7} \mathrm{SiNa}\left(\mathrm{MNa}^{+}\right)$ 621.3224, found 621.3245 .

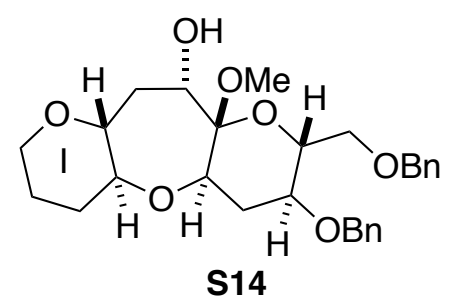

Hydroxy acetal S14. (i) Methyl acetalization: To a solution of hydroxy ketone 23 (380 mg, 0.635 mmol) in $\mathrm{CH}_{2} \mathrm{Cl}_{2}-\mathrm{MeOH}-\mathrm{CH}(\mathrm{OMe})_{3}(10: 2: 10,12.7 \mathrm{~mL})$ was added $p-\mathrm{TsOH} \cdot \mathrm{H}_{2} \mathrm{O}(242 \mathrm{mg}, 1.27 \mathrm{mmol})$, and the reaction mixture was stirred for $1 \mathrm{~h}$. The reaction was quenched with $\mathrm{Et}_{3} \mathrm{~N}(2 \mathrm{~mL})$, and the mixture was concentrated under the reduced pressure to give a crude methyl acetal containing a small amount of dimethyl orthoester of the hydroxy group.

(ii) Methanolysis of the Orthoester: $\mathrm{Et}_{3} \mathrm{~N}$ in the crude acetal was completely removed by azeotropic evaporation with EtOAc. This crude acetal was dissolved in $\mathrm{CH}_{2} \mathrm{Cl}_{2} / \mathrm{MeOH}(1: 1,12.7 \mathrm{~mL})$ and PPTS (79.8 $\mathrm{mg}, 0.317 \mathrm{mmol}$ ) was added. The reaction mixture was stirred at room temperature for $40 \mathrm{~min}$. The reaction was quenched with $\mathrm{Et}_{3} \mathrm{~N}(2 \mathrm{~mL})$, and the reaction mixture was concentrated under reduced pressure Purification by flash chromatography (60\% EtOAc in hexane) afforded hydroxy methyl acetal S14 (245 mg, 80\%) as a colorless solid. Mp 102-103 ${ }^{\circ} \mathrm{C} ;[\alpha]^{26} \mathrm{D}+86.8\left(c 1.24, \mathrm{CHCl}_{3}\right)$; IR (film) 3448, 2940, 2861, 1086, $1036 \mathrm{~cm}^{-1} ;{ }^{1} \mathrm{H}$ NMR (600 MHz, $\left.\mathrm{CDCl}_{3}\right) \delta$ 7.35-7.24 (10H, m), 4.63 and 4.41 (each 1H, $\mathrm{d}, J=11.4 \mathrm{~Hz}), 4.57$ and $4.52($ each $1 \mathrm{H}, \mathrm{d}, J=12.3 \mathrm{~Hz}), 4.11(1 \mathrm{H}, \mathrm{dt}, J=7.2,4.1 \mathrm{~Hz}), 3.87(1 \mathrm{H}, \mathrm{ddt}, J=$ 10.9, 3.5, $2.2 \mathrm{~Hz}), 3.75(1 \mathrm{H}, \mathrm{dd}, J=12.5,4.4 \mathrm{~Hz}), 3.73(1 \mathrm{H}, \mathrm{dd}, J=10.6,1.8 \mathrm{~Hz}), 3.69(1 \mathrm{H}, \mathrm{dd}, J=10.6$, $4.8 \mathrm{~Hz}), 3.62(1 \mathrm{H}, \mathrm{ddd}, J=9.7,4.8,1.8 \mathrm{~Hz}), 3.52(1 \mathrm{H}, \mathrm{ddd}, J=11.0,9.7,4.9 \mathrm{~Hz}), 3.41(1 \mathrm{H}, \mathrm{ddd}, J=10.9$, 9.3, 4.4 Hz), $3.32(3 \mathrm{H}, \mathrm{s}), 3.30-3.24(2 \mathrm{H}, \mathrm{m}), 2.74(1 \mathrm{H}, \mathrm{d}, J=4.0 \mathrm{~Hz}), 2.33(1 \mathrm{H}, \mathrm{ddd}, J=15.0,5.9,3.9$ $\mathrm{Hz}), 2.31(1 \mathrm{H}, \mathrm{dt}, J=11.0,4.6 \mathrm{~Hz}), 2.05(1 \mathrm{H}, \mathrm{m}), 1.98(1 \mathrm{H}, \mathrm{dt}, J=12.5,11.0 \mathrm{~Hz}), 1.92(1 \mathrm{H}, \mathrm{dt}, J=15.0$, $7.2 \mathrm{~Hz}), 1.72-1.62(2 \mathrm{H}, \mathrm{m}), 1.47(1 \mathrm{H}, \mathrm{tdd}, J=12.3,11.2,5.9 \mathrm{~Hz}) ;{ }^{13} \mathrm{C} \mathrm{NMR}\left(150 \mathrm{MHz}, \mathrm{CDCl}_{3}\right) \delta 138.2$, 
$137.9,128.43,128.40,127.8(\times 2), 127.7,127.6,98.8,80.3,79.8,75.7,73.3,72.9,72.1,70.8,70.6,69.0$, 67.9, 48.3, 37.0, 31.5, 31.2, 25.7; HRFABMS $m / z$ calcd for $\mathrm{C}_{28} \mathrm{H}_{36} \mathrm{O}_{7} \mathrm{Na}\left(\mathrm{MNa}^{+}\right)$507.2359, found 507.2377 .

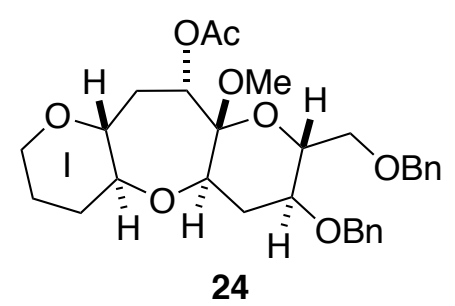

Acetoxy acetal 24. To a solution of hydroxy acetal $\mathbf{S 1 4}(237 \mathrm{mg}, 0.489 \mathrm{mmol})$ in pyridine $(1.3 \mathrm{~mL}$, $12.4 \mathrm{mmol})$ and acetic anhydride $(1.0 \mathrm{~mL}, 10.6 \mathrm{mmol})$ was added DMAP $(6.0 \mathrm{mg}, 0.049 \mathrm{mmol})$. The mixture was stirred for $2 \mathrm{~h}$, and then concentrated under reduced pressure. Flash chromatography $(40 \%$ EtOAc in $n$-hexane) afforded acetoxy acetal $24(247 \mathrm{mg}, 96 \%)$ as a colorless solid. $\mathrm{Mp} 117-119{ }^{\circ} \mathrm{C}$; $[\alpha]^{26} \mathrm{D}+99.6\left(c\right.$ 0.89, $\left.\mathrm{CHCl}_{3}\right)$; IR (film) 2941, 2861, 1741, 1238, 1086, $1040 \mathrm{~cm}^{-1} ;{ }^{1} \mathrm{H} \mathrm{NMR}(600 \mathrm{MHz}$, $\left.\mathrm{CDCl}_{3}\right) \delta 7.34-7.25(10 \mathrm{H}, \mathrm{m}), 5.38(1 \mathrm{H}, \mathrm{t}, J=4.1 \mathrm{~Hz}), 4.64$ and $4.47($ each $1 \mathrm{H}, \mathrm{d}, J=11.6 \mathrm{~Hz}), 4.59$ and $4.53($ each $1 \mathrm{H}, \mathrm{d}, J=12.3 \mathrm{~Hz}), 3.83(1 \mathrm{H}, \mathrm{dd}, J=12.3,4.4 \mathrm{~Hz}), 3.82(1 \mathrm{H}, \mathrm{ddt}, J=10.4,3.5,1.8 \mathrm{~Hz}), 3.72$ $(1 \mathrm{H}, \mathrm{dd}, J=11.2,4.0 \mathrm{~Hz}), 3.69(1 \mathrm{H}, \mathrm{dd}, J=11.2,1.7 \mathrm{~Hz}), 3.56(1 \mathrm{H}, \mathrm{td}, J=10.0,4.4 \mathrm{~Hz}), 3.53(1 \mathrm{H}, \mathrm{ddd}$, $J=10.0,4.0,1.7 \mathrm{~Hz}), 3.52(1 \mathrm{H}, \mathrm{ddd}, J=10.8,9.0,4.6 \mathrm{~Hz}), 3.44(1 \mathrm{H}, \mathrm{td}, J=9.1,4.2 \mathrm{~Hz}), 3.34(3 \mathrm{H}, \mathrm{s})$, $3.25(1 \mathrm{H}, \mathrm{m}), 2.51(1 \mathrm{H}, \mathrm{ddd}, J=16.3,8.5,3.9 \mathrm{~Hz}), 2.32(1 \mathrm{H}, \mathrm{dt}, J=11.3,4.2 \mathrm{~Hz}), 2.08(1 \mathrm{H}, \mathrm{m}), 2.01$ $(3 \mathrm{H}, \mathrm{s}), 1.93(1 \mathrm{H}, \mathrm{td}, J=11.6,10.9 \mathrm{~Hz}), 1.84(1 \mathrm{H}, \mathrm{dt}, J=16.4,4.3 \mathrm{~Hz}), 1.65-1.60(2 \mathrm{H}, \mathrm{m}), 1.48(1 \mathrm{H}, \mathrm{tdd}$, $J=12.3,10.8,5.9 \mathrm{~Hz}) ;{ }^{13} \mathrm{C} \mathrm{NMR}\left(150 \mathrm{MHz}, \mathrm{CDCl}_{3}\right) \delta 169.5,138.8,138.1,128.4,128.3,127.73,127.69$, $127.3,127.2,98.5,79.5,78.2,75.4,73.6,73.2,71.9,71.2,70.9,68.6,67.7,48.4,33.8,31.6,31.4,25.7$, 21.3; HRFABMS $m / z$ calcd for $\mathrm{C}_{30} \mathrm{H}_{38} \mathrm{O}_{8} \mathrm{Na}\left(\mathrm{MNa}^{+}\right)$549.2464, found 549.2484. 


\section{Model compound 31 for the IJK ring system (Scheme 5(b))}

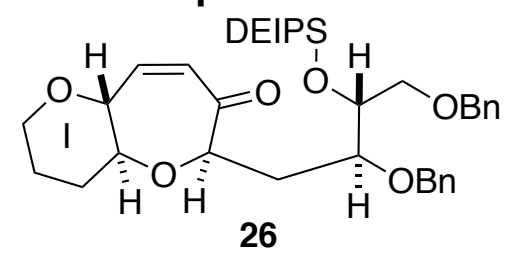

Conjugated ketone 26. To a solution of the 7-membered ketone $22(502 \mathrm{mg}, 0.863 \mathrm{mmol})$ in THF (22 $\mathrm{mL})$ and $\mathrm{Et}_{3} \mathrm{~N}(0.602 \mathrm{~mL}, 4.32 \mathrm{mmol})$ at $-80^{\circ} \mathrm{C}$ was added $\mathrm{TESCl}(0.725 \mathrm{~mL}, 4.32 \mathrm{mmol})$ and LiHMDS (2.6 mL of $1.0 \mathrm{M}$ solution in THF, $2.6 \mathrm{mmol}$ ). The reaction mixture was stirred at $-80{ }^{\circ} \mathrm{C}$ for $1.5 \mathrm{~h}$. The reaction was quenched with phosphate buffer $(\mathrm{pH} 7)$ and the reaction mixture was extracted with EtOAc. The extract was washed with brine, dried, and concentrated under reduced pressure to afford a crude silyl enol ether $(780 \mathrm{mg})$. To a solution of the silyl enol ether in $\mathrm{CH}_{3} \mathrm{CN}(86 \mathrm{~mL})$ was added $\mathrm{Pd}(\mathrm{OAc})_{2}(500$ $\mathrm{mg}, 2.18 \mathrm{mmol}$ ), and the reaction mixture was stirred at room temperature for $2.5 \mathrm{~h}$. The mixture was filtered through a Celite pad, and the filtrate was concentrated under reduced pressure. Flash chromatography ( $2 \%$ acetone in benzene) afforded the conjugated ketone $\mathbf{2 6}(400 \mathrm{mg}, 80 \%)$ as a colorless oil. $[\alpha]^{28} \mathrm{D}-50.6\left(c 1.18, \mathrm{CHCl}_{3}\right)$; IR (film) 2942, 2872, 1667, 1454, 1128, $1092 \mathrm{~cm}^{-1}$; ${ }^{1} \mathrm{H} \mathrm{NMR}(600 \mathrm{MHz}$, $\left.\mathrm{CDCl}_{3}\right) \delta 7.36-7.22(10 \mathrm{H}, \mathrm{m}), 6.21(1 \mathrm{H}, \mathrm{dd}, J=12.8,2.4 \mathrm{~Hz}), 5.78(1 \mathrm{H}, \mathrm{dd}, J=12.8,2.6 \mathrm{~Hz}), 4.68$ and $4.46($ each $1 \mathrm{H}, \mathrm{d}, J=11.6 \mathrm{~Hz}), 4.51$ and $4.49($ each $1 \mathrm{H}, \mathrm{d}, J=12.1 \mathrm{~Hz}), 4.28(1 \mathrm{H}, \mathrm{dd}, J=6.2,4.8 \mathrm{~Hz})$, $4.00(1 \mathrm{H}, \mathrm{ddd}, J=5.7,5.1,3.0 \mathrm{~Hz}), 3.93(1 \mathrm{H}, \mathrm{ddt}, J=11.3,4.4,1.4 \mathrm{~Hz}), 3.85(1 \mathrm{H}, \mathrm{ddd}, J=8.7,3.9,3.0$ Hz), $3.78(1 \mathrm{H}, \mathrm{ddd}, J=8.8,2.6,2.4 \mathrm{~Hz}), 3.53(1 \mathrm{H}, \mathrm{dd}, J=9.9,5.1 \mathrm{~Hz}), 3.48(1 \mathrm{H}, \mathrm{dd}, J=9.9,5.7 \mathrm{~Hz})$, $3.40(1 \mathrm{H}, \mathrm{td}, J=11.5,3.1 \mathrm{~Hz}), 3.31(1 \mathrm{H}, \mathrm{ddd}, J=10.9,8.8,4.8 \mathrm{~Hz}), 2.17(1 \mathrm{H}, \mathrm{ddd}, J=14.6,8.8,4.8 \mathrm{~Hz})$, $2.15(1 \mathrm{H}, \mathrm{m}), 2.03(1 \mathrm{H}, \mathrm{ddd}, J=14.6,6.2,3.9 \mathrm{~Hz}), 1.74-1.62(2 \mathrm{H}, \mathrm{m}), 1.55(1 \mathrm{H}, \mathrm{tdd}, J=12.5,10.9,5.3$ $\mathrm{Hz}), 0.99-0.91(13 \mathrm{H}, \mathrm{m}), 0.66-0.56(4 \mathrm{H}, \mathrm{m}) ;{ }^{13} \mathrm{C} \mathrm{NMR}\left(150 \mathrm{MHz}, \mathrm{CDCl}_{3}\right) \delta 203.7,142.8,138.8,138.3$, $128.3,128.2,128.1,127.6,127.5,127.2,127.1,84.2,80.5,78.3,77.4,73.5,73.3,72.0,71.9,68.1,35.2$, 30.6, 25.4, $17.3(\times 2), 12.9,7.07,7.06,3.8,3.7 ;$ HRFABMS $m / z$ calcd for $\mathrm{C}_{34} \mathrm{H}_{48} \mathrm{O}_{6} \mathrm{SiNa}\left(\mathrm{MNa}^{+}\right) 603.3118$, found 603.3141 . 


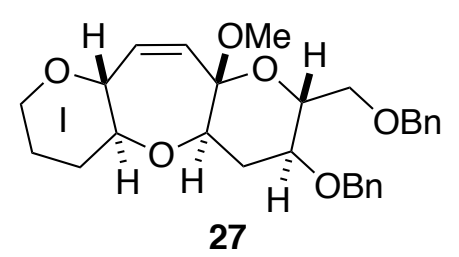

Methyl acetal 27. To a solution of the conjugated ketone 26 (398 mg, $0.686 \mathrm{mmol})$ in $\mathrm{CH}_{2} \mathrm{Cl}_{2}-\mathrm{MeOH}-$ $\mathrm{CH}(\mathrm{OMe})_{3}(10: 2: 10,13.7 \mathrm{~mL})$ was added $p-\mathrm{TsOH} \cdot \mathrm{H}_{2} \mathrm{O}(261 \mathrm{mg}, 1.37 \mathrm{mmol})$, and the reaction mixture was stirred at room temperature for $0.8 \mathrm{~h}$. The reaction was quenched with $\mathrm{Et}_{3} \mathrm{~N}$, and the mixture was concentrated under the reduced pressure. Flash chromatography (30\% EtOAc in $n$-hexane) afforded methyl acetal $27(297 \mathrm{mg}, 93 \%)$ as a colorless solid. Mp $73-75{ }^{\circ} \mathrm{C} ;[\alpha]^{26}{ }_{\mathrm{D}}+59.0\left(\mathrm{c} 2.02, \mathrm{CHCl}_{3}\right)$; $\mathrm{IR}$ (film) 2939, 2863, 1453, 1100, $1075 \mathrm{~cm}^{-1} ;{ }^{1} \mathrm{H}$ NMR (600 MHz, $\left.\mathrm{CDCl}_{3}\right) \delta 7.35-7.21(10 \mathrm{H}, \mathrm{m}), 5.75(1 \mathrm{H}$, $\mathrm{dd}, J=12.7,2.0 \mathrm{~Hz}), 5.68(1 \mathrm{H}, \mathrm{dd}, J=12.7,2.8 \mathrm{~Hz}), 4.63$ and $4.56($ each $1 \mathrm{H}, \mathrm{d}, J=12.3 \mathrm{~Hz}), 4.59$ and $4.40($ each $1 \mathrm{H}, \mathrm{d}, J=11.6 \mathrm{~Hz}), 3.99(1 \mathrm{H}, \mathrm{ddd}, J=9.2,2.8,2.0 \mathrm{~Hz}), 3.87(1 \mathrm{H}, \mathrm{ddt}, J=11.7,3.3,2.0 \mathrm{~Hz})$, $3.72(1 \mathrm{H}, \mathrm{dd}, J=10.8,2.4 \mathrm{~Hz}), 3.70(1 \mathrm{H}, \mathrm{dd}, J=10.8,4.2 \mathrm{~Hz}), 3.63(1 \mathrm{H}, \mathrm{ddd}, J=9.7,4.2,2.4 \mathrm{~Hz}), 3.56$ $(1 \mathrm{H}, \mathrm{dd}, J=12.1,4.8 \mathrm{~Hz}), 3.53(1 \mathrm{H}, \mathrm{ddd}, J=11.2,9.7,4.8 \mathrm{~Hz}), 3.32-3.26(2 \mathrm{H}, \mathrm{m}), 3.26(3 \mathrm{H}, \mathrm{s}), 2.26$ $(1 \mathrm{H}, \mathrm{dt}, J=11.6,4.6 \mathrm{~Hz}), 2.04(1 \mathrm{H}, \mathrm{m}), 1.87(1 \mathrm{H}, \mathrm{q}, J=11.7 \mathrm{~Hz}), 1.67-1.54(3 \mathrm{H}, \mathrm{m}) ;{ }^{13} \mathrm{C}$ NMR $(150$ $\left.\mathrm{MHz}, \mathrm{CDCl}_{3}\right) \delta 138.4,138.1,136.7,131.0,128.33,128.29,127.72,127.65,127.6,127.5,98.3,79.9$, $78.79,78.77,73.4,71.9,71.8,70.7,68.9,67.2,48.9,32.5,30.8,25.2$; HRFABMS $m / z$ calcd for $\mathrm{C}_{28} \mathrm{H}_{34} \mathrm{O}_{6} \mathrm{Na}\left(\mathrm{MNa}^{+}\right) 489.2253$, found 489.2249.

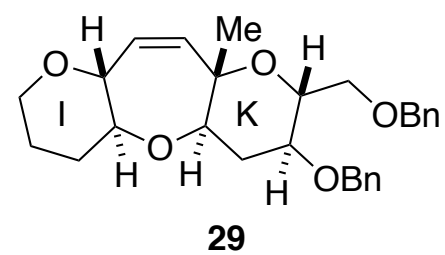

Methylated product 29. To a solution of methyl acetal $27(103 \mathrm{mg}, 0.221 \mathrm{mmol})$ in $\mathrm{CH}_{2} \mathrm{Cl}_{2}(2.2 \mathrm{~mL})$ at $-20{ }^{\circ} \mathrm{C}$ were added $\mathrm{AlMe}_{3}(1.03 \mathrm{~mL}$ of $1.07 \mathrm{M}$ solution in $n$-hexane, $1.10 \mathrm{mmol})$ and $\mathrm{BF}_{3} \cdot \mathrm{OEt}_{2}(0.136$ $\mathrm{mL}, 1.10 \mathrm{mmol})$. The reaction mixture was stirred at $-20^{\circ} \mathrm{C}$ for $0.5 \mathrm{~h}$, and the reaction was quenched with saturated aqueous $\mathrm{NaHCO}_{3}$ solution. After addition of $10 \%$ potassium sodium tartrate solution, the 
mixture was extracted with EtOAc. The extract was washed with brine, dried, and concentrated under reduced pressure. Flash chromatography (10\% EtOAc in $n$-hexane) afforded the methylated product 29 (69 mg, 69\%) as a colorless solid. Mp $72-74{ }^{\circ} \mathrm{C} ;[\alpha]^{26} \mathrm{D}+62.2\left(c \mathrm{c} 0.68, \mathrm{CHCl}_{3}\right)$; IR (film) 2940, 2864, $1454,1092 \mathrm{~cm}^{-1} ;{ }^{1} \mathrm{H}$ NMR (600 MHz, $\left.\mathrm{CDCl}_{3}\right) \delta 7.35-7.20(10 \mathrm{H}, \mathrm{m}), 5.82(1 \mathrm{H}, \mathrm{dd}, J=12.8,2.8 \mathrm{~Hz}), 5.43$ $(1 \mathrm{H}, \mathrm{dd}, J=12.7,1.8 \mathrm{~Hz}), 4.62$ and $4.57($ each $1 \mathrm{H}, \mathrm{d}, J=12.5 \mathrm{~Hz}$ ), 4.58 and 4.38 (each 1H, d, $J=11.4$ Hz), $3.89(1 \mathrm{H}, \mathrm{ddt}, J=11.2,3.7,1.7 \mathrm{~Hz}), 3.81(1 \mathrm{H}, \mathrm{ddd}, J=9.1,2.8,1.8 \mathrm{~Hz}), 3.69(1 \mathrm{H}, \mathrm{dd}, J=10.8,2.0$ Hz), $3.63(1 \mathrm{H}, \mathrm{dd}, J=10.8,4.8 \mathrm{~Hz}), 3.56(1 \mathrm{H}, \mathrm{ddd}, J=9.7,4.8,2.0 \mathrm{~Hz}), 3.50(1 \mathrm{H}, \mathrm{dd}, J=12.3,4.4 \mathrm{~Hz})$, $3.45(1 \mathrm{H}, \mathrm{ddd}, J=11.2,9.7,5.0 \mathrm{~Hz}), 3.30(1 \mathrm{H}, \mathrm{m}), 3.26(1 \mathrm{H}, \mathrm{ddd}, J=11.0,9.2,4.6 \mathrm{~Hz}), 2.32(1 \mathrm{H}, \mathrm{ddd}$, $J=12.0,5.0,4.3 \mathrm{~Hz}), 2.04(1 \mathrm{H}, \mathrm{m}), 1.71-1.62(2 \mathrm{H}, \mathrm{m}), 1.62(1 \mathrm{H}, \mathrm{td}, J=12.1,11.4 \mathrm{~Hz}), 1.48(1 \mathrm{H}, \mathrm{m})$, $1.33(3 \mathrm{H}, \mathrm{s}) ;{ }^{13} \mathrm{C} \mathrm{NMR}\left(150 \mathrm{MHz}, \mathrm{CDCl}_{3}\right) \delta 138.9,138.5,138.0,129.7,128.4,128.3,127.8(\times 2), 127.7$, $127.5,80.5,80.1,79.7,78.1,73.4,72.8,72.5,70.9,69.6,67.5,33.0,31.2,25.5,15.0 ;$ HRFABMS $m / z$ calcd for $\mathrm{C}_{28} \mathrm{H}_{34} \mathrm{O}_{5} \mathrm{Na}\left(\mathrm{MNa}^{+}\right)$473.2304, found 473.2315.

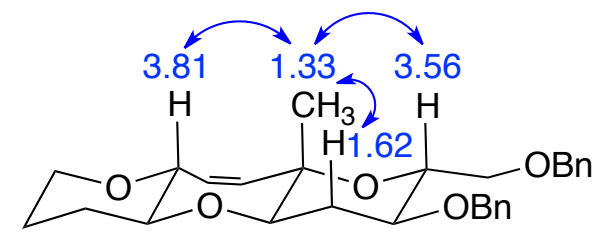

\section{Figure S1. NOESY correlation of 29}

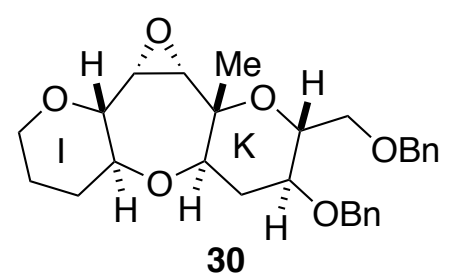

Epoxide 30. To a solution of alkene 29 (74.4 mg, $0.165 \mathrm{mmol})$ in $\mathrm{CH}_{2} \mathrm{Cl}_{2}(1 \mathrm{~mL})$ was added $m$-CPBA (57 mg, $0.33 \mathrm{mmol}$ ), and the reaction mixture was stirred at room temperature for $46 \mathrm{~h}$. The reaction was quenched with saturated aqueous $\mathrm{Na}_{2} \mathrm{~S}_{2} \mathrm{O}_{3}$ solution, and the mixture was extracted with EtOAc. The extract was washed with saturated aqueous $\mathrm{NaHCO}_{3}$ solution and brine, dried, and concentrated under 
reduced pressure. Flash chromatography (30\% EtOAc in $n$-hexane) afforded major $\alpha$-epoxide 30 (61.4 $\mathrm{mg}, 80 \%$ ) and minor $\beta$-epoxide $\mathbf{3 0}^{\prime}$ (6.0 $\mathrm{mg}, 8 \%$ ).

$\alpha$-Epoxide 30: Colorless oil; $[\alpha]^{26}{ }_{\mathrm{D}}+62.8\left(\mathrm{c} 1.12, \mathrm{CHCl}_{3}\right)$; IR (film) 2944, 2854, 1454, $1087 \mathrm{~cm}^{-1} ;{ }^{1} \mathrm{H}$ $\operatorname{NMR}\left(600 \mathrm{MHz}, \mathrm{CDCl}_{3}\right) \delta 7.36-7.26(8 \mathrm{H}, \mathrm{m}), 7.24-7.20(2 \mathrm{H}, \mathrm{m}), 4.61$ and 4.58 (each $1 \mathrm{H}, \mathrm{d}, J=12.3$ Hz), 4.58 and 4.39 (each 1H, d, $J=11.4 \mathrm{~Hz}), 3.97(1 \mathrm{H}, \mathrm{ddt}, J=11.1,4.4,1.6 \mathrm{~Hz}), 3.73(1 \mathrm{H}, \mathrm{m}), 3.64-$ $3.59(2 \mathrm{H}, \mathrm{m}), 3.54(1 \mathrm{H}, \mathrm{dd}, J=12.5,4.6 \mathrm{~Hz}), 3.383(1 \mathrm{H}, \mathrm{d}, J=9.2 \mathrm{~Hz}), 3.380(1 \mathrm{H}, \mathrm{ddd}, J=11.4,9.1,4.7$ Hz), $3.35(1 \mathrm{H}, \mathrm{ddd}, J=11.0,9.1,4.6 \mathrm{~Hz}), 3.31(1 \mathrm{H}, \mathrm{td}, J=11.4,3.2 \mathrm{~Hz}), 3.23(2 \mathrm{H}, \mathrm{s}), 2.32(1 \mathrm{H}, \mathrm{dt}, J=$ 12.1, $4.7 \mathrm{~Hz}), 2.06(1 \mathrm{H}, \mathrm{m}), 1.74-1.64(2 \mathrm{H}, \mathrm{m}), 1.61(1 \mathrm{H}, \mathrm{td}, J=12.2,11.4 \mathrm{~Hz}), 1.39(1 \mathrm{H}, \mathrm{tdd}, J=12.6$, 12.6, 10.8, $5.2 \mathrm{~Hz}), 1.27(3 \mathrm{H}, \mathrm{s}) ;{ }^{13} \mathrm{C} \mathrm{NMR}\left(150 \mathrm{MHz}, \mathrm{CDCl}_{3}\right) \delta 138.6,138.0,128.4,128.3,127.8,127.7$ $(\times 2), 127.4,79.8,76.6,75.2,74.2,73.4,73.2,72.9,71.0,69.9,68.3,60.5,56.8,32.1,30.9,25.5,13.9$; HRFABMS $m / z$ calcd for $\mathrm{C}_{28} \mathrm{H}_{34} \mathrm{O}_{6} \mathrm{Na}\left(\mathrm{MNa}^{+}\right)$489.2253, found 489.2257 .

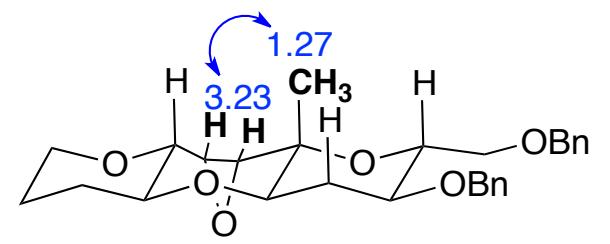

\section{Figure S2. NOESY correlation of 30}

Minor $\beta$-epoxide 30': Colorless oil; $[\alpha]^{25} \mathrm{D}+44.6$ (c 0.13, $\mathrm{CHCl}_{3}$ ); IR (film) 2848, 1416, $1087 \mathrm{~cm}^{-1} ;{ }^{1} \mathrm{H}$ NMR (600 MHz, $\left.\mathrm{CDCl}_{3}\right) \delta 7.35-7.26(8 \mathrm{H}, \mathrm{m}), 7.24-7.22(2 \mathrm{H}, \mathrm{m}), 4.63$ and 4.57 (each $1 \mathrm{H}, \mathrm{d}, J=12.3$ Hz), 4.58 and 4.41 (each 1H, d, $J=11.4 \mathrm{~Hz}), 3.90(1 \mathrm{H}, \mathrm{m}), 3.69(2 \mathrm{H}, \mathrm{d}, J=3.1 \mathrm{~Hz}), 3.57(1 \mathrm{H}, \mathrm{dt}, J=9.5$, $3.1 \mathrm{~Hz}), 3.51(1 \mathrm{H}, \mathrm{ddd}, J=11.4,9.9,4.8 \mathrm{~Hz}), 3.43(1 \mathrm{H}, \mathrm{dd}, J=12.1,4.8 \mathrm{~Hz}), 3.29(1 \mathrm{H}, \mathrm{m}), 3.24(1 \mathrm{H}, \mathrm{td}$, $J=9.7,5.1 \mathrm{~Hz}), 3.22(1 \mathrm{H}, \mathrm{d}, J=5.2 \mathrm{~Hz}), 3.21(1 \mathrm{H}, \mathrm{dd}, J=9.7,4.1 \mathrm{~Hz}), 3.00(1 \mathrm{H}, \mathrm{dd}, J=5.2,4.1 \mathrm{~Hz})$, $2.33(1 \mathrm{H}, \mathrm{dt}, J=12.3,4.8 \mathrm{~Hz}), 2.08(1 \mathrm{H}, \mathrm{m}), 1.66-1.61(2 \mathrm{H}, \mathrm{m}), 1.61(1 \mathrm{H}, \mathrm{td}, J=12.2,11.4 \mathrm{~Hz}), 1.39$ $(1 \mathrm{H}$, dddd, $J=12.8,11.0,9.9,7.0 \mathrm{~Hz}), 1.14(3 \mathrm{H}, \mathrm{s}) ;{ }^{13} \mathrm{C} \mathrm{NMR}\left(150 \mathrm{MHz}, \mathrm{CDCl}_{3}\right) \delta 138.4,138.0,128.4$, $128.3,127.8,127.73,127.72,127.5,80.6,78.1,76.5,76.4,73.5,72.8,72.1,71.0,69.3,67.0,61.8,56.9$, 33.0, 30.8, 24.9, 12.6; HRFABMS $m / z$ calcd for $\mathrm{C}_{28} \mathrm{H}_{34} \mathrm{O}_{6} \mathrm{Na}\left(\mathrm{MNa}^{+}\right)$489.2253, found 489.2266. 


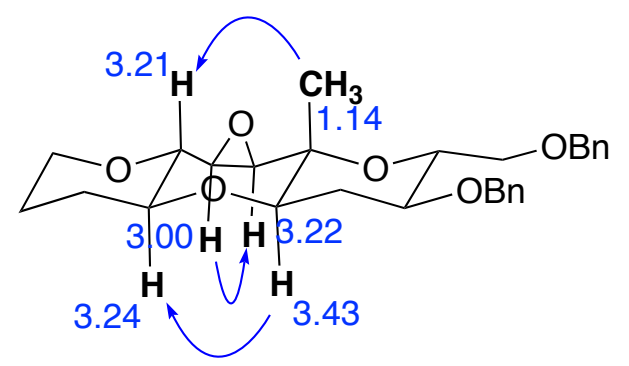

An NOE was not observed between 1.14 and $3.22 \mathrm{ppm}$.

\section{Figure S3. NOESY correlation of 30'}

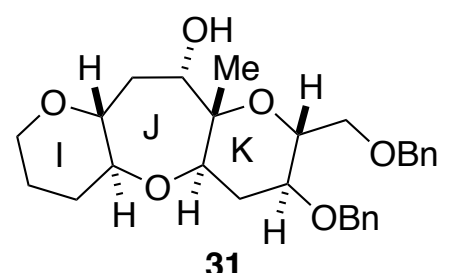

Alcohol 31. To a solution of epoxide $30(9.9 \mathrm{mg}, 0.021 \mathrm{mmol})$ in THF $(1 \mathrm{~mL})$ was added $\mathrm{LiBEt}_{3} \mathrm{H}$ ( $0.606 \mathrm{~mL}$ of $1.05 \mathrm{M}$ solution in THF, $0.637 \mathrm{mmol}$ ), and the mixture was stirred at room temperature for $4 \mathrm{~h}$. The mixture was quenched with $10 \%$ aqueous potassium sodium tartrate, and the mixture was extracted with EtOAc. The extract was washed with brine, dried, and concentrated under the reduced pressure. Flash chromatography (40\% EtOAc in $n$-hexane) afforded alcohol 31 (10.1 mg, quant.) as a colorless oil. $[\alpha]^{26}{ }_{\mathrm{D}}+50.5\left(\mathrm{c} 0.84, \mathrm{CHCl}_{3}\right)$; IR (film) 3450, 2941, 2859, $1087 \mathrm{~cm}^{-1} ;{ }^{1} \mathrm{H} \mathrm{NMR}(600 \mathrm{MHz}$, $\left.\mathrm{CDCl}_{3}\right) \delta 7.37-7.29(8 \mathrm{H}, \mathrm{m}), 7.27-7.25(2 \mathrm{H}, \mathrm{m}), 4.63$ and $4.42($ each $1 \mathrm{H}, \mathrm{d}, J=11.5 \mathrm{~Hz}), 4.57$ and 4.53 (each $1 \mathrm{H}, \mathrm{d}, J=12.1 \mathrm{~Hz}), 3.83-3.89(3 \mathrm{H}, \mathrm{m}), 3.69(1 \mathrm{H}, \mathrm{dd}, J=10.5,2.2 \mathrm{~Hz}), 3.66(1 \mathrm{H}, \mathrm{dd}, J=10.5,4.3$ Hz), $3.63(1 \mathrm{H}, \mathrm{ddd}, J=9.5,4.3,2.2 \mathrm{~Hz}), 3.52(1 \mathrm{H}, \mathrm{ddd}, J=11.0,9.5,4.8 \mathrm{~Hz}), 3.49$ (1H, ddd, $J=11.0$, 9.5, $4.8 \mathrm{~Hz}), 3.28(1 \mathrm{H}, \mathrm{ddd}, J=9.2,7.9,6.2 \mathrm{~Hz}), 3.26(1 \mathrm{H}, \mathrm{td}, J=11.6,2.9 \mathrm{~Hz}), 2.94(1 \mathrm{H}, \mathrm{br} \mathrm{s}, \mathrm{OH}), 2.46$ (1H, ddd, $J=15.9,7.8,5.0 \mathrm{~Hz}), 2.40(1 \mathrm{H}, \mathrm{dt}, J=11.8,4.7 \mathrm{~Hz}), 2.09(1 \mathrm{H}, \mathrm{m}), 1.90(1 \mathrm{H}, \mathrm{ddd}, J=15.9$, 6.1, $4.4 \mathrm{~Hz}), 1.73-1.61(3 \mathrm{H}, \mathrm{m}), 1.38(1 \mathrm{H}, \mathrm{tdd}, J=12.7,11.3,5.0 \mathrm{~Hz}), 1.20(3 \mathrm{H}, \mathrm{s}) ;{ }^{13} \mathrm{C}$ NMR $(150 \mathrm{MHz}$, $\left.\mathrm{CDCl}_{3}\right) \delta 138.2,138.0,128.41,128.36,127.77,127.76,127.63,127.61,79.6,78.71,78.67,75.2,73.6$, 73.3, 72.7, 72.6, 70.9, 69.6, 67.5, 36.3, 32.1, 31.6, 25.6, 14.0; HRFABMS $m / z$ calcd for $\mathrm{C}_{28} \mathrm{H}_{36} \mathrm{O}_{6} \mathrm{Na}$ $\left(\mathrm{MNa}^{+}\right)$491.2410, found 491.2407. 


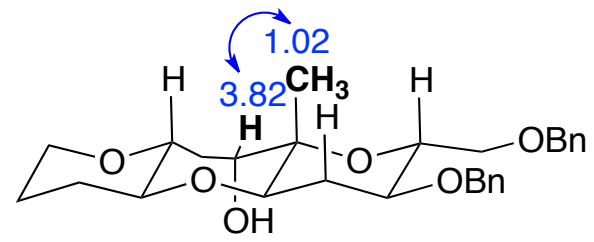

Figure S4. NOESY correlation of 31 in $\mathrm{C}_{6} \mathrm{D}_{6}$.

\section{Synthesis of the L-ring epoxy sulfone 3}

\section{Scheme S2. Synthesis of the L-ring epoxy sulfone 3.}
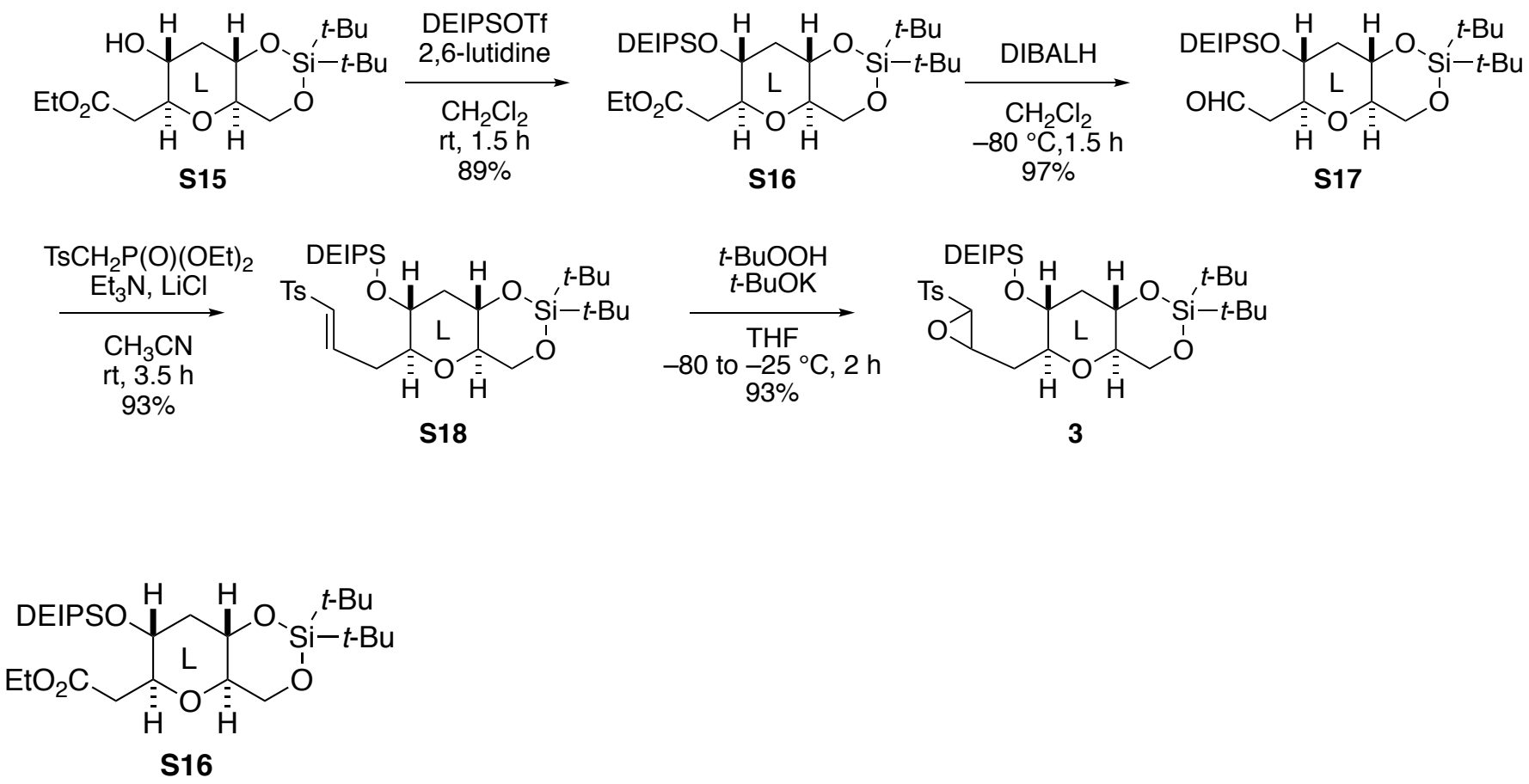

DEIPS ether S16. To a solution of hydroxy ester $\mathbf{S 1 5}^{5}$ (2.36 g, $6.29 \mathrm{mmol}, 1.0$ equiv) in $\mathrm{CH}_{2} \mathrm{Cl}_{2}(21 \mathrm{~mL})$ and 2,6-lutidine ( $1.10 \mathrm{~mL}, 9.49 \mathrm{mmol}, 1.5$ equiv) at $0{ }^{\circ} \mathrm{C}$ was added DEIPSOTf ( $1.67 \mathrm{~mL}, 1.1$ equiv), and the mixture was stirred at room temperature for $1.5 \mathrm{~h}$. The reaction was quenched with saturated aqueous $\mathrm{NaHCO}_{3}$ solution, and the resulting mixture was extracted with EtOAc. The organic layer was washed with brine, dried, and concentrated under reduced pressure. Flash chromatography on silica gel $(5 \%$ EtOAc in $n$-hexane) afforded DEIPS ether $\mathbf{S 1 6}(2.82 \mathrm{~g}, 89 \%)$ as a colorless oil. $[\alpha]^{24} \mathrm{D}-46.7(c 1.43$, $\left.\mathrm{CHCl}_{3}\right)$; IR (film) 2957, 2936, 2877, 2863, 1743, $1093 \mathrm{~cm}^{-1} ;{ }^{1} \mathrm{H} \mathrm{NMR}\left(600 \mathrm{MHz}, \mathrm{CDCl}_{3}\right) \delta 4.15$ (2H, q, $J=7.2 \mathrm{~Hz}), 4.08(1 \mathrm{H}, \mathrm{dd}, J=10.1,5.0 \mathrm{~Hz}), 3.75(1 \mathrm{H}, \mathrm{t}, J=10.1 \mathrm{~Hz}), 3.73(1 \mathrm{H}, \mathrm{ddd}, J=11.0,9.2,4.4$ 
Hz), $3.62(1 \mathrm{H}, \mathrm{td}, J=9.3,2.8 \mathrm{~Hz}), 3.47(1 \mathrm{H}, \mathrm{ddd}, J=10.8,9.1,4.5 \mathrm{~Hz}), 3.32(1 \mathrm{H}, \mathrm{ddd}, J=10.1,9.4,5.0$ Hz), $2.82(1 \mathrm{H}, \mathrm{dd}, J=15.2,2.8 \mathrm{~Hz}), 2.39(1 \mathrm{H}, \mathrm{dt}, J=12.0,4.5 \mathrm{~Hz}), 2.28(1 \mathrm{H}, \mathrm{dd}, J=15.4,9.5 \mathrm{~Hz}), 1.55$ $(1 \mathrm{H}, \mathrm{q}, J=11.3 \mathrm{~Hz}), 1.25(3 \mathrm{H}, \mathrm{t}, J=7.2 \mathrm{~Hz}), 1.03(9 \mathrm{H}, \mathrm{s}), 1.01-0.90(13 \mathrm{H}, \mathrm{m}), 0.98(9 \mathrm{H}, \mathrm{s}), 0.68-0.61$ $(4 \mathrm{H}, \mathrm{m}) ;{ }^{13} \mathrm{C} \mathrm{NMR}\left(150 \mathrm{MHz}, \mathrm{CDCl}_{3}\right) \delta 171.5,79.3,77.1,72.3,69.7,66.8,60.5,42.5,37.6,27.4,27.1$, 22.6, 19.9, 17.3 (x2), 14.2, 12.9, 7.10, 7.06, 4.1, 3.9; HRDARTMS $m / z$ calcd for $\mathrm{C}_{25} \mathrm{H}_{51} \mathrm{O}_{6} \mathrm{Si}_{2}\left(\mathrm{MH}^{+}\right)$ 503.3219, found 503.3221.

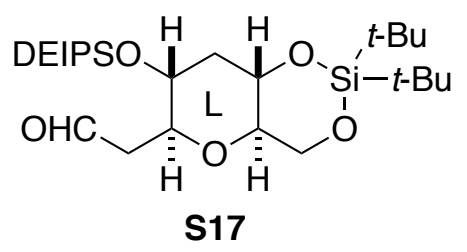

Aldehyde S17. To a solution of ester $\mathbf{S 1 6}\left(2.57 \mathrm{~g}, 5.10 \mathrm{mmol}, 1.0\right.$ equiv) in $\mathrm{CH}_{2} \mathrm{Cl}_{2}(50 \mathrm{~mL})$ at $-80{ }^{\circ} \mathrm{C}$ was added DIBALH (0.94 M solution in $n$-hexane, $5.50 \mathrm{~mL}, 5.20 \mathrm{mmol}, 1.02$ equiv). The reaction mixture was stirred at $-80^{\circ} \mathrm{C}$ for $1.5 \mathrm{~h}$, and the reaction was quenched with $\mathrm{MeOH}(1.6 \mathrm{~mL})$. After warming to room temperature, the mixture was diluted with EtOAc $(60 \mathrm{~mL})$, and $\mathrm{H}_{2} \mathrm{O}(\mathrm{ca} .10 \mathrm{~mL})$ was added dropwise. The mixture was stirred until white precipitates formed. A supernatant solution was collected by decantation with the aid of EtOAc, and concentrated under reduced pressure. Flash chromatography on silica gel (10\% EtOAc in $n$-hexane) afforded aldehyde $\mathbf{S 1 7}(2.26 \mathrm{~g}, 97 \%)$ as a colorless oil. $[\alpha]^{24}{ }_{\mathrm{D}}-47.7$ (c 1.25, $\mathrm{CHCl}_{3}$ ); IR (film) 2957, 2935, 2878, 2862, 2726, 1732, 1472, $1097 \mathrm{~cm}^{-1} ;{ }^{1} \mathrm{H} \mathrm{NMR}(600 \mathrm{MHz}$, $\left.\mathrm{CDCl}_{3}\right) \delta 9.75(1 \mathrm{H}, \mathrm{dd}, J=2.9,1.7 \mathrm{~Hz}), 4.09(1 \mathrm{H}, \mathrm{dd}, J=10.3,5.0 \mathrm{~Hz}), 3.74(1 \mathrm{H}, \mathrm{ddd}, J=11.2,9.2,4.4$ Hz), $3.74(1 \mathrm{H}, \mathrm{t}, J=10.3 \mathrm{~Hz}), 3.70(1 \mathrm{H}, \mathrm{td}, J=9.0,3.5 \mathrm{~Hz}), 3.48(1 \mathrm{H}, \mathrm{ddd}, J=10.8,9.0,4.6 \mathrm{~Hz}), 3.34$ (1H, ddd, $J=10.2,9.3,5.0 \mathrm{~Hz}), 2.79(1 \mathrm{H}, \mathrm{ddd}, J=16.2,3.5,1.7 \mathrm{~Hz}), 2.43(1 \mathrm{H}, \mathrm{ddd}, J=16.2,9.0,2.9$ Hz), $2.42(1 \mathrm{H}, \mathrm{dt}, J=11.9,4.5 \mathrm{~Hz}), 1.56(1 \mathrm{H}, \mathrm{q}, J=11.2 \mathrm{~Hz}), 1.04(9 \mathrm{H}, \mathrm{s}), 1.01-0.97(12 \mathrm{H}, \mathrm{m}), 0.99(9 \mathrm{H}$, s), $0.94(1 \mathrm{H}, \mathrm{m}), 0.68-0.62(4 \mathrm{H}, \mathrm{m}) ;{ }^{13} \mathrm{C} \mathrm{NMR}\left(150 \mathrm{MHz}, \mathrm{CDCl}_{3}\right) \delta 200.9,77.8,77.2,72.1,70.0,66.7$, 46.1, 42.5, 27.4, 27.1, 22.6, 19.9, 17.27, 17.26, 12.9, 7.11, 7.06, 4.1, 3.9; HRDARTMS m/z calcd for $\mathrm{C}_{23} \mathrm{H}_{47} \mathrm{O}_{5} \mathrm{Si}_{2}\left(\mathrm{MH}^{+}\right)$459.2957, found 459.2963. 


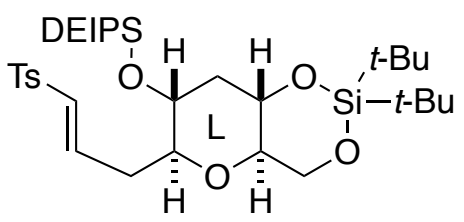

S18

Vinyl sulfone S18. To a solution of aldehyde S17 (2.26 g, $4.92 \mathrm{mmol}, 1.0$ equiv), diethyl ( $p$ toluenesulfonyl)methylphosphonate (1.81 g, $5.90 \mathrm{mmol}, 1.2$ equiv), and $\mathrm{LiCl}$ (250 mg, $5.90 \mathrm{mmol}, 1.2$ equiv) in $\mathrm{CH}_{3} \mathrm{CN}(25 \mathrm{~mL})$ was added $\mathrm{Et}_{3} \mathrm{~N}$ (0.83 mL, $5.90 \mathrm{mmol}, 1.2$ equiv), and the mixture was stirred at room temperature for $3.5 \mathrm{~h}$. The reaction was quenched with saturated $\mathrm{NH}_{4} \mathrm{Cl}$ solution, and the resulting mixture was extracted with EtOAc. The extract was washed with brine, dried, and concentrated under reduced pressure. Flash chromatography on silica gel $(5 \rightarrow 10 \%$ EtOAc in $n$-hexane) afforded vinyl sulfone $\mathbf{S 1 8}(2.80 \mathrm{~g}, 93 \%)$ as a colorless viscous oil. $[\alpha]^{24}{ }_{\mathrm{D}}-24.3\left(c \mathrm{1.03}, \mathrm{CHCl}_{3}\right)$; IR (film) 2956, 2934, 2876, 2861, 1472, 1147, $1093 \mathrm{~cm}^{-1} ;{ }^{1} \mathrm{H}$ NMR $\left(500 \mathrm{MHz}, \mathrm{CDCl}_{3}\right) \delta 7.74(2 \mathrm{H}, \mathrm{d}, J=8.0 \mathrm{~Hz}), 7.32(2 \mathrm{H}, \mathrm{d}, J=$ $8.0 \mathrm{~Hz}), 6.97(1 \mathrm{H}, \mathrm{ddd}, J=15.0,7.3,6.8 \mathrm{~Hz}), 6.33(1 \mathrm{H}, \mathrm{dt}, J=15.0,1.3 \mathrm{~Hz}), 4.04(1 \mathrm{H}, \mathrm{dd}, J=10.1,5.0$ Hz), $3.70(1 \mathrm{H}, \mathrm{t}, J=10.3 \mathrm{~Hz}), 3.69(1 \mathrm{H}, \mathrm{ddd}, J=11.3,9.1,4.3 \mathrm{~Hz}), 3.38(1 \mathrm{H}, \mathrm{ddd}, J=10.8,9.1,4.5 \mathrm{~Hz})$, $3.24(1 \mathrm{H}, \mathrm{ddd}, J=10.3,9.1,5.0 \mathrm{~Hz}), 3.21(1 \mathrm{H}, \mathrm{td}, J=8.8,2.6 \mathrm{~Hz}), 2.72(1 \mathrm{H}, \mathrm{dddd}, J=15.1,6.8,2.6,1.5$ Hz), $2.42(3 \mathrm{H}, \mathrm{s}), 2.37(1 \mathrm{H}, \mathrm{dt}, J=11.9,4.5 \mathrm{~Hz}), 2.24(1 \mathrm{H}, \mathrm{dddd}, J=15.1,8.8,7.3,1.1 \mathrm{~Hz}), 1.49(1 \mathrm{H}$, ddd, $J=11.9,11.3,10.8 \mathrm{~Hz}), 1.03(9 \mathrm{H}, \mathrm{s}), 0.98(9 \mathrm{H}, \mathrm{s}), 0.98-0.87(13 \mathrm{H}, \mathrm{m}), 0.64-0.55(4 \mathrm{H}, \mathrm{m}) ;{ }^{13} \mathrm{C}$ NMR $\left(125 \mathrm{MHz}, \mathrm{CDCl}_{3}\right) \delta 144.2,143.1,137.7,132.3,129.8,127.6,80.6,77.1,72.1,69.8,66.7,42.4$, 33.7, 27.4, 27.1, 22.6, 21.6, 19.9, 17.27, 17.26, 12.9, 7.1, 7.0, 4.1, 3.8; HRDARTMS $\mathrm{m} / z$ calcd for $\mathrm{C}_{31} \mathrm{H}_{55} \mathrm{O}_{6} \mathrm{SSi}_{2}\left(\mathrm{MH}^{+}\right)$611.3252, found 611.3256.

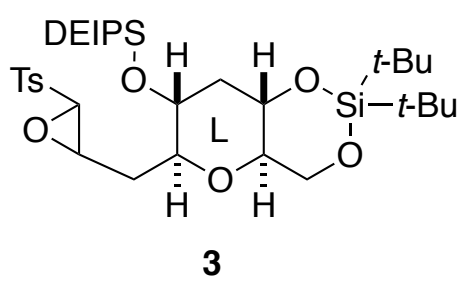


L-ring epoxy sulfone 3. To a solution $t$ - $\mathrm{BuOK}\left(500 \mathrm{mg}, 4.58 \mathrm{mmol}, 1.0\right.$ equiv) in $\mathrm{THF}(40 \mathrm{~mL})$ at $-80{ }^{\circ} \mathrm{C}$ was added $t$-BuOOH (5.5 M solution in nonane, $1.20 \mathrm{~mL}, 6.87 \mathrm{mmol}, 1.5$ equiv), and the mixture was stirred at $-80^{\circ} \mathrm{C}$ for $15 \mathrm{~min}$. A solution of vinyl sulfone $\mathbf{S 1 8}$ (2.80 g, $4.58 \mathrm{mmol}, 1.0$ equiv) in THF (6 $\mathrm{mL}$ ) was added, and the mixture was warmed to $-25^{\circ} \mathrm{C}$. The reaction mixture was stirred at $-25^{\circ} \mathrm{C}$ for 2 h. The reaction was quenched with saturated aqueous $\mathrm{NH}_{4} \mathrm{Cl}$ solution, and the resulting mixture was extracted with EtOAc. The extract was washed with $10 \%$ aqueous $\mathrm{Na}_{2} \mathrm{~S}_{2} \mathrm{O}_{3}$ solution and brine, dried, and concentrated under reduced pressure. Flash chromatography on silica gel (10\% EtOAc in $n$-hexane) afforded epoxy sulfone $3(2.66 \mathrm{~g}, 93 \%, \mathrm{dr}=85: 15)$ as a colorless oil. $[\alpha]^{25} \mathrm{D}-18.8\left(c 2.37, \mathrm{CHCl}_{3}\right)$; $\mathrm{IR}$ (film) $2957,2935,2877,2862,1154,1090 \mathrm{~cm}^{-1} ;{ }^{1} \mathrm{H}$ NMR for the major isomer $\left(600 \mathrm{MHz}, \mathrm{CDCl}_{3}\right) \delta$ $7.80(2 \mathrm{H}, \mathrm{d}, J=8.3 \mathrm{~Hz}), 7.38(2 \mathrm{H}, \mathrm{d}, J=8.3 \mathrm{~Hz}), 4.11(1 \mathrm{H}, \mathrm{dd}, J=10.2,4.9 \mathrm{~Hz}), 3.89(1 \mathrm{H}, \mathrm{d}, J=1.7$ Hz), $3.76(1 \mathrm{H}, \mathrm{t}, J=10.3 \mathrm{~Hz}), 3.75(1 \mathrm{H}, \mathrm{td}, J=5.5,1.7 \mathrm{~Hz}), 3.72(1 \mathrm{H}, \mathrm{ddd}, J=11.2,9.2,4.5 \mathrm{~Hz}), 3.43$ (1H, ddd, $J=10.8,9.0,4.6 \mathrm{~Hz}), 3.25(1 \mathrm{H}, \mathrm{ddd}, J=10.1,9.3,5.0 \mathrm{~Hz}), 3.22(1 \mathrm{H}, \mathrm{td}, J=8.8,2.6 \mathrm{~Hz}), 2.46$ $(3 \mathrm{H}, \mathrm{s}), 2.38(1 \mathrm{H}, \mathrm{dt}, J=11.9,4.4 \mathrm{~Hz}), 2.09(1 \mathrm{H}, \mathrm{ddd}, J=14.7,5.6,2.6 \mathrm{~Hz}), 1.78(1 \mathrm{H}, \mathrm{ddd}, J=14.7,8.9$, $5.3 \mathrm{~Hz}), 1.48(1 \mathrm{H}, \mathrm{q}, J=11.2 \mathrm{~Hz}), 1.04(9 \mathrm{H}, \mathrm{s}), 0.99(9 \mathrm{H}, \mathrm{s}), 0.98-0.87(13 \mathrm{H}, \mathrm{m}), 0.65-0.59(4 \mathrm{H}, \mathrm{m}) ;{ }^{1} \mathrm{H}$ NMR for the minor isomer $\left(600 \mathrm{MHz}, \mathrm{CDCl}_{3}\right) \delta 7.80(2 \mathrm{H}, \mathrm{d}, J=8.3 \mathrm{~Hz}), 7.38(2 \mathrm{H}, \mathrm{d}, J=8.3 \mathrm{~Hz}), 4.07$ $(1 \mathrm{H}, \mathrm{dd}, J=10.2,5.0 \mathrm{~Hz}), 3.88(1 \mathrm{H}, \mathrm{d}, J=1.7 \mathrm{~Hz}), 3.78(1 \mathrm{H}, \mathrm{ddd}, J=7.0,5.1,1.6 \mathrm{~Hz}), 3.76-3.69(2 \mathrm{H}$, m), $3.38(1 \mathrm{H}, \mathrm{ddd}, J=10.8,8.9,4.5 \mathrm{~Hz}), 3.28-3.20(2 \mathrm{H}, \mathrm{m}), 2.46(3 \mathrm{H}, \mathrm{s}), 2.38(1 \mathrm{H}, \mathrm{m}), 2.07(1 \mathrm{H}, \mathrm{ddd}, J$ $=14.0,6.8,4.6 \mathrm{~Hz}), 1.59(1 \mathrm{H}, \mathrm{ddd}, J=14.0,10.4,5.1 \mathrm{~Hz}), 1.51(1 \mathrm{H}, \mathrm{q}, J=11.5 \mathrm{~Hz}), 1.04(9 \mathrm{H}, \mathrm{s}), 0.99$ $(9 \mathrm{H}, \mathrm{s}), 0.98-0.87(13 \mathrm{H}, \mathrm{m}), 0.65-0.59(4 \mathrm{H}, \mathrm{m}) ;{ }^{13} \mathrm{C} \mathrm{NMR}$ for the major isomer $\left(150 \mathrm{MHz}, \mathrm{CDCl}_{3}\right) \delta$ $145.6,134.1,130.0,128.7,79.5,77.0,72.1,69.7,68.0,66.7,55.7,42.4,32.4,27.4,27.1,22.6,21.7,19.9$, $17.3(\mathrm{x} 2), 12.9,7.10,7.06,4.1,3.8 ;{ }^{13} \mathrm{C} \mathrm{NMR}$ for the minor isomer $\left(150 \mathrm{MHz}, \mathrm{CDCl}_{3}\right) \delta 145.6,134.2$, $130.0,128.8,79.0,77.1,72.2,70.2,69.0,66.6,55.4,42.5,33.4,27.4,27.1,22.6,21.7,19.9,17.3(x 2)$, 12.9, 7.10, 7.06, 4.1, 3.8; HRDARTMS $m / z$ calcd for $\mathrm{C}_{31} \mathrm{H}_{55} \mathrm{O}_{7} \mathrm{SSi}_{2}\left(\mathrm{MH}^{+}\right)$627.3202, found 627.3198. 


\section{Synthesis of the GHIJKL fragment 1 (Scheme 6)}

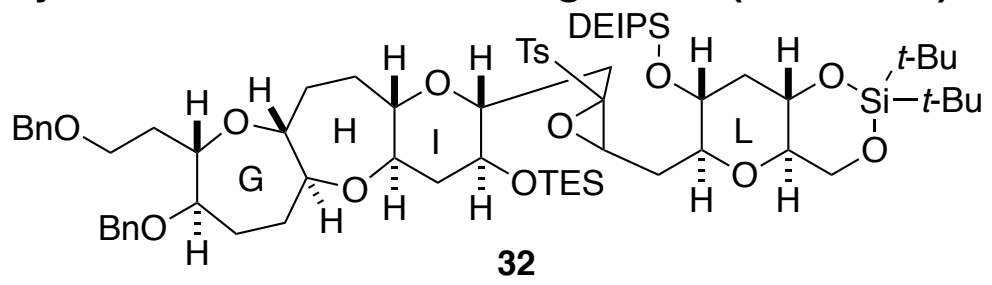

Coupling product 32. (i) Synthesis of triflate 4: To a solution of diol 21 (229 mg, $0.446 \mathrm{mmol}, 1.0$ equiv) in $\mathrm{CH}_{2} \mathrm{Cl}_{2}(4.5 \mathrm{~mL})$ and 2,6-lutidine $\left(156 \mu \mathrm{L}, 1.35 \mathrm{mmol}, 3.0\right.$ equiv) at $-80{ }^{\circ} \mathrm{C}$ was added $\mathrm{Tf}_{2} \mathrm{O}$ ( $77.0 \mu \mathrm{L}, 0.458 \mathrm{mmol}, 1.03$ equiv), and the reaction mixture was stirred at $-80{ }^{\circ} \mathrm{C}$ for $0.5 \mathrm{~h}$. After addition of TESOTf $\left(111 \mu \mathrm{L}, 0.491 \mathrm{mmol}, 1.1\right.$ equiv), the mixture was stirred at $-80{ }^{\circ} \mathrm{C}$ for $1.5 \mathrm{~h}$. The reaction was quenched with saturated aqueous $\mathrm{NaHCO}_{3}$ solution, and the resulting mixture was extracted with EtOAc. The extract was washed with water and brine, dried, and concentrated under reduced pressure. Flash chromatography on silica gel (10\% EtOAc in $n$-hexane) afforded alkyl triflate 4 (305 $\mathrm{mg}, 89 \%)$ as a colorless oil which was immediately used in the next step.

(ii) Oxiranyl anion coupling of alkyl triflate 4 and epoxy sulfone 3. To a solution of alkyl triflate 4 (305 mg, $0.402 \mathrm{mmol}, 1.0$ equiv) and epoxy sulfone 3 (85:15 dr, $376 \mathrm{mg}, 0.60 \mathrm{mmol}, 1.5$ equiv) in THF (4 $\mathrm{mL})$ and HMPA $\left(209 \mu \mathrm{L}, 1.20 \mathrm{mmol}, 3.0\right.$ equiv) at $-100{ }^{\circ} \mathrm{C}$ was added $n$-BuLi $(0.58 \mathrm{mmol}, 0.37 \mathrm{~mL}$, 1.45 equiv). The reaction mixture was stirred at $-100{ }^{\circ} \mathrm{C}$ for $1.5 \mathrm{~h}$, and the reaction was quenched with saturated aqueous $\mathrm{NH}_{4} \mathrm{Cl}$ solution. The resulting mixture was extracted with EtOAc, and the extract was washed with brine, dried, and concentrated under reduced pressure. Flash chromatography on silica gel (20\% $\mathrm{Et}_{2} \mathrm{O}$ in $n$-hexane) afforded the coupling product 32 (477 $\mathrm{mg}, 96 \%$, single diastereomer) and the recovered epoxy sulfone $\mathbf{3}$ (71 $\mathrm{mg}, 53 \%$ recovery based on consumed $\mathbf{3}, \mathrm{dr}=79: 21)$.

Coupling product 32. Colorless amorphous solid; $[\alpha]^{22} \mathrm{D}-15.7$ (c 1.27, $\mathrm{CHCl}_{3}$ ); IR (film) 2936, 2875, $1089 \mathrm{~cm}^{-1} ;{ }^{1} \mathrm{H}$ NMR (600 MHz, $\left.\mathrm{CDCl}_{3}\right) \delta 7.82(2 \mathrm{H}, \mathrm{d}, J=8.3 \mathrm{~Hz}), 7.35-7.25(12 \mathrm{H}, \mathrm{m}), 4.54$ and 4.36 (each $1 \mathrm{H}, \mathrm{d}, J=11.7 \mathrm{~Hz}), 4.51$ and $4.45($ each $1 \mathrm{H}, \mathrm{d}, J=12.1 \mathrm{~Hz}), 4.16(1 \mathrm{H}, \mathrm{dd}, J=10.2,4.9 \mathrm{~Hz}), 3.88$ $(1 \mathrm{H}, \mathrm{dd}, J=6.6,5.5 \mathrm{~Hz}), 3.78(1 \mathrm{H}, \mathrm{t}, J=10.2 \mathrm{~Hz}), 3.73(1 \mathrm{H}, \mathrm{ddd}, J=11.1,9.2,4.4 \mathrm{~Hz}), 3.60(1 \mathrm{H}, \mathrm{ddd}$, $J=9.4,5.0,3.8 \mathrm{~Hz}), 3.54(1 \mathrm{H}, \mathrm{ddd}, J=9.2,8.0,6.2 \mathrm{~Hz}), 3.51(1 \mathrm{H}, \mathrm{ddd}, J=9.2,7.3,5.0 \mathrm{~Hz}), 3.45(1 \mathrm{H}$, 
ddd, $J=10.8,8.9,4.5 \mathrm{~Hz}), 3.37(1 \mathrm{H}, \mathrm{dt}, J=9.0,6.0 \mathrm{~Hz}), 3.70(1 \mathrm{H}, \mathrm{td}, J=4.6,3.7 \mathrm{~Hz}), 3.26(1 \mathrm{H}, \mathrm{ddd}, J$ $=10.1,9.0,4.8 \mathrm{~Hz}), 3.24(1 \mathrm{H}, \mathrm{td}, J=9.0,4.6 \mathrm{~Hz}), 3.16(1 \mathrm{H}, \mathrm{td}, J=8.9,2.8 \mathrm{~Hz}), 3.12(1 \mathrm{H}, \mathrm{ddd}, J=10.7$, 9.0, $4.7 \mathrm{~Hz}), 2.99(1 \mathrm{H}, \mathrm{ddd}, J=10.1,9.0,1.1 \mathrm{~Hz}), 2.96(1 \mathrm{H}, \mathrm{ddd}, J=11.6,9.0,4.0 \mathrm{~Hz}), 2.86(1 \mathrm{H}, \mathrm{dd}, J$ $=15.5,1.2 \mathrm{~Hz}), 2.52(1 \mathrm{H}, \mathrm{td}, J=8.3,5.0 \mathrm{~Hz}), 2.43(3 \mathrm{H}, \mathrm{s}), 2.39(1 \mathrm{H}, \mathrm{dt}, J=11.9,4.4 \mathrm{~Hz}), 2.15(1 \mathrm{H}, \mathrm{dt}$, $J=11.7,4.3 \mathrm{~Hz}), 2.04(1 \mathrm{H}, \mathrm{ddd}, J=14.7,6.8,2.8 \mathrm{~Hz}), 1.93-1.82(3 \mathrm{H}, \mathrm{m}), 1.80-1.53(9 \mathrm{H}, \mathrm{m}), 1.48(1 \mathrm{H}$, q, $J=11.3 \mathrm{~Hz}), 1.30(1 \mathrm{H}, \mathrm{q}, J=11.6 \mathrm{~Hz}), 1.05(9 \mathrm{H}, \mathrm{s}), 1.00(9 \mathrm{H}, \mathrm{s}), 0.99-0.94(22 \mathrm{H}, \mathrm{m}), 0.67-0.58(10 \mathrm{H}$, $\mathrm{m}) ;{ }^{13} \mathrm{C} \mathrm{NMR}\left(150 \mathrm{MHz}, \mathrm{CDCl}_{3}\right) \delta 144.8,138.51,138.45,134.7,129.5(\mathrm{x} 2), 128.35,128.31,127.7$ $127.57,127.56,127.5,83.7,83.4,82.1,81.3,81.2,80.1,79.8,78.3,77.1,74.3,73.0,72.1,70.8,70.7,69.9$, $67.1,66.8,57.7,42.5,41.0,34.9,30.14,30.07,28.2,28.0(x 2), 27.4,27.1,23.9,22.6,21.7,19.9,17.5$ 17.4, 12.9, 7.25, 7.16, 6.9, 5.0, 4.1, 3.9; HRDARTMS m/z calcd for $\mathrm{C}_{67} \mathrm{H}_{107} \mathrm{O}_{13} \mathrm{SSi}_{3}\left(\mathrm{MH}^{+}\right)$1235.6735, found 1235.6727 .

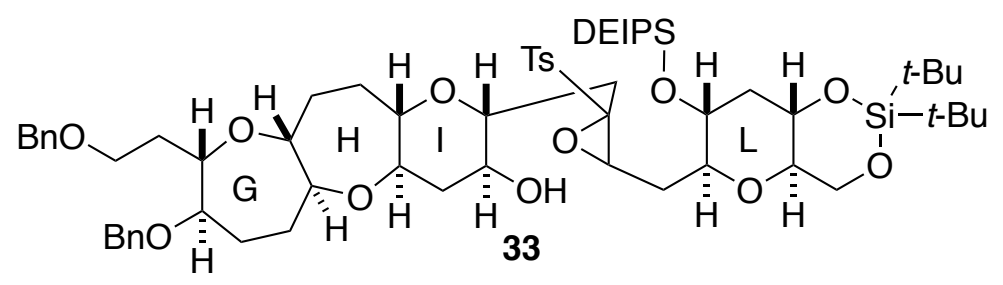

Epoxy alcohol 33. To a solution of the coupling product 32 (472 mg, $0.382 \mathrm{mmol})$ in $\mathrm{EtOH}-\mathrm{CH}_{2} \mathrm{Cl}_{2}$ $(1: 1)(7.6 \mathrm{~mL})$ at $0{ }^{\circ} \mathrm{C}$ was added CSA $(20.0 \mathrm{mg}, 0.0861 \mathrm{mmol}, 0.23 \mathrm{mmol})$, and the reaction mixture was stirred at $0{ }^{\circ} \mathrm{C}$ for $3.5 \mathrm{~h}$. The reaction was quenched with $\mathrm{Et}_{3} \mathrm{~N}$, and the resulting mixture was concentrated under reduced pressure. Flash chromatography on silica gel (10 $\rightarrow 40 \%$ EtOAc in $n$-hexane) afforded epoxy alcohol 33 (191 mg, 46\%) and the recovered coupling product 32 (160 mg, 35\%). The recovered 32 was subjected to the additional two-cycle desilylation. After the three cycles of the reaction, epoxy alcohol 33 (320 mg, 75\%) was obtained as a colorless amorphous solid. $[\alpha]^{22} \mathrm{D}-44.0\left(c 0.99, \mathrm{CHCl}_{3}\right)$; IR (film) 3524, 2935, 2862, 1089, $1061 \mathrm{~cm}^{-1} ;{ }^{1} \mathrm{H} \mathrm{NMR}\left(600 \mathrm{MHz}, \mathrm{CDCl}_{3}\right) \delta 7.80(2 \mathrm{H}, \mathrm{d}, J=8.3 \mathrm{~Hz}), 7.36-$ $7.25(12 \mathrm{H}, \mathrm{m}), 4.54$ and $4.36($ each $1 \mathrm{H}, \mathrm{d}, J=11.7 \mathrm{~Hz}), 4.51$ and $4.45($ each $1 \mathrm{H}, \mathrm{d}, J=11.9 \mathrm{~Hz}), 4.14(1 \mathrm{H}$, dd, $J=10.2,4.9 \mathrm{~Hz}), 3.78(1 \mathrm{H}, \mathrm{dd}, J=7.3,5.3 \mathrm{~Hz}), 3.78(1 \mathrm{H}, \mathrm{t}, J=10.3 \mathrm{~Hz}), 3.72(1 \mathrm{H}, \mathrm{ddd}, J=11.2$, 9.2, 4.4 Hz), $3.63(1 \mathrm{H}, \mathrm{ddd}, J=9.4,4.7,3.9 \mathrm{~Hz}), 3.55(1 \mathrm{H}, \mathrm{ddd}, J=9.4,8.3,6.1 \mathrm{~Hz}), 3.52(1 \mathrm{H}, \mathrm{ddd}, J=$ 
9.3, 7.0, $5.0 \mathrm{~Hz}), 3.43(1 \mathrm{H}, \mathrm{ddd}, J=11.0,9.2,4.8 \mathrm{~Hz}), 3.42(1 \mathrm{H}, \mathrm{dt}, J=9.0,6.0 \mathrm{~Hz}), 3.39(1 \mathrm{H}, \mathrm{ddd}, J=$ 5.8, 5.0, $2.6 \mathrm{~Hz}), 3.30(1 \mathrm{H}, \mathrm{ddd}, J=9.4,7.5,3.5 \mathrm{~Hz}), 3.27(1 \mathrm{H}, \mathrm{td}, J=9.2,4.6 \mathrm{~Hz}), 3.21(1 \mathrm{H}, \mathrm{ddd}, J=$ 10.0, 9.4, $5.0 \mathrm{~Hz}), 3.20(1 \mathrm{H}, \mathrm{ddd}, J=11.0,9.4,7.9,4.7 \mathrm{~Hz}), 3.11(1 \mathrm{H}, \mathrm{td}, J=8.9,2.7 \mathrm{~Hz}), 3.05(1 \mathrm{H}, \mathrm{ddd}$, $J=11.3,9.1,4.1 \mathrm{~Hz}), 2.80(1 \mathrm{H}, \mathrm{td}, J=8.6,3.9 \mathrm{~Hz}), 2.59(1 \mathrm{H}, \mathrm{dd}, J=15.9,3.6 \mathrm{~Hz}), 2.44(3 \mathrm{H}, \mathrm{s}), 2.37$ $(1 \mathrm{H}, \mathrm{dt}, J=11.9,4.4 \mathrm{~Hz}), 2.34(1 \mathrm{H}, \mathrm{dt}, J=11.6,4.4 \mathrm{~Hz}), 2.01(1 \mathrm{H}, \mathrm{ddd}, J=14.5,7.3,2.6 \mathrm{~Hz}), 1.97(1 \mathrm{H}$ $\mathrm{d}, J=7.9 \mathrm{~Hz}), 1.93(1 \mathrm{H}, \mathrm{dddd}, J=15.0,7.5,6.2,1.4 \mathrm{~Hz}), 1.89-1.76(6 \mathrm{H}, \mathrm{m}), 1.73-1.57(5 \mathrm{H}, \mathrm{m}), 1.46$ $(1 \mathrm{H}, \mathrm{q}, J=11.2 \mathrm{~Hz}), 1.36(1 \mathrm{H}, \mathrm{q}, J=11.4 \mathrm{~Hz}), 1.05(9 \mathrm{H}, \mathrm{s}), 1.01(9 \mathrm{H}, \mathrm{s}), 1.00-0.89(13 \mathrm{H}, \mathrm{m}), 0.66-0.59$ $(4 \mathrm{H}, \mathrm{m}) ;{ }^{13} \mathrm{C} \mathrm{NMR}\left(150 \mathrm{MHz}, \mathrm{CDCl}_{3}\right) \delta 145.4,138.5,138.4,133.5,129.7,129.5,128.32,128.3,127.65$ $127.57,127.53,127.50,84.0,83.4,82.0,81.6,81.1,80.0,79.8,78.3,77.1,74.2,72.9,72.1,70.7,70.2$, $69.9,67.1,66.8,58.6,42.4,41.0,34.9,30.2,30.0,28.5,28.2,28.0,27.4,27.1,23.8,22.6,21.7,19.9,17.4$ 17.3, 12.9, 7.2, 7.1, 4.1, 3.8; HRDARTMS $m / z$ calcd for $\mathrm{C}_{61} \mathrm{H}_{96} \mathrm{NO}_{13} \mathrm{SSi}_{2}\left(\mathrm{M}+\mathrm{NH}_{4}^{+}\right)$1138.6135, found 1138.6139.

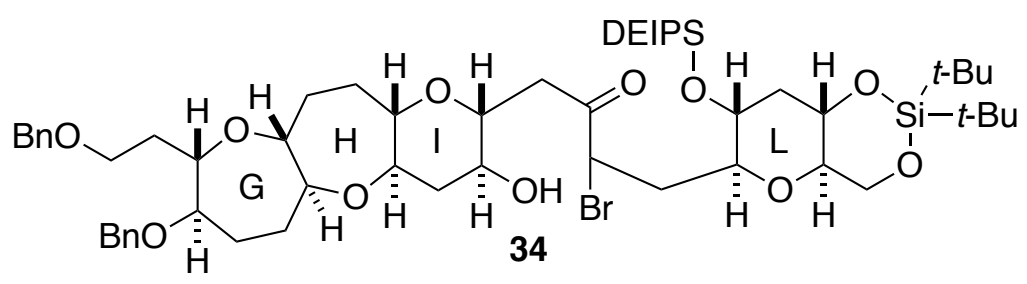

Bromoketone 34. To a solution of epoxy alcohol 33 (308 mg, $0.275 \mathrm{mmol})$ and $\mathrm{LiBr}(179 \mathrm{mg}, 2.06$ mmol) in $\mathrm{CH}_{2} \mathrm{Cl}_{2}(5.5 \mathrm{~mL})$ at $-15{ }^{\circ} \mathrm{C}$ was added $\mathrm{MgBr}_{2} \cdot \mathrm{OEt}_{2}(213 \mathrm{mg}, 0.825 \mathrm{mmol})$. The reaction mixture was stirred at $-15^{\circ} \mathrm{C}$ for $3 \mathrm{~h}$ and then at $0{ }^{\circ} \mathrm{C}$ for $16 \mathrm{~h}$. The reaction was quenched with saturated aqueous $\mathrm{NaHCO}_{3}$ solution, and the resulting mixture was extracted with EtOAc. The extract was washed with brine, dried, and concentrated under reduced pressure. Flash chromatography on silica gel (25 $\rightarrow 30 \%$ EtOAc in $n$-hexane) afforded bromoketone $34(259 \mathrm{mg}, 91 \%)$ as a colorless amorphous solid. $[\alpha]^{24} \mathrm{D}-$ 67.9 (c 1.54, $\mathrm{CHCl}_{3}$ ); IR (film) 3444, 2935, 2862, 1716, 1093, $1061 \mathrm{~cm}^{-1} ;{ }^{1} \mathrm{H} \mathrm{NMR}\left(600 \mathrm{MHz}, \mathrm{CDCl}_{3}\right) \delta$ 7.35-7.25 (10H, m), $4.55(1 \mathrm{H}, \mathrm{dd}, J=10.1,2.8 \mathrm{~Hz}), 4.54$ and 4.36 (each $1 \mathrm{H}, \mathrm{d}, J=11.7 \mathrm{~Hz}), 4.51$ and $4.44($ each $1 \mathrm{H}, \mathrm{d}, J=12.1 \mathrm{~Hz}), 4.10(1 \mathrm{H}, \mathrm{dd}, J=10.1,5.0 \mathrm{~Hz}), 3.72(1 \mathrm{H}, \mathrm{t}, J=10.3 \mathrm{~Hz}), 3.71(1 \mathrm{H}, \mathrm{ddd}$, 
$J=11.4,9.0,4.4 \mathrm{~Hz}), 3.64(1 \mathrm{H}, \mathrm{ddd}, J=9.4,4.6,3.9 \mathrm{~Hz}), 3.57-3.49$ (3H, m), 3.47-3.42 (2H, m), 3.41-

$3.37(2 \mathrm{H}, \mathrm{m}), 3.35(1 \mathrm{H}, \mathrm{ddd}, J=10.6,8.8,1.8 \mathrm{~Hz}), 3.31(1 \mathrm{H}, \mathrm{td}, J=9.0,4.4 \mathrm{~Hz}), 3.27(1 \mathrm{H}, \mathrm{ddd}, J=10.0$ 9.0, $5.0 \mathrm{~Hz}), 3.15(1 \mathrm{H}, \mathrm{ddd}, J=11.4,9.0,4.4 \mathrm{~Hz}), 3.05(1 \mathrm{H}, \mathrm{dd}, J=16.0,4.6 \mathrm{~Hz}), 3.04(1 \mathrm{H}, \mathrm{m}), 2.88(1 \mathrm{H}$, $\mathrm{dd}, J=16.0,6.8 \mathrm{~Hz}), 2.44(1 \mathrm{H}, \mathrm{ddd}, J=15.0,10.3,1.8 \mathrm{~Hz}), 2.39(1 \mathrm{H}, \mathrm{dt}, J=11.9,4.6 \mathrm{~Hz}), 2.37(1 \mathrm{H}, \mathrm{dt}$, $J=11.4,4.2 \mathrm{~Hz}), 1.96-1.78(8 \mathrm{H}, \mathrm{m}), 1.75-1.59(4 \mathrm{H}, \mathrm{m}), 1.55(1 \mathrm{H}, \mathrm{q}, J=11.4 \mathrm{~Hz}), 1.46(1 \mathrm{H}, \mathrm{q}, J=11.4$ $\mathrm{Hz}), 1.03(9 \mathrm{H}, \mathrm{s}), 1.03-0.91(13 \mathrm{H}, \mathrm{m}), 0.99(9 \mathrm{H}, \mathrm{s}), 0.68-0.63(4 \mathrm{H}, \mathrm{m}) ;{ }^{13} \mathrm{C} \mathrm{NMR}\left(150 \mathrm{MHz}, \mathrm{CDCl}_{3}\right) \delta$ 202.3, 138.5, 138.4, 128.33, 128.31, 127.7, 127.6, 127.53, 127.50, 84.1, 83.3, 82.0 (x2), 81.0, 79.8, 79.3, 78.2, 77.0, 73.0, 72.2, 70.7, 70.2, 69.9, 67.1, 66.7, 51.0, 42.5, 42.4, 40.8, 35.6, 34.9, 30.2, 28.3, 28.0, 27.4, 27.1, 23.8, 22.6, 19.9, 17.3 (x2), 12.9, 7.13, 7.09, 4.1, 3.8; HRDARTMS $m / z$ calcd for $\mathrm{C}_{54} \mathrm{H}_{86} \mathrm{O}_{11} \mathrm{BrSi}_{2}$ $\left(\mathrm{MH}^{+}\right)$1045.4887, found 1045.4895 .

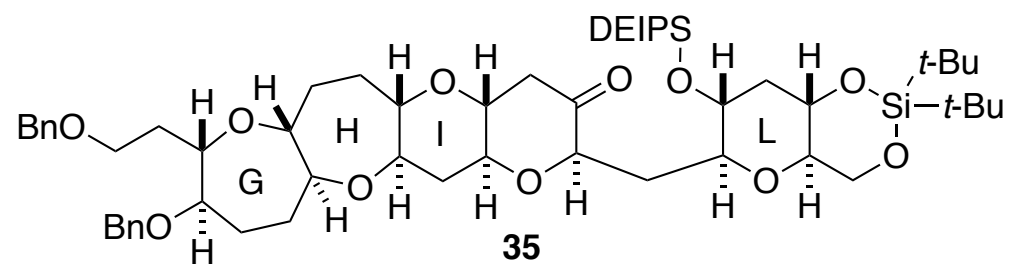

Six-membered ketone 35. To a solution of bromoketone 34 (259 mg, $0.248 \mathrm{mmol})$ in $\mathrm{CH}_{2} \mathrm{Cl}_{2}(2.5 \mathrm{~mL})$ at $0{ }^{\circ} \mathrm{C}$ was added $\mathrm{DBU}\left(41.0 \mu \mathrm{L}, 0.272 \mathrm{mmol}, 1.1\right.$ equiv). The reaction mixture was stirred at $0{ }^{\circ} \mathrm{C}$ for 1 h, and the reaction was quenched with saturated aqueous $\mathrm{NH}_{4} \mathrm{Cl}$ solution. The resulting mixture was extracted with EtOAc, washed with brine, dried, and concentrated under reduced pressure. Flash chromatography on silica gel (20\% EtOAc in $n$-hexane) afforded the six-membered ketone 35 (214 mg, $89 \%$ ) as a colorless amorphous solid. $[\alpha]^{23} \mathrm{D}-45.0\left(c\right.$ 1.32, $\left.\mathrm{CHCl}_{3}\right)$; IR (film) 2935, 2860, 1726, $1093 \mathrm{~cm}^{-}$ 1, ${ }^{1} \mathrm{H}$ NMR (600 MHz, $\left.\mathrm{CDCl}_{3}\right) \delta 7.35-7.26(10 \mathrm{H}, \mathrm{m}), 4.55$ and 4.37 (each $\left.1 \mathrm{H}, \mathrm{d}, J=11.7 \mathrm{~Hz}\right), 4.53$ and $4.45($ each $1 \mathrm{H}, \mathrm{d}, J=12.1 \mathrm{~Hz}), 4.03(1 \mathrm{H}, \mathrm{dd}, J=10.1,5.0 \mathrm{~Hz}), 3.95(1 \mathrm{H}, \mathrm{dd}, J=6.7,3.4 \mathrm{~Hz}), 3.76(1 \mathrm{H}$, t, $J=10.2 \mathrm{~Hz}), 3.70(1 \mathrm{H}, \mathrm{ddd}, J=11.2,9.2,4.4 \mathrm{~Hz}), 3.67(1 \mathrm{H}, \mathrm{dt}, J=9.6,4.0 \mathrm{~Hz}), 3.56(1 \mathrm{H}, \mathrm{ddd}, J=9.2$, 8.1, $5.9 \mathrm{~Hz}), 3.52(1 \mathrm{H}, \mathrm{ddd}, J=9.2,6.8,5.0 \mathrm{~Hz}), 3.49(1 \mathrm{H}, \mathrm{dt}, J=9.2,6.1 \mathrm{~Hz}), 3.44(1 \mathrm{H}, \mathrm{ddd}, J=9.0$, 6.2, 4.6 Hz), $3.42(1 \mathrm{H}, \mathrm{m}), 3.37(1 \mathrm{H}, \mathrm{td}, J=9.4,3.2 \mathrm{~Hz}), 3.34(1 \mathrm{H}, \mathrm{td}, J=9.5,4.8 \mathrm{~Hz}), 3.31-3.26(3 \mathrm{H}$, 
m), $3.20(1 \mathrm{H}, \mathrm{ddd}, J=10.0,9.3,4.9 \mathrm{~Hz}), 3.13(1 \mathrm{H}, \mathrm{ddd}, J=8.9,8.0,4.4 \mathrm{~Hz}), 2.86(1 \mathrm{H}, \mathrm{dd}, J=16.9,5.0$

Hz), $2.41(1 \mathrm{H}, \mathrm{dt}, J=11.4,4.4 \mathrm{~Hz}), 2.40(1 \mathrm{H}, \mathrm{ddd}, J=14.2,6.8,3.5 \mathrm{~Hz}), 2.35(1 \mathrm{H}, \mathrm{dt}, J=11.9,4.4 \mathrm{~Hz})$, $2.33(1 \mathrm{H}, \mathrm{dd}, J=16.9,11.0 \mathrm{~Hz}), 1.95(1 \mathrm{H}, \mathrm{dddd}, J=14.1,8.0,7.0,1.3 \mathrm{~Hz}), 1.92-1.75(8 \mathrm{H}, \mathrm{m}), 1.67(1 \mathrm{H}$, ddt, $J=14.3,12.2,1.9 \mathrm{~Hz}), 1.61(1 \mathrm{H}, \mathrm{dddd}, J=14.0,9.6,5.7,5.1 \mathrm{~Hz}), 1.56(1 \mathrm{H}, \mathrm{q}, J=11.2 \mathrm{~Hz}), 1.50$ $(1 \mathrm{H}, \mathrm{q}, J=11.2 \mathrm{~Hz}), 1.03(9 \mathrm{H}, \mathrm{s}), 1.01-0.89(13 \mathrm{H}, \mathrm{m}), 0.98(9 \mathrm{H}, \mathrm{s}), 0.67-0.60(4 \mathrm{H}, \mathrm{m}) ;{ }^{13} \mathrm{C}$ NMR $(150$ $\left.\mathrm{MHz}, \mathrm{CDCl}_{3}\right) \delta 206.4,138.5,138.4,128.34,128.32,127.7,127.6,127.53,127.51,84.5,83.3,82.0,81.9$, $81.0,79.9,79.5,78.4,77.2,75.8,75.2,73.0,72.3,70.8,70.7,67.1,66.6,44.4,42.7,37.1,35.0,33.0,30.3$, 28.3, 28.0, 27.4, 27.1, 23.8, 22.6, 19.9, 17.3 (x2), 12.9, 7.13, 7.10, 4.1, 3.8; HRDARTMS $m / z$ calcd for $\mathrm{C}_{54} \mathrm{H}_{85} \mathrm{O}_{11} \mathrm{Si}_{2}\left(\mathrm{MH}^{+}\right)$965.5625, found 965.5626.

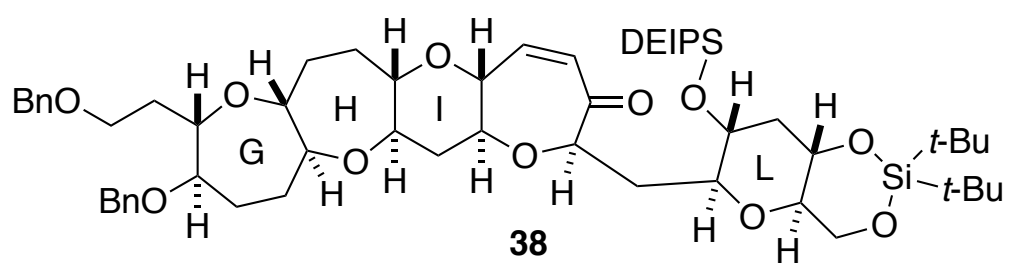

Conjugated ketone 38. (i) Ring expansion reaction with TMS-diazomethane: To a solution of the sixmembered ketone 35 (191 mg, $0.197 \mathrm{mmol})$ and MS4Å (1.0 g, $50 \mathrm{mg}$ per $1 \mathrm{~mL}$ solvent) in $\mathrm{CH}_{2} \mathrm{Cl}_{2}(20$ $\mathrm{mL})$ at $-80{ }^{\circ} \mathrm{C}$ was added $\mathrm{BF}_{3} \cdot \mathrm{OEt}_{2}\left(122 \mu \mathrm{L}, 0.99 \mathrm{mmol}, 5.0\right.$ equiv) and $\mathrm{TMSCHN}_{2}(2.0 \mathrm{M}$ solution in $n$ hexane, $495 \mu \mathrm{L}, 0.99 \mathrm{mmol}, 5.0$ equiv). The reaction mixture was stirred at $-80^{\circ} \mathrm{C}$ for $1 \mathrm{~h}$, and the reaction was quenched with saturated aqueous $\mathrm{NaHCO}_{3}$ solution. The resulting mixture was extracted with EtOAc, washed with brine, dried, and concentrated under reduced pressure to afford $\alpha$-silyl ketone 36 (179 mg) as a colorless amorphous solid, which was used in the next step without purification.

(ii) 1,3-Brook rearrangement: A solution of the $\alpha$-silyl ketone $36(179 \mathrm{mg})$ in benzene $(50 \mathrm{~mL})$ was heated at $140{ }^{\circ} \mathrm{C}$ for $3 \mathrm{~h}$ in a sealed reaction vessel. After cooling to room temperature, the resulting solution was concentrated under reduced pressure to afford silyl enol ether $\mathbf{3 7}(199 \mathrm{mg})$ as a colorless amorphous solid, which was used in the next step without purification. 
(iii) Saegusa oxidation.: To a solution of silyl enol ether $37(199 \mathrm{mg})$ in $\mathrm{CH}_{3} \mathrm{CN}$ (10 mL) was added $\mathrm{Pd}(\mathrm{OAc})_{2}(110 \mathrm{mg}, 0.491 \mathrm{mmol}, 2.5$ equiv), and the reaction mixture was stirred at room temperature for $3 \mathrm{~h}$. The resulting mixture was filtered through a Celite pad, and the filtrate was concentrated under reduced pressure. Flash chromatography on silica gel (20\% EtOAc in $n$-hexane) afforded the conjugated ketone 38 (102 mg, 53\% over three steps) as a colorless amorphous solid. $[\alpha]^{24}-72.1\left(c 0.81, \mathrm{CHCl}_{3}\right)$; IR (film) 2935, 2861, 1669, 1091, $1065 \mathrm{~cm}^{-1} ;{ }^{1} \mathrm{H}$ NMR (600 MHz, $\left.\mathrm{CDCl}_{3}\right) \delta$ 7.35-7.25 (10H, m), 6.35 $(1 \mathrm{H}, \mathrm{dd}, J=12.7,2.3 \mathrm{~Hz}), 5.91(1 \mathrm{H}, \mathrm{dd}, J=12.7,2.6 \mathrm{~Hz}), 4.55$ and $4.37($ each $1 \mathrm{H}, \mathrm{d}, J=11.7 \mathrm{~Hz}), 4.52$ and 4.45 (each $1 \mathrm{H}, \mathrm{d}, J=11.9 \mathrm{~Hz}), 4.28(1 \mathrm{H}, \mathrm{dd}, J=6.1,3.4 \mathrm{~Hz}), 4.01(1 \mathrm{H}, \mathrm{dd}, J=10.1,5.0 \mathrm{~Hz}), 3.85$ $(1 \mathrm{H}, \mathrm{dt}, J=8.9,2.4 \mathrm{~Hz}), 3.70(1 \mathrm{H}, \mathrm{t}, J=10.3 \mathrm{~Hz}), 3.68(1 \mathrm{H}, \mathrm{ddd}, J=11.2,9.0,4.4 \mathrm{~Hz}), 3.66(1 \mathrm{H}, \mathrm{dt}, J=$ 9.2, $4.0 \mathrm{~Hz}), 3.56(1 \mathrm{H}, \mathrm{ddd}, J=9.4,8.3,5.9 \mathrm{~Hz}), 3.52(1 \mathrm{H}, \mathrm{ddd}, J=9.4,6.6,4.8 \mathrm{~Hz}), 3.47(1 \mathrm{H}, \mathrm{dt}, J=$ 9.0, $5.8 \mathrm{~Hz}), 3.43-3.38(3 \mathrm{H}, \mathrm{m}), 3.37(1 \mathrm{H}, \mathrm{ddd}, J=11.2,9.0,4.8 \mathrm{~Hz}), 3.32(1 \mathrm{H}, \mathrm{td}, J=9.2,4.4 \mathrm{~Hz}), 3.20$ $(1 \mathrm{H}, \mathrm{ddd}, J=11.3,9.0,3.9 \mathrm{~Hz}), 3.16(1 \mathrm{H}, \mathrm{ddd}, J=8.8,7.8,4.4 \mathrm{~Hz}), 3.16(1 \mathrm{H}, \mathrm{ddd}, J=10.2,9.0,5.0 \mathrm{~Hz})$, $2.40(1 \mathrm{H}, \mathrm{dt}, J=11.9,4.3 \mathrm{~Hz}), 2.35(1 \mathrm{H}, \mathrm{ddd}, J=14.0,6.1,2.3 \mathrm{~Hz}), 2.33(1 \mathrm{H}, \mathrm{dt}, J=11.5,4.0 \mathrm{~Hz}), 1.96$ $(1 \mathrm{H}, \mathrm{dddd}, J=14.6,7.6,6.1,1.5 \mathrm{~Hz}), 1.91-1.75(8 \mathrm{H}, \mathrm{m}), 1.69-1.57(3 \mathrm{H}, \mathrm{m}), 1.49(1 \mathrm{H}, \mathrm{q}, J=11.4 \mathrm{~Hz})$, $1.02(9 \mathrm{H}, \mathrm{m}), 1.00-0.90(13 \mathrm{H}, \mathrm{m}), 0.98(9 \mathrm{H}, \mathrm{m}), 0.66-0.61(4 \mathrm{H}, \mathrm{m}),{ }^{13} \mathrm{C} \mathrm{NMR}\left(150 \mathrm{MHz}, \mathrm{CDCl}_{3}\right) \delta 204.0$, $141.6,138.5,138.4,128.8,128.3$ (x2), 127.7, 127.6, 127.5 (x2), 84.4, 83.5, 83.3, 82.4, 82.0, 81.0, 79.9, $79.7,78.0,77.4,77.1,72.9,72.2,70.7,70.3,67.1,66.4,42.7,38.2,36.5,34.9,30.2,28.3,28.0,27.5,27.1$, 23.7, 22.6, 19.9, 17.3 (x2), 12.9, 7.11, 7.06, 4.1, 3.8; HRDARTMS $m / z$ calcd for $\mathrm{C}_{55} \mathrm{H}_{85} \mathrm{O}_{11} \mathrm{Si}_{2}\left(\mathrm{MH}^{+}\right)$ 977.5625, found 977.5634 .

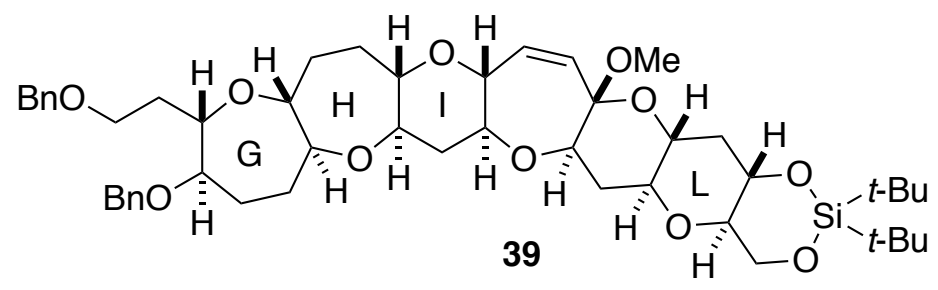

Methyl acetal 39. To a solution of the conjugated ketone $\mathbf{3 8}(89.0 \mathrm{mg}, 0.0911 \mathrm{mmol})$ in a mixed solvent of $\mathrm{CH}_{2} \mathrm{Cl}_{2}-\mathrm{MeOH}-\mathrm{CH}(\mathrm{OMe})_{3}(10: 2: 10)(3 \mathrm{~mL})$ was added $p-\mathrm{TsOH} \cdot \mathrm{H}_{2} \mathrm{O}(11 \mathrm{mg}, 0.058 \mathrm{mmol}, 0.64 \mathrm{mmol})$. 
The reaction mixture was stirred at room temperature for $3 \mathrm{~h}$, and the reaction was quenched with $\mathrm{Et}_{3} \mathrm{~N}$. The resulting mixture was concentrated under reduced pressure. Flash chromatography on silica gel $(20 \%$ EtOAc in $n$-hexane) afforded methyl acetal $\mathbf{3 9}(63.2 \mathrm{mg}, 80 \%)$ as a colorless amorphous solid. $[\alpha]^{24} \mathrm{D}-$ 24.4 (c 0.50, $\left.\mathrm{CHCl}_{3}\right)$; IR (film) 2934, 2859, 1085, 1061, $1040 \mathrm{~cm}^{-1} ;{ }^{1} \mathrm{H}$ NMR $\left(600 \mathrm{MHz}, \mathrm{CDCl}_{3}\right) \delta 7.35-$ $7.25(10 \mathrm{H}, \mathrm{m}), 5.82(1 \mathrm{H}, \mathrm{dd}, J=12.5,2.2 \mathrm{~Hz}), 5.60(1 \mathrm{H}, \mathrm{dd}, J=12.5,2.8 \mathrm{~Hz}), 4.54$ and 4.35 (each 1H, d, $J=11.7 \mathrm{~Hz}), 4.52$ and 4.44 (each 1H, d, $J=11.9 \mathrm{~Hz}), 4.13(1 \mathrm{H}, \mathrm{dd}, J=10.2,4.9 \mathrm{~Hz}), 4.02(1 \mathrm{H}, \mathrm{dt}, J$ $=9.2,2.5 \mathrm{~Hz}), 3.85(1 \mathrm{H}, \mathrm{ddd}, J=11.0,9.2,4.4 \mathrm{~Hz}), 3.82(1 \mathrm{H}, \mathrm{t}, J=10.3 \mathrm{~Hz}), 3.65(1 \mathrm{H}, \mathrm{dt}, J=9.6,4.2$ Hz), $3.56(1 \mathrm{H}, \mathrm{dd}, J=11.7,4.6 \mathrm{~Hz}), 3.55(1 \mathrm{H}, \mathrm{ddd}, J=9.4,8.3,5.8 \mathrm{~Hz}), 3.52(1 \mathrm{H}, \mathrm{ddd}, J=9.2,6.8,5.0$ Hz), $3.45(1 \mathrm{H}, \mathrm{dt}, J=9.0,5.8 \mathrm{~Hz}), 3.41(1 \mathrm{H}, \mathrm{ddd}, J=5.6,4.6,2.6 \mathrm{~Hz}), 3.37(1 \mathrm{H}, \mathrm{ddd}, J=11.2,9.3,4.9$ Hz), 3.34-3.27 (3H, m), $3.24(3 \mathrm{H}, \mathrm{s}), 3.15(1 \mathrm{H}, \mathrm{ddd}, J=11.7,9.0,4.0 \mathrm{~Hz}), 3.09$ (1H, ddd, $J=11.8,9.3$, $4.2 \mathrm{~Hz}), 3.02(1 \mathrm{H}, \mathrm{ddd}, J=9.0,7.5,4.6 \mathrm{~Hz}), 2.34(1 \mathrm{H}, \mathrm{dt}, J=11.6,4.3 \mathrm{~Hz}), 2.28(1 \mathrm{H}, \mathrm{dt}, J=12.1,4.4$ Hz), $2.02(1 \mathrm{H}, \mathrm{dt}, J=11.6,4.4 \mathrm{~Hz}), 1.94(1 \mathrm{H}, \mathrm{dddd}, J=14.7,7.3,6.1,1.4 \mathrm{~Hz}), 1.91-1.83(4 \mathrm{H}, \mathrm{m}), 1.82-$ $1.73(4 \mathrm{H}, \mathrm{m}), 1.65(1 \mathrm{H}, \mathrm{q}, J=11.4 \mathrm{~Hz}), 1.65(1 \mathrm{H}, \mathrm{m}), 1.61(1 \mathrm{H}, \mathrm{dddd}, J=14.0,9.5,5.9,5.0 \mathrm{~Hz}), 1.51$ $(1 \mathrm{H}, \mathrm{q}, J=11.6 \mathrm{~Hz}), 1.04(9 \mathrm{H}, \mathrm{s}), 0.98(9 \mathrm{H}, \mathrm{s}) ;{ }^{13} \mathrm{C} \mathrm{NMR}\left(150 \mathrm{MHz}, \mathrm{CDCl}_{3}\right) \delta 138.5,138.4,136.4,130.9$, $128.3,128.3,127.7,127.6,127.5$ (x2), $98.8,84.3,83.4,82.0,81.3,81.0,79.7,79.5,79.2,78.9,77.6,76.5$, $72.94,72.85,70.7,67.5,67.1,66.8,49.0,38.6,37.9,35.0,32.6,30.2,28.3,28.0,27.4,27.0,23.8,22.6$, 19.9; HRDARTMS $m / z$ calcd for $\mathrm{C}_{49} \mathrm{H}_{71} \mathrm{O}_{11} \mathrm{Si}\left(\mathrm{MH}^{+}\right)$863.4760, found 863.4761 .

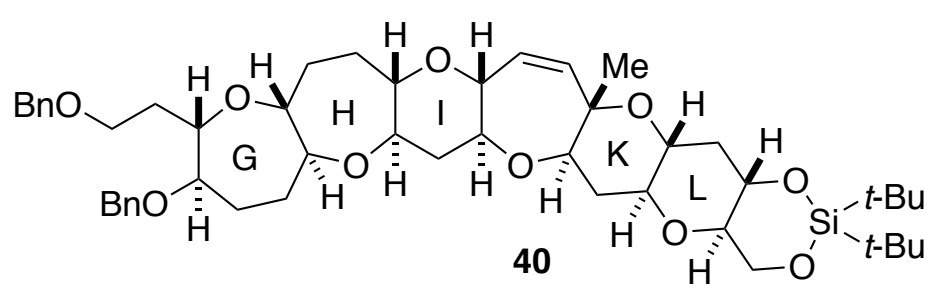

Methylated product 40. To a solution of methyl acetal 39 (44.4 mg, $0.0514 \mathrm{mmol})$ in $\mathrm{CH}_{2} \mathrm{Cl}_{2}(1.0 \mathrm{~mL})$ at $-20^{\circ} \mathrm{C}$ were added $\mathrm{AlMe}_{3}(1.09 \mathrm{M}$ solution in $n$-hexane, $0.41 \mathrm{~mL}, 0.447 \mathrm{mmol}, 8.7$ equiv) and TMSOTf (37.2 $\mu \mathrm{L}, 0.206 \mathrm{mmol}, 4.0$ equiv). The reaction mixture was stirred at $-20^{\circ} \mathrm{C}$ for $6 \mathrm{~h}$, and the reaction was quenched with saturated aqueous $\mathrm{NaHCO}_{3}$ solution. After addition of $10 \%$ aqueous potassium 
sodium tartrate solution, the mixture was extracted with EtOAc. The extract was washed with brine, dried, and concentrated under reduced pressure. Flash chromatography on silica gel (10\% EtOAc in benzene) afforded the methylated product $40(33.2 \mathrm{mg}, 76 \%)$ as a colorless solid; $\mathrm{mp} 177-179{ }^{\circ} \mathrm{C} ;[\alpha]^{25} \mathrm{D}-15.1(c$ 1.09, $\mathrm{CHCl}_{3}$ ); IR (film) 2932, 2859, $1081 \mathrm{~cm}^{-1} ;{ }^{1} \mathrm{H}$ NMR (600 MHz, $\left.\mathrm{CDCl}_{3}\right) \delta 7.35-7.25(10 \mathrm{H}, \mathrm{m}), 5.75$ (1H, dd, $J=12.8,2.8 \mathrm{~Hz}), 5.49(1 \mathrm{H}, \mathrm{dd}, J=12.8,1.8 \mathrm{~Hz}), 4.55$ and $4.36($ each $1 \mathrm{H}, \mathrm{d}, J=11.7 \mathrm{~Hz}), 4.52$ and $4.44($ each $1 \mathrm{H}, \mathrm{d}, J=11.9 \mathrm{~Hz}), 4.13(1 \mathrm{H}, \mathrm{dd}, J=10.1,5.0 \mathrm{~Hz}), 3.84(1 \mathrm{H}, \mathrm{ddd}, J=9.0,2.8,1.8 \mathrm{~Hz})$, $3.83(1 \mathrm{H}, \mathrm{ddd}, J=11.4,9.0,4.5 \mathrm{~Hz}), 3.81(1 \mathrm{H}, \mathrm{t}, J=10.3 \mathrm{~Hz}), 3.64(1 \mathrm{H}, \mathrm{ddd}, J=9.4,5.0,4.0 \mathrm{~Hz}), 3.56$ $(1 \mathrm{H}, \mathrm{ddd}, J=9.2,8.0,6.1 \mathrm{~Hz}), 3.52(1 \mathrm{H}, \mathrm{ddd}, J=9.2,6.8,4.8 \mathrm{~Hz}), 3.50(1 \mathrm{H}, \mathrm{dd}, J=12.1,4.4 \mathrm{~Hz}), 3.44$ (1H, dt, $J=9.0,5.9 \mathrm{~Hz}), 3.40(1 \mathrm{H}, \mathrm{ddd}, J=5.8,5.0,2.4 \mathrm{~Hz}), 3.33-3.29(3 \mathrm{H}, \mathrm{m}), 3.22(1 \mathrm{H}, \mathrm{ddd}, J=11.3$, 9.4, $4.1 \mathrm{~Hz}), 3.17(1 \mathrm{H}, \mathrm{ddd}, J=11.6,9.1,4.1 \mathrm{~Hz}), 3.04$ (1H, ddd, $J=11.5,9.4,4.4 \mathrm{~Hz}), 3.02(1 \mathrm{H}, \mathrm{ddd}, J$ $=9.0,7.5,4.4 \mathrm{~Hz}), 2.32(1 \mathrm{H}, \mathrm{dt}, J=11.7,4.2 \mathrm{~Hz}), 2.29(1 \mathrm{H}, \mathrm{dt}, J=12.1,4.2 \mathrm{~Hz}), 2.05(1 \mathrm{H}, \mathrm{dt}, J=11.9$, $4.2 \mathrm{~Hz}), 1.94(1 \mathrm{H}, \mathrm{dddd}, J=14.7,7.3,5.8,1.5 \mathrm{~Hz}), 1.90-1.83(4 \mathrm{H}, \mathrm{m}), 1.81-1.73(3 \mathrm{H}, \mathrm{m}), 1.66$ (ddt, $J=$ 14.7, 12.0, $1.8 \mathrm{~Hz}), 1.60(1 \mathrm{H}$, dddd, $J=14.0,9.6,5.7,5.1 \mathrm{~Hz}), 1.57(1 \mathrm{H}, \mathrm{q}, J=11.8 \mathrm{~Hz}), 1.54(1 \mathrm{H}, \mathrm{q}, J$ $=11.8 \mathrm{~Hz}), 1.47(1 \mathrm{H}, \mathrm{q}, J=11.3 \mathrm{~Hz}), 1.31(3 \mathrm{H}, \mathrm{s}), 1.03(9 \mathrm{H}, \mathrm{s}), 0.98(9 \mathrm{H}, \mathrm{s}) ;{ }^{13} \mathrm{C} \mathrm{NMR}\left(150 \mathrm{MHz}, \mathrm{CDCl}_{3}\right)$ $\delta 138.8,138.54,138.45,129.3,128.33,128.32,127.7,127.6,127.5(x 2), 84.2,83.6,82.1,81.6,81.1,80.8$, $79.9,79.8,79.7,78.8,77.7,77.4,72.95,72.88,70.7,68.3,67.1,66.8,38.8,38.6,34.9,33.0,30.3,28.4$, 28.0, 27.4, 27.0, 23.8, 22.6, 19.9, 15.5; HRDARTMS $m / z$ calcd for $\mathrm{C}_{49} \mathrm{H}_{71} \mathrm{O}_{10} \mathrm{Si}\left(\mathrm{MH}^{+}\right)$847.4811, found 847.4822 .

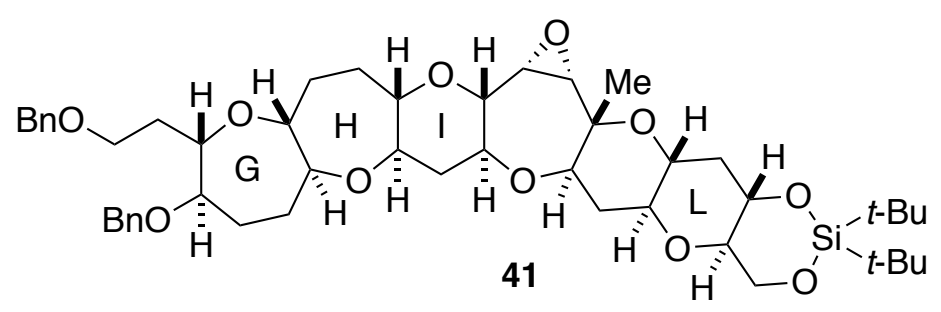

Epoxide 41. To a solution of alkene 40 (36.0 $\mathrm{mg}, 0.0425 \mathrm{mmol})$ in $\mathrm{CH}_{2} \mathrm{Cl}_{2}(0.22 \mathrm{~mL})$ was added $\mathrm{m}$ CPBA (18.9 mg, $0.110 \mathrm{mmol}, 2.6$ equiv), and the reaction mixture was stirred at room temperature for 20 h. Additional $m$-CPBA (9.8 mg, $0.0568 \mathrm{mmol}, 1.3$ equiv) was added and the reaction mixture was stirred 
at room temperature for further $16 \mathrm{~h}$. The reaction was quenched with a 1:1 mixture of saturated aqueous $\mathrm{NaHCO}_{3}$ solution and saturated $\mathrm{Na}_{2} \mathrm{~S}_{2} \mathrm{O}_{3}$ solution, and the resulting mixture was extracted with EtOAc. The extract was washed with another 1:1 mixture of saturated aqueous $\mathrm{NaHCO}_{3}$ solution and saturated $\mathrm{Na}_{2} \mathrm{~S}_{2} \mathrm{O}_{3}$ solution, and the organic layer was washed with brine, dried, and concentrated under reduced pressure. Flash chromatography on silica gel (20\% EtOAc in $n$-hexane) afforded epoxide 41 (32.0 mg, 87\%) as a colorless solid. Mp 183-186 ${ }^{\circ} \mathrm{C} ;[\alpha]^{25} \mathrm{D}-18.2\left(\mathrm{c} 1.02, \mathrm{CHCl}_{3}\right)$; IR (film) 2934, 2859, $1084 \mathrm{~cm}^{-}$ ${ }^{1} ;{ }^{1} \mathrm{H}$ NMR $\left(600 \mathrm{MHz}, \mathrm{CDCl}_{3}\right) \delta 7.35-7.25(10 \mathrm{H}, \mathrm{m}), 4.54$ and 4.36 (each $\left.1 \mathrm{H}, \mathrm{d}, J=11.7 \mathrm{~Hz}\right), 4.52$ and $4.45($ each 1H, d, $J=11.9 \mathrm{~Hz}), 4.13(1 \mathrm{H}, \mathrm{dd}, J=10.2,4.9 \mathrm{~Hz}), 3.83(1 \mathrm{H}, \mathrm{ddd}, J=11.0,9.2,4.6 \mathrm{~Hz}), 3.80$ $(1 \mathrm{H}, \mathrm{t}, J=10.3 \mathrm{~Hz}), 3.64(1 \mathrm{H}, \mathrm{ddd}, J=9.5,4.6,3.9 \mathrm{~Hz}), 3.57(1 \mathrm{H}, \mathrm{dd}, J=12.1,4.4 \mathrm{~Hz}), 3.56(1 \mathrm{H}, \mathrm{ddd}$, $J=9.3,8.2,6.1 \mathrm{~Hz}), 3.52(1 \mathrm{H}, \mathrm{ddd}, J=9.3,6.8,5.0 \mathrm{~Hz}), 3.43(1 \mathrm{H}, \mathrm{dt}, J=9.2,6.2 \mathrm{~Hz}), 3.42-3.38(3 \mathrm{H}$, m), $3.31(1 \mathrm{H}, \mathrm{ddd}, J=10.3,9.2,5.1 \mathrm{~Hz}), 3.30(1 \mathrm{H}, \mathrm{td}, J=9.2,4.2 \mathrm{~Hz}), 3.27(1 \mathrm{H}, \mathrm{d}, J=5.1 \mathrm{~Hz}), 3.24(1 \mathrm{H}$, ddd, $J=11.4,9.4,4.0 \mathrm{~Hz}), 3.19(1 \mathrm{H}, \mathrm{ddd}, J=11.4,9.4,4.0 \mathrm{~Hz}), 3.18(1 \mathrm{H}, \mathrm{d}, J=5.1 \mathrm{~Hz}), 3.06(1 \mathrm{H}, \mathrm{ddd}$, $J=11.7,9.4,4.4 \mathrm{~Hz}), 3.02(1 \mathrm{H}, \mathrm{m}), 2.32(1 \mathrm{H}, \mathrm{dt}, J=11.9,4.2 \mathrm{~Hz}), 2.30(1 \mathrm{H}, \mathrm{dt}, J=11.0,4.0 \mathrm{~Hz}), 2.06$ $(1 \mathrm{H}, \mathrm{dt}, J=11.8,4.3 \mathrm{~Hz}), 1.96-1.75(8 \mathrm{H}, \mathrm{m}), 1.67(1 \mathrm{H}, \mathrm{ddt}, J=14.1,12.5,1.8 \mathrm{~Hz}), 1.60(1 \mathrm{H}, \mathrm{dddd}, J=$ $14.3,10.4,5.5,4.8 \mathrm{~Hz}), 1.56(1 \mathrm{H}, \mathrm{q}, J=12.0 \mathrm{~Hz}), 1.53(1 \mathrm{H}, \mathrm{q}, J=11.4 \mathrm{~Hz}), 1.45(1 \mathrm{H}, \mathrm{q}, J=11.4 \mathrm{~Hz})$, $1.25(3 \mathrm{H}, \mathrm{s}), 1.03(9 \mathrm{H}, \mathrm{s}), 0.98(9 \mathrm{H}, \mathrm{s}) ;{ }^{13} \mathrm{C} \mathrm{NMR}\left(150 \mathrm{MHz}, \mathrm{CDCl}_{3}\right) \delta 138.55,138.45,128.33,128.32$, $127.7,127.6$ (x2), 127.5, 84.2, 83.6, 82.5, 82.1, 81.1, 79.5, 79.2, 77.6, 77.17, 77.15, 75.2, 74.7, 72.94, $72.91,70.7,68.9,67.1,66.8,60.4,56.2,38.4(x 2), 34.9,32.2,30.3,28.3,28.0,27.4,27.0,23.8,22.6,19.9$, 14.4; HRDARTMS $m / z$ calcd for $\mathrm{C}_{49} \mathrm{H}_{71} \mathrm{O}_{11} \mathrm{Si}\left(\mathrm{MH}^{+}\right)$863.4760, found 863.4764.

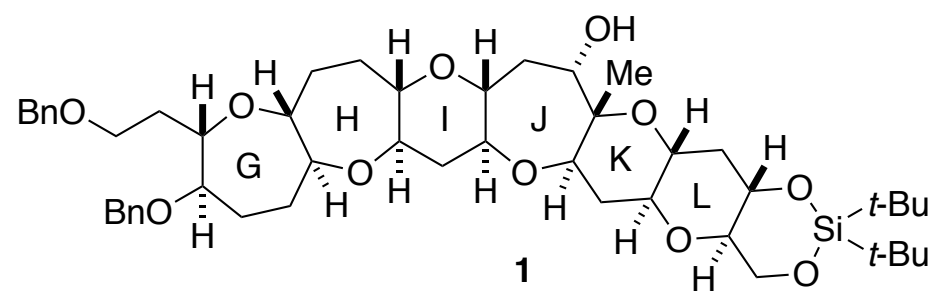

GHIJKL fragment 1. A mixture of $\mathrm{LiBEt}_{3} \mathrm{H}(0.99 \mathrm{M}$ solution in THF, $0.2 \mathrm{~mL}, 0.198 \mathrm{mmol}, 5.4$ equiv) and epoxide 41 (31.9 $\mathrm{mg}, 0.0370 \mathrm{mmol})$ was stirred at room temperature for $2 \mathrm{~h}$. The reaction was 
quenched with $10 \%$ aqueous potassium sodium tartrate solution. The resulting mixture was extracted with EtOAc, washed with brine, dried, and concentrated under reduced pressure. Flash chromatography on silica gel (30\% EtOAc in $n$-hexane) afforded alcohol $1(28.9 \mathrm{mg}, 90 \%)$ as a colorless amorphous solid. $[\alpha]^{25}{ }_{\mathrm{D}}-15.7\left(c\right.$ 2.02, $\left.\mathrm{CHCl}_{3}\right)$; IR (film) 3487, 2933, 2859, $1083 \mathrm{~cm}^{-1} ;{ }^{1} \mathrm{H}$ NMR $\left(600 \mathrm{MHz}, \mathrm{CDCl}_{3}\right) \delta 7.34-$ $7.25(10 \mathrm{H}, \mathrm{m}), 4.54$ and 4.35 (each 1H, d, $J=11.7 \mathrm{~Hz}), 4.52$ and 4.44 (each $1 \mathrm{H}, \mathrm{d}, J=12.1 \mathrm{~Hz}), 4.14(1 \mathrm{H}$, dd, $J=10.2,4.9 \mathrm{~Hz}), 3.87$ (1H, dd, $J=12.2,4.3 \mathrm{~Hz}), 3.84(1 \mathrm{H}, \mathrm{ddd}, J=11.2,9.5,4.6 \mathrm{~Hz}), 3.82(1 \mathrm{H}, \mathrm{m})$, $3.81(1 \mathrm{H}, \mathrm{t}, J=10.3 \mathrm{~Hz}), 3.63(1 \mathrm{H}, \mathrm{ddd}, J=9.5,4.8,3.9 \mathrm{~Hz}), 3.55(1 \mathrm{H}, \mathrm{ddd}, J=9.2,8.2,6.1 \mathrm{~Hz}), 3.52$ $(1 \mathrm{H}, \mathrm{ddd}, J=11.2,9.5,4.8 \mathrm{~Hz}), 3.52(1 \mathrm{H}, \mathrm{ddd}, J=9.2,6.6,4.8 \mathrm{~Hz}), 3.42(1 \mathrm{H}, \mathrm{dt}, J=8.8,5.9 \mathrm{~Hz}), 3.40$ $(1 \mathrm{H}, \mathrm{ddd}, J=5.9,5.1,2.8 \mathrm{~Hz}), 3.33-3.25(4 \mathrm{H}, \mathrm{m}), 3.16(1 \mathrm{H}, \mathrm{ddd}, J=11.6,9.0,4.1 \mathrm{~Hz}), 3.01(1 \mathrm{H}, \mathrm{ddd}, J$ $=11.7,9.3,4.3 \mathrm{~Hz}), 2.95(1 \mathrm{H}, \mathrm{td}, J=8.3,4.6 \mathrm{~Hz}), 2.59(1 \mathrm{H}, \mathrm{t}, J=1.3 \mathrm{~Hz}), 2.45(1 \mathrm{H}$, dddd, $J=16.0,7.6$, 6.0, $1.3 \mathrm{~Hz}), 2.32-2.28(2 \mathrm{H}, \mathrm{m}), 2.11(1 \mathrm{H}, \mathrm{dt}, J=11.7,4.3 \mathrm{~Hz}), 1.94(1 \mathrm{H}, \mathrm{dddd}, J=14.7,7.4,5.9,1.0$ Hz), $1.90-1.72(8 \mathrm{H}, \mathrm{m}), 1.66(1 \mathrm{H}, \mathrm{ddt}, J=14.5,12.5,1.5 \mathrm{~Hz}), 1.61(1 \mathrm{H}, \mathrm{m}), 1.59(1 \mathrm{H}, \mathrm{q}, J=11.9 \mathrm{~Hz})$, $1.46(1 \mathrm{H}, \mathrm{q}, J=11.2 \mathrm{~Hz}), 1.42(1 \mathrm{H}, \mathrm{q}, J=11.6 \mathrm{~Hz}), 1.16(3 \mathrm{H}, \mathrm{s}), 1.03(9 \mathrm{H}, \mathrm{s}), 0.98(9 \mathrm{H}, \mathrm{s}) ;{ }^{13} \mathrm{C}$ NMR $\left(150 \mathrm{MHz}, \mathrm{CDCl}_{3}\right) \delta 138.55,138.48,128.32,128.31,127.7,127.6,127.50,127.49,84.1,83.7,82.1,81.5$, 81.1, 79.7, 79.6, 78.6, 78.4, 77.72, 77.69, 74.7, 74.0, 72.9, 72.8, 70.7, 68.8, 67.1, 66.8, 39.1, 38.6, 35.8, $34.9,32.3,30.3,28.4,28.0,27.4,27.0,23.9,22.6,19.9,14.5$; HRDARTMS $m / z$ calcd for $\mathrm{C}_{49} \mathrm{H}_{73} \mathrm{O}_{11} \mathrm{Si}$ $\left(\mathrm{MH}^{+}\right)$865.4917, found 865.4918.

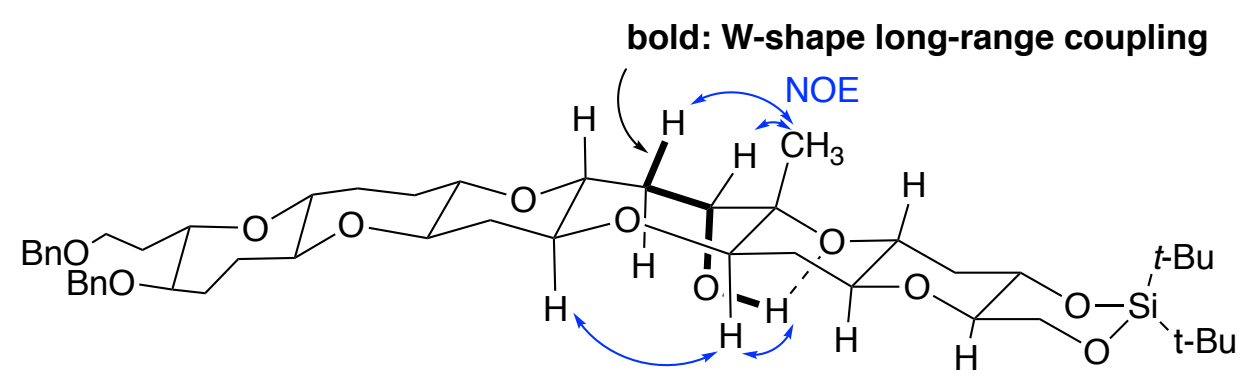

Figure S5. NOE and long-range coupling of 1 
Comparison of ${ }^{1} \mathrm{H}$ and ${ }^{13} \mathrm{C}$ spectra between the GHIJKL fragment 1 and gymnocin-B

Table S1. Comparison of chemical shifts of ${ }^{1} \mathrm{H}$ NMR spectra

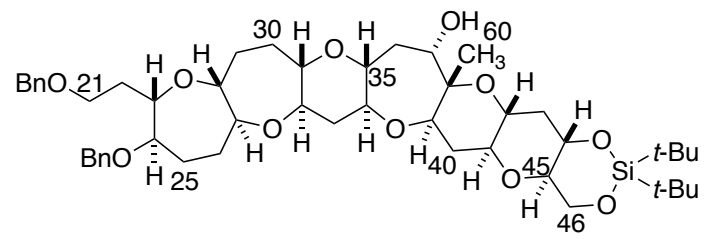

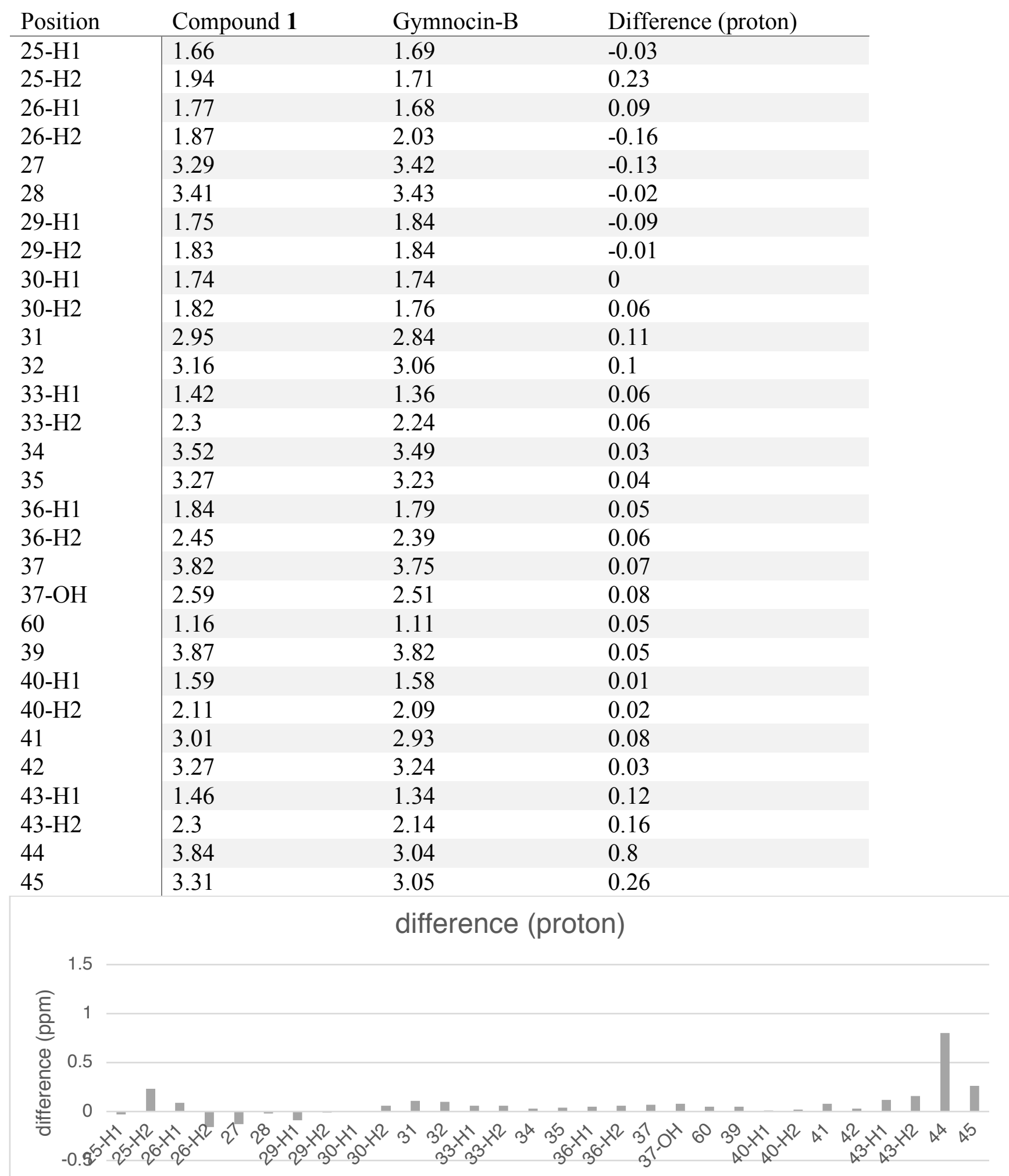


Table S2. Comparison of chemical shifts of ${ }^{13} \mathrm{C}$ NMR spectra

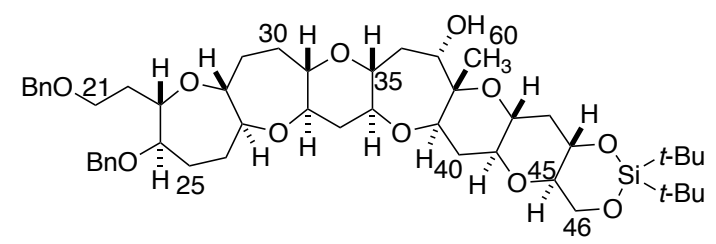

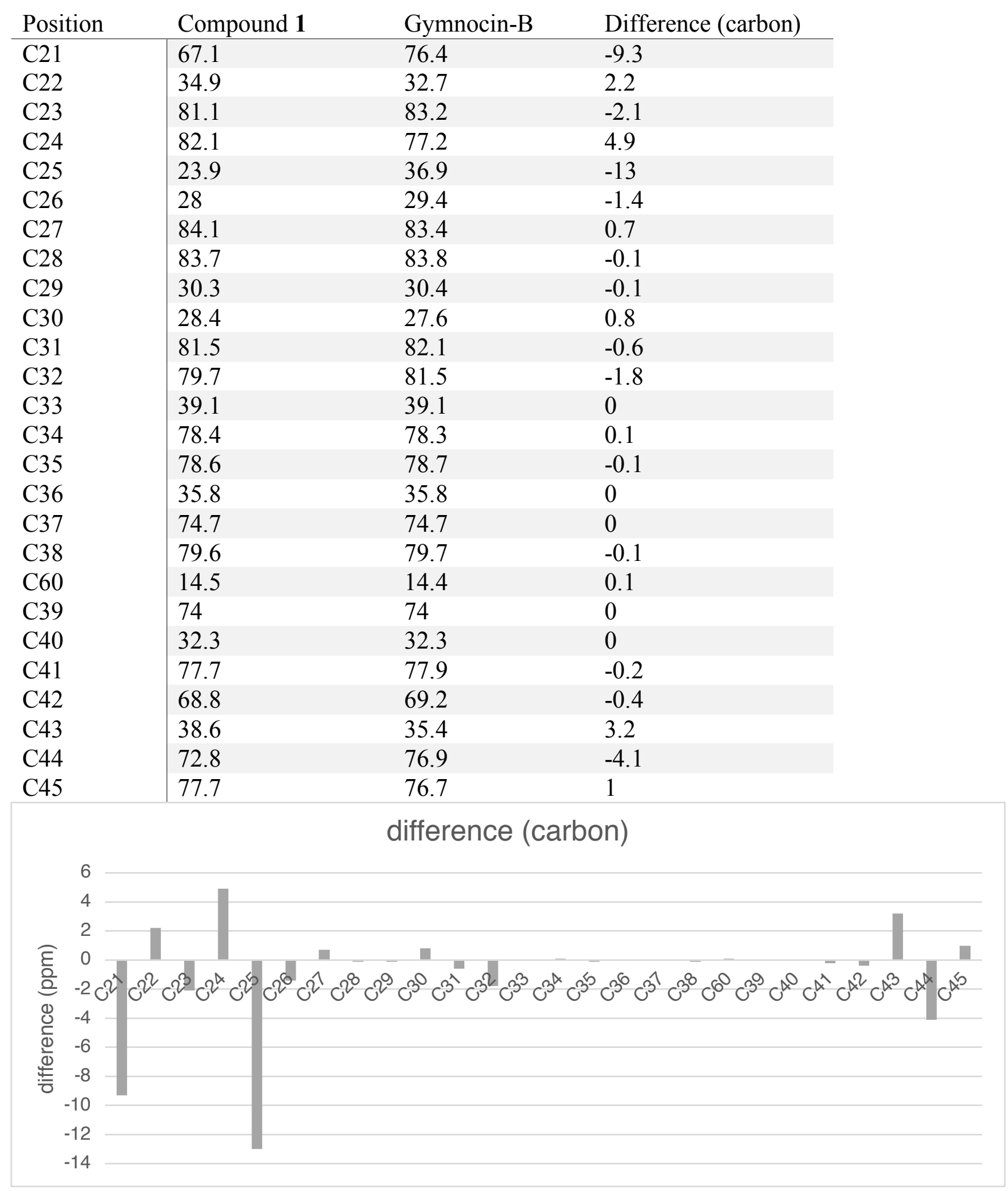




\section{Computational conformational analysis for bromoketones $6 a$ and $6 b$}

\section{Scheme S3}

\section{Equibrilium between $6 a$ and $6 b$}

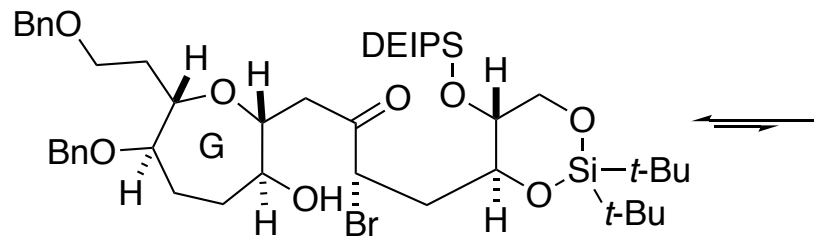

$6 a$

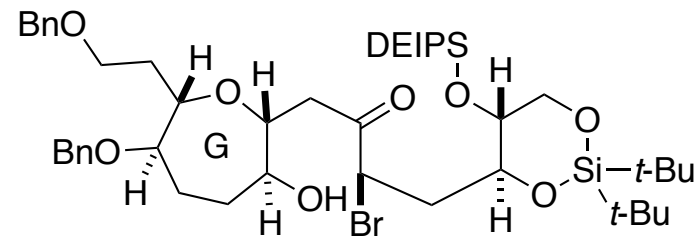

$6 b$

Model compounds S19a and S19b for the conformational analysis<smiles>CCC(=O)CC1OC2(CC)OC1(O)C(OC)CCC2O</smiles><smiles>CC[C@]1(C)O[C@H](CC(=O)C(C)(Br)Br)[C@@H](O)CC[C@H]1OC</smiles>

Computational conformational analysis for bromoketones $\mathbf{6 a}$ and $\mathbf{6 b}$ were performed for the model compounds S19a and S19b (Scheme S3). The calculation was carried out using Spartan'16 for Mac according to the following procedure.

1) Monte Carlo conformational search was performed with molecular mechanics calculation using the MMFF force field (619 conformations for S19a and 779 conformations for S19b).

2) Geometrical optimization at the $\mathrm{HF} / 3-21 \mathrm{G}$ level of theory for all of the conformations obtained by the MMFF search. All geometries within $+4 \mathrm{kcal} / \mathrm{mol}$ (42 conformations for S19a and 133 conformations for S19b, listed in Table S3 and Table S4) were further optimized at the M06-2X/6-31G(d) level of theory.

3) Vibrational analysis was carried out at $298.15 \mathrm{~K}$ for all geometries within $+3.3 \mathrm{kcal} / \mathrm{mol}$ at the M062X/6-31G(d) level of theory (15 conformations for $\mathbf{S 1 9 a}$ and 71 conformations for $\mathbf{S 1 9 b}$, no imaginary frequencies). 
a) The five most stable conformations for the major bromoketone model S19a.

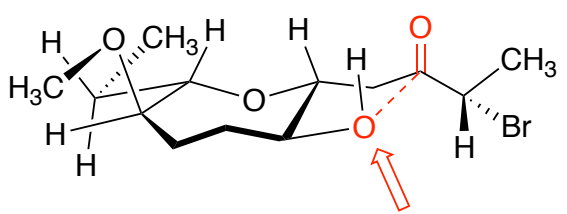

five-membered coordination

$0 \mathrm{kcal} / \mathrm{mol}$

S19a-M0006

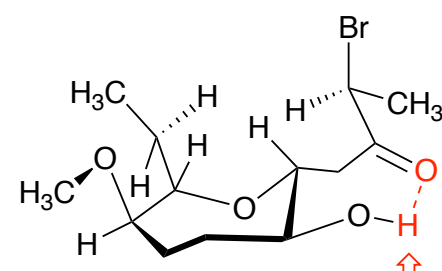

$+0.19 \mathrm{kcal} / \mathrm{mol}$

S19a-M0029

intramolecular hydrogen bond

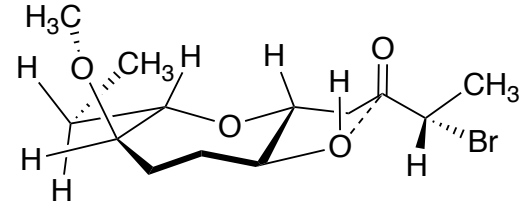

$+0.30 \mathrm{kcal} / \mathrm{mol}$ S19a-M0011

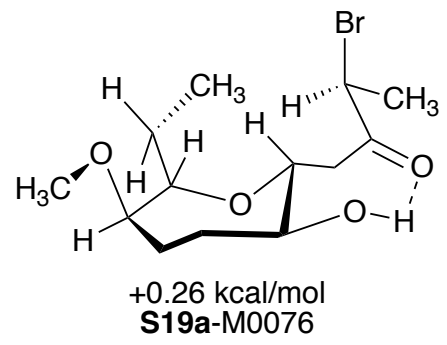

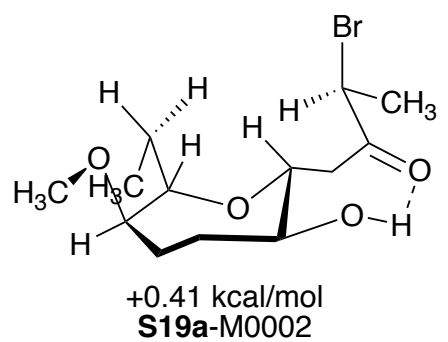

b) The five most stable conformations for the minor bromoketone model S19b
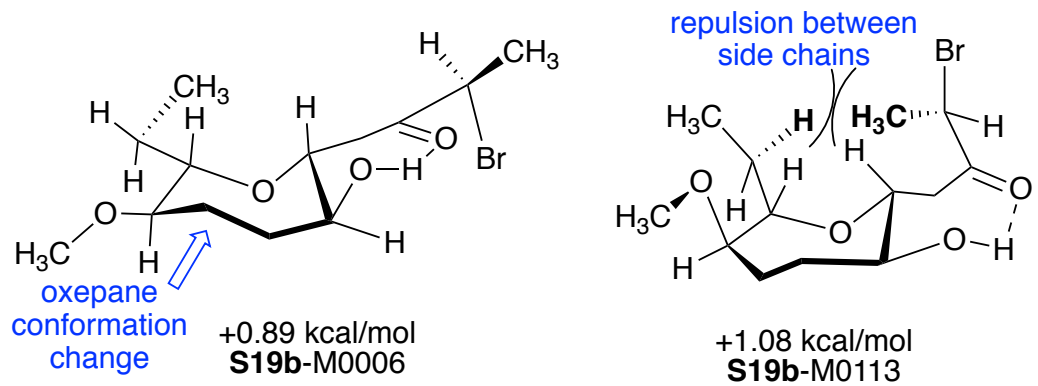

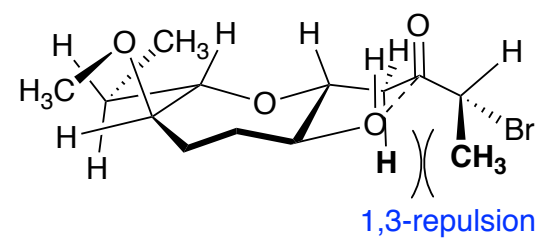

$+1.09 \mathrm{kcal} / \mathrm{mol}$

S19b-M0027
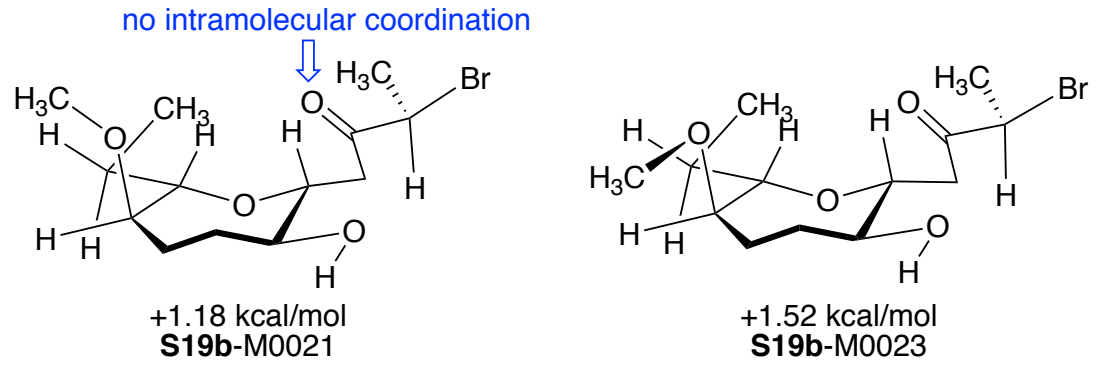

Figure S6. The five stable conformations for S19a and S19b and the calculated Gibbs energies relative to S19a0006 at M06-2X/6-31G(d) level of theory.

The calculated stable conformations for S19a and S19b are shown in Figure S6. The most stable conformation (S19a-M0006) for the major bromoketone model S19a involves a five-membered coordination between the hydroxy and the ketone groups. The similar conformation for the minor bromoketone model S19b (S19b-M0027) has the 1,3-repulsion between $\alpha$-methyl and $\alpha$-hydrogen, and the energy of S19b-M0027 is calculated to be higher by $1.09 \mathrm{kcal} / \mathrm{mol}$ than S19a-M0006. The second conformation of S19a has an intramolecular hydrogen bond to form a seven-membered coordination 
(S19a-M0029). The similar conformation for S19b (S19b-M0113) possesses a significant repulsion between side chains compared with S19a-M0029. Other conformations for S19b have also higher energy because of the strain oxepane conformation (S19b-M0006) or the absence of intramolecular coordination for the bromoketone side chain (S19b-M0021 and S19b-M0023).

Table S3. List of conformations for the major bromoketone model S19a

\begin{tabular}{|c|c|c|c|c|}
\hline Label & $\begin{array}{l}\text { Relative Total Energy } \\
\text { (kcal/mol, } \mathrm{HF} / 3-21 \mathrm{G} \text {, } \\
\text { relative to M0076 of } \\
\mathbf{S 1 9 a )}\end{array}$ & $\begin{array}{l}\text { Relative Total Energy } \\
(\mathrm{kcal} / \mathrm{mol} \text {, M06-2X/6- } \\
31 \mathrm{G}(\mathrm{d}) \text {, relative to } \\
\mathrm{M} 0076 \text { of } \mathbf{S 1 9 a})\end{array}$ & $\begin{array}{l}\text { Relative Gibbs Energy } \\
(\mathrm{kcal} / \mathrm{mol} \text {, M06-2X/6- } \\
31 \mathrm{G}(\mathrm{d}) \text {, relative to } \\
\text { M0006 of } \mathbf{S 1 9 a )}\end{array}$ & RANK \\
\hline M0076 & 0 & 0 & 0.259454 & 3 \\
\hline M0029 & 0.22 & 0.64 & 0.188255 & 2 \\
\hline M0003 & 1.14 & 1.18 & 0.985233 & 8 \\
\hline M0002 & 1.15 & 0.33 & 0.40988 & 5 \\
\hline M0102 & 1.38 & 1.38 & 0.907841 & 6 \\
\hline M0006 & 1.77 & 1.16 & 0 & 1 \\
\hline M0011 & 2.00 & 1.83 & 0.300883 & 4 \\
\hline M0005 & 2.46 & 1.58 & 1.210237 & 10 \\
\hline M0032 & 2.60 & 3.78 & - & \\
\hline M0018 & 2.90 & 2.02 & 0.984926 & 7 \\
\hline M0017 & 3.06 & 4.01 & - & \\
\hline M0019 & 3.17 & 2.17 & 1.318884 & 11 \\
\hline M0559 & 3.29 & 4.09 & - & \\
\hline M0025 & 3.34 & 3.19 & 1.609664 & 12 \\
\hline M0010 & 3.37 & 3.44 & - & \\
\hline M0028 & 3.38 & 4.17 & - & \\
\hline M0117 & 3.55 & 3.80 & - & \\
\hline M0014 & 3.61 & 3.99 & - & \\
\hline M0015 & 3.64 & 1.79 & 1.071956 & 9 \\
\hline M0009 & 3.68 & 2.81 & 2.144205 & 13 \\
\hline M0057 & 3.86 & 3.74 & - & \\
\hline M0064 & 3.88 & 3.89 & - & \\
\hline M0039 & 4.00 & 4.77 & - & \\
\hline
\end{tabular}

Table S4. List of conformations for the minor bromoketone model S19b

\begin{tabular}{|c|c|c|c|c|}
\hline Label & $\begin{array}{l}\text { Relative Total Energy } \\
\text { (kcal/mol, HF/3-21G, } \\
\text { relative to M0036 of } \\
\text { S19b) }\end{array}$ & $\begin{array}{l}\text { Relative Total Energy } \\
\text { (kcal/mol, M06-2X/6- } \\
31 \mathrm{G}(\mathrm{d}) \text {, relative to } \\
\mathrm{M} 0014 \text { of } \mathbf{S 1 9 b})\end{array}$ & $\begin{array}{l}\text { Relative Gibbs Energy } \\
(\mathrm{kcal} / \mathrm{mol} \text {, M06-2X/6- } \\
31 \mathrm{G}(\mathrm{d}) \text {, relative to } \\
\mathrm{M} 0006 \text { of } \mathbf{S 1 9 a})\end{array}$ & RANK \\
\hline M0036 & 0 & 2.40 & 2.569005 & 27 \\
\hline M0078 & 0.37 & 3.08 & 3.906736 & 68 \\
\hline M0006 & 0.44 & 2.70 & 0.879849 & 1 \\
\hline M0001 & 0.54 & 1.22 & (M0010) & \\
\hline M0010 & 0.56 & 1.21 & 1.982093 & 13 \\
\hline
\end{tabular}




\begin{tabular}{|c|c|c|c|c|}
\hline M0018 & 0.59 & 2.62 & 3.144583 & 48 \\
\hline M0113 & 0.61 & 0.84 & 1.091965 & 3 \\
\hline M0005 & 0.84 & 2.09 & 2.726985 & 31 \\
\hline M0075 & 0.84 & 3.03 & 3.492321 & 64 \\
\hline M0028 & 1.01 & 1.54 & 2.255305 & 15 \\
\hline M0027 & 1.04 & 0.39 & 1.084241 & 2 \\
\hline M0076 & 1.09 & 2.00 & 2.465267 & 25 \\
\hline M0025 & 1.26 & 3.02 & 2.592338 & 28 \\
\hline M0041 & 1.29 & 1.83 & 2.392394 & 21 \\
\hline M0009 & 1.38 & 2.09 & 1.60746 & 7 \\
\hline M0029 & 1.38 & 3.29 & 2.366187 & 20 \\
\hline M0236 & 1.46 & 2.42 & 2.964264 & 39 \\
\hline M0095 & 1.60 & 3.26 & 4.296816 & 71 \\
\hline M0002 & 1.62 & 1.65 & 2.783091 & 34 \\
\hline M0417 & 1.62 & 3.87 & - & \\
\hline M0021 & 1.63 & 2.35 & 1.181268 & 4 \\
\hline M0003 & 1.69 & 2.41 & 3.174838 & 51 \\
\hline M0476 & 1.73 & 1.77 & 1.533184 & 6 \\
\hline M0026 & 1.73 & 3.21 & 3.380126 & 61 \\
\hline M0342 & 1.74 & 0.45 & (M0013) & \\
\hline M0013 & 1.74 & 0.45 & 2.429907 & 22 \\
\hline M0071 & 1.75 & 1.69 & 1.841128 & 12 \\
\hline M0099 & 1.77 & 2.87 & 3.250821 & 57 \\
\hline M0023 & 1.83 & 1.98 & 1.52807 & 5 \\
\hline M0273 & 1.84 & 4.08 & - & \\
\hline M0014 & 1.88 & 0 & 1.830446 & 11 \\
\hline M0024 & 1.97 & 3.04 & 2.552588 & 26 \\
\hline M0037 & 1.97 & 3.11 & 3.12091 & 46 \\
\hline M0031 & 2.09 & 4.27 & - & \\
\hline M0022 & 2.12 & 2.86 & 3.110286 & 44 \\
\hline M0109 & 2.18 & 4.08 & - & \\
\hline M0082 & 2.19 & 1.98 & 2.438576 & 14 \\
\hline M0012 & 2.19 & 2.40 & 2.126178 & 23 \\
\hline M0040 & 2.25 & 2.93 & 2.592727 & 29 \\
\hline M0061 & 2.26 & 1.13 & 2.766524 & 33 \\
\hline M0120 & 2.30 & 4.52 & - & \\
\hline M0066 & 2.32 & 3.47 & - & \\
\hline M0348 & 2.36 & 3.48 & - & \\
\hline M0102 & 2.39 & 2.64 & 3.229628 & 56 \\
\hline M0042 & 2.42 & 3.62 & - & \\
\hline M0218 & 2.42 & 4.41 & - & \\
\hline M0199 & 2.50 & 5.14 & - & \\
\hline M0062 & 2.51 & 1.49 & 2.341235 & 19 \\
\hline M0403 & 2.52 & 4.63 & - & \\
\hline M0115 & 2.58 & 3.29 & 3.383534 & 62 \\
\hline M0054 & 2.61 & 2.45 & 2.444164 & 24 \\
\hline M0193 & 2.62 & 3.26 & 3.514486 & 65 \\
\hline M0211 & 2.67 & 4.14 & - & \\
\hline
\end{tabular}




\begin{tabular}{|c|c|c|c|c|}
\hline M0131 & 2.70 & 3.56 & - & \\
\hline M0050 & 2.71 & 2.67 & 2.26754 & 16 \\
\hline M0104 & 2.71 & 5.02 & - & \\
\hline M0046 & 2.73 & 2.59 & 1.752638 & 9 \\
\hline M0064 & 2.77 & 3.16 & 2.760633 & 32 \\
\hline M0190 & 2.79 & 4.74 & - & \\
\hline M0192 & 2.79 & 5.22 & - & \\
\hline M0039 & 2.81 & 2.91 & 2.834668 & 35 \\
\hline M0074 & 2.81 & 4.10 & - & \\
\hline M0340 & 2.84 & 4.43 & - & \\
\hline M0413 & 2.86 & 2.91 & 3.99225 & 55 \\
\hline M0516 & 2.87 & 3.43 & - & \\
\hline M0051 & 2.91 & 1.67 & 3.15155 & 18 \\
\hline M0640 & 2.91 & 2.95 & 2.309192 & 49 \\
\hline M0157 & 2.93 & 3.32 & - & 60 \\
\hline M0111 & 3.00 & 2.45 & 2.277514 & 17 \\
\hline M0053 & 3.04 & 3.18 & 3.114737 & 45 \\
\hline M0034 & 3.05 & 2.66 & 3.222977 & 54 \\
\hline M0015 & 3.05 & 2.95 & 4.016307 & 70 \\
\hline M0144 & 3.06 & 4.25 & - & \\
\hline M0129 & 3.09 & 3.29 & 3.171179 & 50 \\
\hline M0242 & 3.11 & 4.55 & - & \\
\hline M0045 & 3.12 & 3.69 & - & \\
\hline M0251 & 3.12 & 4.09 & - & \\
\hline M0165 & 3.12 & 4.83 & - & \\
\hline M0136 & 3.14 & 3.41 & - & \\
\hline M0070 & 3.17 & 3.46 & - & \\
\hline M0124 & 3.17 & 4.95 & - & \\
\hline M0083 & 3.19 & 2.20 & 3.108141 & 43 \\
\hline M0100 & 3.22 & 2.45 & 2.87224 & 36 \\
\hline M0346 & 3.22 & 4.99 & - & \\
\hline M0176 & 3.22 & 5.74 & - & \\
\hline M0073 & 3.24 & 2.94 & 1.788836 & 10 \\
\hline M0489 & 3.25 & 1.19 & 3.297844 & 59 \\
\hline M0268 & 3.32 & 4.35 & - & \\
\hline M0065 & 3.34 & 3.20 & 4.014726 & 69 \\
\hline M0156 & 3.34 & 5.38 & - & \\
\hline M0107 & 3.35 & 3.06 & 3.0655 & 41 \\
\hline M0195 & 3.35 & 3.14 & 3.071737 & 42 \\
\hline M0147 & 3.35 & 3.63 & - & \\
\hline M0097 & 3.36 & 4.33 & - & \\
\hline M0166 & 3.37 & 3.30 & 3.272387 & 58 \\
\hline M0154 & 3.38 & 4.91 & - & \\
\hline M0399 & 3.41 & 2.87 & 3.773577 & 40 \\
\hline M0206 & 3.41 & 2.89 & 3.044924 & 67 \\
\hline M0532 & 3.44 & 3.04 & 2.67215 & 30 \\
\hline M0081 & 3.44 & 3.27 & 3.181706 & 52 \\
\hline M0277 & 3.45 & 4.99 & - & \\
\hline
\end{tabular}




\begin{tabular}{|c|c|c|c|c|}
\hline M0092 & 3.46 & 2.66 & 3.387158 & 63 \\
\hline M0294 & 3.48 & 5.73 & - & \\
\hline M0524 & 3.49 & 3.09 & 2.923657 & 38 \\
\hline M0465 & 3.49 & 3.45 & - & \\
\hline M0160 & 3.53 & 5.64 & - & \\
\hline M0233 & 3.54 & 2.71 & 1.635019 & 8 \\
\hline M0132 & 3.54 & 3.03 & 3.126584 & 47 \\
\hline M0088 & 3.55 & 4.60 & - & \\
\hline M0090 & 3.59 & 3.33 & - & \\
\hline M0241 & 3.60 & 5.61 & - & \\
\hline M0118 & 3.67 & 4.26 & - & \\
\hline M0108 & 3.67 & 4.38 & - & \\
\hline M0110 & 3.69 & 3.67 & - & \\
\hline M0397 & 3.75 & 4.80 & - & \\
\hline M0094 & 3.76 & 3.15 & 2.916677 & \\
\hline M0063 & 3.76 & 3.81 & - & 37 \\
\hline M0283 & 3.78 & 3.73 & - & \\
\hline M0130 & 3.81 & 4.63 & - & \\
\hline M0016 & 3.81 & 5.39 & - & \\
\hline M0245 & 3.81 & 5.56 & - & \\
\hline M0574 & 3.84 & 4.06 & - & \\
\hline M0216 & 3.84 & 4.08 & - & \\
\hline M0043 & 3.88 & 3.15 & 3.530587 & \\
\hline M0371 & 3.88 & 4.96 & - & 66 \\
\hline M0173 & 3.89 & 2.64 & (M0102) & \\
\hline M0204 & 3.91 & 2.64 & 3.183872 & \\
\hline M0223 & 3.93 & 5.85 & - & 53 \\
\hline M0056 & 3.94 & 3.46 & - & \\
\hline M0163 & 3.95 & 3.57 & - & \\
\hline M0209 & 3.97 & 3.61 & - & \\
\hline M0119 & 3.98 & 6.28 & - & \\
\hline
\end{tabular}


Cartesian coordination for the calculated stable conformations

S19a-M0006

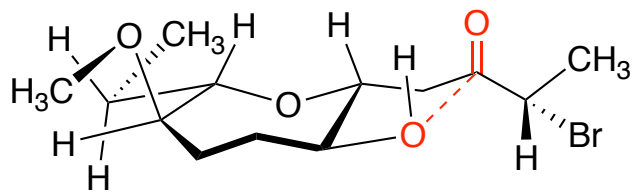

$\begin{array}{lrrr}\mathrm{C} & 1.1584 & 2.1088 & -0.6165 \\ \mathrm{H} & 1.6600 & 3.0911 & -0.5935 \\ \mathrm{C} & 0.3169 & 1.9835 & 0.6678 \\ \mathrm{H} & -0.6967 & 2.3363 & 0.4205 \\ \mathrm{O} & 0.2478 & 0.6567 & 1.1570 \\ \mathrm{C} & -0.3180 & -0.3023 & 0.2826 \\ \mathrm{H} & -0.8904 & 0.1887 & -0.5177 \\ \mathrm{C} & 0.8316 & -1.1219 & -0.3146 \\ \mathrm{C} & 1.7054 & -0.3198 & -1.2747 \\ \mathrm{C} & 0.8909 & 2.8235 & 1.8028 \\ \mathrm{H} & 0.9246 & 3.8678 & 1.4715 \\ \mathrm{H} & 1.9282 & 2.5098 & 1.9805 \\ \mathrm{O} & 0.2582 & 2.1020 & -1.7126 \\ \mathrm{C} & -1.2638 & -1.1838 & 1.0990 \\ \mathrm{H} & -1.9964 & -0.5375 & 1.5937 \\ \mathrm{O} & 0.3506 & -2.3024 & -0.9474 \\ \mathrm{H} & 2.5467 & -0.9566 & -1.5669 \\ \mathrm{H} & 1.1179 & -0.1292 & -2.1830 \\ \mathrm{C} & 2.2344 & 1.0163 & -0.7397 \\ \mathrm{H} & 2.7040 & 0.8775 & 0.2412 \\ \mathrm{H} & 3.0219 & 1.3692 & -1.4146 \\ \mathrm{H} & 1.4366 & -1.4731 & 0.5302 \\ \mathrm{H} & -0.7058 & -1.7141 & 1.8779 \\ \mathrm{H} & -0.1868 & -2.0269 & -1.7079 \\ \mathrm{C} & 0.8462 & 2.5078 & -2.9245 \\ \mathrm{H} & 1.5873 & 1.7845 & -3.2916 \\ \mathrm{H} & 1.3387 & 3.4849 & -2.8148 \\ \mathrm{H} & 0.0432 & 2.5922 & -3.6587 \\ \mathrm{C} & -2.0069 & -2.1461 & 0.1941 \\ \mathrm{O} & -2.6131 & -1.7605 & -0.7813 \\ \mathrm{H} & -1.9505 & -3.6305 & 0.5348 \\ \mathrm{H} & -0.9364 & -3.8940 & 0.8364 \\ \mathrm{Br} & -3.0253 & -3.8408 & 2.1812 \\ \mathrm{C} & -2.4736 & -4.5143 & -0.5715 \\ \mathrm{H} & -2.4758 & -5.5619 & -0.2662 \\ \mathrm{H} & -3.4815 & -4.2122 & -0.8603 \\ \mathrm{H} & -1.8168 & -4.3984 & -1.4401 \\ 0.0794 & 2.7040 & 3.0887 \\ \mathrm{H} & 0.4973 & 3.3373 & 3.8759 \\ \mathrm{H} & -0694 & 1.6707 & 3.4412 \\ \mathrm{H} & & 3.0136 & 2.9210\end{array}$




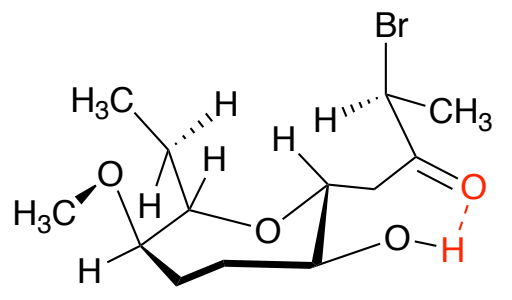

$\begin{array}{lrrr}\mathrm{C} & 0.7251 & 0.3164 & 1.9636 \\ \mathrm{H} & 0.9055 & -0.2050 & 2.9186 \\ \mathrm{C} & -0.5139 & -0.3285 & 1.3147 \\ \mathrm{H} & -1.3874 & 0.2382 & 1.6739 \\ \mathrm{O} & -0.4849 & -0.2973 & -0.1036 \\ \mathrm{C} & -0.2874 & 0.9804 & -0.6978 \\ \mathrm{H} & -0.4807 & 1.7777 & 0.0307 \\ \mathrm{C} & 1.1726 & 1.0510 & -1.1727 \\ \mathrm{C} & 2.1282 & 1.2438 & -0.0052 \\ \mathrm{C} & -0.6644 & -1.7997 & 1.6920 \\ \mathrm{H} & 0.2910 & -2.3121 & 1.5193 \\ \mathrm{H} & -1.3849 & -2.2390 & 0.9942 \\ \mathrm{O} & 0.3920 & 1.6626 & 2.2596 \\ \mathrm{C} & -1.2971 & 1.1336 & -1.8475 \\ \mathrm{H} & -1.1930 & 2.1338 & -2.2741 \\ \mathrm{O} & 1.3738 & 2.1164 & -2.0759 \\ \mathrm{H} & 3.1470 & 1.2249 & -0.4047 \\ \mathrm{H} & 1.9623 & 2.2492 & 0.3991 \\ \mathrm{C} & 2.0004 & 0.1997 & 1.1095 \\ \mathrm{H} & 2.0424 & -0.8124 & 0.6875 \\ \mathrm{H} & 2.8696 & 0.2912 & 1.7701 \\ \mathrm{H} & 1.3912 & 0.0844 & -1.6536 \\ \mathrm{H} & -2.3072 & 1.0116 & -1.4486 \\ \mathrm{H} & 1.0373 & 1.8151 & -2.9372 \\ \mathrm{C} & 1.3128 & 2.2967 & 3.1139 \\ \mathrm{H} & 2.2899 & 2.4458 & 2.6357 \\ \mathrm{H} & 1.4592 & 1.7146 & 4.0355 \\ \mathrm{H} & 0.8938 & 3.2714 & 3.3694 \\ \mathrm{C} & -1.0061 & 0.1149 & -2.9238 \\ \mathrm{O} & -0.1442 & 0.2995 & -3.7601 \\ \mathrm{H} & -1.7567 & -1.2095 & -2.8749 \\ \mathrm{H} & -1.8550 & -1.5125 & -1.8307 \\ \mathrm{Br} & -3.6005 & -0.8052 & -3.4533 \\ \mathrm{C} & -1.1436 & -2.2777 & -3.7502 \\ \mathrm{H} & -1.0678 & -1.9309 & -4.7821 \\ \mathrm{H} & -0.1332 & -2.4919 & -3.3873 \\ \mathrm{H} & -1.7359 & -3.1936 & -3.7155 \\ -1.1416 & -2.0190 & 3.1252 \\ \mathrm{H} & -1.2576 & -3.0858 & 3.3338 \\ \mathrm{H} & -1106 & -1.5375 & 3.2907 \\ \mathrm{H} & -1.6151 & 3.8618\end{array}$




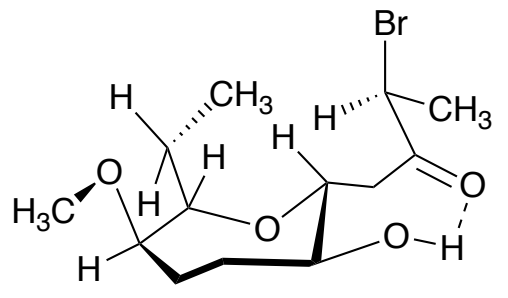

$\begin{array}{lrrr}\mathrm{C} & -1.9995 & -0.3039 & -1.1464 \\ \mathrm{H} & -3.0052 & 0.1368 & -1.2610 \\ \mathrm{C} & -1.5497 & -0.0233 & 0.3001 \\ \mathrm{H} & -1.9066 & -0.8667 & 0.9122 \\ \mathrm{O} & -0.1442 & 0.0948 & 0.4470 \\ \mathrm{C} & 0.6291 & -0.9762 & -0.0844 \\ \mathrm{H} & -0.0024 & -1.8549 & -0.2620 \\ \mathrm{C} & 1.2302 & -0.4851 & -1.4124 \\ \mathrm{C} & 0.1887 & -0.4865 & -2.5215 \\ \mathrm{C} & -2.1555 & 1.2775 & 0.8183 \\ \mathrm{H} & -3.2440 & 1.1526 & 0.8396 \\ \mathrm{H} & -1.9417 & 2.0809 & 0.1013 \\ \mathrm{O} & -2.1224 & -1.7093 & -1.2885 \\ \mathrm{C} & 1.6963 & -1.3634 & 0.9505 \\ \mathrm{H} & 2.2855 & -2.1913 & 0.5496 \\ \mathrm{O} & 2.3108 & -1.2919 & -1.8284 \\ \mathrm{H} & 0.6623 & -0.0823 & -3.4216 \\ \mathrm{H} & -0.0693 & -1.5300 & -2.7371 \\ \mathrm{C} & -1.0794 & 0.3175 & -2.2125 \\ \mathrm{H} & -0.8130 & 1.3317 & -1.8895 \\ \mathrm{H} & -1.6505 & 0.4318 & -3.1403 \\ \mathrm{H} & 1.5624 & 0.5496 & -1.2377 \\ \mathrm{H} & 1.2013 & -1.6829 & 1.8714 \\ \mathrm{H} & 3.0803 & -1.0000 & -1.3104 \\ \mathrm{C} & -2.7962 & -2.0991 & -2.4608 \\ \mathrm{H} & -2.9459 & -3.1782 & -2.4000 \\ \mathrm{H} & -2.2192 & -1.8719 & -3.3668 \\ \mathrm{H} & -3.7749 & -1.6029 & -2.5351 \\ \mathrm{C} & 2.6054 & -0.1860 & 1.2037 \\ \mathrm{O} & 3.4990 & 0.1126 & 0.4368 \\ \mathrm{C} & 2.3033 & 0.6821 & 2.4168 \\ \mathrm{H} & 1.2235 & 0.7039 & 2.5716 \\ \mathrm{Br} & 3.0001 & -0.3248 & 3.9673 \\ \mathrm{C} & 2.9218 & 2.0577 & 2.3419 \\ \mathrm{H} & 2.7162 & 2.6259 & 3.2508 \\ \mathrm{H} & 4.0000 & 1.9851 & 2.1908 \\ \mathrm{H} & 2.4909 & 2.5867 & 1.4850 \\ \mathrm{H} & -1.6452 & 1.6705 & 2.2009 \\ -1.7485 & 0.8406 & 2.9086 \\ -2.2042 & 2.5231 & 2.5959 \\ \mathrm{H} & & & \\ \mathrm{H} & & & \\ \mathrm{H} & -5898 & 1.9495 & 2.1562\end{array}$




\section{S19a-M0011}

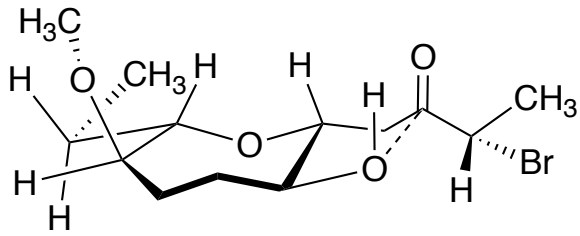

$\begin{array}{lrrr}\mathrm{C} & 1.4423 & 1.1181 & -1.6975 \\ \mathrm{H} & 2.1208 & 1.8372 & -2.1857 \\ \mathrm{C} & 1.0052 & 1.7331 & -0.3488 \\ \mathrm{H} & 0.0628 & 2.2828 & -0.5138 \\ \mathrm{O} & 0.7912 & 0.7584 & 0.6549 \\ \mathrm{C} & -0.1886 & -0.2234 & 0.3689 \\ \mathrm{H} & -0.8335 & 0.0995 & -0.4606 \\ \mathrm{C} & 0.5469 & -1.5155 & -0.0048 \\ \mathrm{C} & 1.2659 & -1.4288 & -1.3468 \\ \mathrm{C} & 2.0541 & 2.6926 & 0.2013 \\ \mathrm{H} & 2.2112 & 3.4858 & -0.5395 \\ \mathrm{H} & 3.0051 & 2.1521 & 0.2966 \\ \mathrm{O} & 0.3371 & 0.8922 & -2.5583 \\ \mathrm{C} & -1.0483 & -0.4176 & 1.6179 \\ \mathrm{H} & -1.4456 & 0.5572 & 1.9203 \\ \mathrm{O} & -0.3318 & -2.6350 & 0.0197 \\ \mathrm{H} & 1.8505 & -2.3463 & -1.4701 \\ \mathrm{H} & 0.5126 & -1.4168 & -2.1443 \\ \mathrm{C} & 2.1829 & -0.2152 & -1.5396 \\ \mathrm{H} & 2.8909 & -0.1312 & -0.7066 \\ \mathrm{H} & 2.7681 & -0.3799 & -2.4495 \\ \mathrm{H} & 1.2729 & -1.7017 & 0.7962 \\ \mathrm{H} & -0.4374 & -0.7986 & 2.4430 \\ \mathrm{H} & -0.9544 & -2.5321 & -0.7185 \\ \mathrm{C} & -0.0951 & 2.0439 & -3.2402 \\ \mathrm{H} & 0.7031 & 2.4445 & -3.8815 \\ \mathrm{H} & -0.4210 & 2.8383 & -2.5551 \\ \mathrm{H} & -0.9423 & 1.7505 & -3.8621 \\ \mathrm{C} & -2.2154 & -1.3367 & 1.3174 \\ \mathrm{O} & -2.9505 & -1.1399 & 0.3749 \\ \mathrm{C} & -2.4290 & -2.5398 & 2.2287 \\ \mathrm{H} & -1.4682 & -3.0183 & 2.4193 \\ \mathrm{Br} & -2.9725 & -1.8036 & 3.9804 \\ \mathrm{C} & -3.4635 & -3.5067 & 1.7045 \\ \mathrm{H} & -3.6249 & -4.3237 & 2.4097 \\ \mathrm{H} & -4.4076 & -2.9940 & 1.5135 \\ \mathrm{H} & -3.0994 & -3.9201 & 0.7582 \\ & 1.6557 & 3.2948 & 1.5446 \\ \mathrm{H} & 2.4177 & 3.9913 & 1.9044 \\ 1.5208 & 2.5095 & 2.2909 \\ 0.7112 & 3.8430 & 1.4580\end{array}$




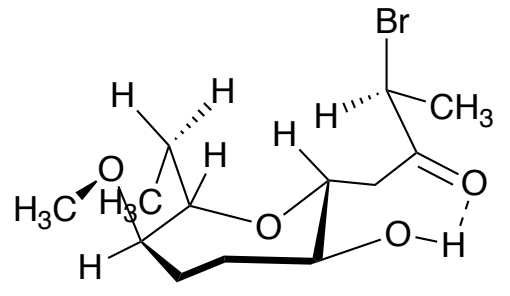

$\begin{array}{lrrr}\mathrm{C} & 0.8307 & 1.8532 & 0.9410 \\ \mathrm{H} & 1.6116 & 2.1442 & 1.6657 \\ \mathrm{C} & 0.1064 & 0.6141 & 1.5101 \\ \mathrm{H} & -0.7883 & 0.9993 & 2.0210 \\ \mathrm{O} & -0.2945 & -0.3135 & 0.5150 \\ \mathrm{C} & -1.1621 & 0.2003 & -0.4880 \\ \mathrm{H} & -1.6043 & 1.1501 & -0.1590 \\ \mathrm{C} & -0.3302 & 0.4306 & -1.7597 \\ \mathrm{C} & 0.5555 & 1.6601 & -1.6279 \\ \mathrm{C} & 0.9471 & -0.1714 & 2.5132 \\ \mathrm{H} & 0.3230 & -0.9896 & 2.8895 \\ \mathrm{H} & 1.1642 & 0.4816 & 3.3663 \\ \mathrm{O} & -0.1333 & 2.8939 & 0.8977 \\ \mathrm{C} & -2.3028 & -0.8096 & -0.6938 \\ \mathrm{H} & -3.0109 & -0.3987 & -1.4170 \\ \mathrm{O} & -1.1542 & 0.6073 & -2.8912 \\ \mathrm{H} & 1.1400 & 1.7442 & -2.5495 \\ \mathrm{H} & -0.0961 & 2.5403 & -1.5798 \\ \mathrm{C} & 1.5046 & 1.6339 & -0.4250 \\ \mathrm{H} & 2.0489 & 0.6834 & -0.3999 \\ \mathrm{H} & 2.2600 & 2.4159 & -0.5625 \\ \mathrm{H} & 0.3128 & -0.4561 & -1.8791 \\ \mathrm{H} & -2.8085 & -0.9702 & 0.2618 \\ \mathrm{H} & -1.4455 & -0.2803 & -3.1617 \\ \mathrm{C} & 0.4156 & 4.1652 & 0.6482 \\ \mathrm{H} & 0.8026 & 4.2581 & -0.3751 \\ \mathrm{H} & 1.2310 & 4.3867 & 1.3524 \\ \mathrm{H} & -0.3855 & 4.8928 & 0.7885 \\ \mathrm{C} & -1.7424 & -2.1007 & -1.2412 \\ \mathrm{H} & -1.5244 & -2.2476 & -2.4275 \\ \mathrm{C} & -1.3507 & -3.1858 & -0.2467 \\ \mathrm{H} & -0.9248 & -2.7070 & 0.6373 \\ \mathrm{Br} & -3.0449 & -3.9791 & 0.3821 \\ \mathrm{C} & -0.4521 & -4.2453 & -0.8409 \\ \mathrm{H} & -0.2334 & -5.0229 & -0.1071 \\ \mathrm{H} & -0.9174 & -4.6933 & -1.7206 \\ \mathrm{H} & 0.4863 & -3.7753 & -1.1533 \\ \mathrm{H} & 2.2426 & -0.7407 & 1.9382 \\ \mathrm{H} & 2.9444 & 0.0521 & 1.6584 \\ \mathrm{H} & & -1.3426 & 1.0482 \\ \mathrm{H} & -1.3776 & 2.6723\end{array}$




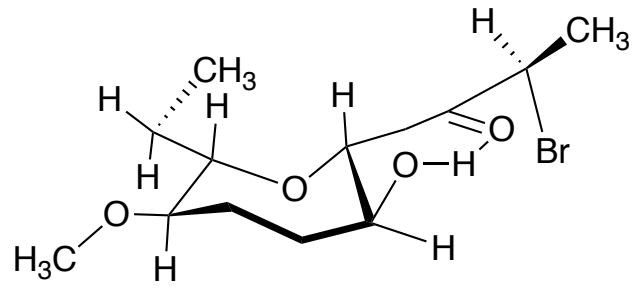

$\begin{array}{lrrr}\mathrm{C} & -0.2772 & 0.6650 & -2.5256 \\ \mathrm{H} & -1.3434 & 0.5390 & -2.7880 \\ \mathrm{C} & 0.1522 & -0.5598 & -1.7154 \\ \mathrm{H} & 1.2399 & -0.4854 & -1.5475 \\ \mathrm{O} & -0.5200 & -0.5978 & -0.4644 \\ \mathrm{C} & 0.0986 & 0.0775 & 0.6122 \\ \mathrm{H} & 1.1905 & 0.0657 & 0.4778 \\ \mathrm{C} & -0.3195 & 1.5701 & 0.7409 \\ \mathrm{C} & -0.9783 & 2.1103 & -0.5216 \\ \mathrm{C} & -0.1583 & -1.8647 & -2.4355 \\ \mathrm{H} & 0.2820 & -1.8103 & -3.4351 \\ \mathrm{H} & -1.2467 & -1.9334 & -2.5586 \\ \mathrm{O} & 0.5094 & 0.6355 & -3.7032 \\ \mathrm{C} & -0.2754 & -0.7390 & 1.8466 \\ \mathrm{H} & 0.0439 & -1.7812 & 1.7061 \\ \mathrm{O} & 0.8078 & 2.3826 & 0.9942 \\ \mathrm{H} & -1.9381 & 1.6045 & -0.6747 \\ \mathrm{H} & -1.1906 & 3.1658 & -0.3261 \\ \mathrm{C} & -0.0989 & 1.9830 & -1.7722 \\ \mathrm{H} & -0.3359 & 2.8006 & -2.4612 \\ \mathrm{H} & 0.9558 & 2.1028 & -1.4971 \\ \mathrm{H} & -1.0437 & 1.6622 & 1.5694 \\ \mathrm{H} & -1.3672 & -0.7700 & 1.9512 \\ \mathrm{H} & 1.2323 & 2.0327 & 1.7948 \\ \mathrm{C} & -0.0512 & 1.3239 & -4.7926 \\ \mathrm{H} & -0.1539 & 2.3997 & -4.6000 \\ \mathrm{H} & -1.0404 & 0.9194 & -5.0514 \\ \mathrm{H} & 0.6231 & 1.1850 & -5.6397 \\ \mathrm{C} & 0.3350 & -0.2451 & 3.1329 \\ \mathrm{O} & 1.1695 & 0.6345 & 3.1833 \\ \mathrm{C} & -0.1144 & -0.9282 & 4.4245 \\ \mathrm{C} & 0.8574 & -0.7327 & 5.5670 \\ \mathrm{H} & 0.4750 & -1.1891 & 6.4814 \\ \mathrm{H} & 1.0341 & 0.3306 & 5.7349 \\ \mathrm{H} & 1.8130 & -1.2004 & 5.3080 \\ \mathrm{C} & 0.3596 & -3.0813 & -1.6758 \\ \mathrm{H} & 1.4476 & -3.0324 & -1.5557 \\ \mathrm{H} & 0.1225 & -4.0098 & -2.2024 \\ -0.0904 & -3.1285 & -0.6802 \\ -0.3434 & -1.9793 & 4.2394 \\ \mathrm{H} & -1.8622 & -0.1223 & 4.8594\end{array}$


S19b-M0027

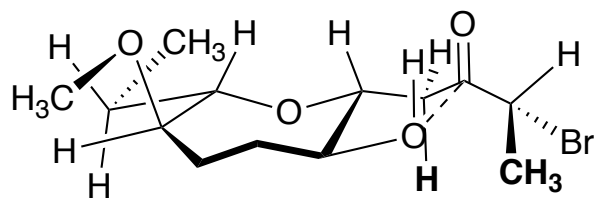

\begin{tabular}{lrrr}
$\mathrm{C}$ & 0.1813 & 2.0336 & -0.0979 \\
$\mathrm{H}$ & -0.4046 & 2.9651 & -0.0242 \\
$\mathrm{C}$ & -0.7902 & 0.9082 & -0.5013 \\
$\mathrm{H}$ & -0.7900 & 0.8730 & -1.6026 \\
$\mathrm{O}$ & -0.4224 & -0.3619 & 0.0110 \\
$\mathrm{C}$ & 0.8968 & -0.7980 & -0.2879 \\
$\mathrm{H}$ & 1.3211 & -0.1982 & -1.1032 \\
$\mathrm{C}$ & 1.7454 & -0.6117 & 0.9793 \\
$\mathrm{C}$ & 2.0785 & 0.8556 & 1.2098 \\
$\mathrm{C}$ & -2.2082 & 1.1615 & 0.0044 \\
$\mathrm{H}$ & -2.1679 & 1.3960 & 1.0761 \\
$\mathrm{H}$ & -2.7556 & 0.2174 & -0.0874 \\
$\mathrm{O}$ & 1.1106 & 2.1899 & -1.1581 \\
$\mathrm{C}$ & 0.8317 & -2.2519 & -0.7802 \\
$\mathrm{H}$ & 1.8395 & -2.5490 & -1.0865 \\
$\mathrm{O}$ & 2.9611 & -1.3233 & 0.8975 \\
$\mathrm{H}$ & 2.6288 & 0.9242 & 2.1535 \\
$\mathrm{H}$ & 2.7657 & 1.1699 & 0.4155 \\
$\mathrm{C}$ & 0.8680 & 1.7947 & 1.2580 \\
$\mathrm{H}$ & 0.1187 & 1.4119 & 1.9622 \\
$\mathrm{H}$ & 1.1964 & 2.7614 & 1.6555 \\
$\mathrm{H}$ & 1.1358 & -0.9743 & 1.8206 \\
$\mathrm{H}$ & 0.1772 & -2.3024 & -1.6538 \\
$\mathrm{H}$ & 2.7437 & -2.2424 & 1.1274 \\
$\mathrm{C}$ & 1.8828 & 3.3619 & -1.0606 \\
$\mathrm{H}$ & 1.2398 & 4.2482 & -0.9558 \\
$\mathrm{H}$ & 2.4582 & 3.4446 & -1.9841 \\
$\mathrm{H}$ & 2.5784 & 3.3327 & -0.2116 \\
$\mathrm{C}$ & 0.3893 & -3.2186 & 0.2973 \\
$\mathrm{O}$ & 1.0880 & -3.4544 & 1.2635 \\
$\mathrm{C}$ & -0.9654 & -3.9069 & 0.1893 \\
$\mathrm{C}$ & -2.1383 & -2.9498 & 0.0766 \\
$\mathrm{H}$ & -3.0743 & -3.5104 & 0.0305 \\
$\mathrm{H}$ & -2.0526 & -2.3183 & -0.8102 \\
$\mathrm{H}$ & -2.1499 & -2.2933 & 0.9527 \\
$\mathrm{C}$ & -2.9458 & 2.2584 & -0.7591 \\
$\mathrm{H}$ & -3.0042 & 2.0187 & -1.8256 \\
-2.4540 & 3.2314 & -0.6644 \\
$\mathrm{H}$ & -3.9664 & 2.3698 & -0.3838 \\
$\mathrm{H}$ & -0.8881 & -5.0857 & -1.3852 \\
\hline
\end{tabular}


S19b-M0113

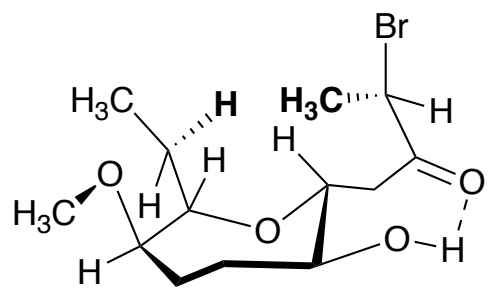

$\begin{array}{lrrr}\mathrm{C} & -0.5980 & -0.2550 & 2.3797 \\ \mathrm{H} & -0.7027 & -0.6884 & 3.3891 \\ \mathrm{C} & -0.4857 & -1.4231 & 1.3819 \\ \mathrm{H} & -1.5075 & -1.6452 & 1.0353 \\ \mathrm{O} & 0.3371 & -1.1266 & 0.2692 \\ \mathrm{C} & -0.0538 & -0.0147 & -0.5184 \\ \mathrm{H} & -1.0972 & 0.2604 & -0.3122 \\ \mathrm{C} & 0.8818 & 1.1473 & -0.1643 \\ \mathrm{C} & 0.6373 & 1.6984 & 1.2381 \\ \mathrm{C} & 0.1162 & -2.6634 & 2.0336 \\ \mathrm{H} & -0.5195 & -2.9480 & 2.8800 \\ \mathrm{H} & 1.0995 & -2.3999 & 2.4452 \\ \mathrm{O} & -1.7946 & 0.4443 & 2.0782 \\ \mathrm{C} & 0.0773 & -0.4122 & -1.9850 \\ \mathrm{H} & -0.4517 & -1.3614 & -2.1362 \\ \mathrm{O} & 0.8152 & 2.1886 & -1.1271 \\ \mathrm{H} & 1.4052 & 2.4538 & 1.4339 \\ \mathrm{H} & -0.3285 & 2.2210 & 1.2263 \\ \mathrm{C} & 0.6340 & 0.6660 & 2.3715 \\ \mathrm{H} & 1.5337 & 0.0409 & 2.3210 \\ \mathrm{H} & 0.6812 & 1.2019 & 3.3258 \\ \mathrm{H} & 1.9038 & 0.7539 & -0.2334 \\ \mathrm{H} & 1.1253 & -0.5968 & -2.2457 \\ \mathrm{H} & -0.1053 & 2.4970 & -1.1585 \\ \mathrm{C} & -2.1844 & 1.3505 & 3.0808 \\ \mathrm{H} & -2.2457 & 0.8525 & 4.0595 \\ \mathrm{H} & -3.1722 & 1.7253 & 2.8076 \\ \mathrm{H} & -1.4942 & 2.2010 & 3.1660 \\ \mathrm{C} & -0.5425 & 0.6094 & -2.9137 \\ \mathrm{O} & -1.5127 & 1.2661 & -2.6002 \\ \mathrm{C} & 0.0871 & 0.8454 & -4.2824 \\ \mathrm{C} & 1.4266 & 1.5597 & -4.1856 \\ \mathrm{H} & 1.8350 & 1.7293 & -5.1839 \\ \mathrm{H} & 2.1453 & 0.9771 & -3.6044 \\ \mathrm{H} & 1.2843 & 2.5180 & -3.6785 \\ \mathrm{C} & 0.2524 & -3.8301 & 1.0606 \\ \mathrm{H} & 0.9112 & -3.5636 & 0.2318 \\ \mathrm{H} & -0.7234 & -4.1008 & 0.6429 \\ \mathrm{H} & 0.6614 & -4.7119 & 1.5612 \\ \mathrm{H} & & 1.4095 & -4.8783 \\ \mathrm{H} & & -0.8762 & -5.2115\end{array}$


S19b-M0021

ir

$\begin{array}{lrrr}\mathrm{C} & 0.7761 & 0.8741 & -2.0891 \\ \mathrm{H} & 0.3182 & 1.3251 & -2.9861 \\ \mathrm{C} & -0.3647 & 0.4554 & -1.1312 \\ \mathrm{H} & -0.5429 & -0.6246 & -1.2547 \\ \mathrm{O} & -0.0383 & 0.6940 & 0.2252 \\ \mathrm{C} & 1.0472 & -0.0727 & 0.7244 \\ \mathrm{H} & 1.2839 & -0.8918 & 0.0332 \\ \mathrm{C} & 2.2456 & 0.8589 & 0.8499 \\ \mathrm{C} & 2.7732 & 1.3017 & -0.5132 \\ \mathrm{C} & -1.6621 & 1.2055 & -1.3990 \\ \mathrm{H} & -1.9605 & 1.0105 & -2.4363 \\ \mathrm{H} & -1.4749 & 2.2844 & -1.3174 \\ \mathrm{O} & 1.5533 & -0.2394 & -2.4986 \\ \mathrm{C} & 0.6059 & -0.6448 & 2.0626 \\ \mathrm{H} & 0.4426 & 0.1621 & 2.7868 \\ \mathrm{O} & 3.2381 & 0.1543 & 1.5762 \\ \mathrm{H} & 3.5604 & 2.0497 & -0.3465 \\ \mathrm{H} & 3.2450 & 0.4361 & -0.9894 \\ \mathrm{C} & 1.7390 & 1.8994 & -1.4778 \\ \mathrm{H} & 1.1549 & 2.6835 & -0.9820 \\ \mathrm{H} & 2.2829 & 2.3694 & -2.3032 \\ \mathrm{H} & 1.9013 & 1.7404 & 1.4169 \\ \mathrm{H} & 1.3842 & -1.2931 & 2.4781 \\ \mathrm{H} & 4.0437 & 0.6894 & 1.5830 \\ \mathrm{C} & 0.9416 & -1.0115 & -3.5017 \\ \mathrm{H} & 0.7671 & -0.4131 & -4.4079 \\ \mathrm{H} & -0.0179 & -1.4351 & -3.1753 \\ \mathrm{H} & 1.6223 & -1.8310 & -3.7382 \\ \mathrm{C} & -0.6731 & -1.4363 & 1.8680 \\ \mathrm{O} & -0.9007 & -2.0656 & 0.8598 \\ \mathrm{C} & -1.7254 & -1.3842 & 2.9755 \\ \mathrm{C} & -2.9745 & -2.1702 & 2.6484 \\ \mathrm{H} & -2.7256 & -3.2112 & 2.4354 \\ \mathrm{H} & -3.4348 & -1.7419 & 1.7528 \\ \mathrm{H} & -3.6865 & -2.1267 & 3.4743 \\ \mathrm{H} & -2.7732 & 0.7843 & -0.4415 \\ \mathrm{H} & -2.9292 & -0.2994 & -0.4921 \\ -3.7172 & 1.2802 & -0.6836 \\ \mathrm{H} & -2.5031 & 1.0365 & 0.5871 \\ \mathrm{H} & -0.8799 & -2.0650 & 4.6233\end{array}$


S19b-M0023

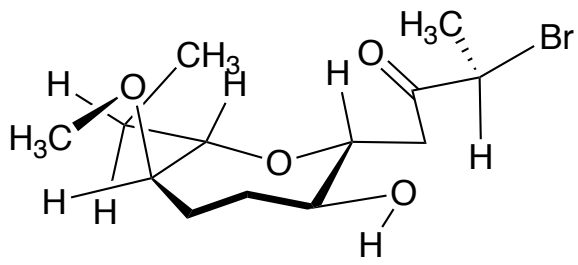

$\begin{array}{lrrr}\mathrm{C} & -1.6448 & -1.4534 & 0.6839 \\ \mathrm{H} & -2.5964 & -1.4221 & 1.2427 \\ \mathrm{C} & -0.9622 & -0.0762 & 0.8169 \\ \mathrm{H} & -0.1880 & -0.1733 & 1.5925 \\ \mathrm{O} & -0.3535 & 0.3448 & -0.3899 \\ \mathrm{C} & 0.7481 & -0.4435 & -0.8149 \\ \mathrm{H} & 1.0960 & -1.0847 & 0.0055 \\ \mathrm{C} & 0.2786 & -1.3023 & -1.9822 \\ \mathrm{C} & -0.7391 & -2.3579 & -1.5509 \\ \mathrm{C} & -1.9354 & 1.0276 & 1.2084 \\ \mathrm{H} & -2.4140 & 0.7434 & 2.1526 \\ \mathrm{H} & -2.7284 & 1.0923 & 0.4515 \\ \mathrm{O} & -0.7943 & -2.3942 & 1.3177 \\ \mathrm{C} & 1.8549 & 0.5244 & -1.2080 \\ \mathrm{H} & 1.5573 & 1.1068 & -2.0877 \\ \mathrm{O} & 1.4347 & -1.9086 & -2.5330 \\ \mathrm{H} & -1.0934 & -2.8759 & -2.4524 \\ \mathrm{H} & -0.2027 & -3.0967 & -0.9442 \\ \mathrm{C} & -1.9560 & -1.8401 & -0.7726 \\ \mathrm{H} & -2.3947 & -0.9703 & -1.2759 \\ \mathrm{H} & -2.7245 & -2.6209 & -0.7717 \\ \mathrm{H} & -0.1882 & -0.6183 & -2.7110 \\ \mathrm{H} & 2.7653 & -0.0217 & -1.4751 \\ \mathrm{H} & 1.1549 & -2.5056 & -3.2409 \\ \mathrm{C} & -1.3933 & -3.6486 & 1.5270 \\ \mathrm{H} & -1.5615 & -4.1935 & 0.5875 \\ \mathrm{H} & -2.3576 & -3.5442 & 2.0458 \\ \mathrm{H} & -0.7124 & -4.2292 & 2.1518 \\ \mathrm{C} & 2.1299 & 1.4482 & -0.0362 \\ \mathrm{O} & 2.1228 & 1.0599 & 1.1093 \\ \mathrm{C} & 2.3645 & 2.9288 & -0.3312 \\ \mathrm{C} & 2.5509 & 3.7646 & 0.9139 \\ \mathrm{H} & 2.7440 & 4.8071 & 0.6553 \\ \mathrm{H} & 3.3775 & 3.3786 & 1.5131 \\ \mathrm{H} & 1.6392 & 3.7061 & 1.5161 \\ \mathrm{C} & -1.2326 & 2.3741 & 1.3584 \\ \mathrm{H} & -1.9230 & 3.1475 & 1.7059 \\ \mathrm{H} & -0.8104 & 2.6918 & 0.4010 \\ -0.4106 & 2.2940 & 2.0786 \\ 1.5481 & 3.2944 & -0.9594 \\ \mathrm{H} & 3.9504 & 3.0471 & -1.4981\end{array}$


(1) (a) Ferrier, R. J.; Prasad, N. J. Chem. Soc., C. 1969, 570-575. (b) Shiozaki, M.; Hata, T.; Furukawa, Y. Tetrahedron Lett. 1989, 30, 3669-3670.

(2) Sakai, T.; Matsushita, S.; Arakawa, S.; Mori, K.; Tanimoto, M.; Tokumasu, A.; Yoshida, T.; Mori, Y. J. Am. Chem. Soc. 2015, 137, 14513-14516.

(3) Sakai, T.; Matsushita, S.; Arakawa, S.; Kawai, A.; Mori, Y. Tetrahedron Lett. 2014, 55, 6557-6560.

(4) Mori, Y.; Furuta, H.; Takase, T.; Mitsuoka, S.; Furukawa, H. Tetrahedron Lett. 1999, 40, 80198022 .

(5) Sakai, T.; Asano, H.; Furukawa, K.; Oshima, R.; Mori, Y. Org. Lett. 2014, 16, 2268-2271. 


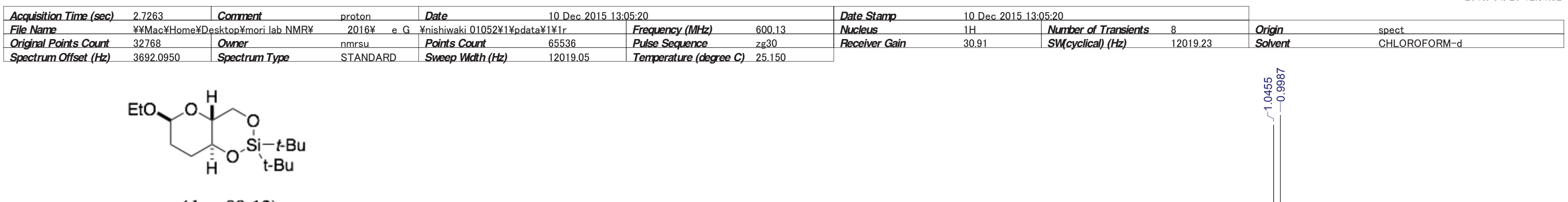

$(\mathrm{dr}=88: 12)$

1H NMR spectrum of S1

(600 MHz, CDCl $)_{3}$ 


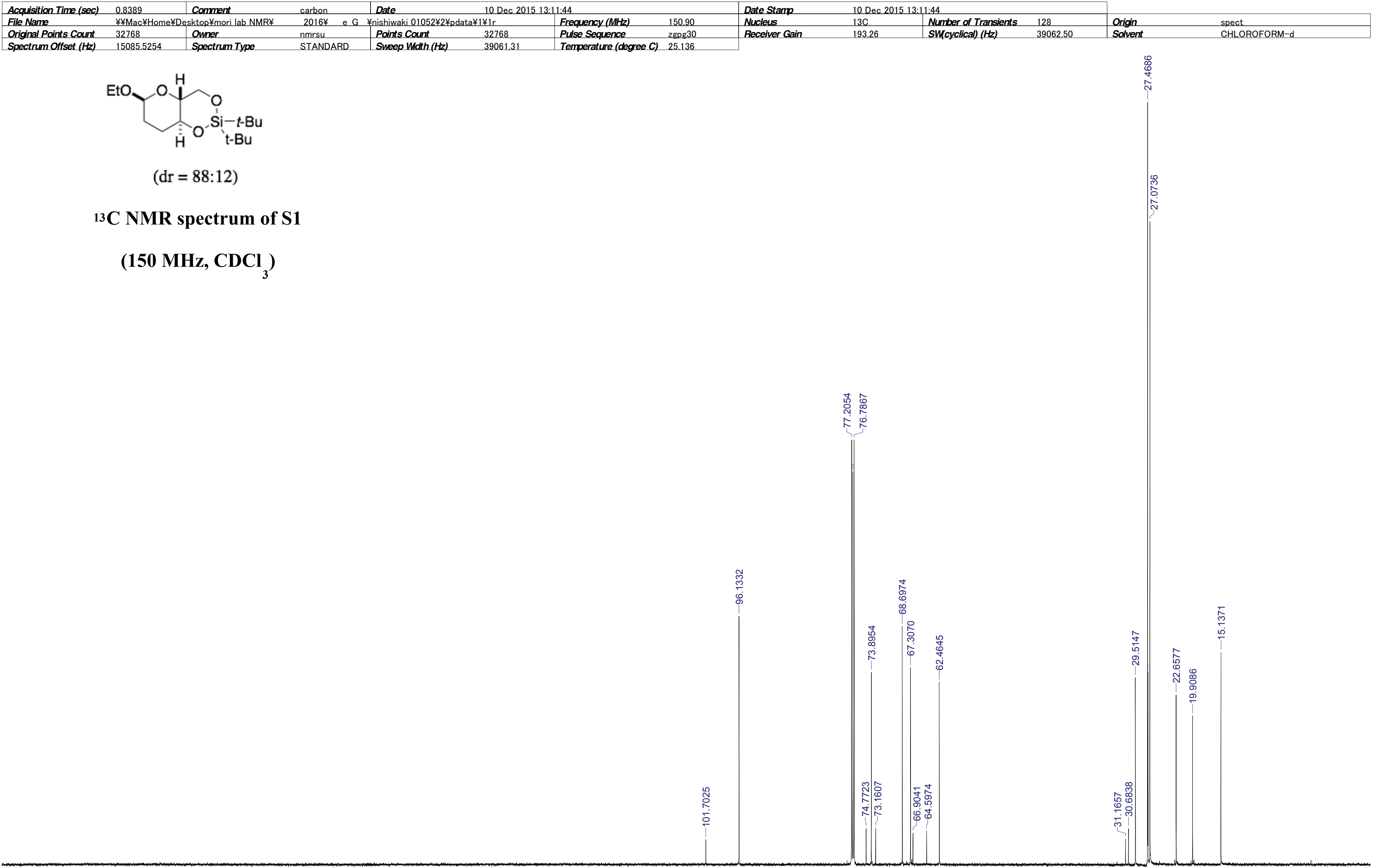

\section{(150 $\left.\mathrm{MHz}, \mathrm{CDCl}_{3}\right)$}

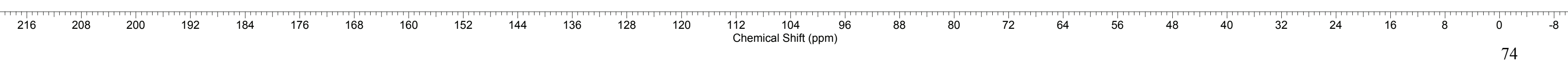


This report was created by ACD/NMR Processor Academic Edition. For more information go to www.acdlabs.com/nmrproc/

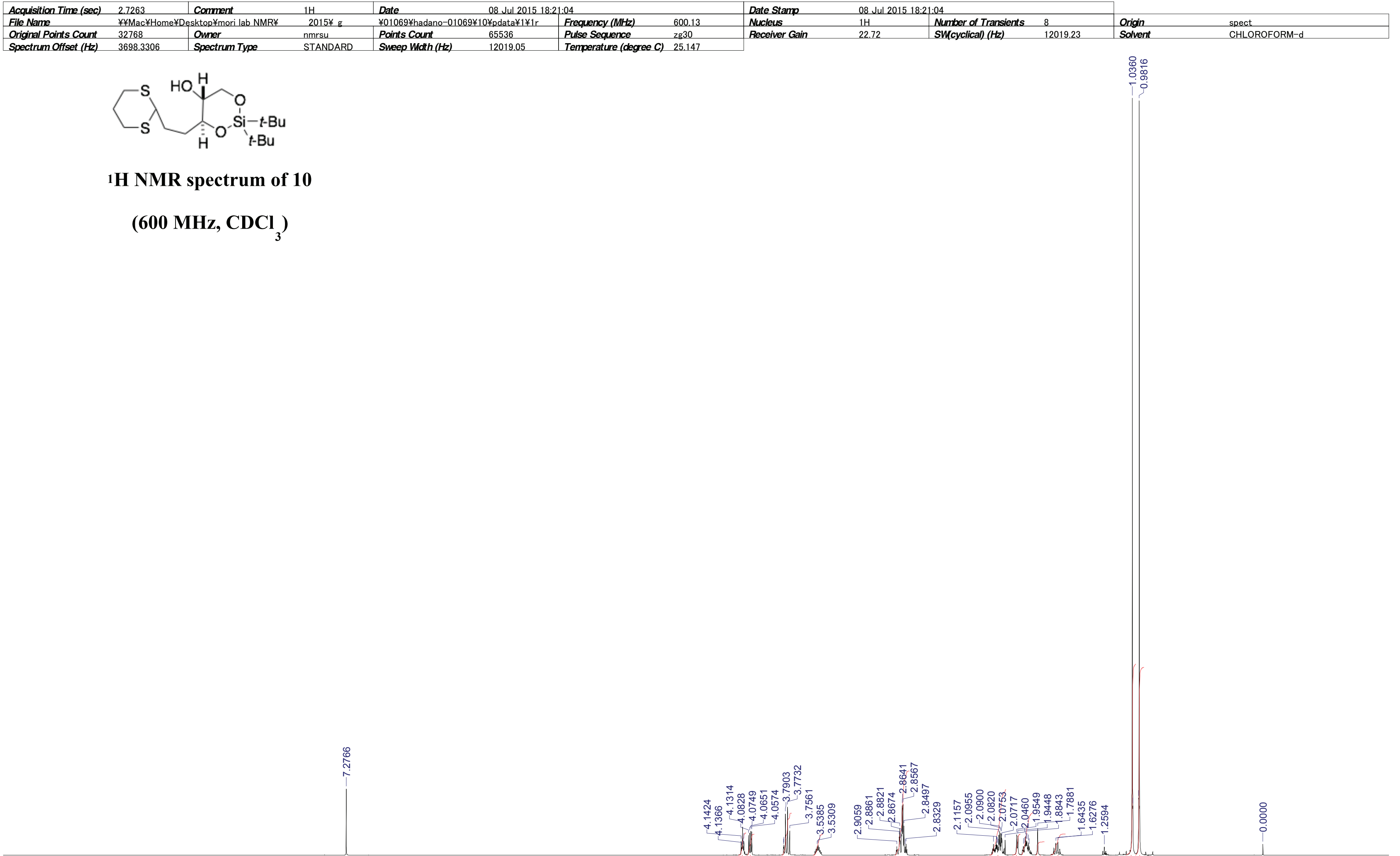

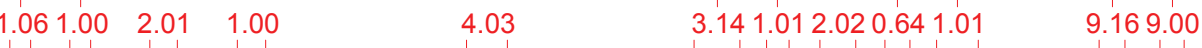


This report was created by ACD/NMR Processor Academic Edition. For more information go to www.acdlabs.com/nmrproc/

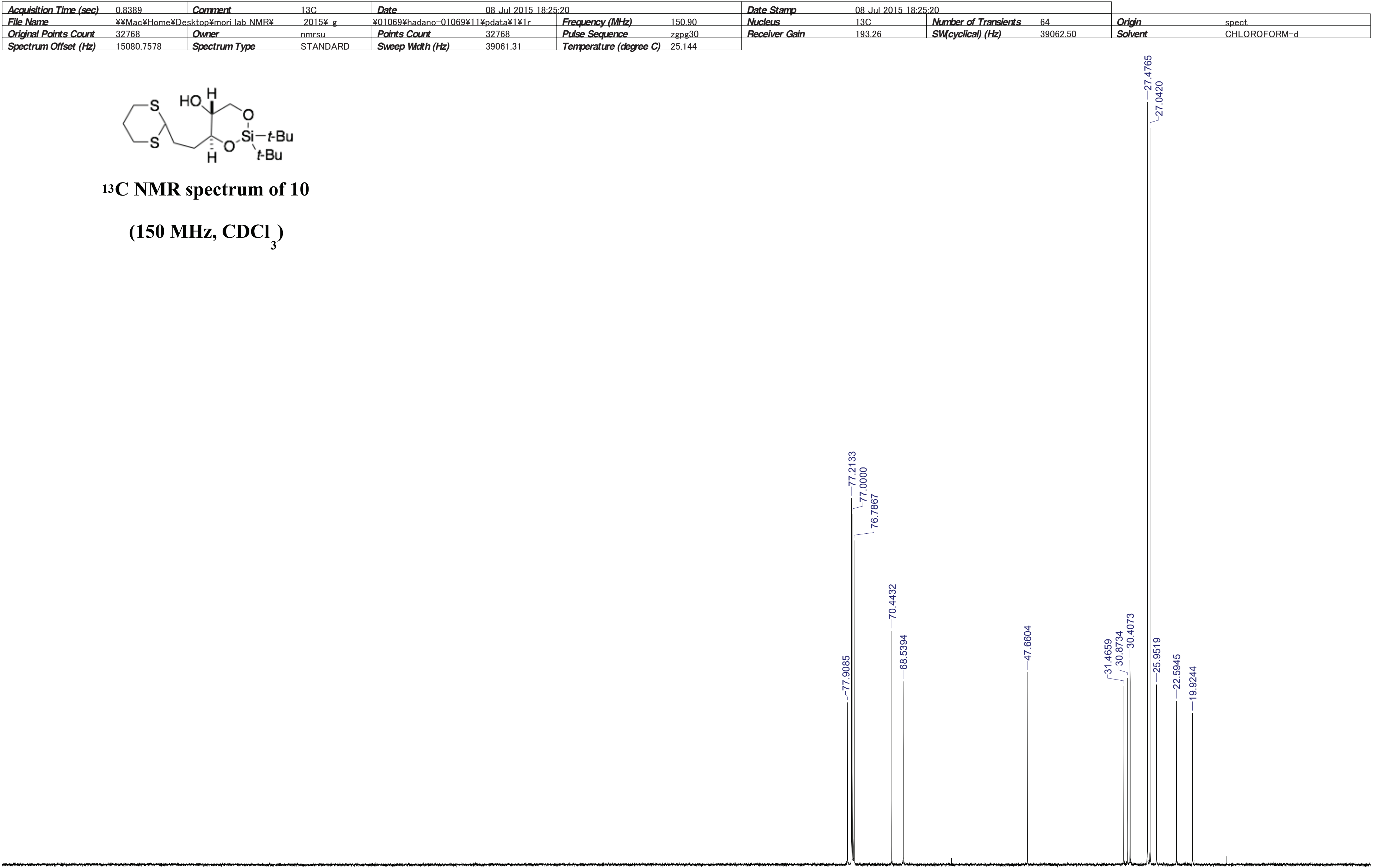

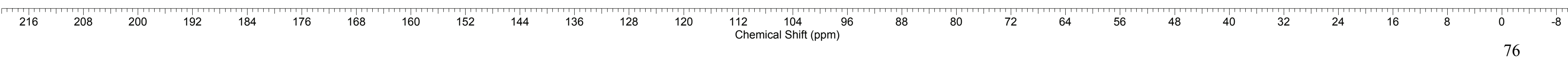


This report was created by ACD/NMR Processor Academic Edition. For more information go to www.acdlabs.com/nmrproc/

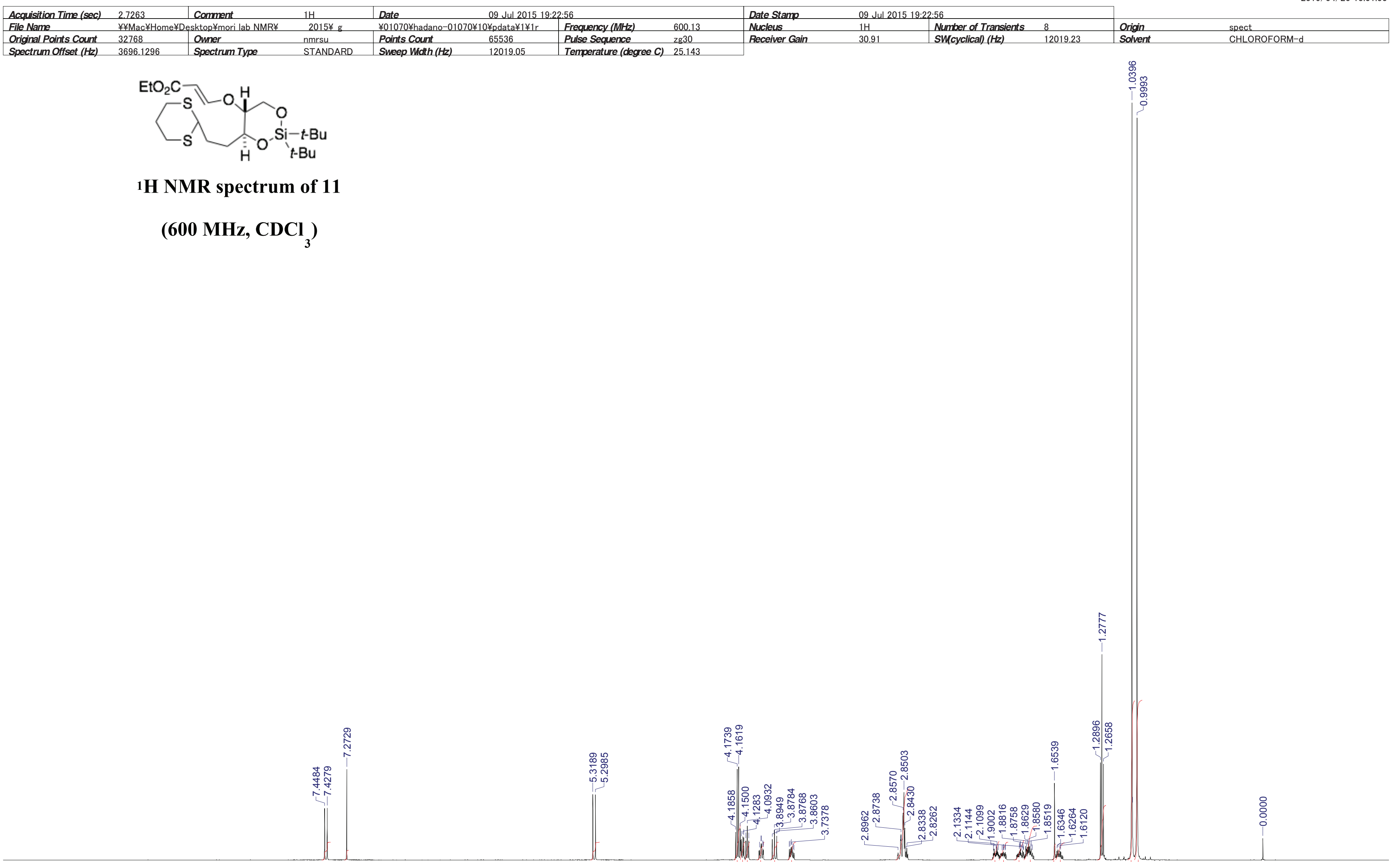

$\begin{array}{lllll}0.990 .54 & 0.98 & 2.011 .001 .001 .001 .001 .00 & 3.98 & 1.040 .991 .111 .900 .910 .953 .068 .918 .95\end{array}$

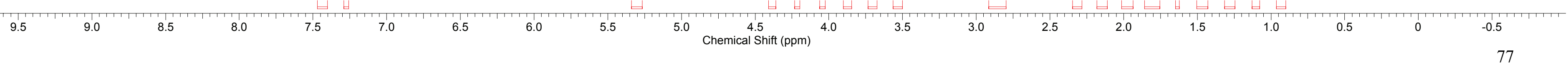


This report was created by ACD/NMR Processor Academic Edition. For more information go to www.acdlabs.com/nmrproc/

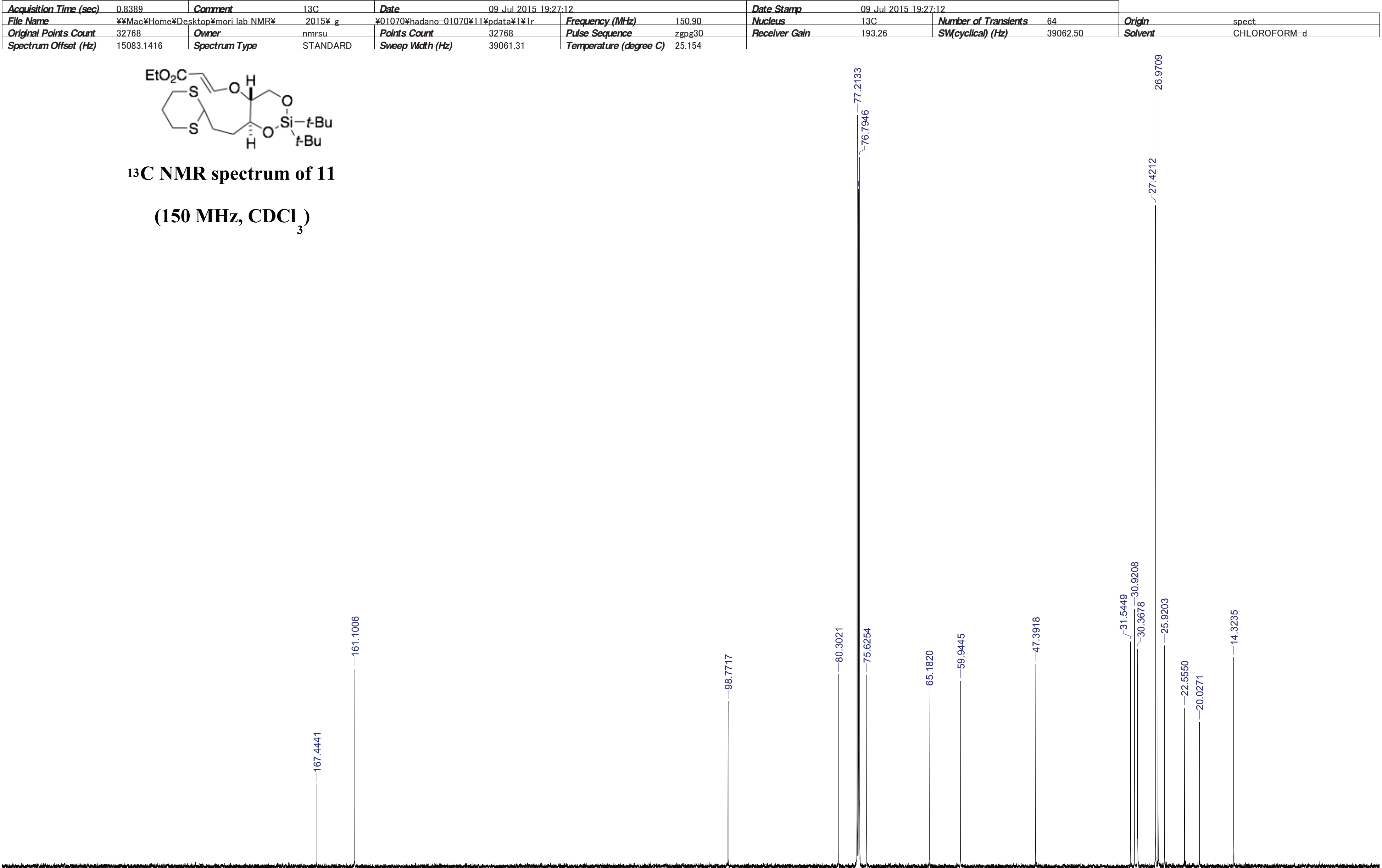


This report was created by ACD/NMR Processor Academic Edition. For more information go to www.acdlabs.com/nmrproc/

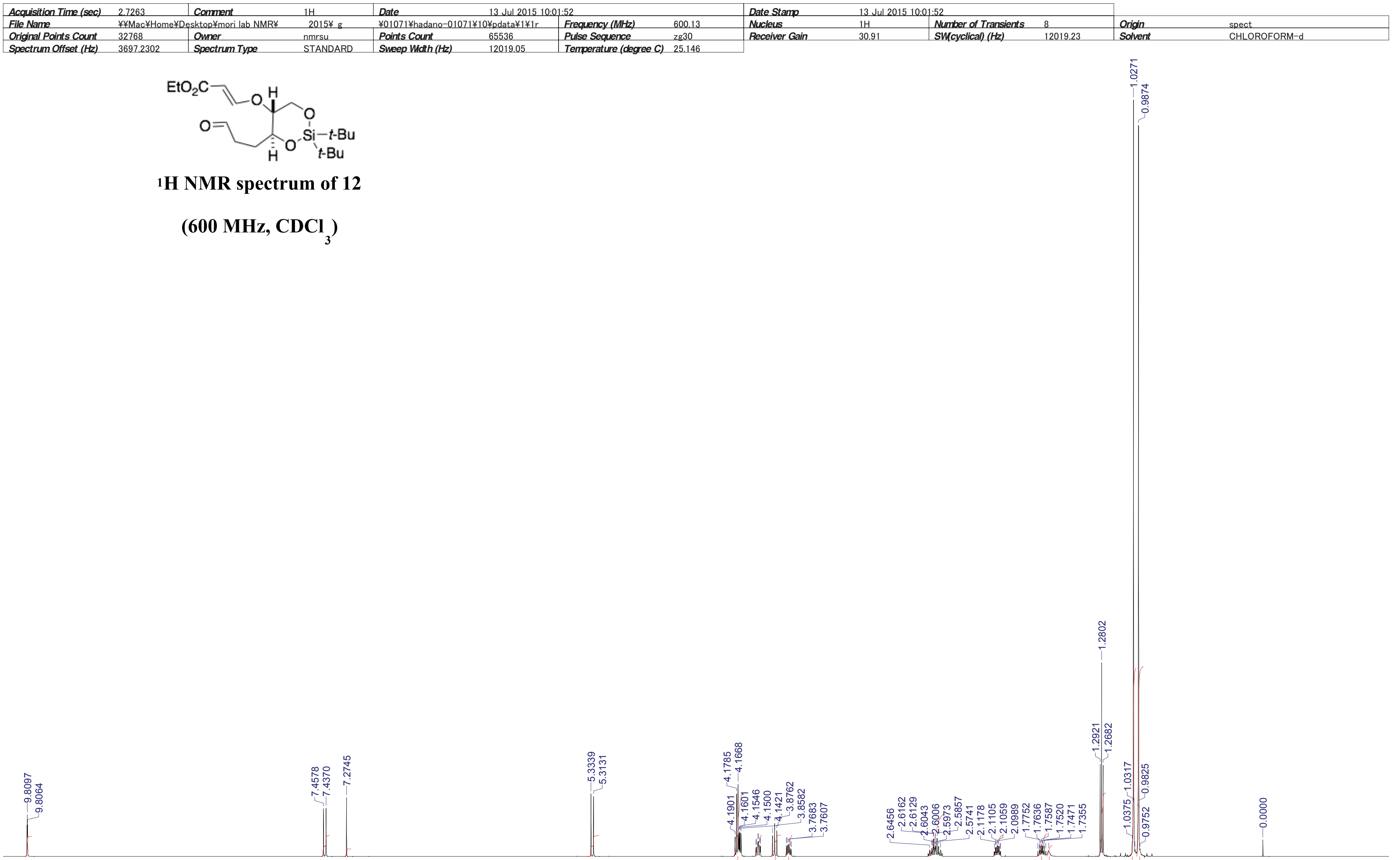


This report was created by ACD/NMR Processor Academic Edition. For more information go to www.acdlabs.com/nmrproc/
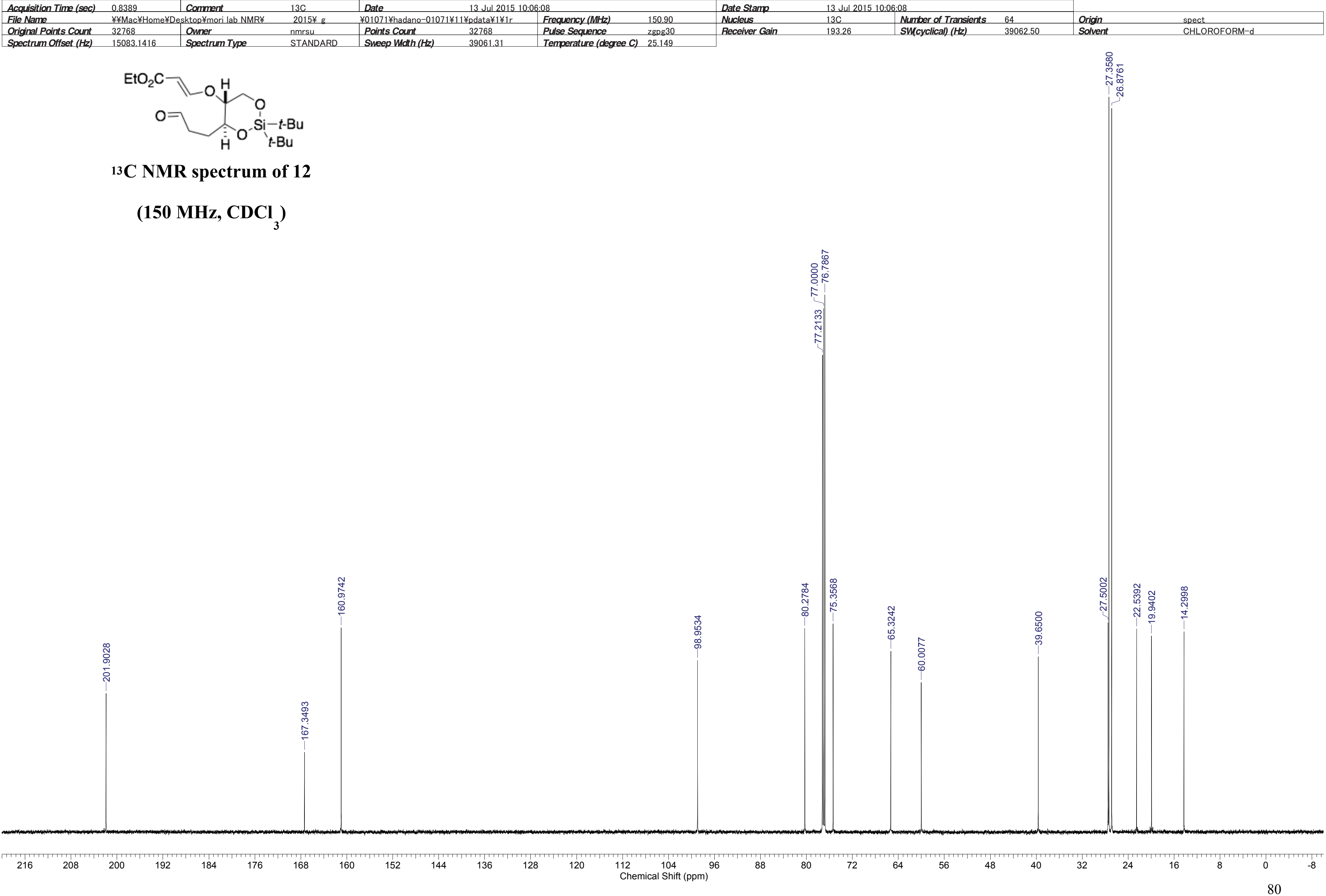
This report was created by ACD/NMR Processor Academic Edition. For more information go to www.acdlabs.com/nmrproc/

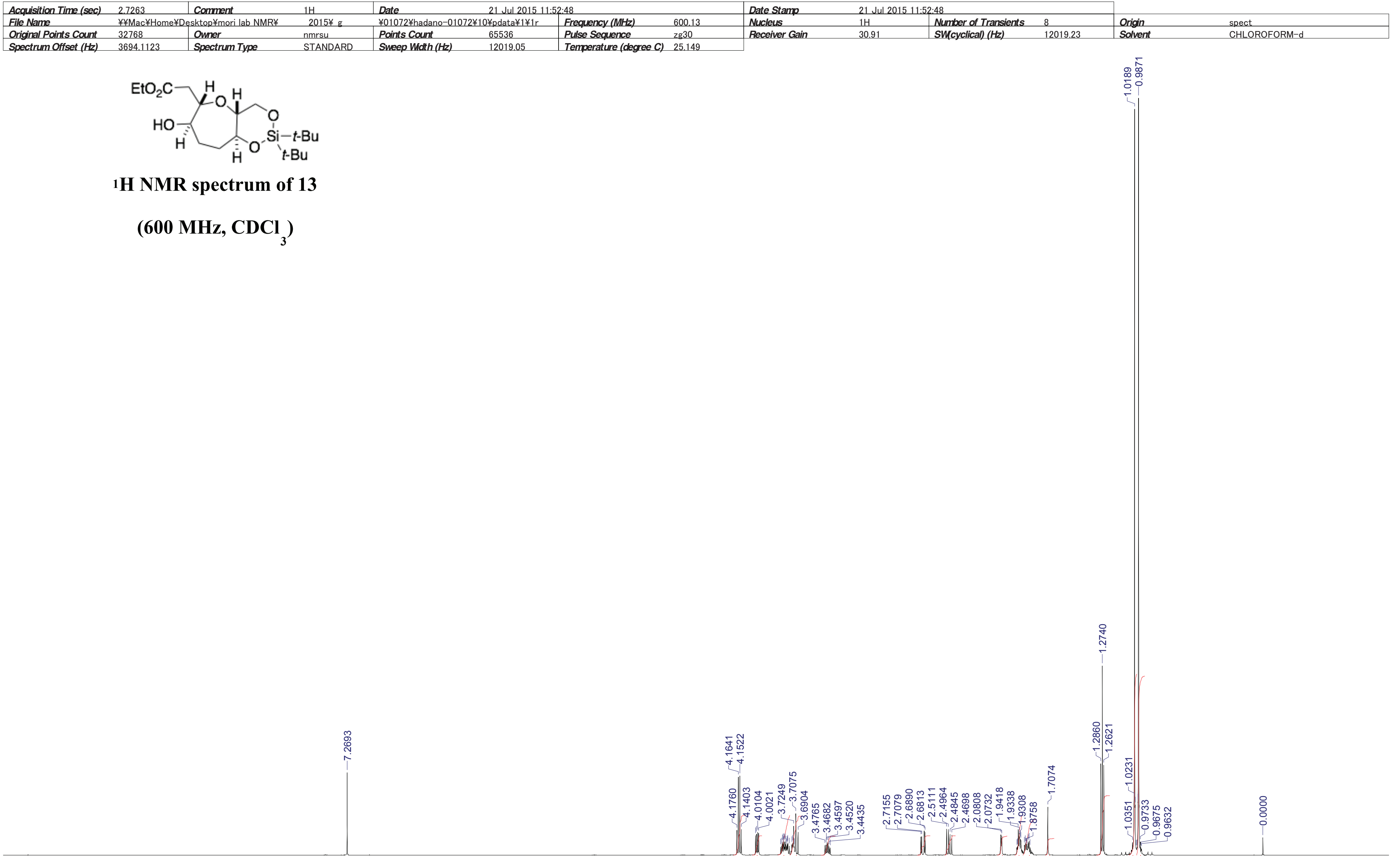

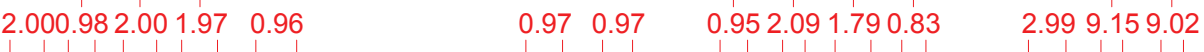


This report was created by ACD/NMR Processor Academic Edition. For more information go to www.acdlabs.com/nmrproc/

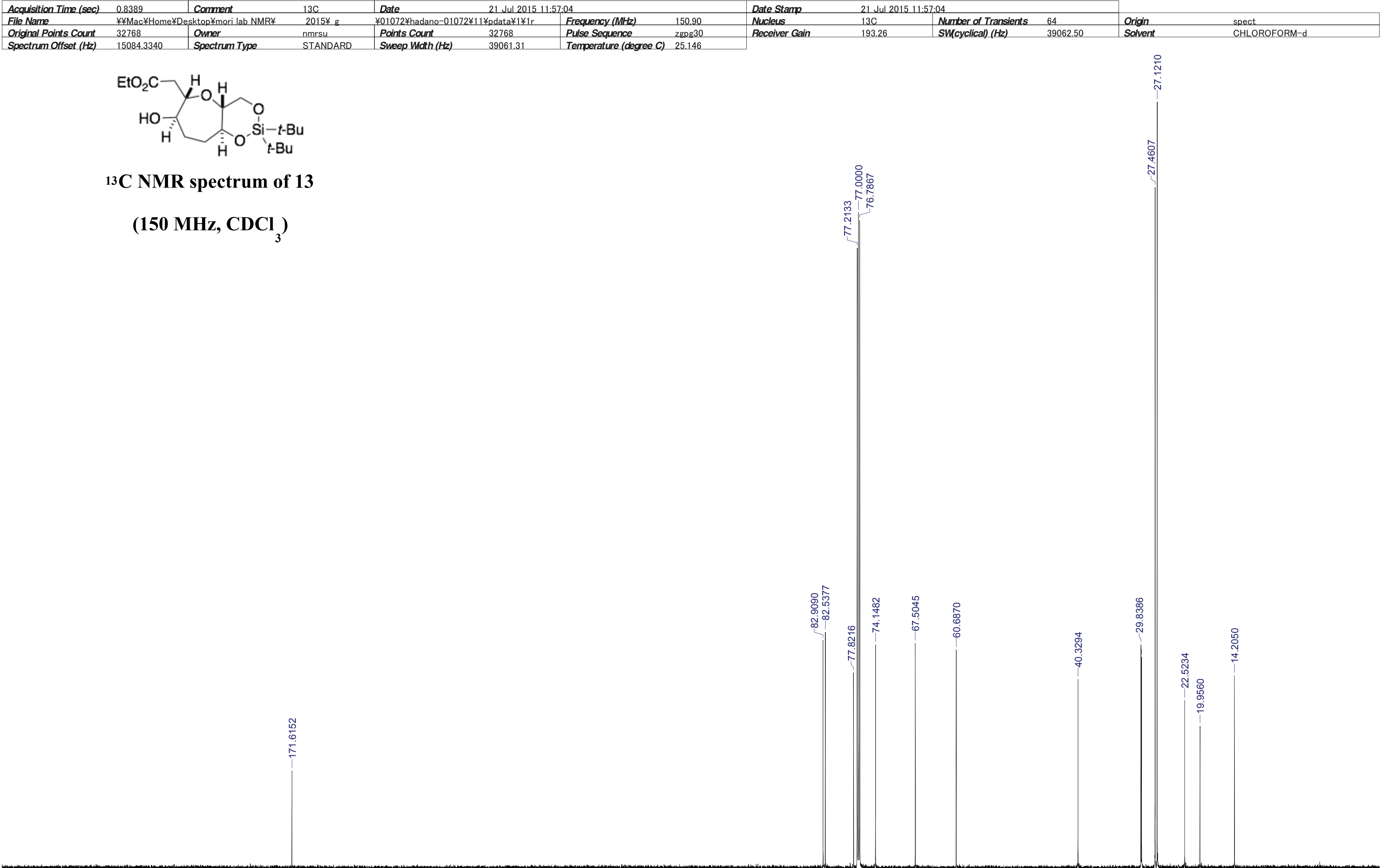


This report was created by ACD/NMR Processor Academic Edition. For more information go to www.acdlabs.com/nmrproc/

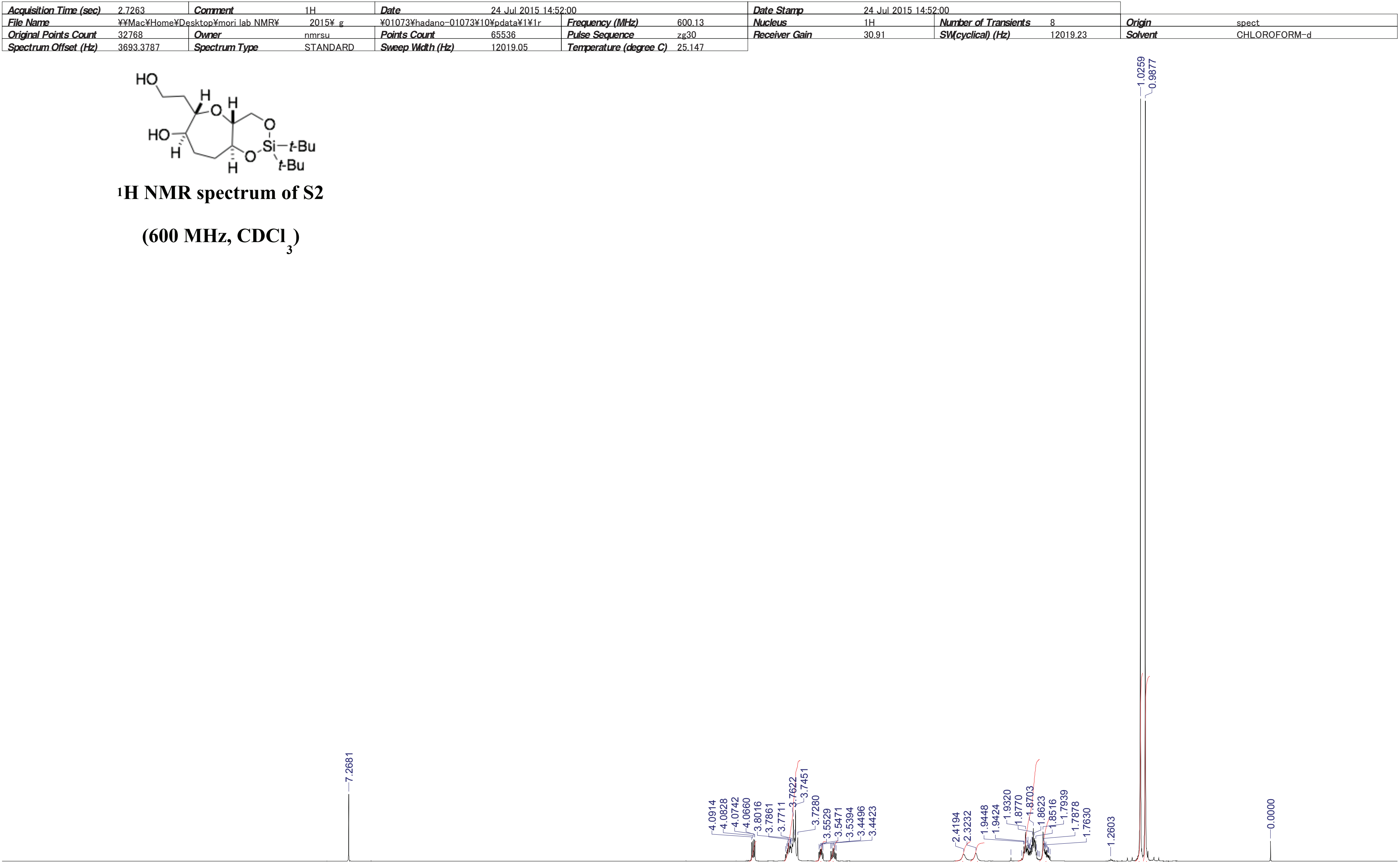

$0.98 \quad 5.010 .991 .00$

$0.980 .91 \quad 5.072 .22$

9.369 .19 
This report was created by ACD/NMR Processor Academic Edition. For more information go to www.acdlabs.com/nmrproc/

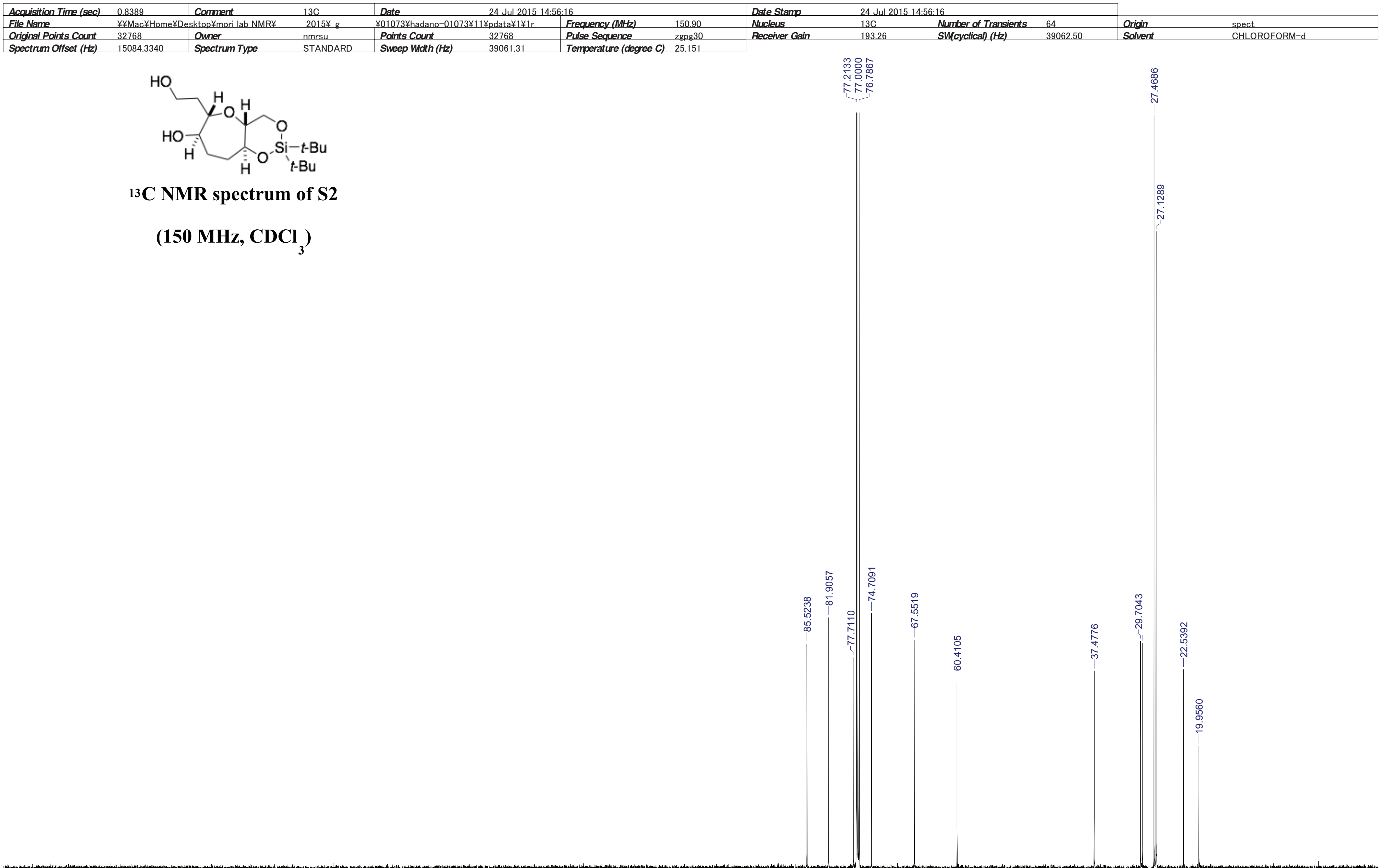

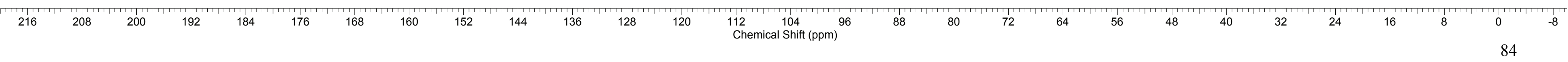


This report was created by ACD/NMR Processor Academic Edition. For more information go to www.acdlabs.com/nmrproc/

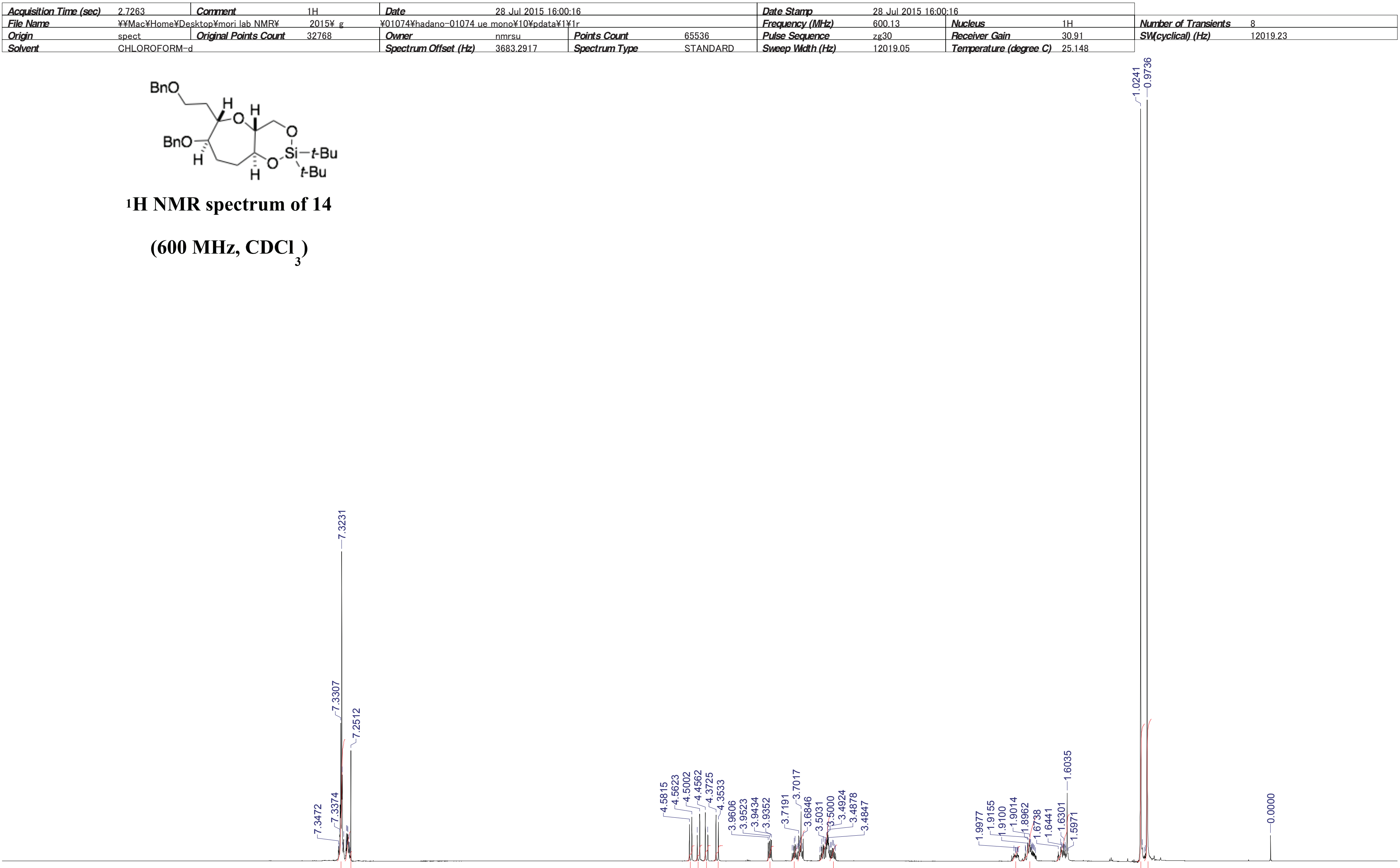

7.851 .910 .62

0.981 .031 .000 .990 .960 .961 .952 .921 .04

0.942 .903 .00

8.859 .17 
This report was created by ACD/NMR Processor Academic Edition. For more information go to www.acdlabs.com/nmrproc/

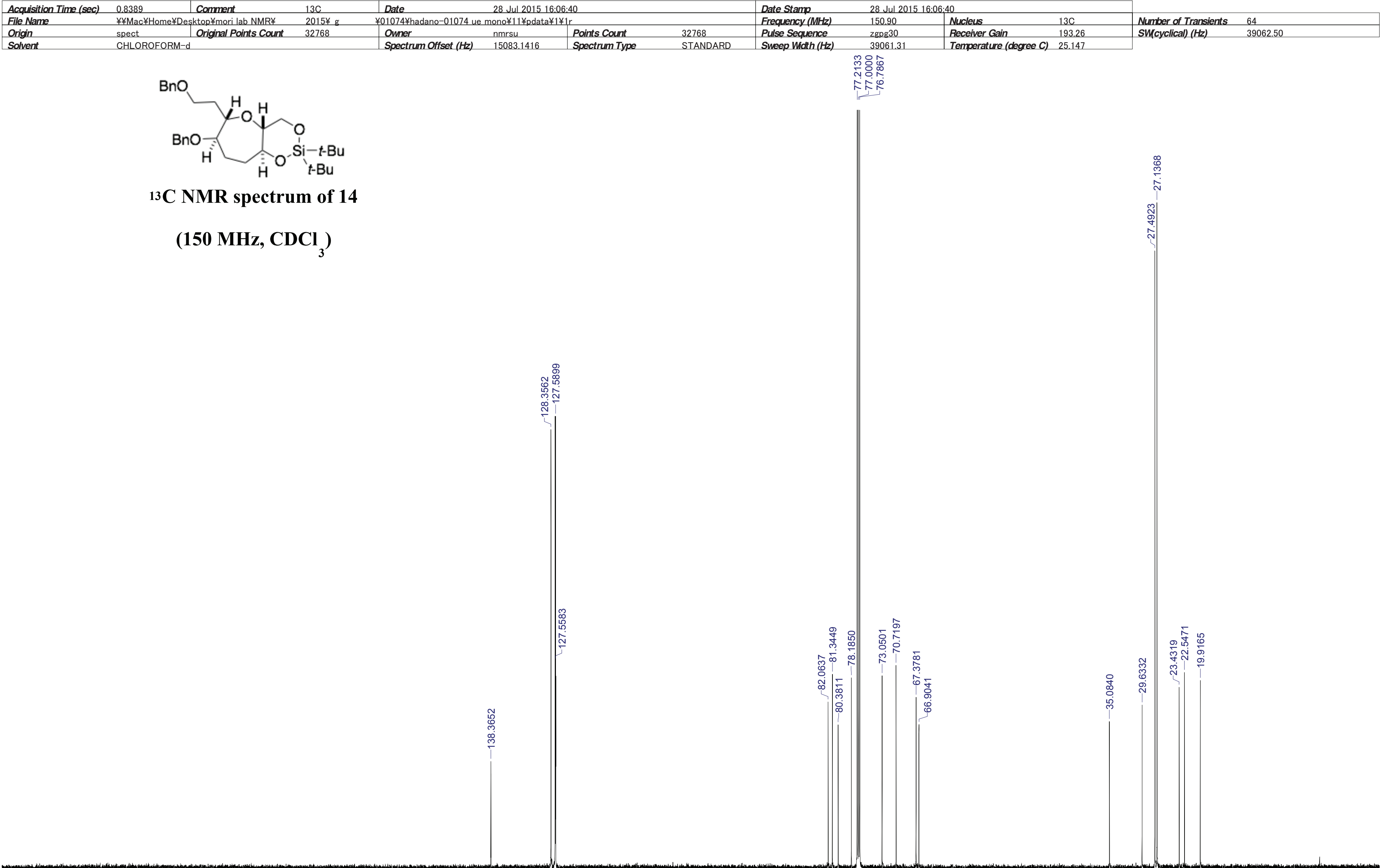


This report was created by ACD/NMR Processor Academic Edition. For more information go to www.acdlabs.com/nmrproc/

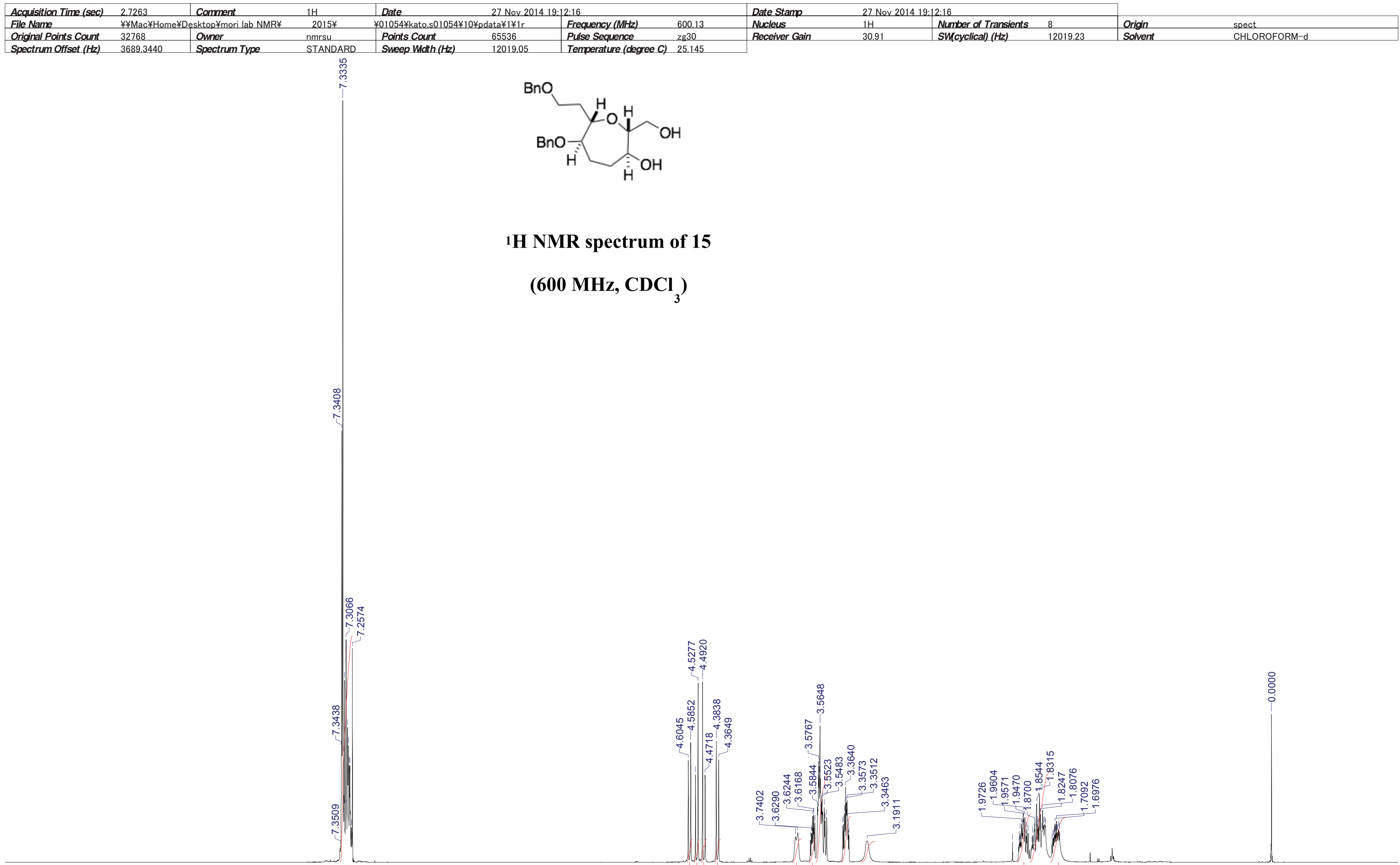

$1.000 .991 .021 .00 \quad 1.031 .064 .022 .010 .92$

2.124 .041 .99

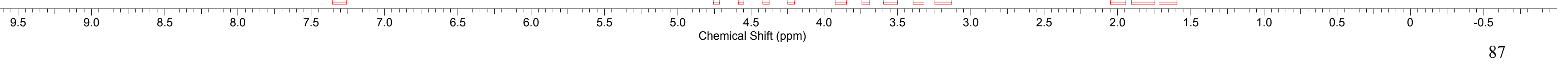


This report was created by ACD/NMR Processor Academic Edition. For more information go to www.acdlabs.com/nmrproc/

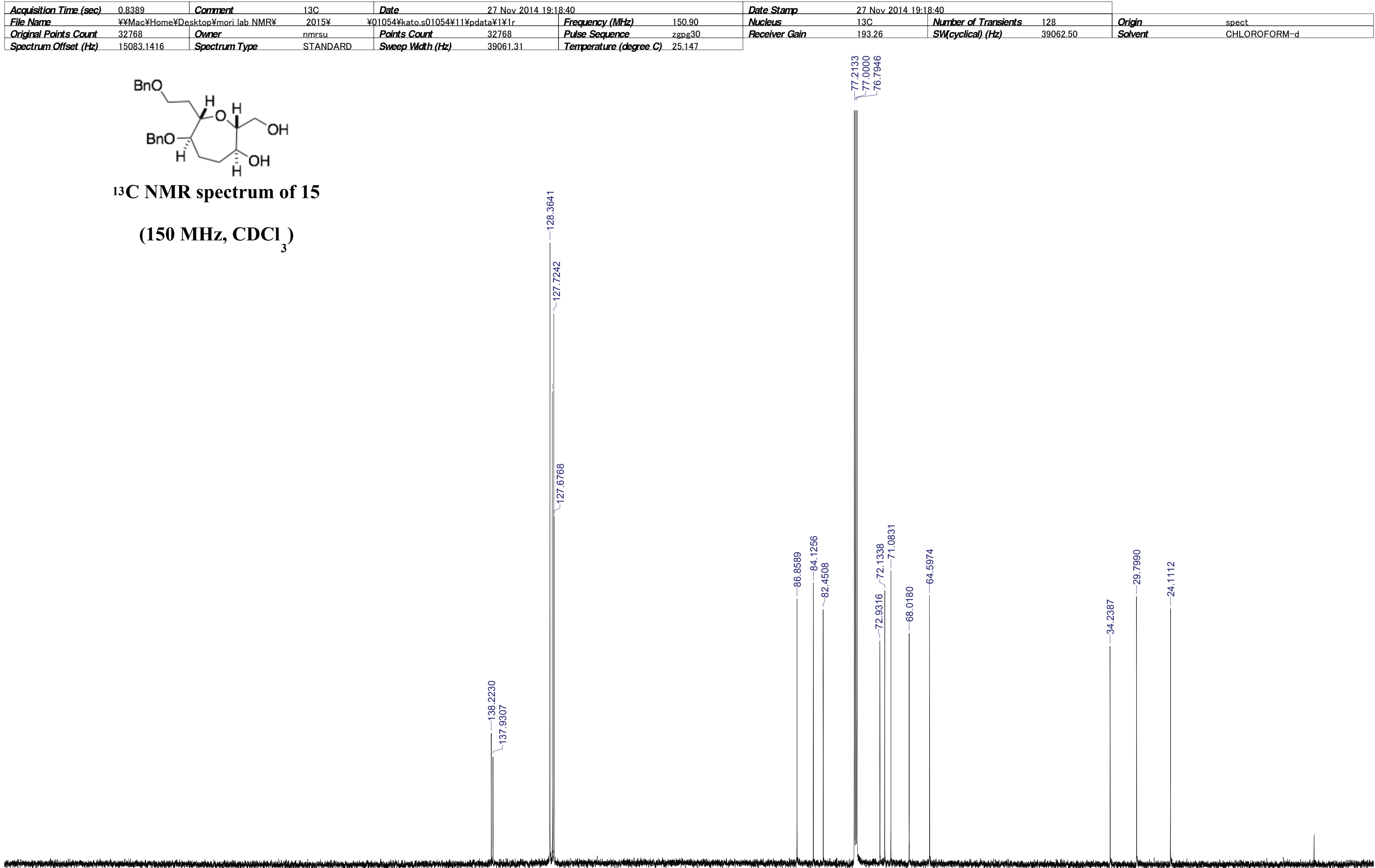


This report was created by ACD/NMR Processor Academic Edition. For more information go to www.acdlabs.com/nmrproc/

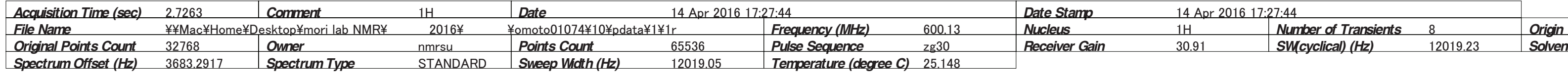

$\mathrm{BnO}$

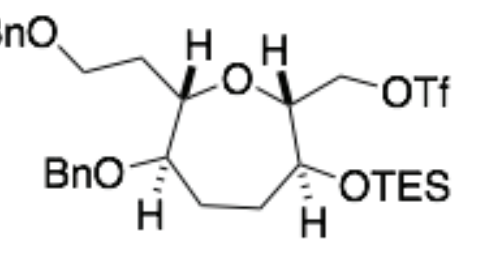

1H NMR spectrum of 8

(600 MHz, CDCl )

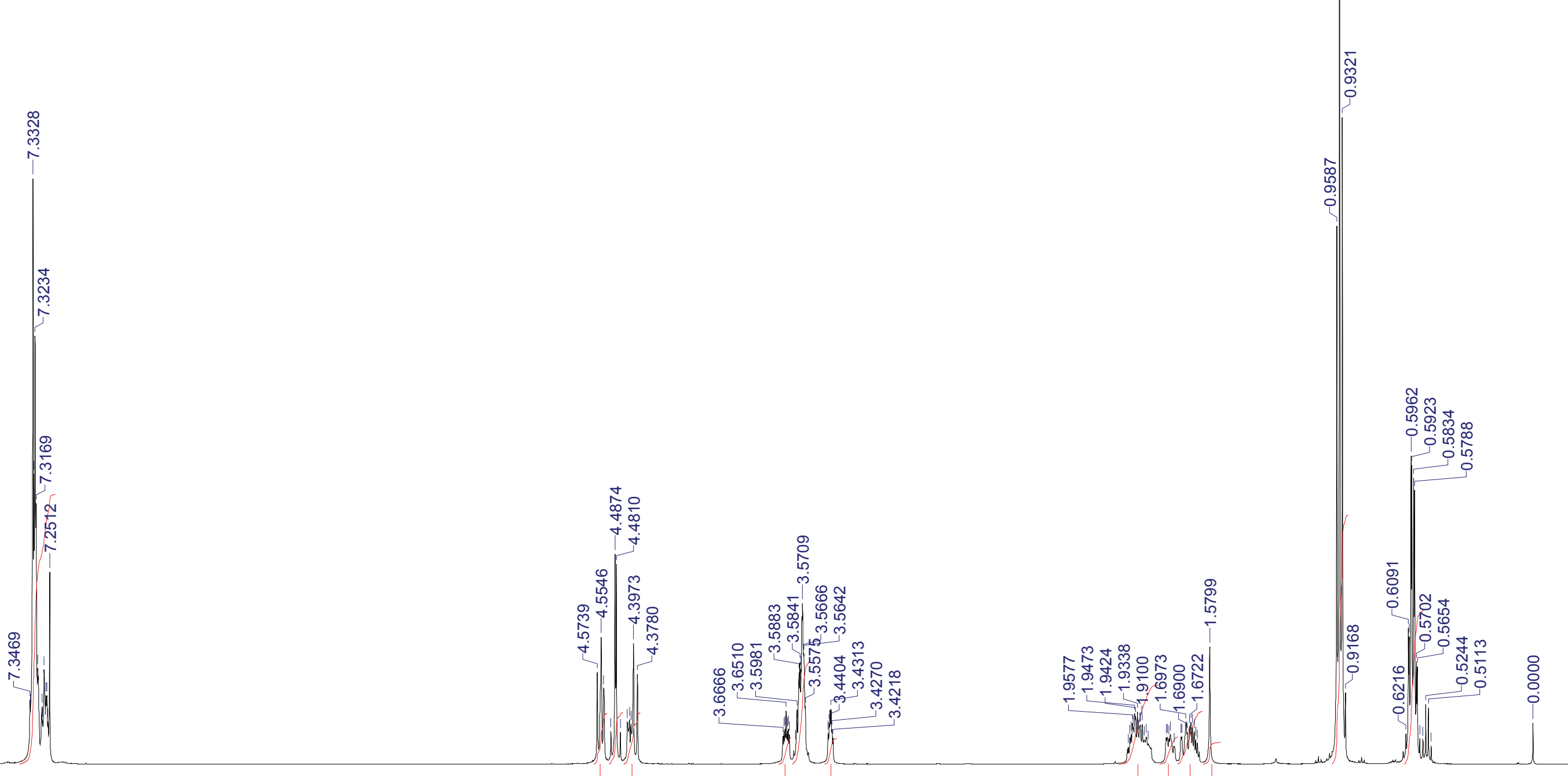

1.043 .960 .99

3.071 .052 .050 .85

$9.69 \quad 6.02$ 
This report was created by ACD/NMR Processor Academic Edition. For more information go to www.acdlabs.com/nmrproc/
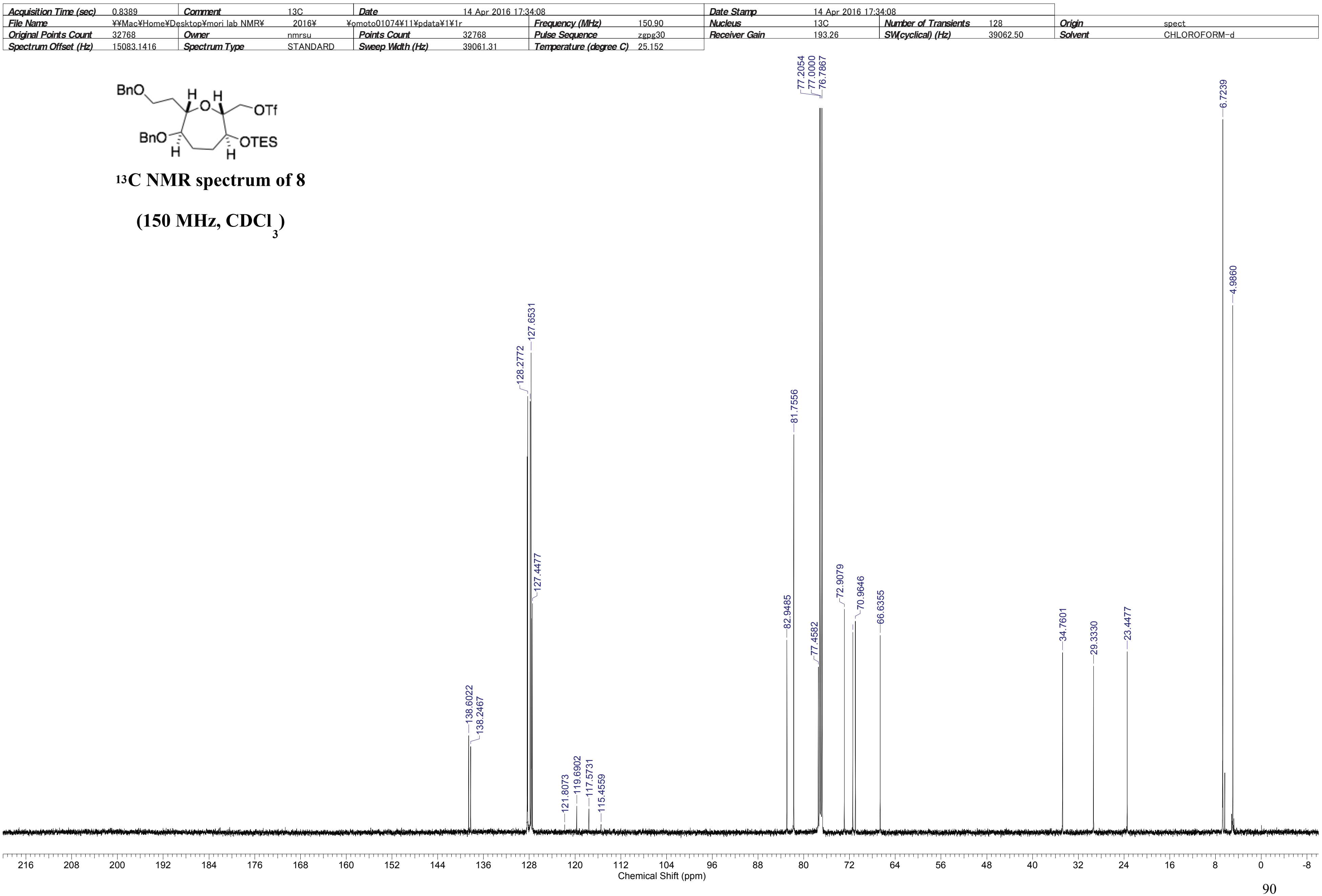


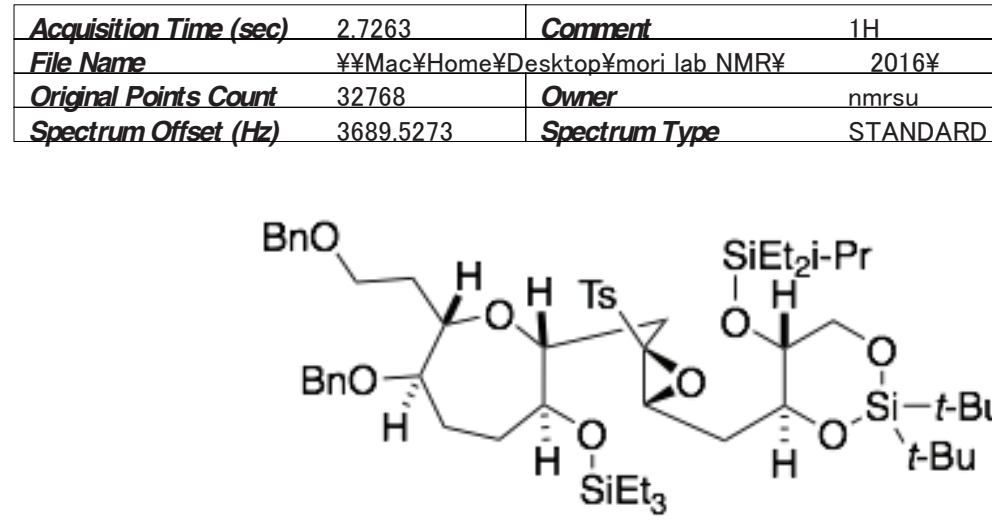

$(\alpha$-epoxide $: \beta$-epoxide $=88: 12$ )

\section{H NMR spectrum of 16}

(600 MHz, CDCl $\left.{ }_{3}\right)$

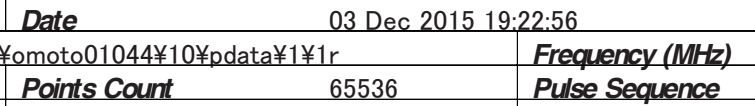

\begin{tabular}{lll} 
& & Date Stam \\
\hline Pulse Sequence & 600.13 & Nucleus \\
\hline
\end{tabular} Nucleus

03 Dec 2015 19:22:56

Number of Transients
SW(cyclical) $(\mathrm{Hz})$

12019.23

Origin
Solvent

spect
CHLOROFORM-d
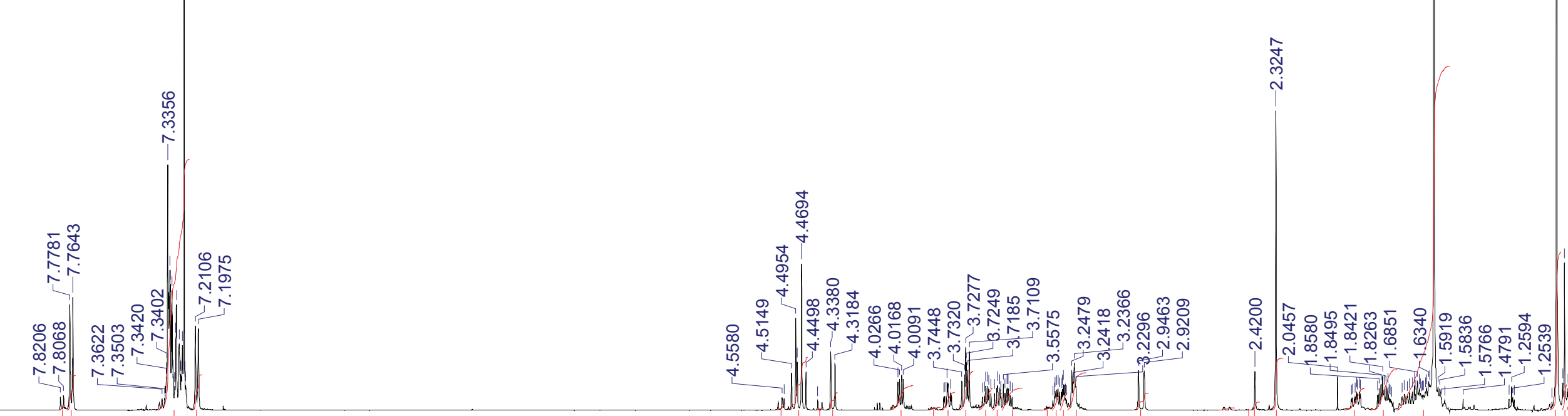
This report was created by ACD/NMR Processor Academic Edition. For more information go to www.acdlabs.com/nmrproc/

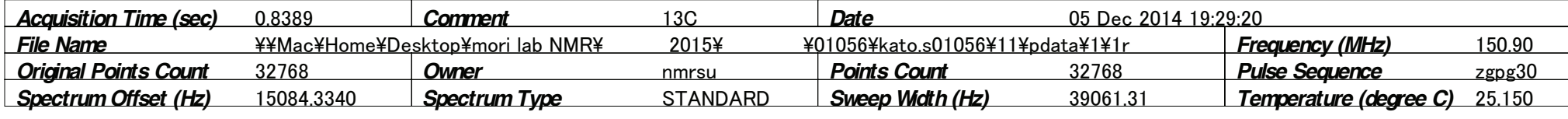

$$
\underbrace{\mathrm{S}_{\mathrm{SiEt}}}_{\hat{\mathrm{H}}}
$$

$(\alpha$-epoxide $: \beta$-epoxide $=88: 12)$

13C NMR spectrum of 16

\section{$\left(150 \mathrm{MHz}, \mathrm{CDCl}_{3}\right)$}

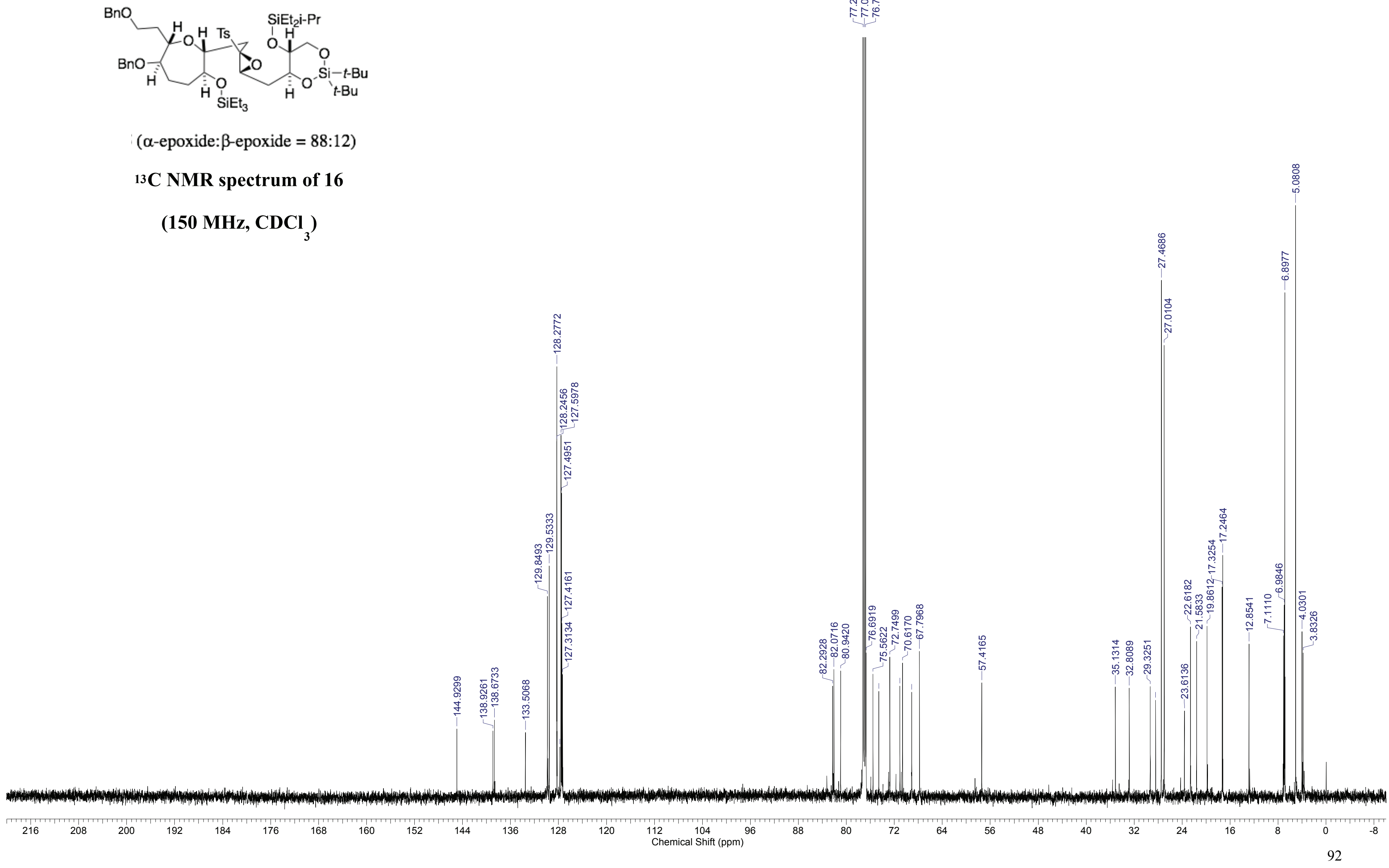

Date Stan
Nucleus Receiver Gain 05 Dec 2014 19:29:20

\section{Number of Transients
SWMcyclical) (Hz)} 328062.50 Origin
Solvent

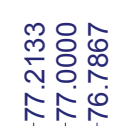


This report was created by ACD/NMR Processor Academic Edition. For more information go to www.acdlabs.com/nmrproc/

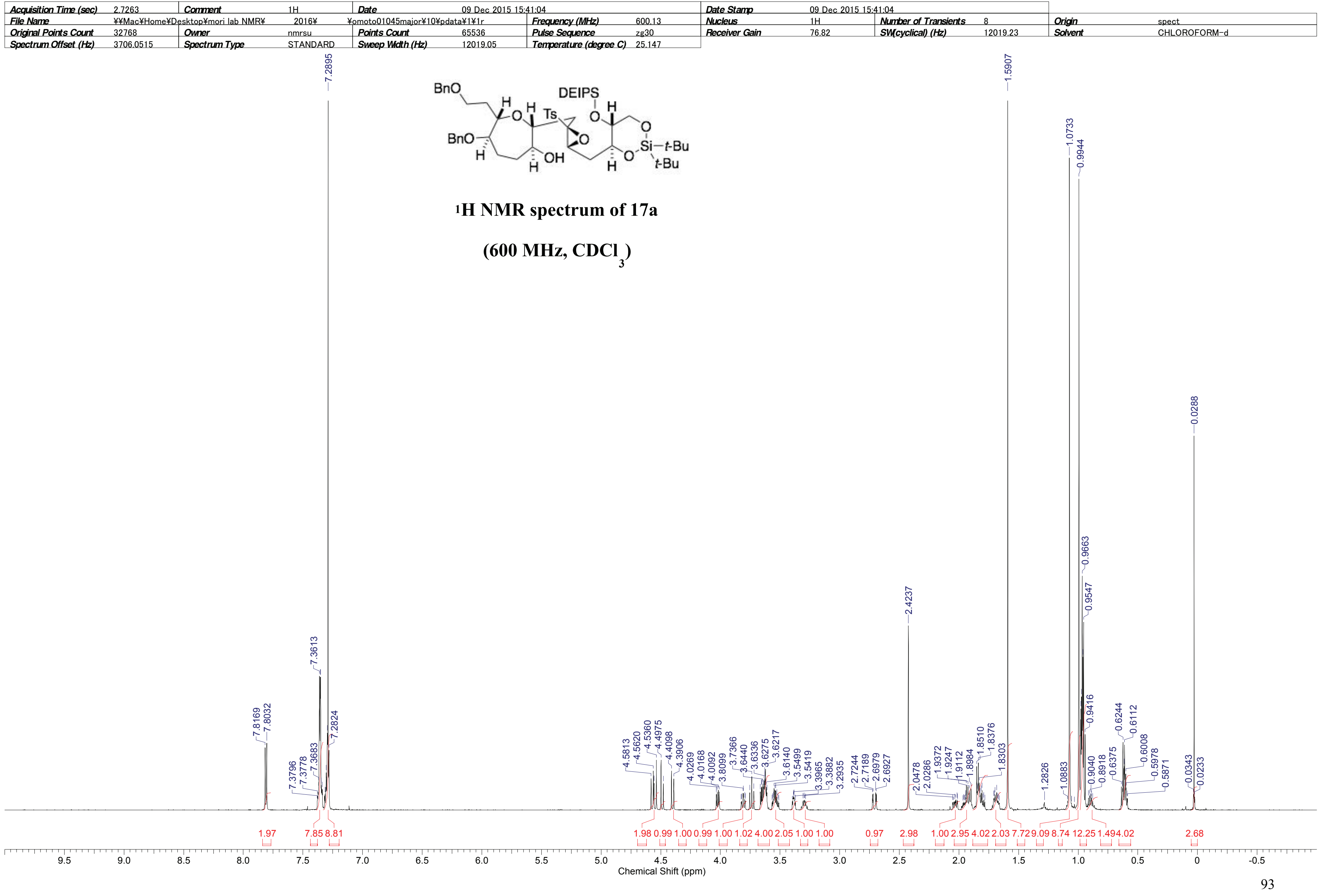



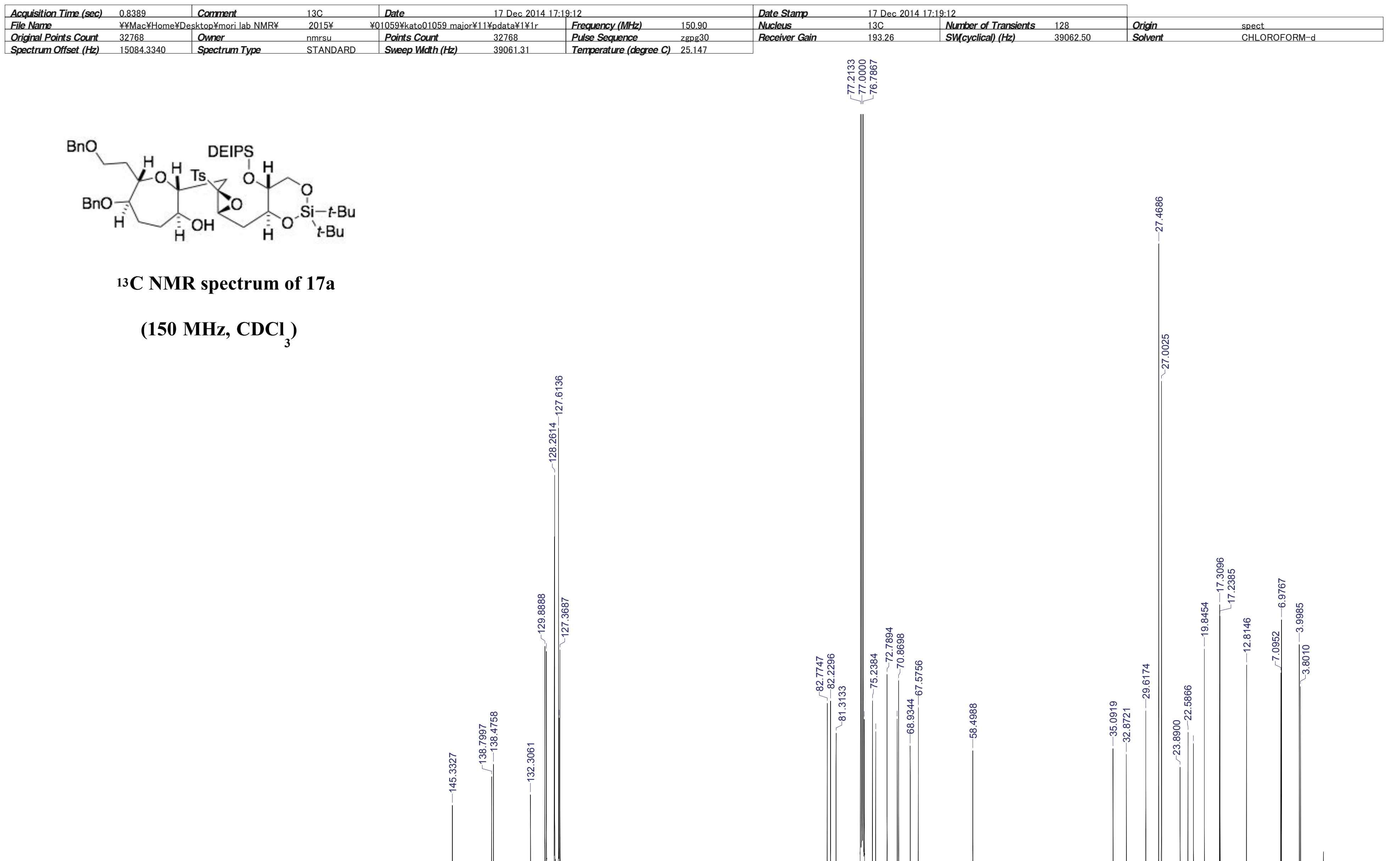

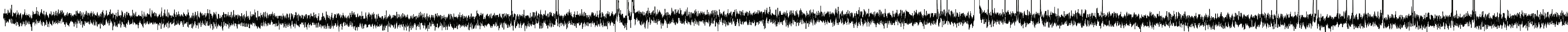


This report was created by ACD/NMR Processor Academic Edition. For more information go to www.acdlabs.com/nmrproc/

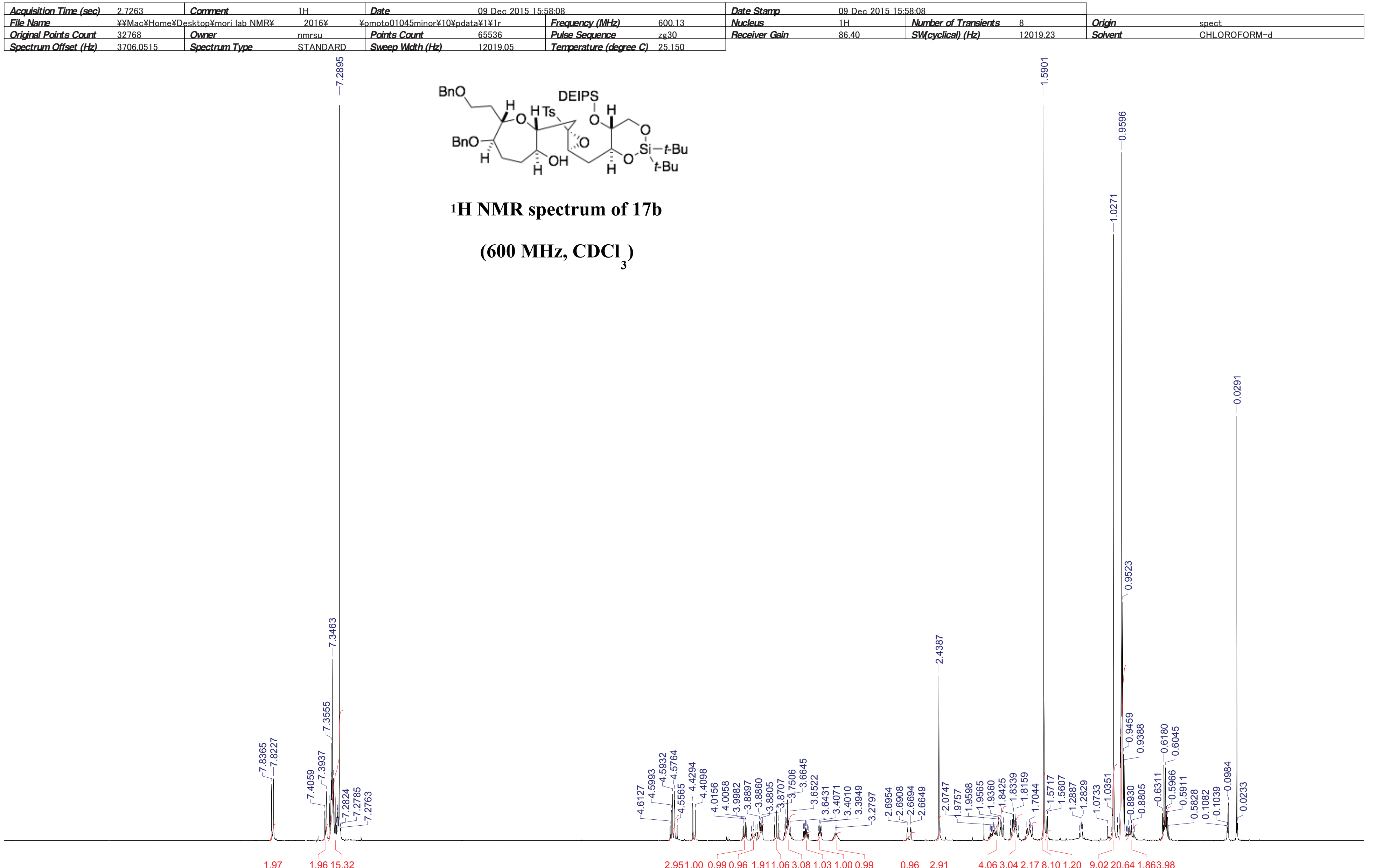

$2.951 .000 .990 .961 .911 .063 .081 .031 .000 .99 \quad 0.96 \quad 2.91$ 
This report was created by ACD/NMR Processor Academic Edition. For more information go to www.acdlabs.com/nmrproc/

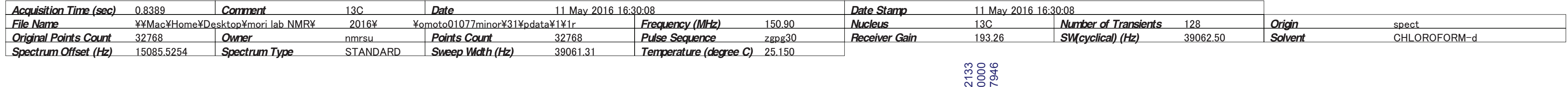

$$
\text { 每 }
$$

13C NMR spectrum of $17 \mathrm{~b}$

(150 MHz, CDCl $)$

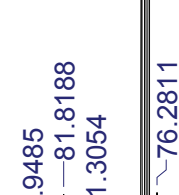

ז

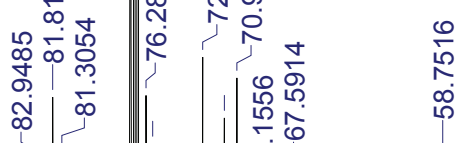

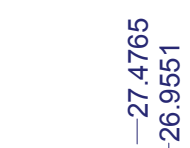

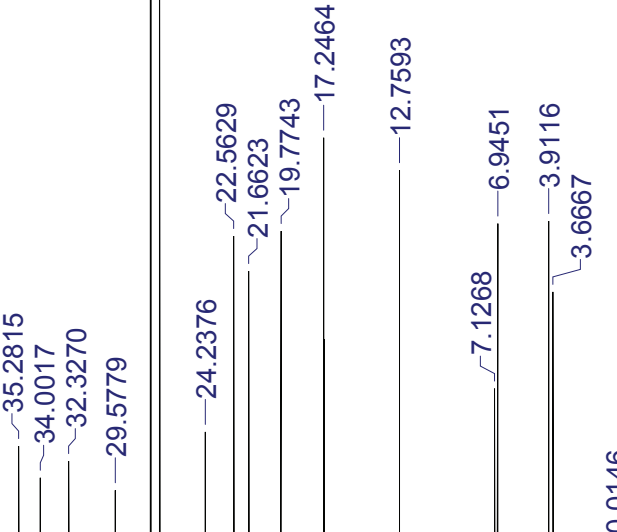

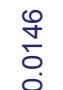

(1) 
This report was created by ACD/NMR Processor Academic Edition. For more information go to www.acdlabs.com/nmrproc/

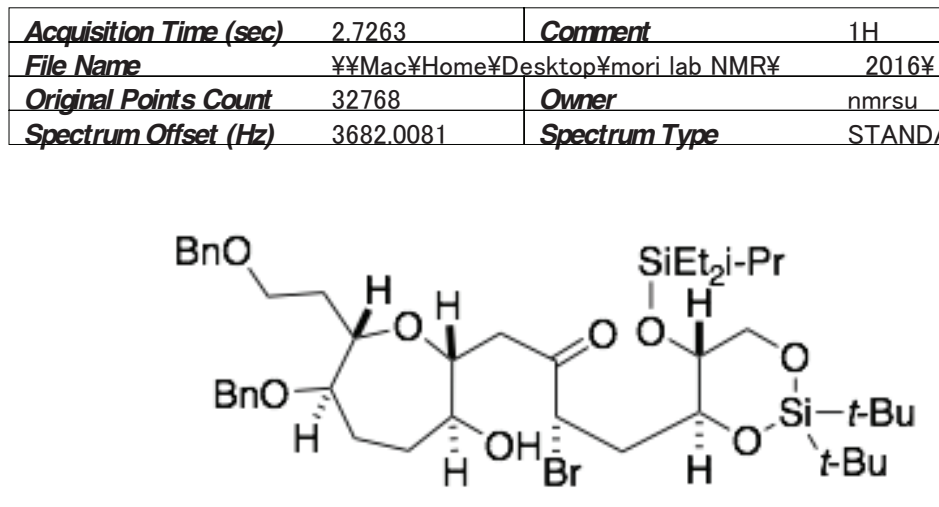

(70:15:15 tautomers)

1 H NMR spectrum of $6 \mathrm{a}$

(600 MHz, $\left.\mathrm{CDCl}_{3}\right)$

D16*
SANDARD

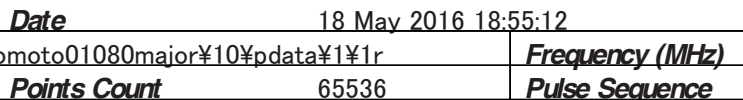

Frequency (MHz) $\quad 600.13$
Pulse Sequence
Fo30

Date Stamp
Nucleus

Nucleus
Receiver Gain

18 May 2016 18:55:

$\begin{array}{ll}\text { Number of Transients } & 8 \\ \text { SWWcyclical) (Hz) } & 12019.23\end{array}$

Origin
Solvent

怘

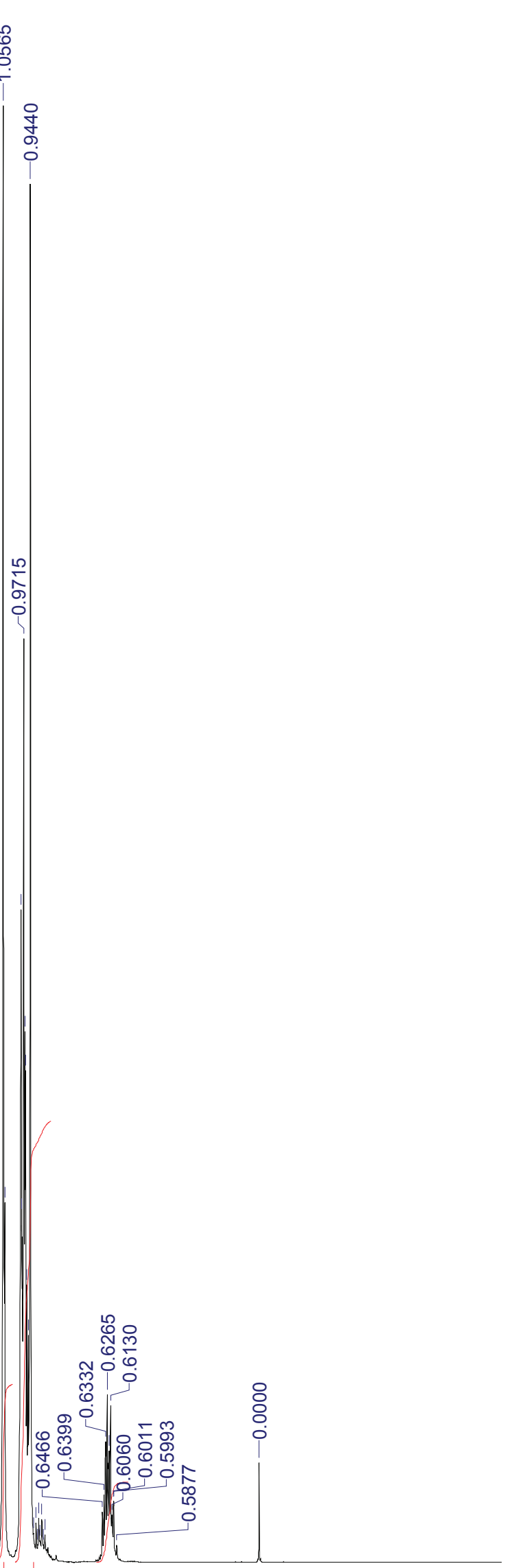

$0.710 .001 .042 .091 .410 .142 .370 .150 .151 .750 .763 .620 .910 .721 .410 .160 .140 .700 .160 .50 \quad 5.041 .382 .01$

9.370 .0323 .204 .22

11.03 
This report was created by ACD/NMR Processor Academic Edition. For more information go to www.acdlabs.com/nmrproc/

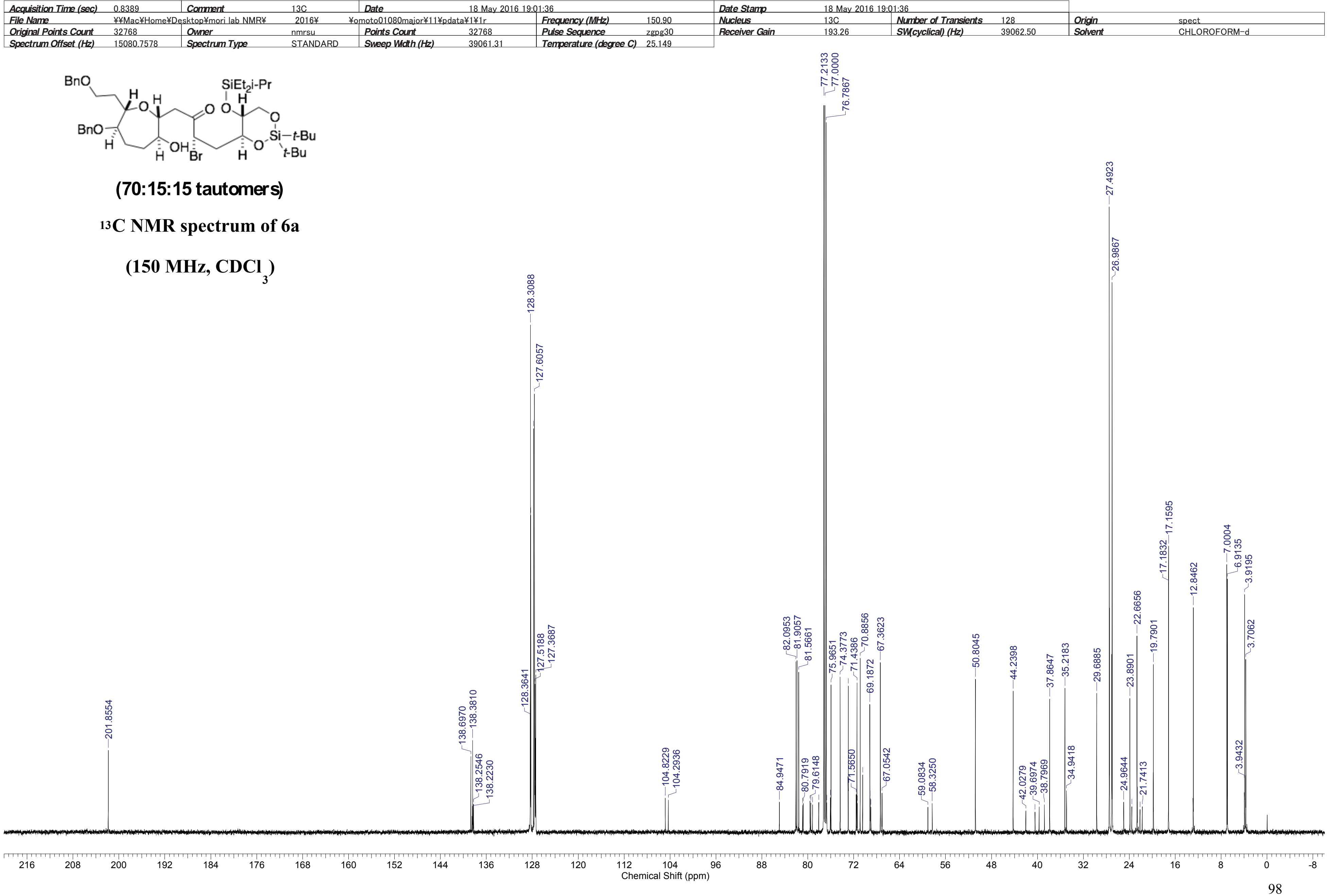


This report was created by ACD/NMR Processor Academic Edition. For more information go to www.acdlabs.com/nmrproc/

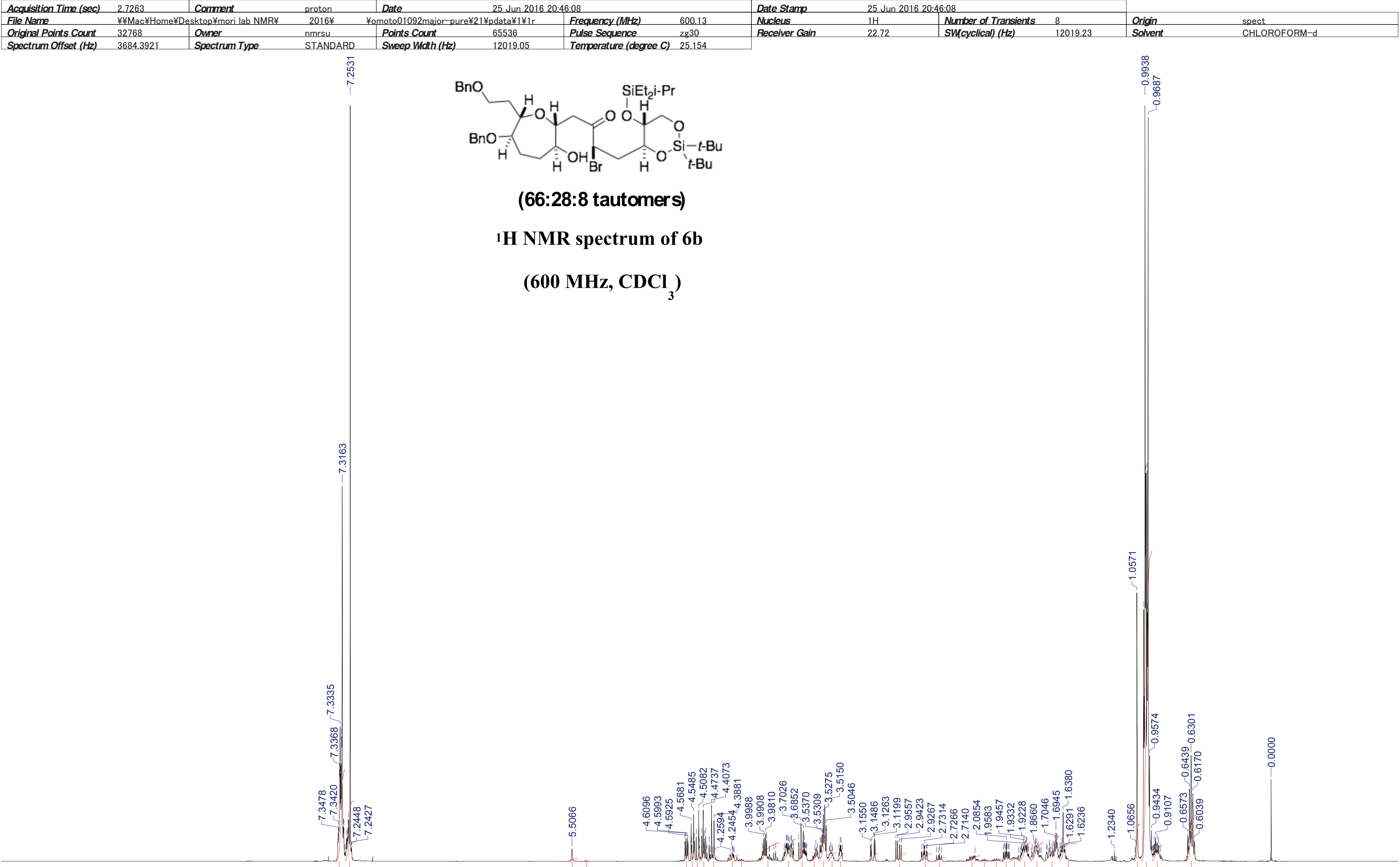


This report was created by ACD/NMR Processor Academic Edition. For more information go to www.acdlabs.com/nmrproc/

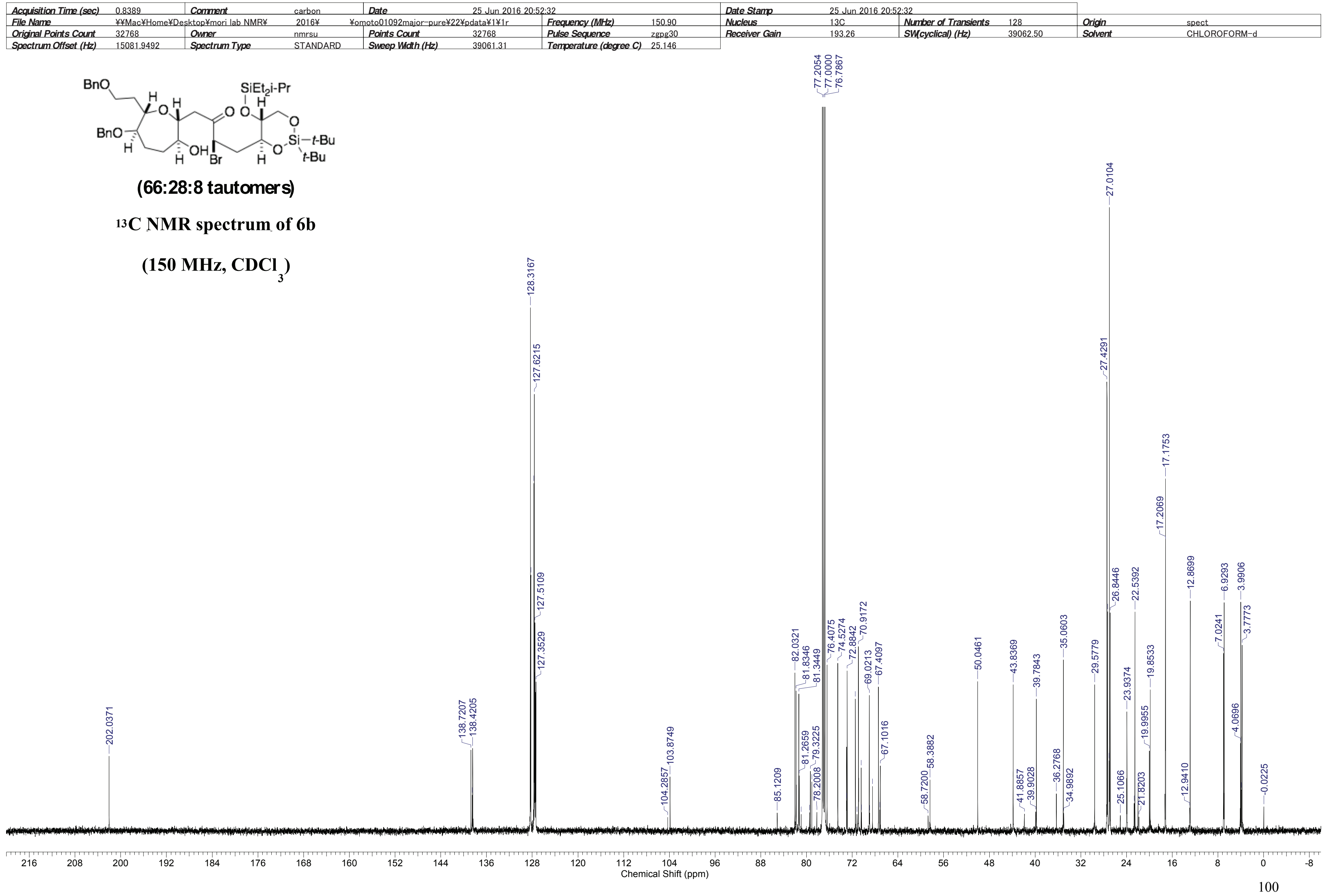


This report was created by ACD/NMR Processor Academic Edition. For more information go to www.acdlabs.com/nmrproc/

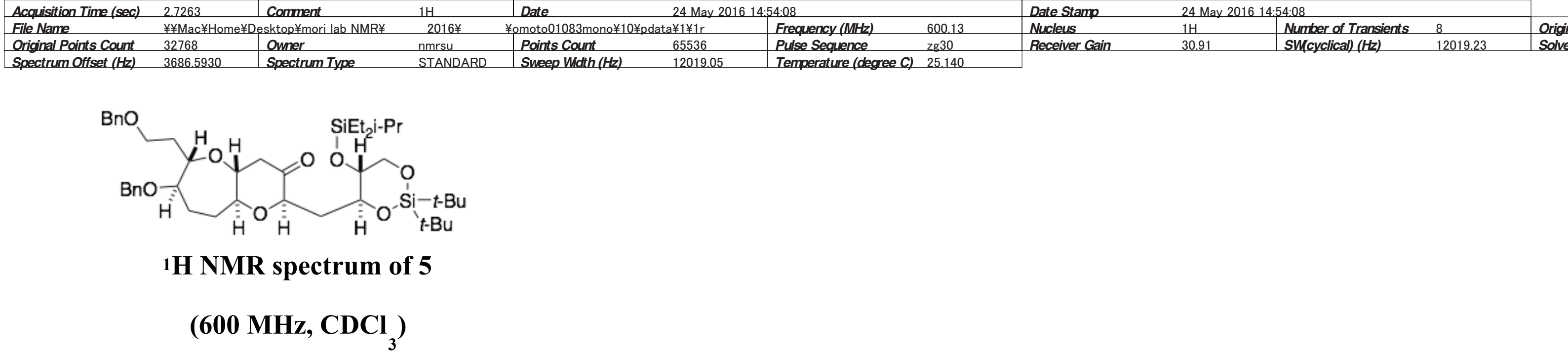

Origin
Solvent

离 spect

高
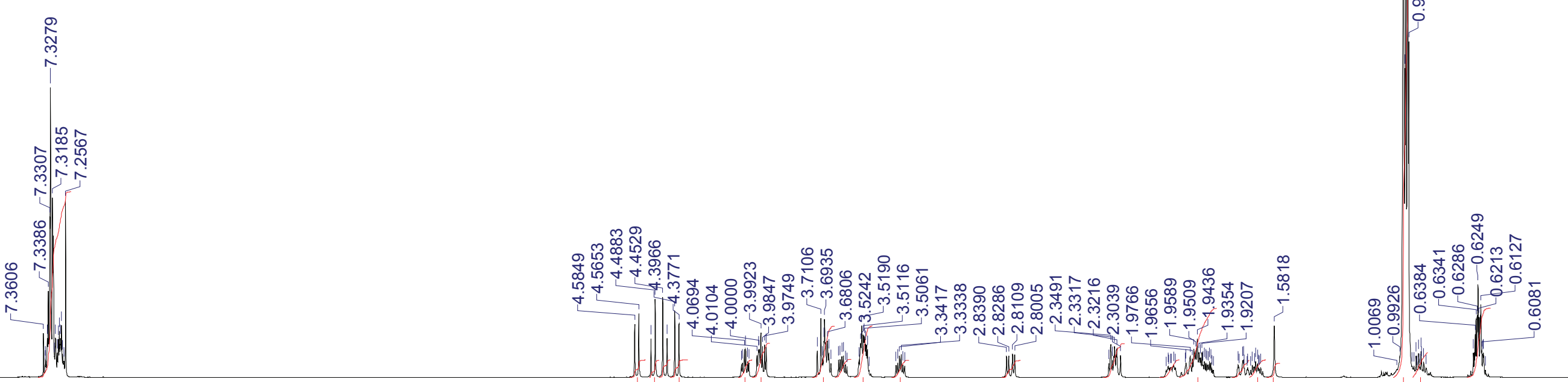

10.87

1.021 .011 .021 .031 .022 .013 .011 .073 .021 .00

0.99

$1.99 \quad 1.034 .051 .011 .020 .84$

30.041 .464 .07 
This report was created by ACD/NMR Processor Academic Edition. For more information go to www.acdlabs.com/nmrproc/

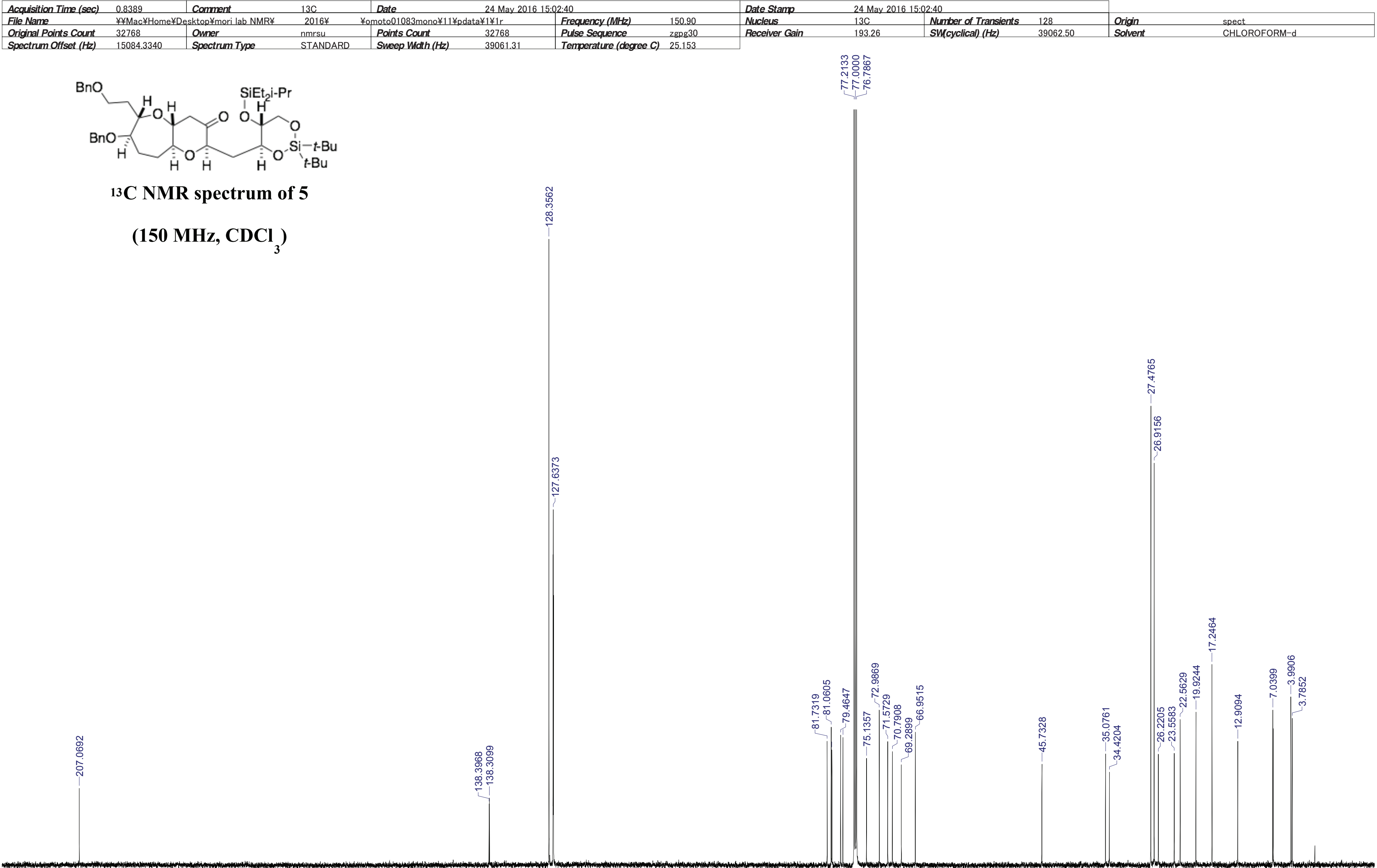

13C NMR spectrum of 5

(150 $\left.\mathrm{MHz}_{2} \mathrm{CDCl}_{3}\right)$ 
This report was created by ACD/NMR Processor Academic Edition. For more information go to www.acdlabs.com/nmrproc/

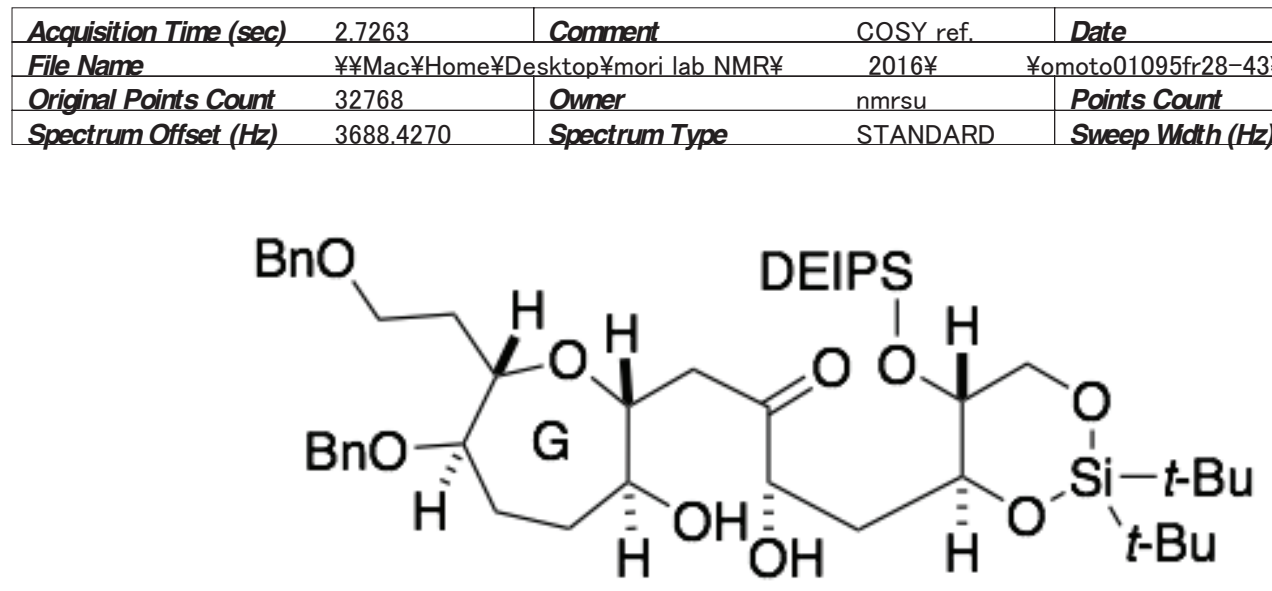

(67:19:14 tautomers)

1H NMR spectrum of S3

(600 MHz, CDCl )

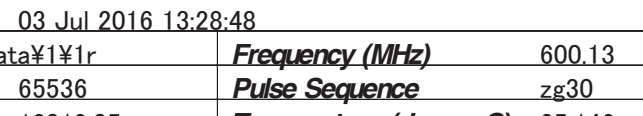

Date Stamp
Nucleus Nucleus 03 Jul 2016 13:28:48 $\begin{array}{ll}\text { Number of Transients } & 16 \\ \text { SWlaclical) (Hz) } & 1201923\end{array}$ \begin{tabular}{l|l}
\hline 16 & Origin \\
\hline 12019.23 & Solvent \\
\hline
\end{tabular}

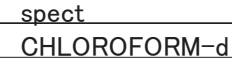


This report was created by ACD/NMR Processor Academic Edition. For more information go to www.acdlabs.com/nmrproc/

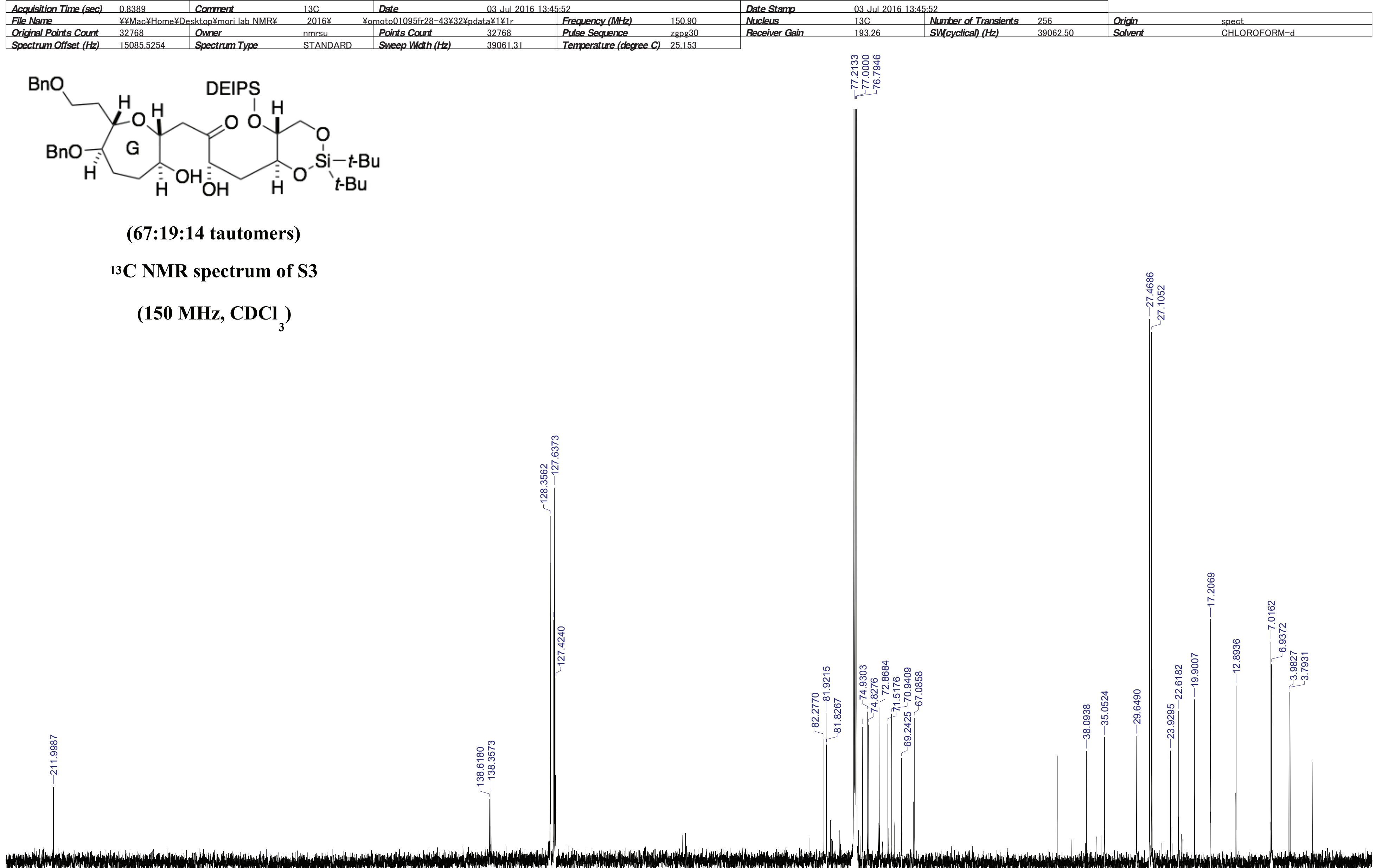

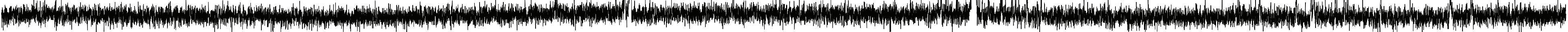

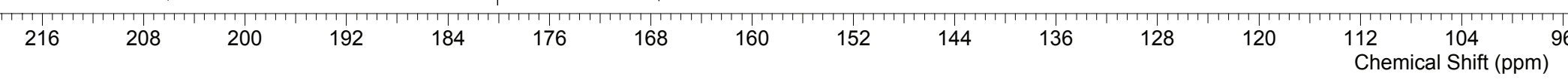


This report was created by ACD/NMR Processor Academic Edition. For more information go to www.acdlabs.com/nmrproc/

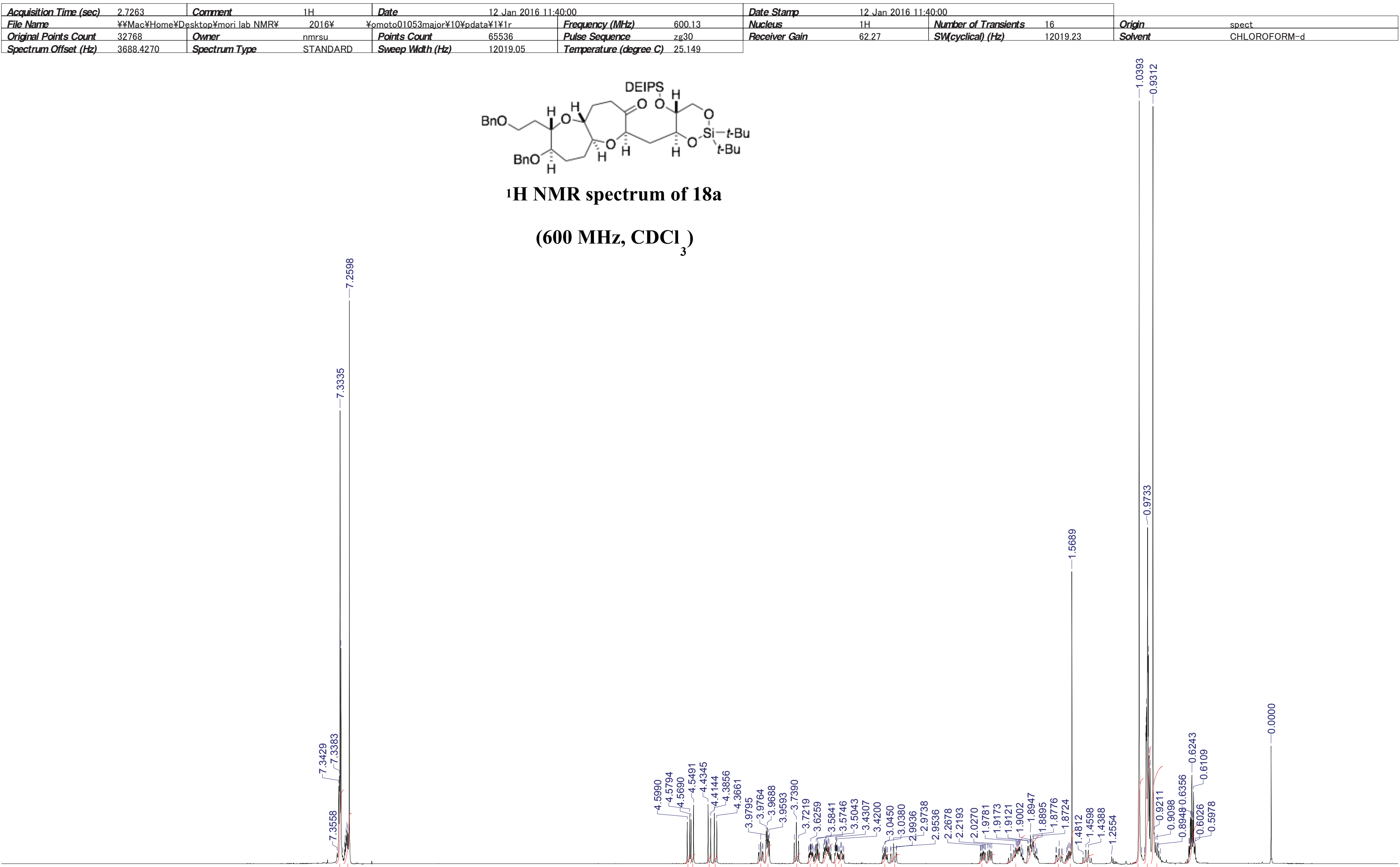

8.035 .46

1.01 1.02 1.01 1.00 1.04 2.01 1.06 1.031.05 2.121.00 1.041.001.00 $\quad 0.980 .993 .053 .071 .023 .610 .989 .2612 .7610 .554 .22$ 
This report was created by ACD/NMR Processor Academic Edition. For more information go to www.acdlabs.com/nmrproc/

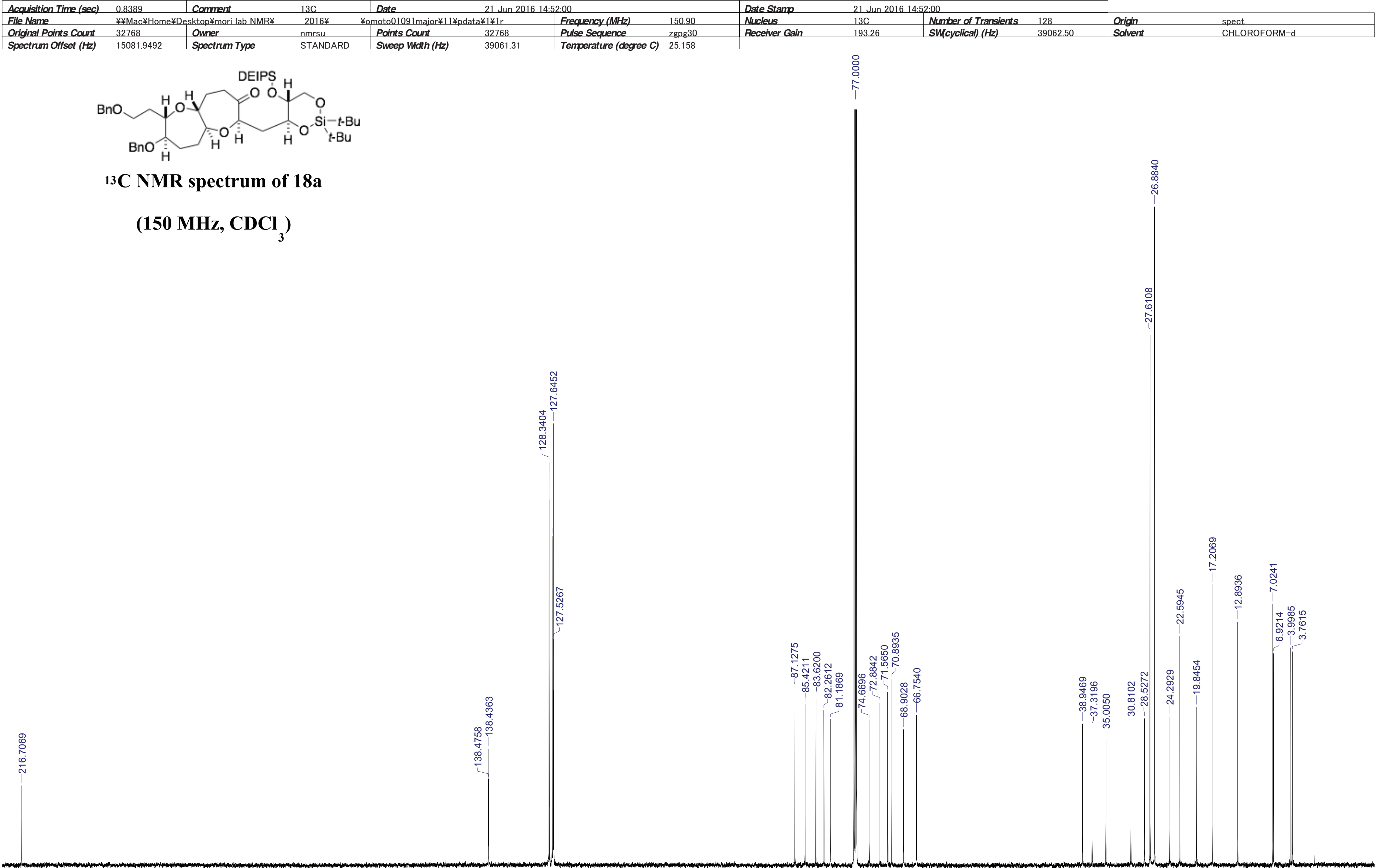


This report was created by ACD/NMR Processor Academic Edition. For more information go to www.acdlabs.com/nmrproc/

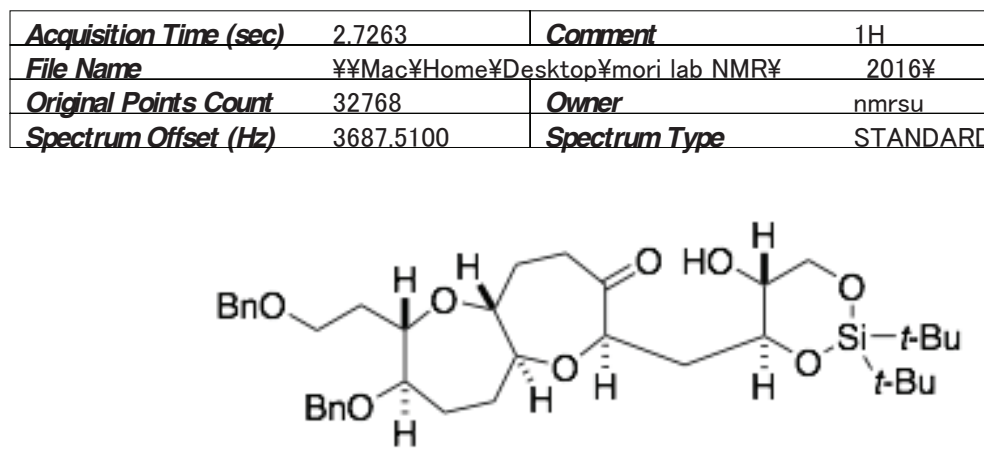

(84:16 tautomers)

$1 \mathrm{H}$ NMR spectrum of $18 \mathrm{~b}$

(600 MHz, CDCl $\left.{ }_{3}\right)$

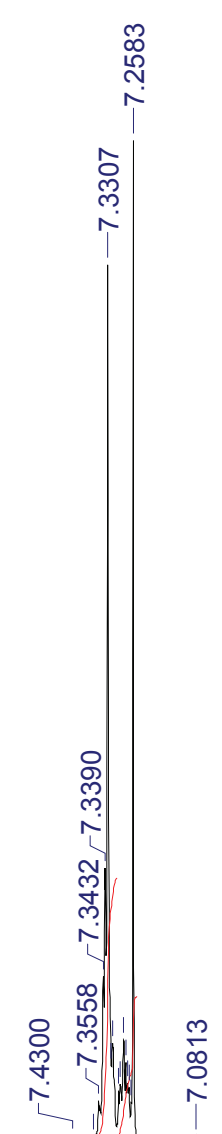


This report was created by ACD/NMR Processor Academic Edition. For more information go to www.acdlabs.com/nmrproc/

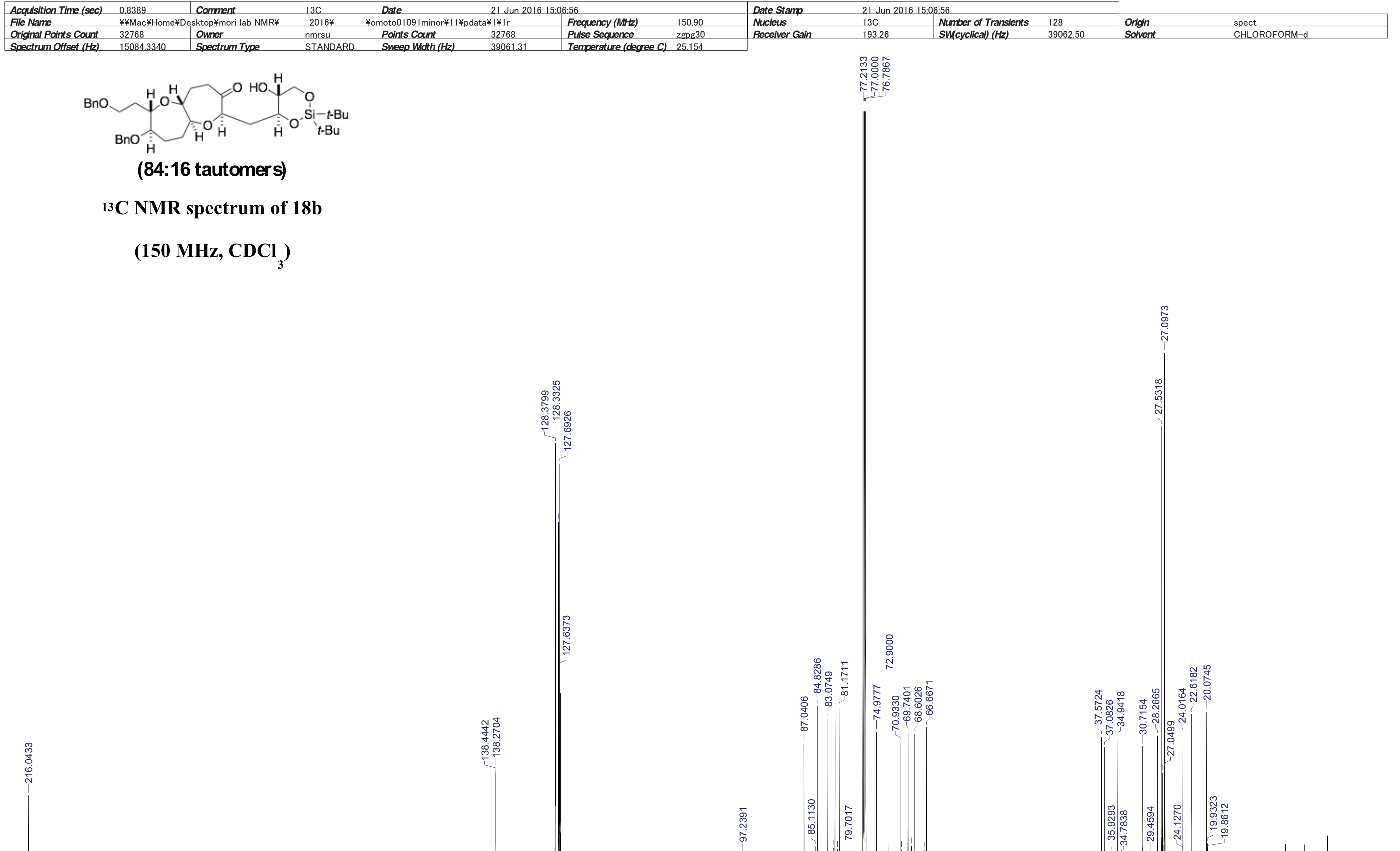

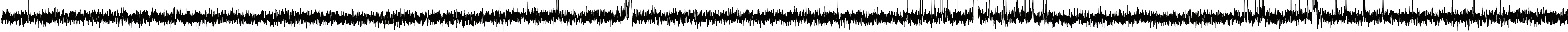


This report was created by ACD/NMR Processor Academic Edition. For more information go to www.acdlabs.com/nmrproc/

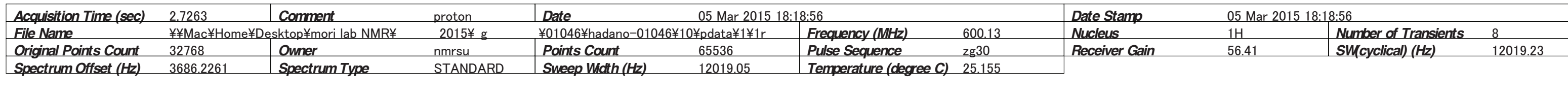

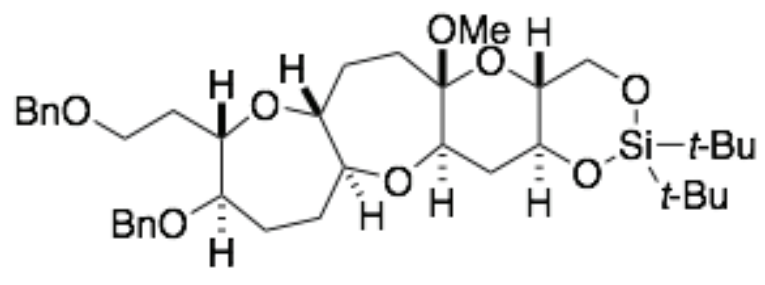

1H NMR spectrum of 19

(600 MHz, CDCl )

8.192 .93

1.021.001.01 0.991 .022 .020 .001 .992 .171 .063 .013 .01

$0.976 .051 .052 .083 .33 \quad 9.059 .27$ 
This report was created by ACD/NMR Processor Academic Edition. For more information go to www.acdlabs.com/nmrproc/

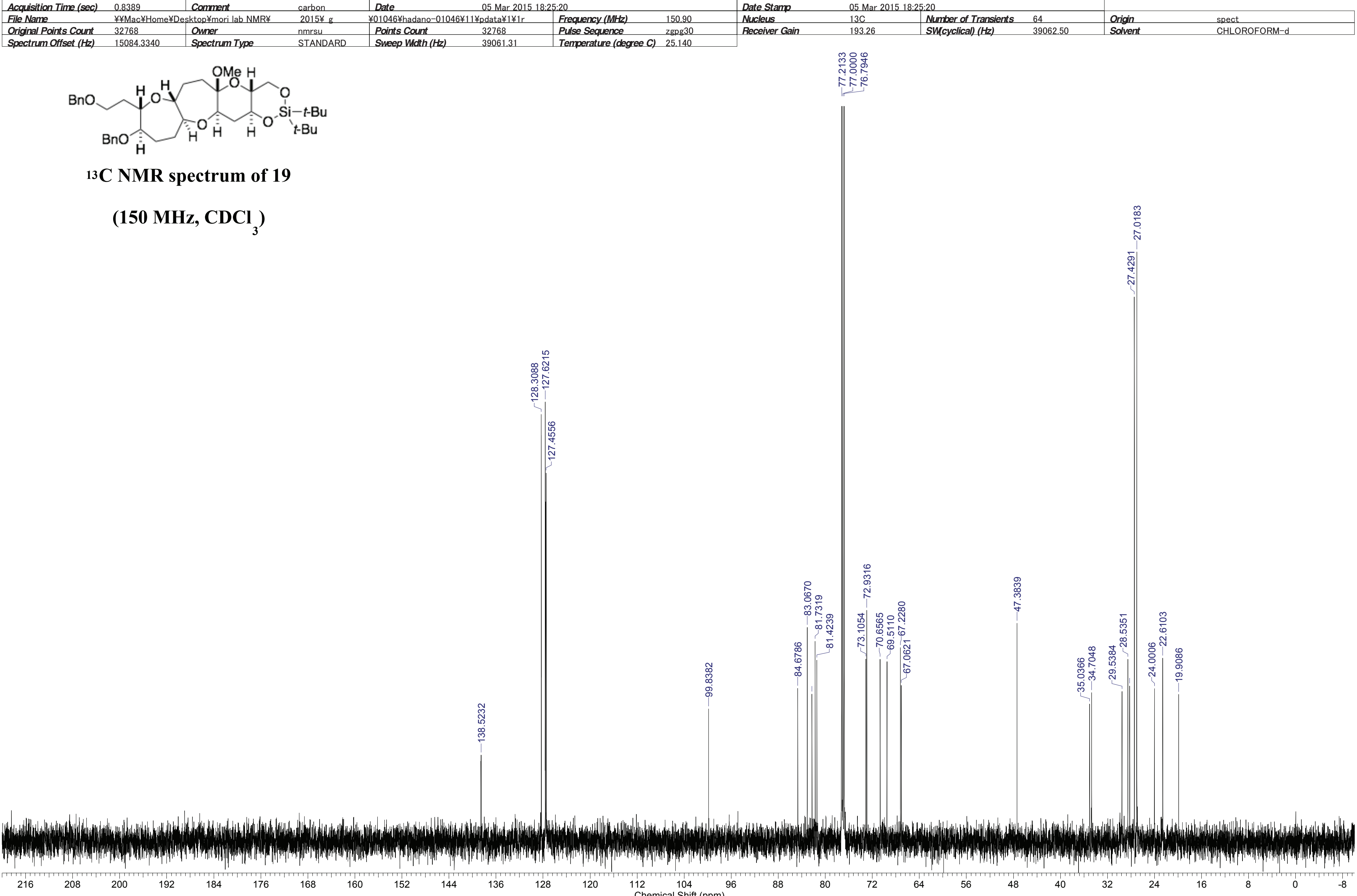


This report was created by ACD/NMR Processor Academic Edition. For more information go to www.acdlabs.com/nmrproc/

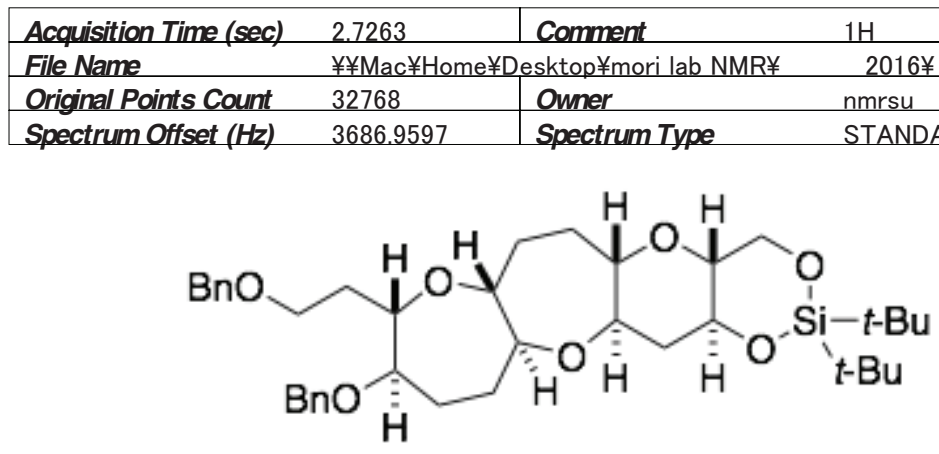

Date Stam

30 Aug 2016 13:33:04

Number of Transients 8

Frequency (MHz)
Pulse Sequence

Rucleus

\begin{tabular}{l|l}
11 & Number of Transients \\
\hline 56.41 & SW(cyclical) (Hz)
\end{tabular}

\begin{tabular}{l|l}
\hline 8 & Origin \\
\hline 12019.23 & Solvent \\
\hline
\end{tabular}

Sigin

2019/04/27 20:02:15

1H NMR spectrum of 20

(600 MHz, CDCl )

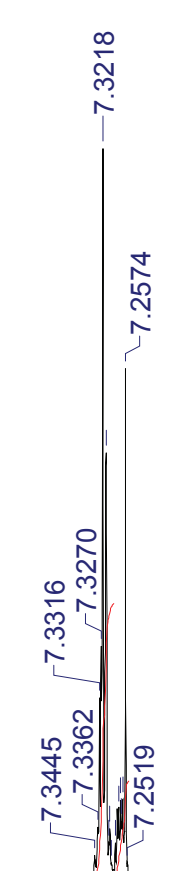

7.822 .82

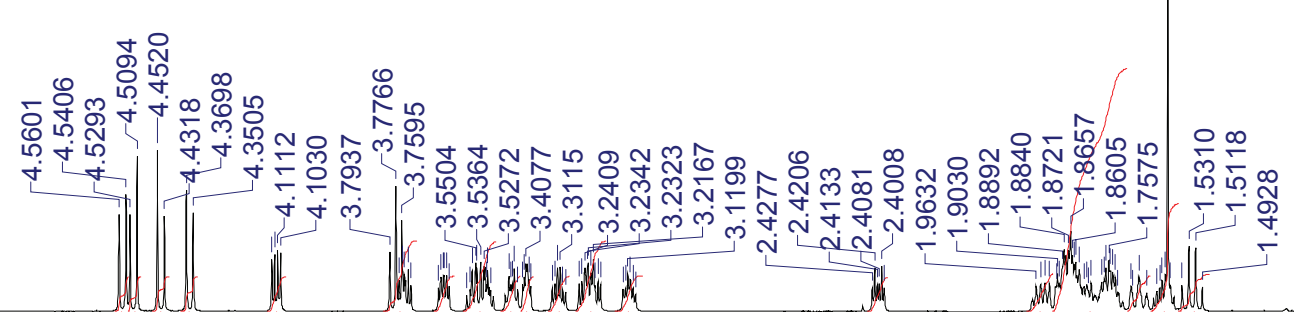

$0.970 .970 .980 .970 .961 .980 .981 .960 .990 .970 .981 .971 .00 \quad 0.95$

$1.066 .831 .003 .031 .04 \quad 8.809 .07$

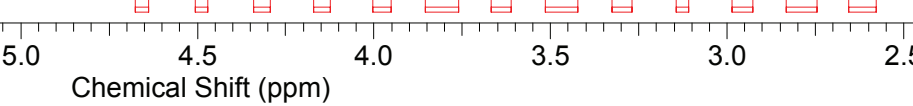


This report was created by ACD/NMR Processor Academic Edition. For more information go to www.acdlabs.com/nmrproc/

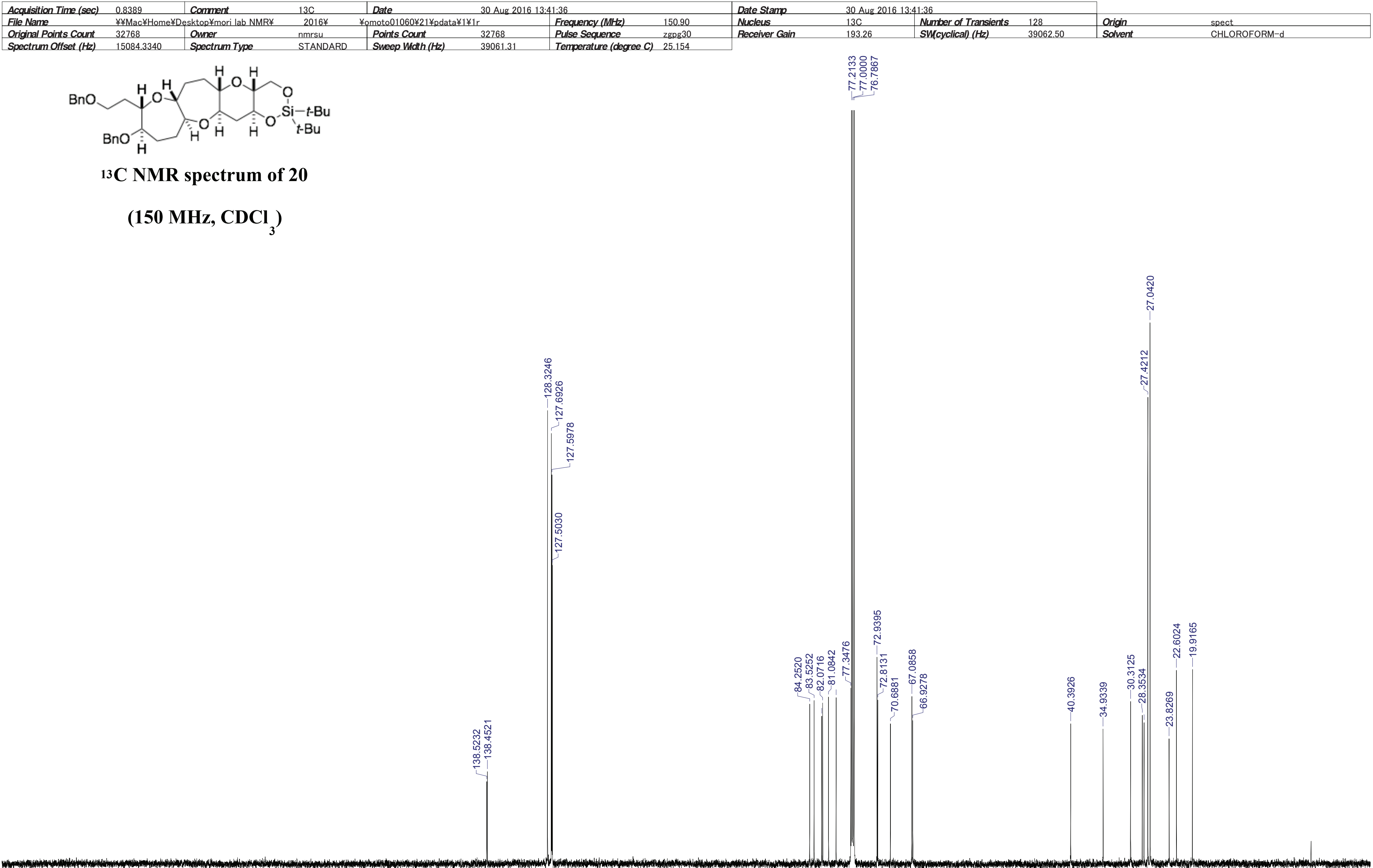


This report was created by ACD/NMR Processor Academic Edition. For more information go to www.acdlabs.com/nmrproc/

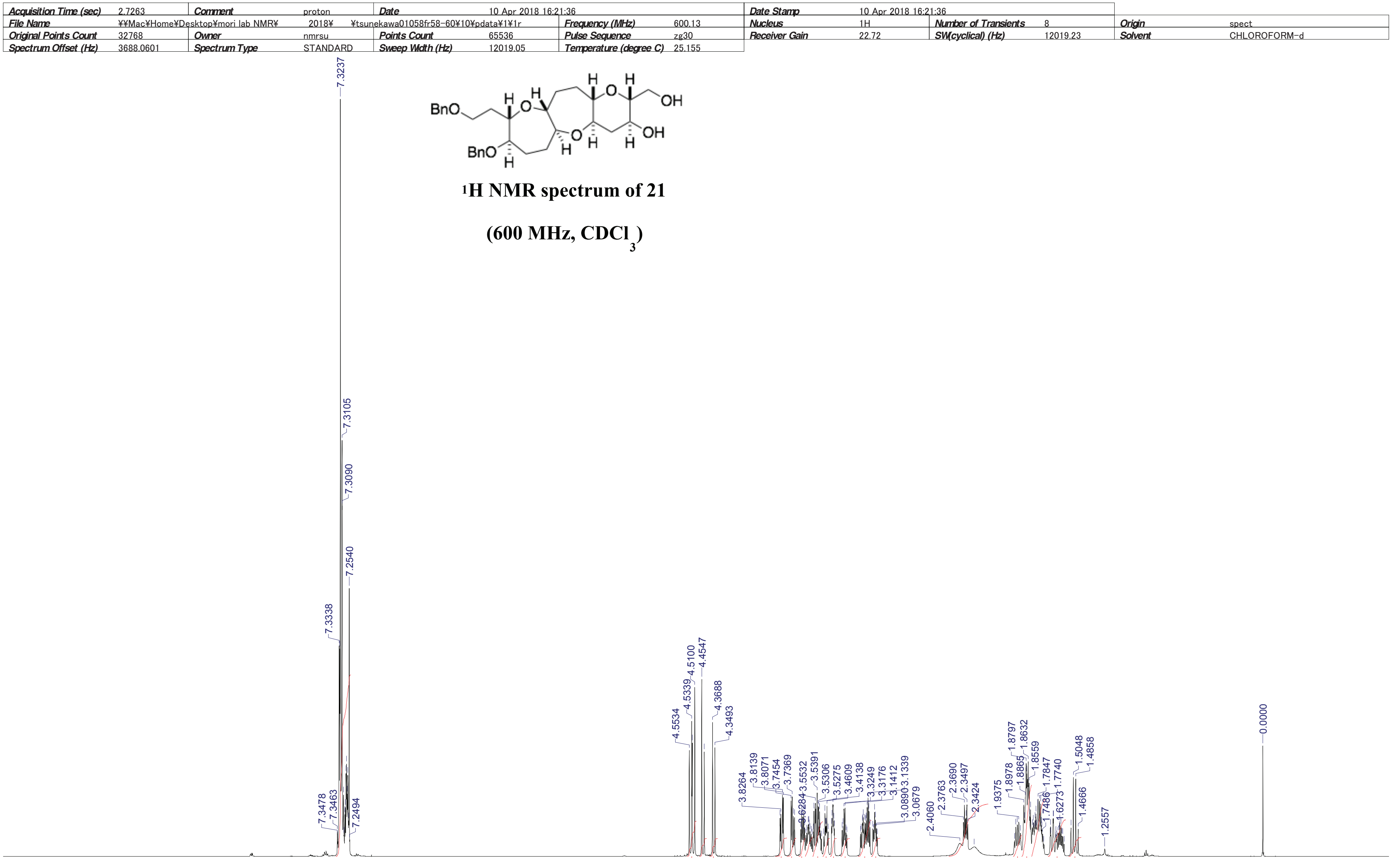

10.17

$1.981 .011 .001 .031 .021 .021 .111 .941 .011 .011 .002 .021 .01 \quad 2.94 \quad 1.204 .003 .202 .051 .06$

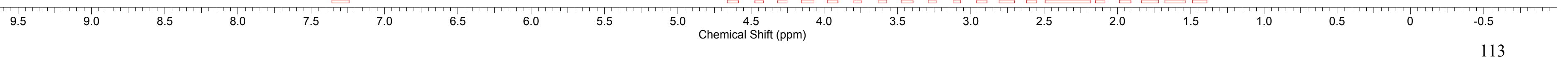


This report was created by ACD/NMR Processor Academic Edition. For more information go to www.acdlabs.com/nmrproc/

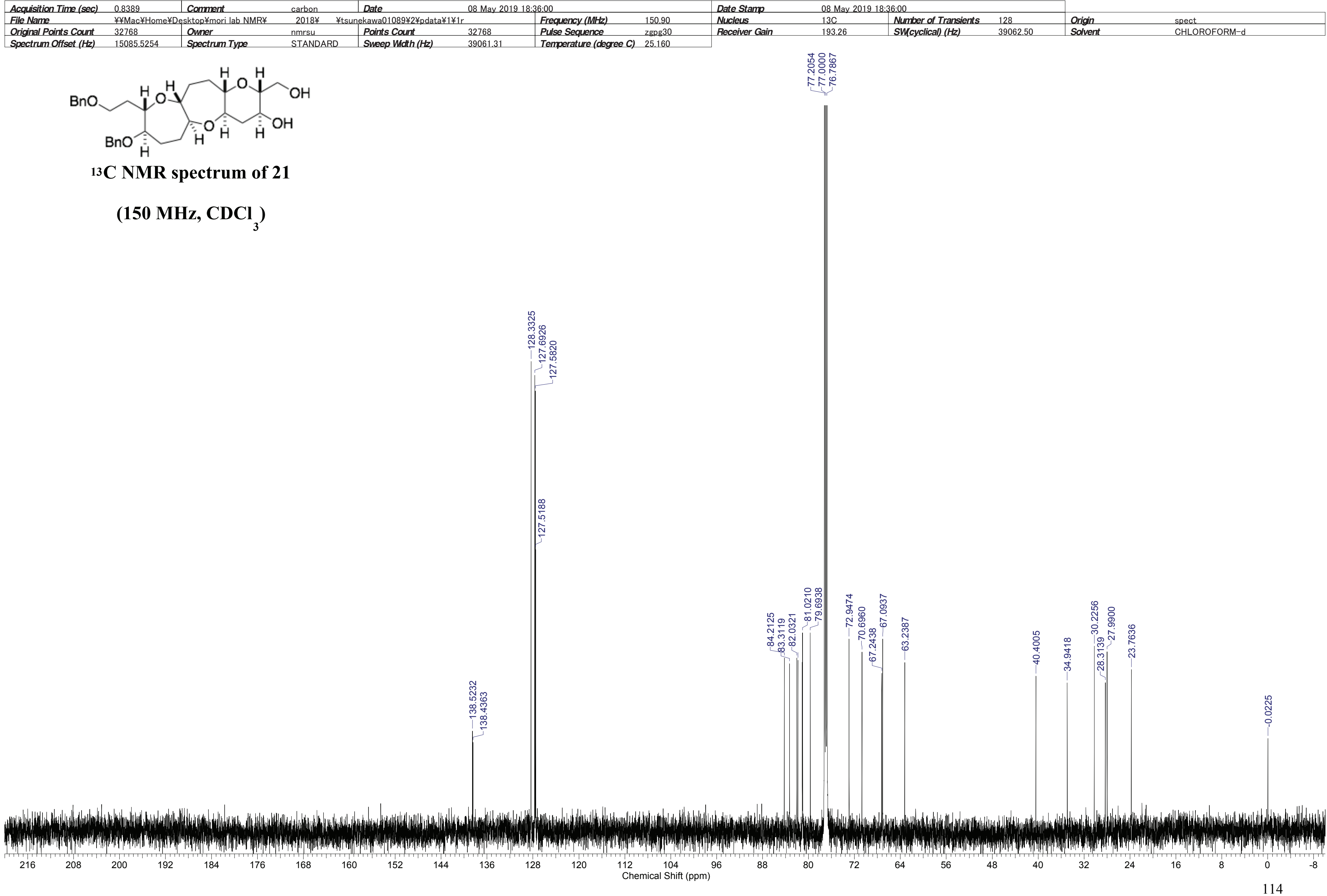


This report was created by ACD/NMR Processor Academic Edition. For more information go to www.acdlabs.com/nmrproc/

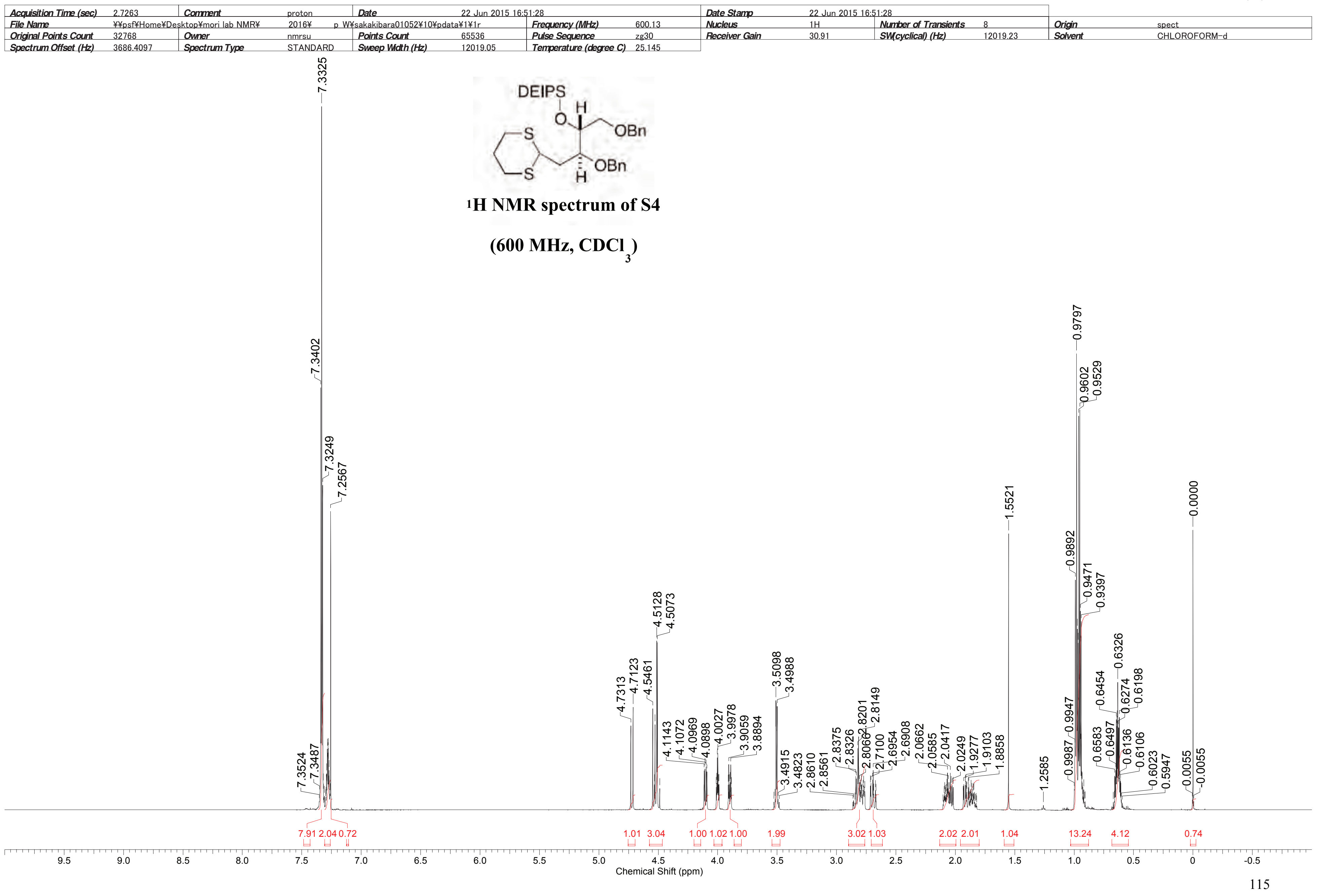


This report was created by ACD/NMR Processor Academic Edition. For more information go to www.acdlabs.com/nmrproc/

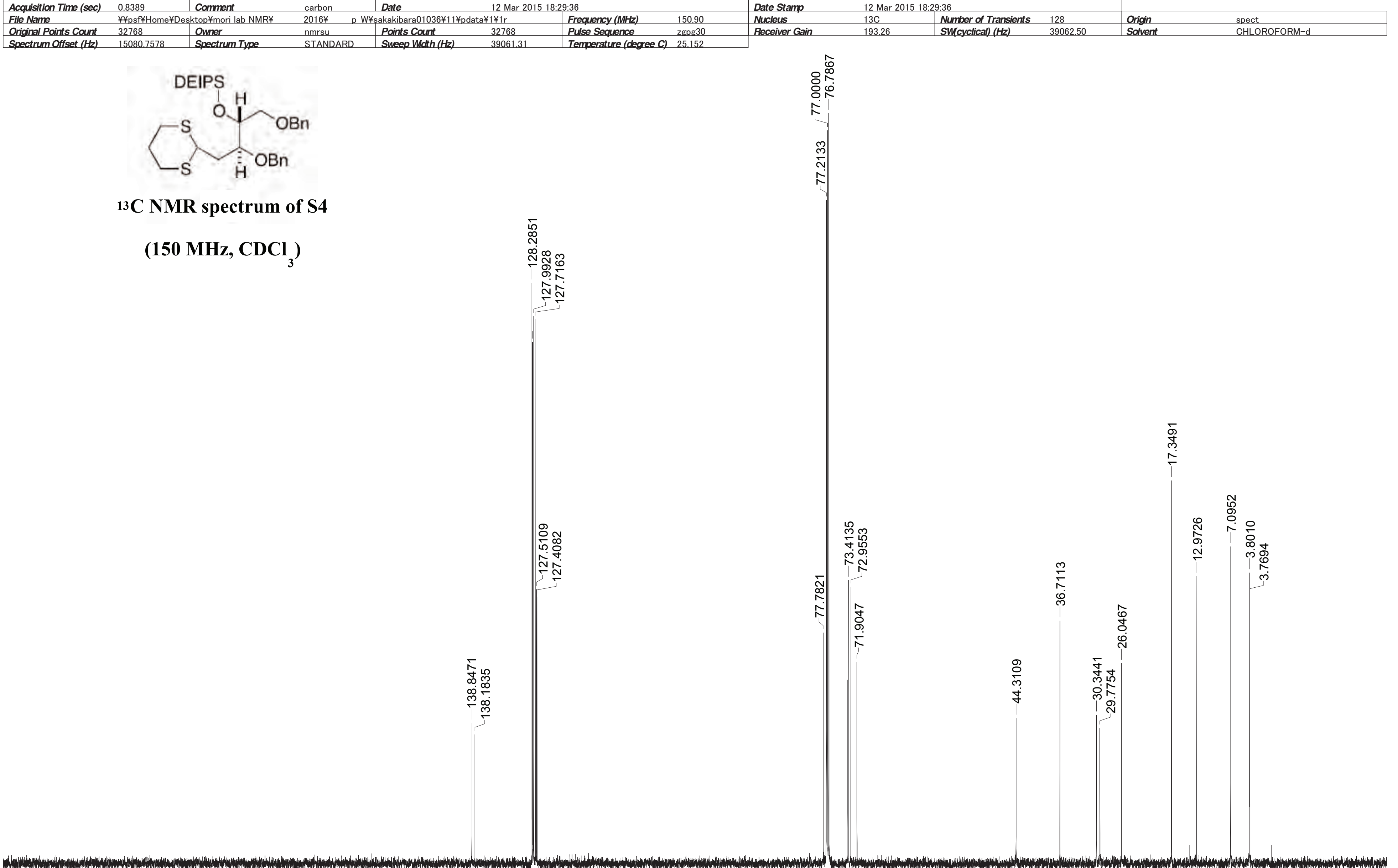


This report was created by ACD/NMR Processor Academic Edition. For more information go to www.acdlabs.com/nmrproc/

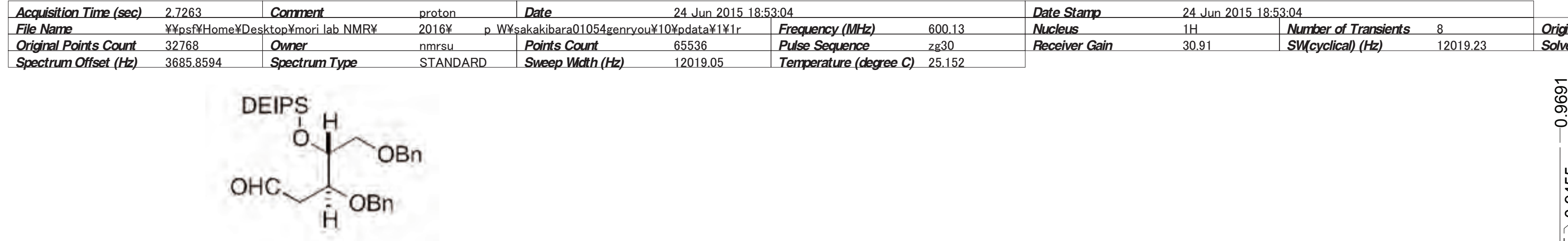

1H NMR spectrum of S5

$\left(600 \mathrm{MHz}, \mathrm{CDCl}_{3}\right)$
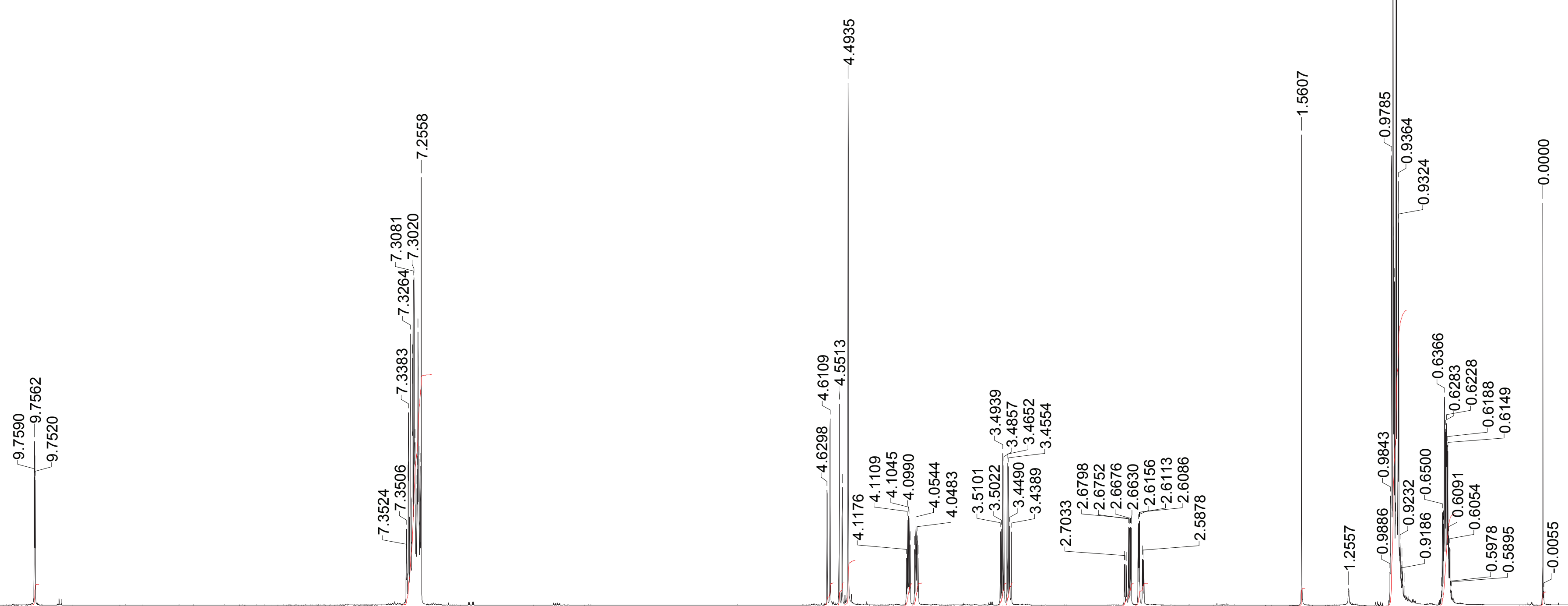

0.94

10.47

1.011 .012 .020 .971 .00

0.991 .01

0.971 .00

0.76

$13.39 \quad 4.16$

0.59

$\begin{array}{lllllllllll}9.5 & 9.0 & 8.5 & 8.0 & 7.5 & 7.0 & 6.5 & 6.0 & 5.5 & 5.0 & 4.5 \\ \text { Chemical Shift (ppm) }\end{array}$

4.5 
This report was created by ACD/NMR Processor Academic Edition. For more information go to www.acdlabs.com/nmrproc/

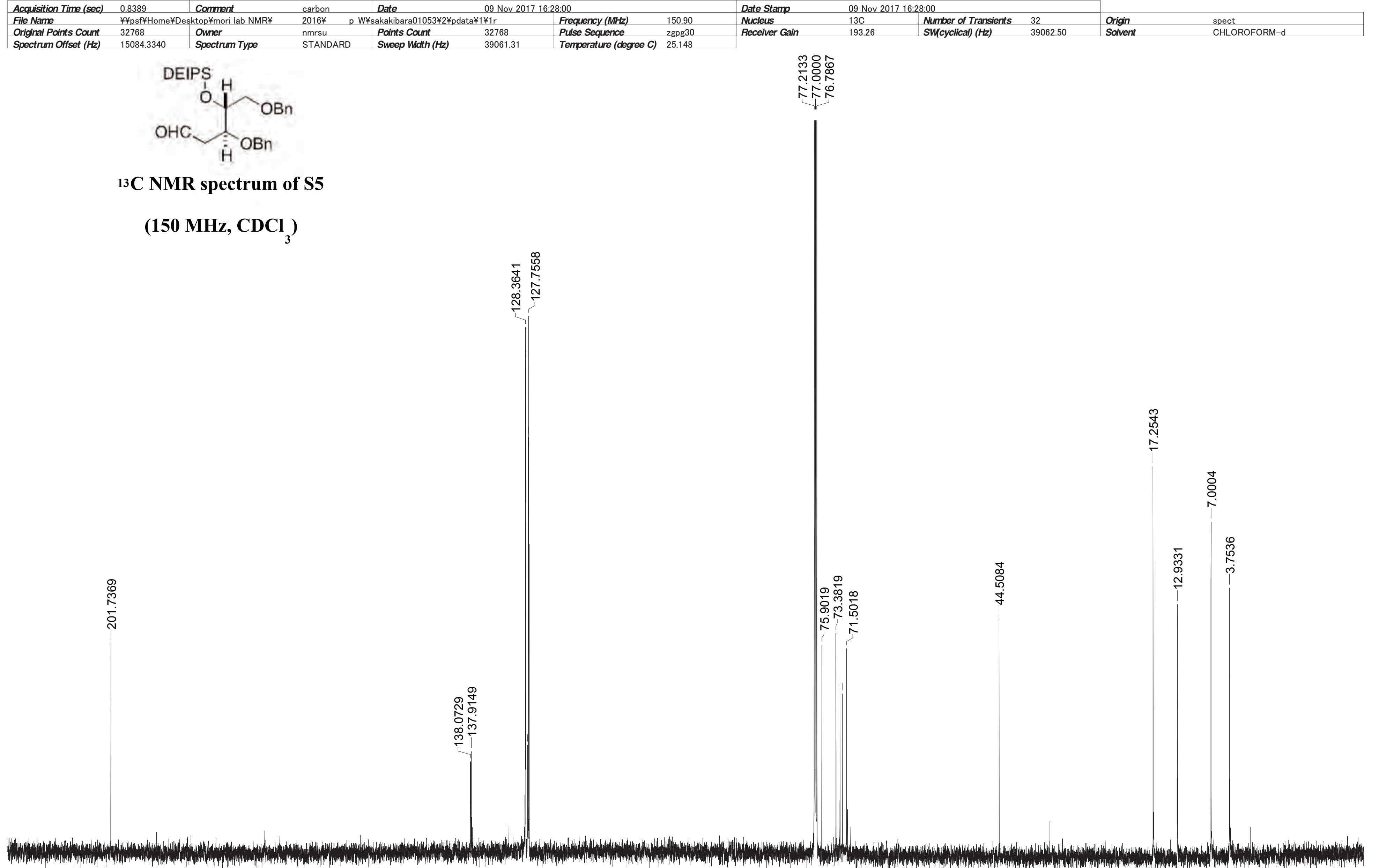

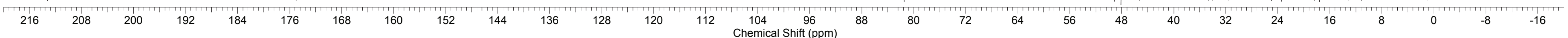


This report was created by ACD/NMR Processor Academic Edition. For more information go to www.acdlabs.com/nmrproc/
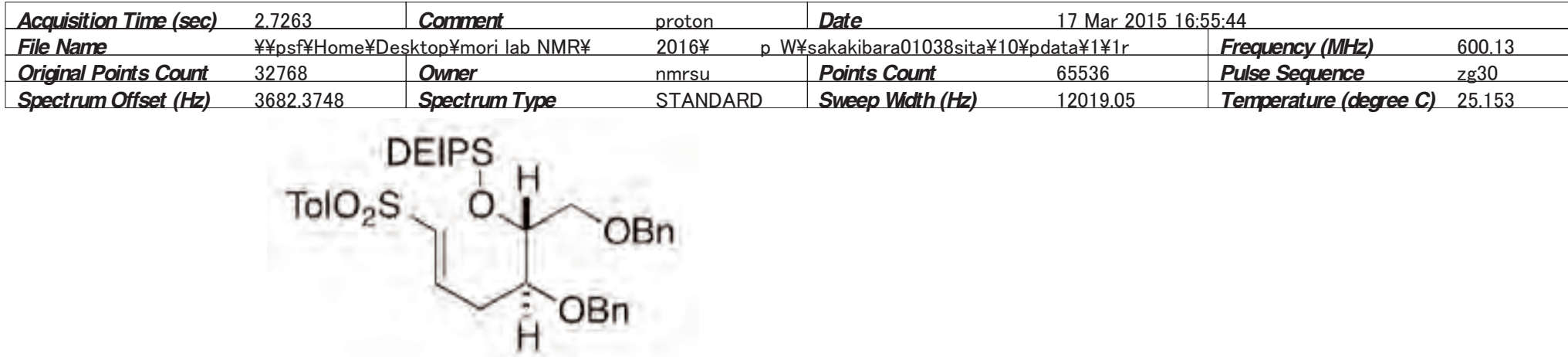

1 H NMR spectrum of S6

$\left(600 \mathrm{MHz}, \mathrm{CDCl}_{3}\right)$
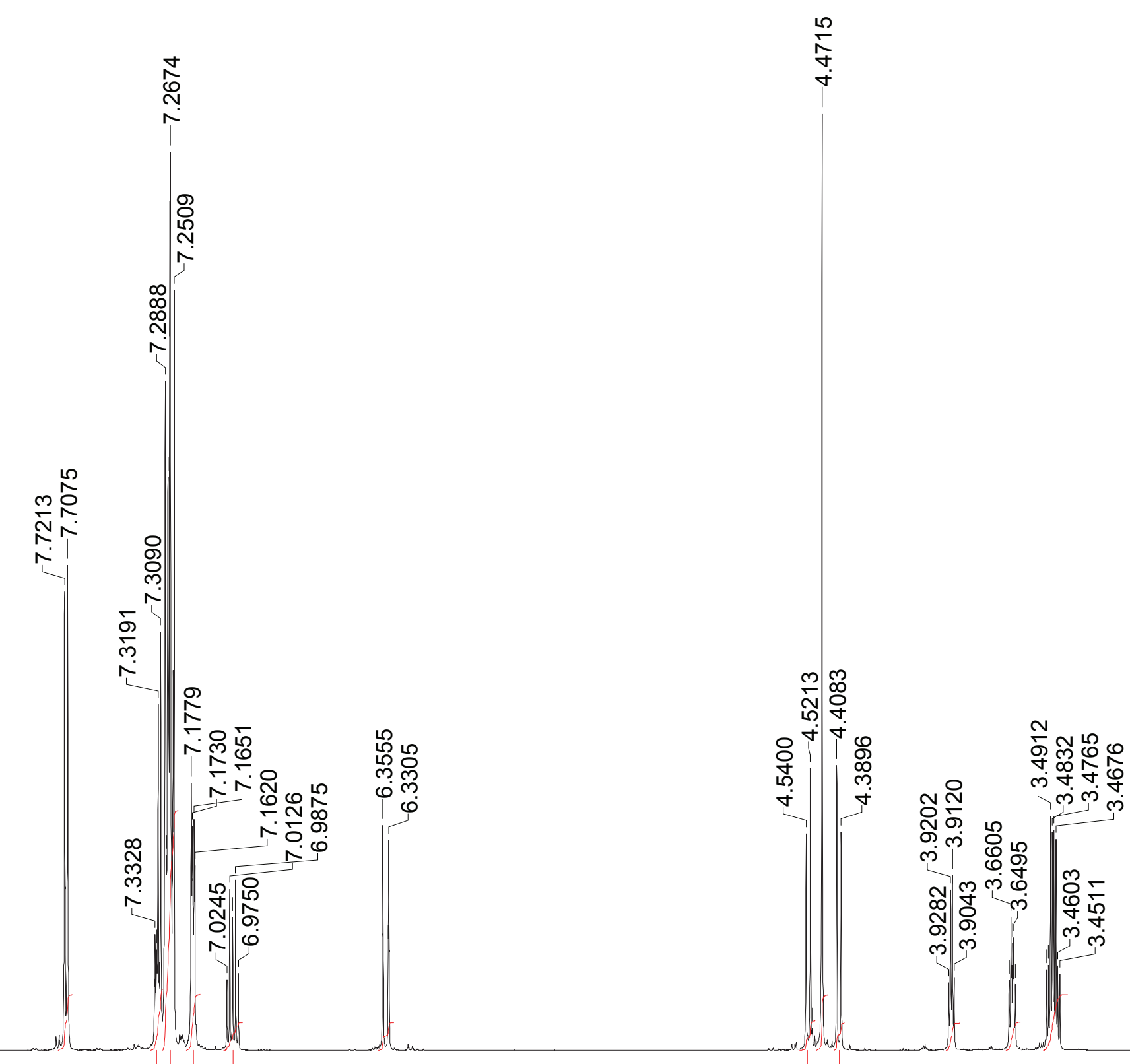

1.00

Date Stamp

Nucleus
Receiver Gain

2.012 .228 .702 .031 .01

1.082 .010 .99

$0.99 \quad 1.022 .02$ 17 Mar
$1 \mathrm{H}$
30.91 Number of Transients
SW(cyclical) (Hz)

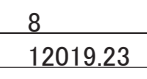

orgin

㤻

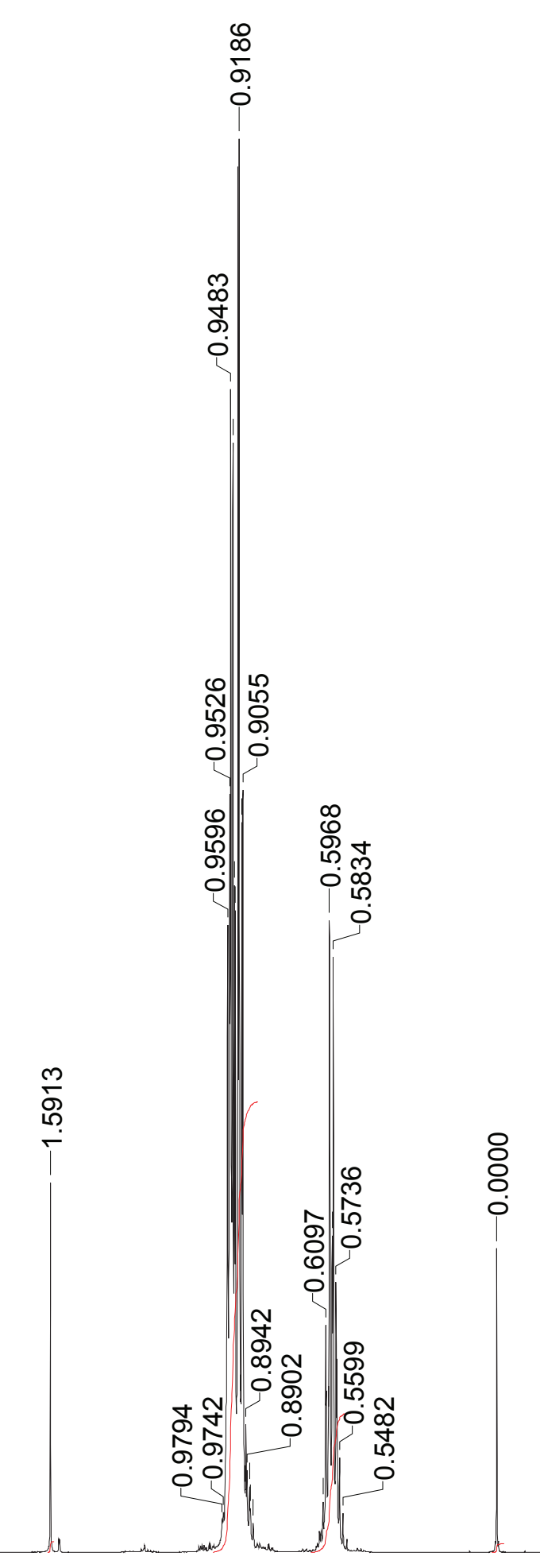

Chemical Shift (ppm)

0.32

$13.57 \quad 4.20$ 0.25

$\begin{array}{llllllllll}9.5 & 9.0 & 8.5 & 8.0 & 7.5 & 7.0 & 6.5 & 6.0 & 5.5 & 5.0 \\ & & & & & & & & & \\ \text { Chemical Shift (ppm) }\end{array}$


This report was created by ACD/NMR Processor Academic Edition. For more information go to www.acdlabs.com/nmrproc/

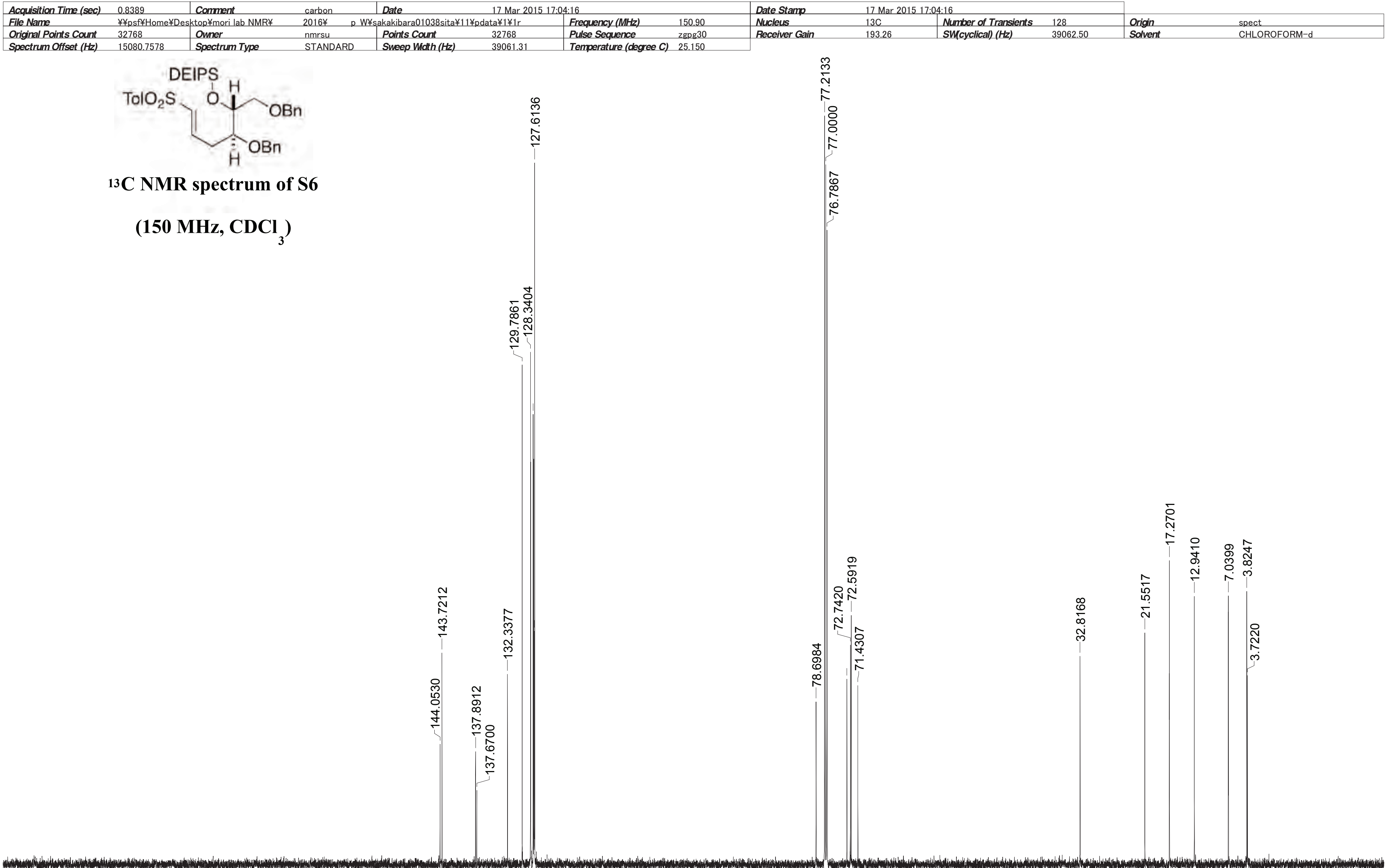

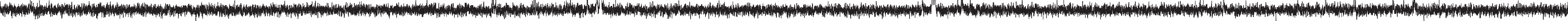




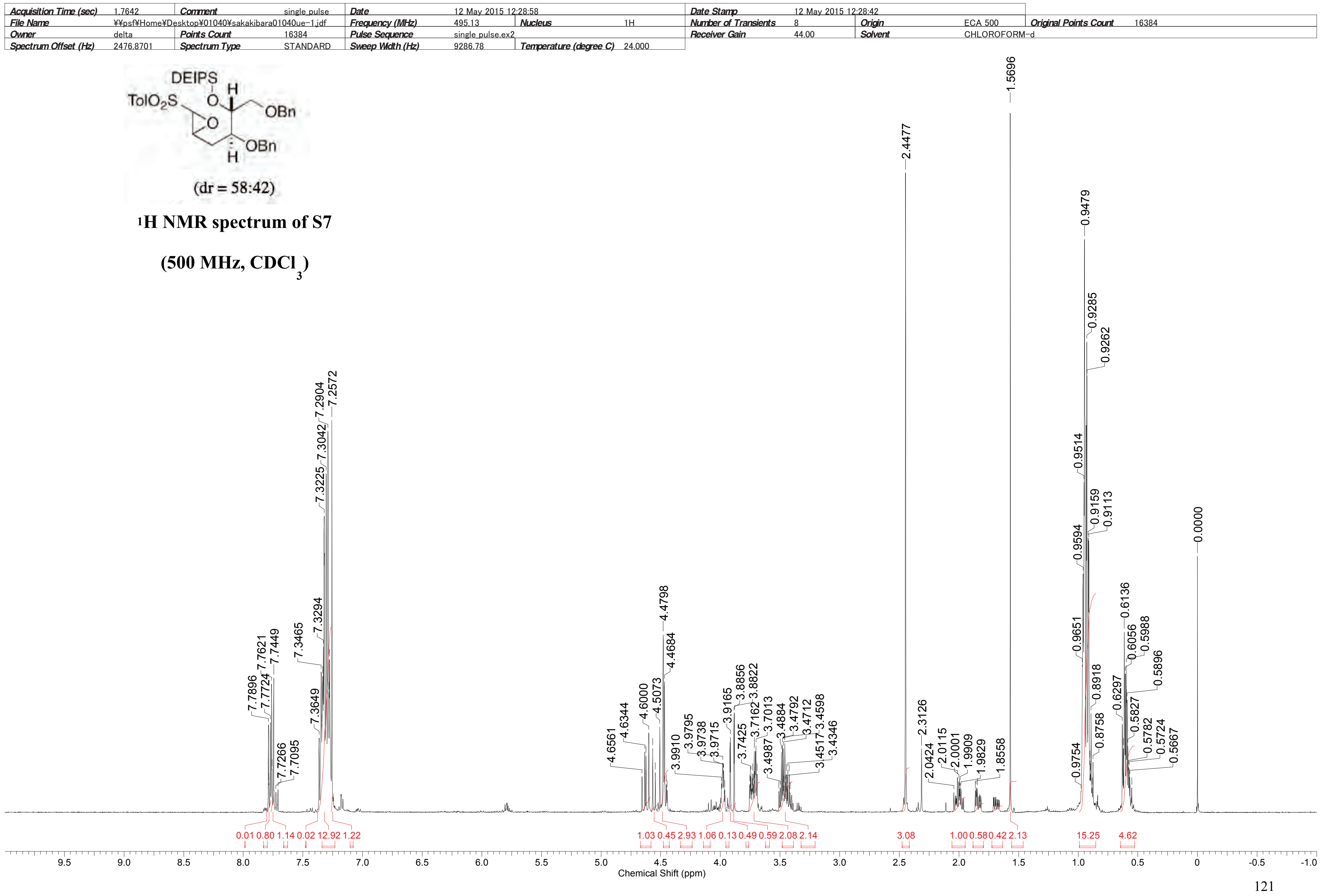


This report was created by ACD/NMR Processor Academic Edition. For more information go to www.acdlabs.com/nmrproc/

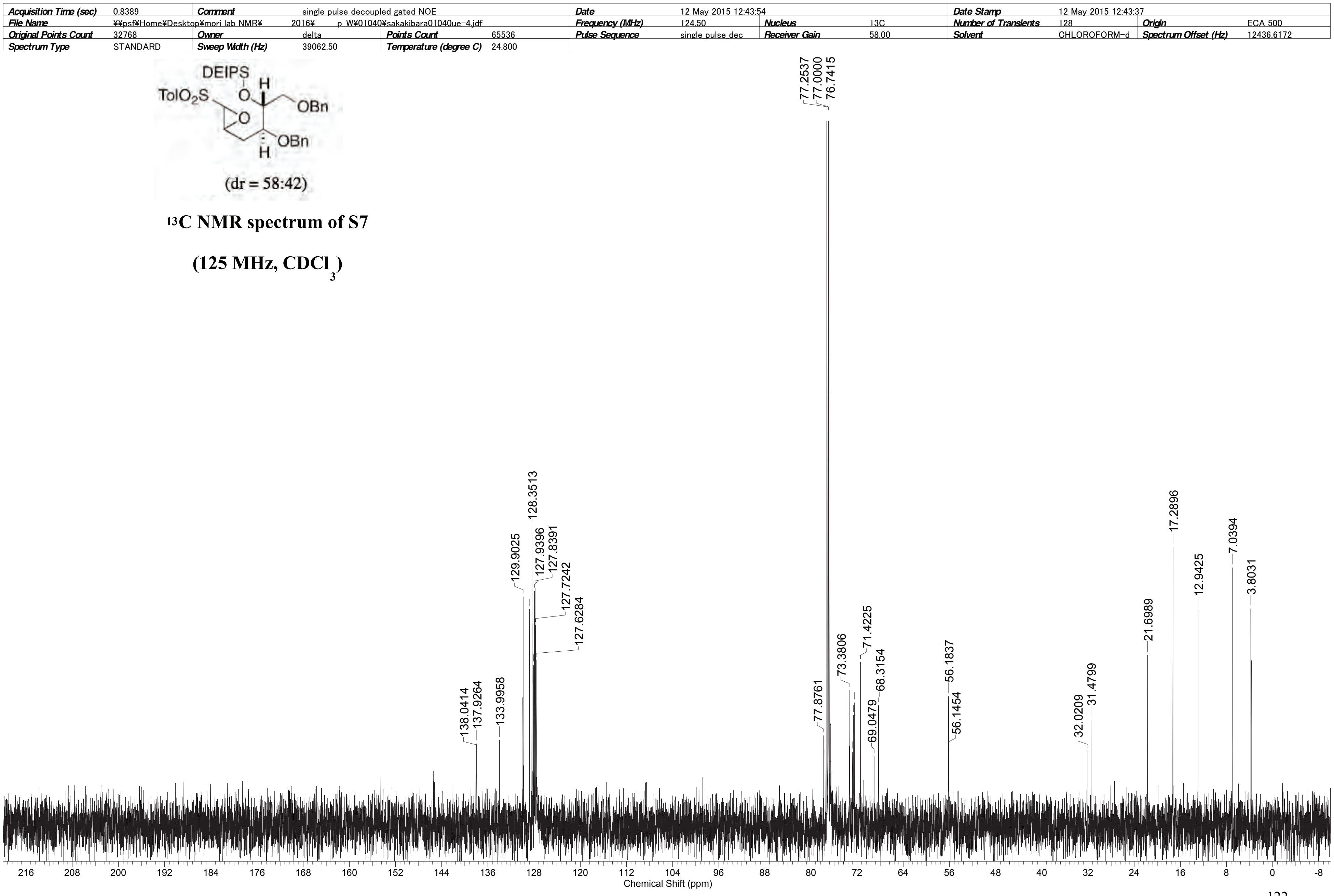


This report was created by ACD/NMR Processor Academic Edition. For more information go to www.acdlabs.com/nmrproc/

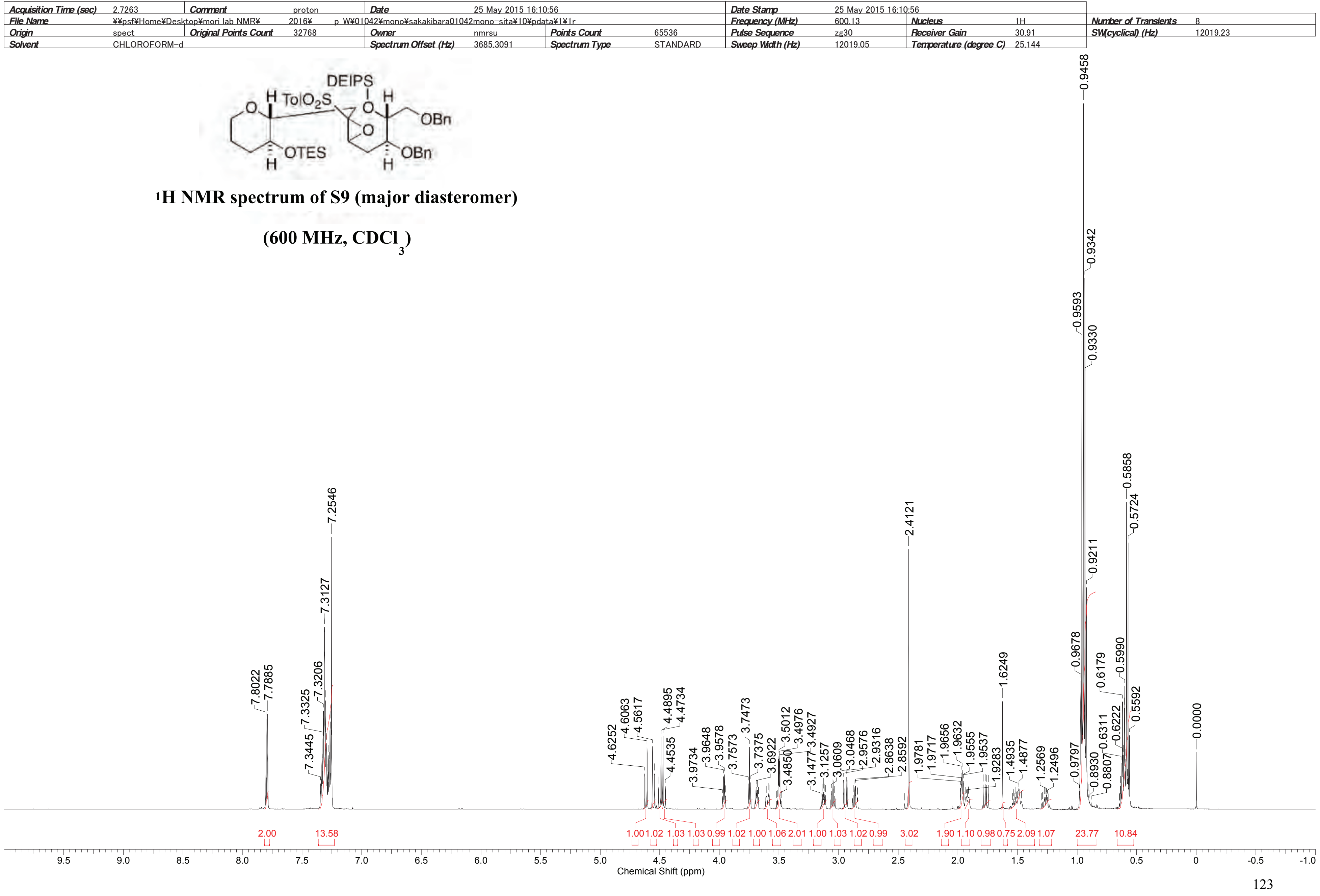


This report was created by ACD/NMR Processor Academic Edition. For more information go to www.acdlabs.com/nmrproc/

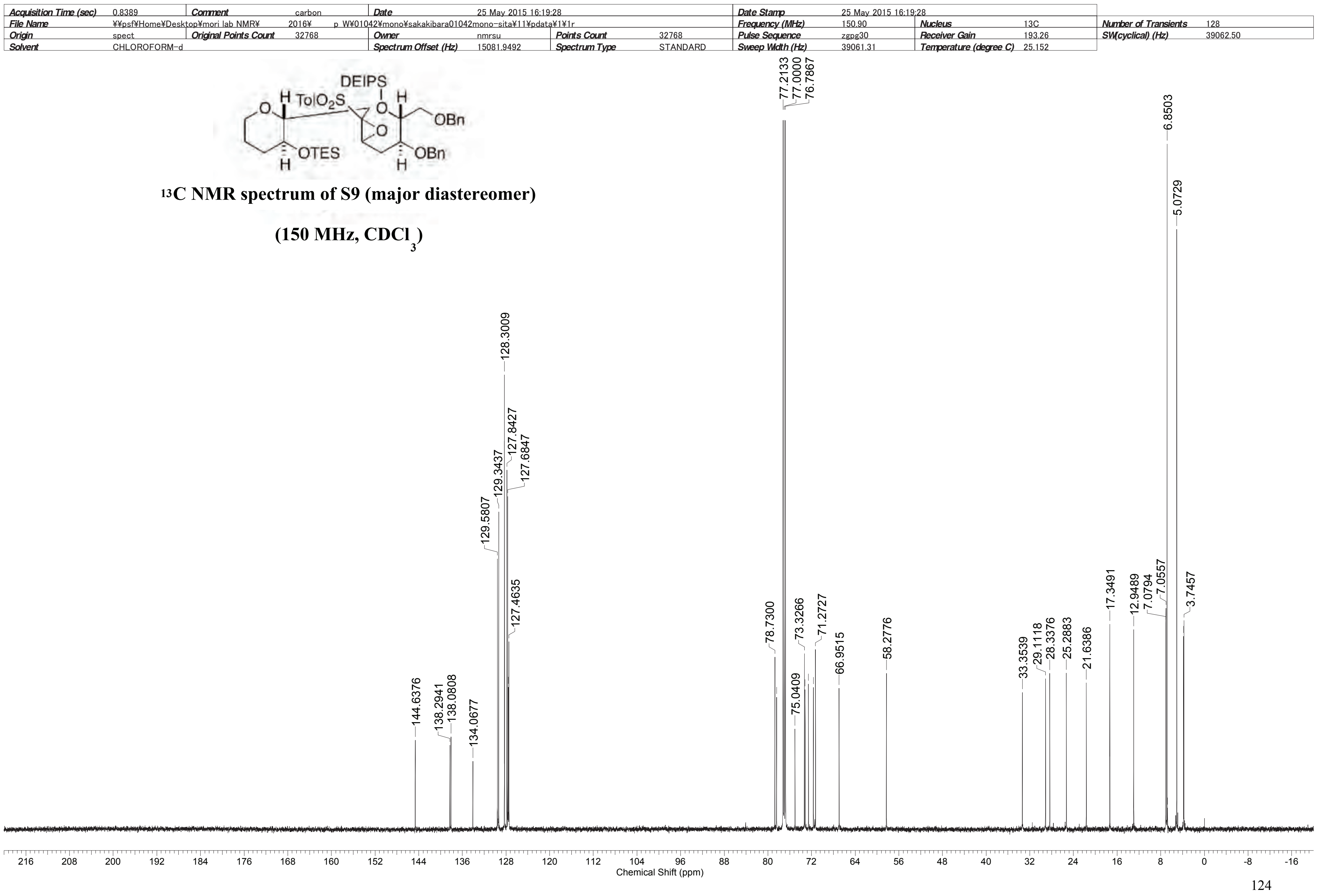




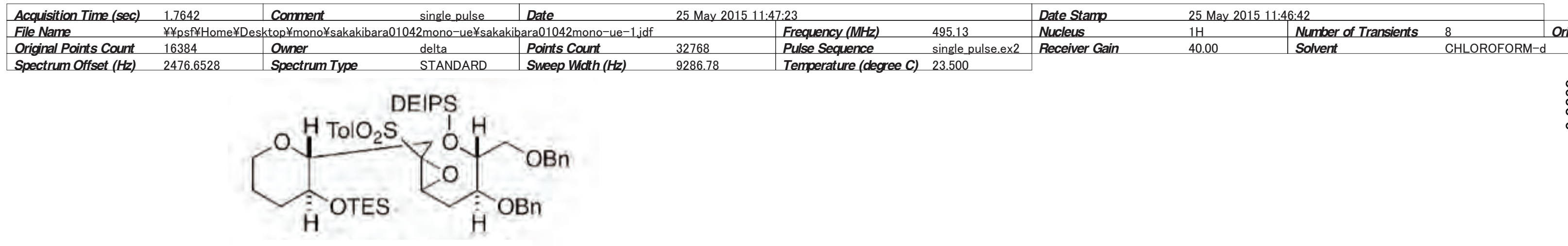

1H NMR spectrum of S9 (minor diastereomer)

(500 $\mathrm{MHz}_{2} \mathrm{CDCl}_{3}$ )

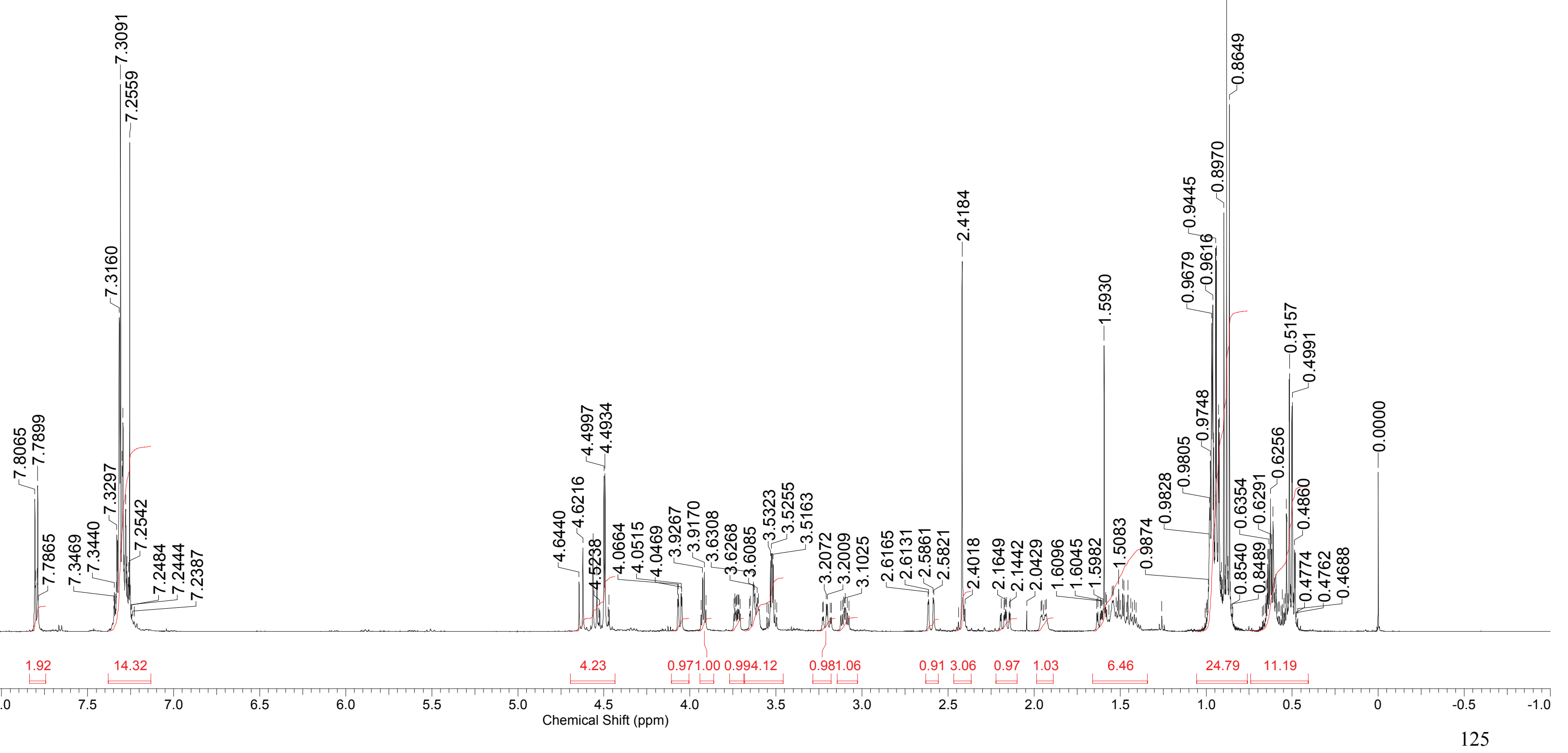




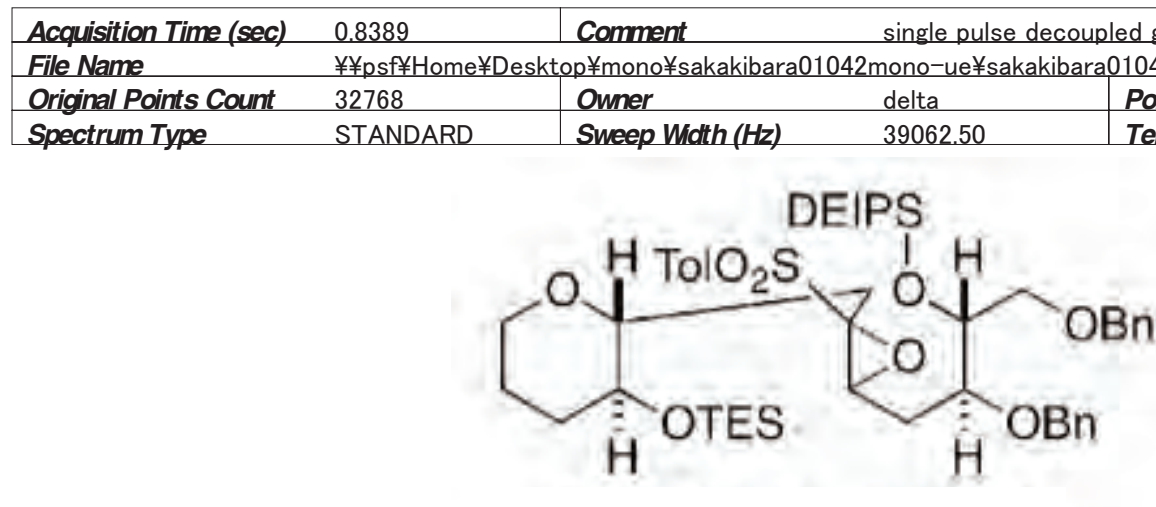

13C NMR spectrum of S9 (minor diasteromer)

(125 MHz, CDCl ) $_{3}$

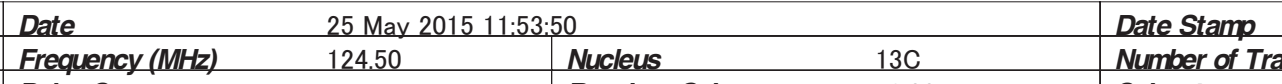
Frequency (MHz)
Pulse Sequence Nucleus
Receiver G

¿



尺्:

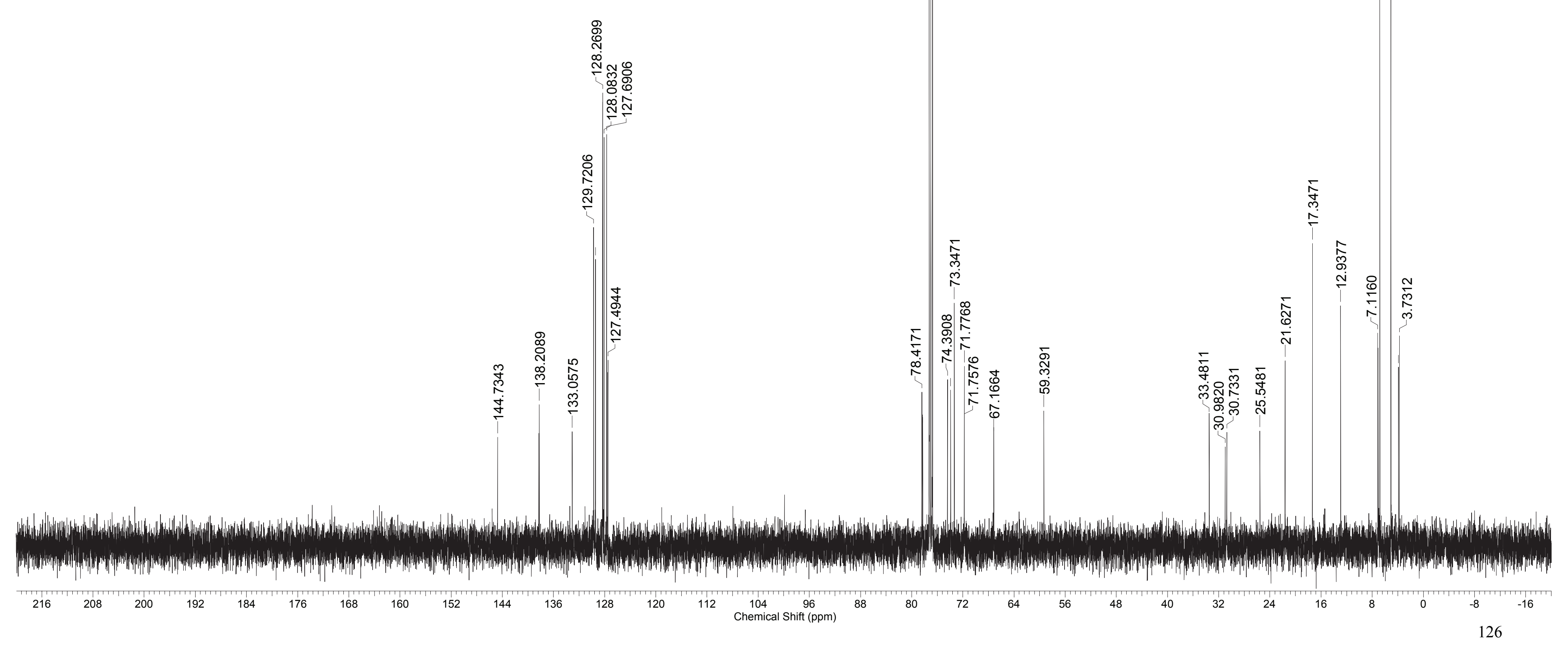
$\begin{array}{ll}\text { Origin } & \text { ECA } 500 \\ \text { Spectrum Offset (Hz) } & 12436.6172\end{array}$ 
This report was created by ACD/NMR Processor Academic Edition. For more information go to www.acdlabs.com/nmrproc/

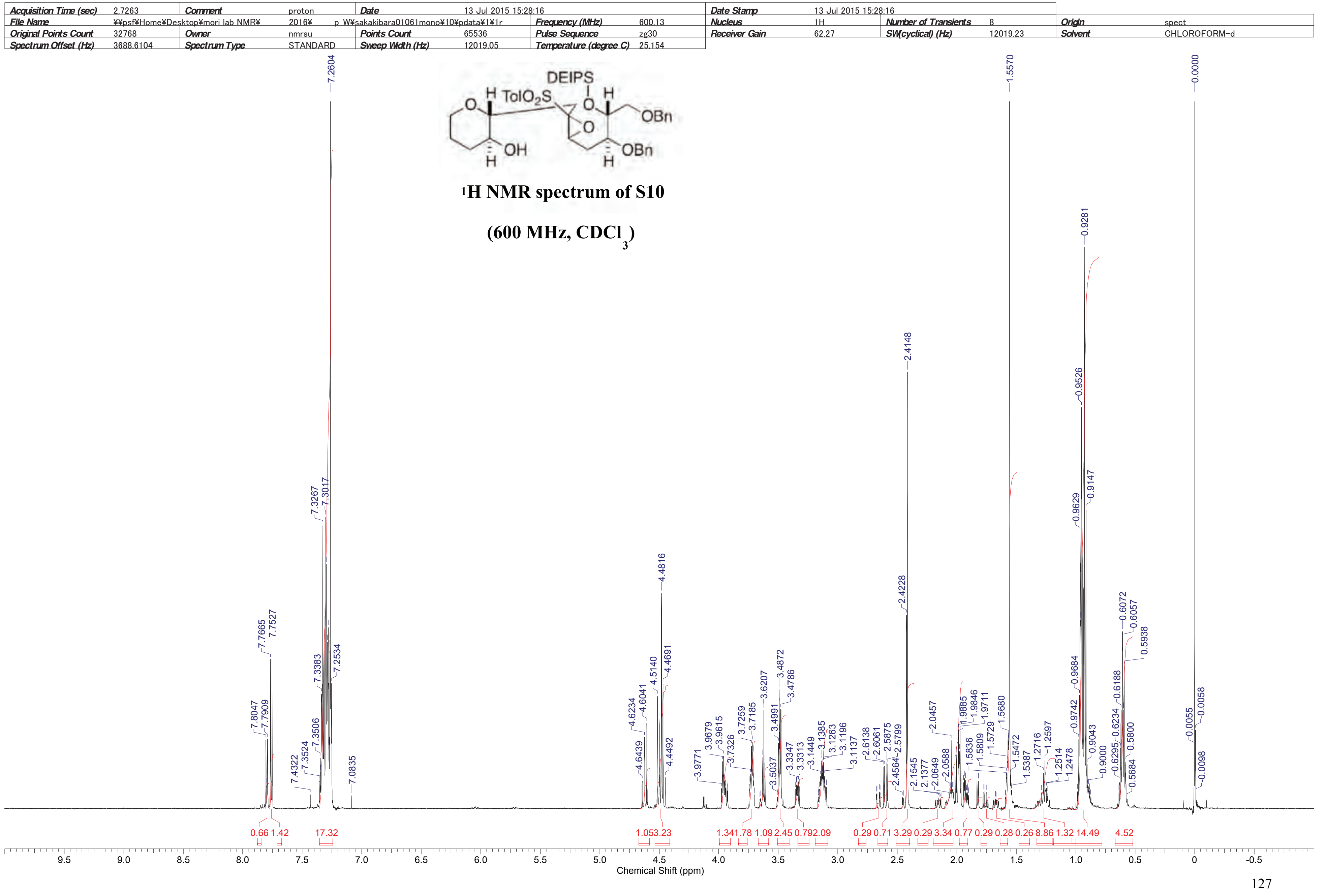


This report was created by ACD/NMR Processor Academic Edition. For more information go to www.acdlabs.com/nmrproc/

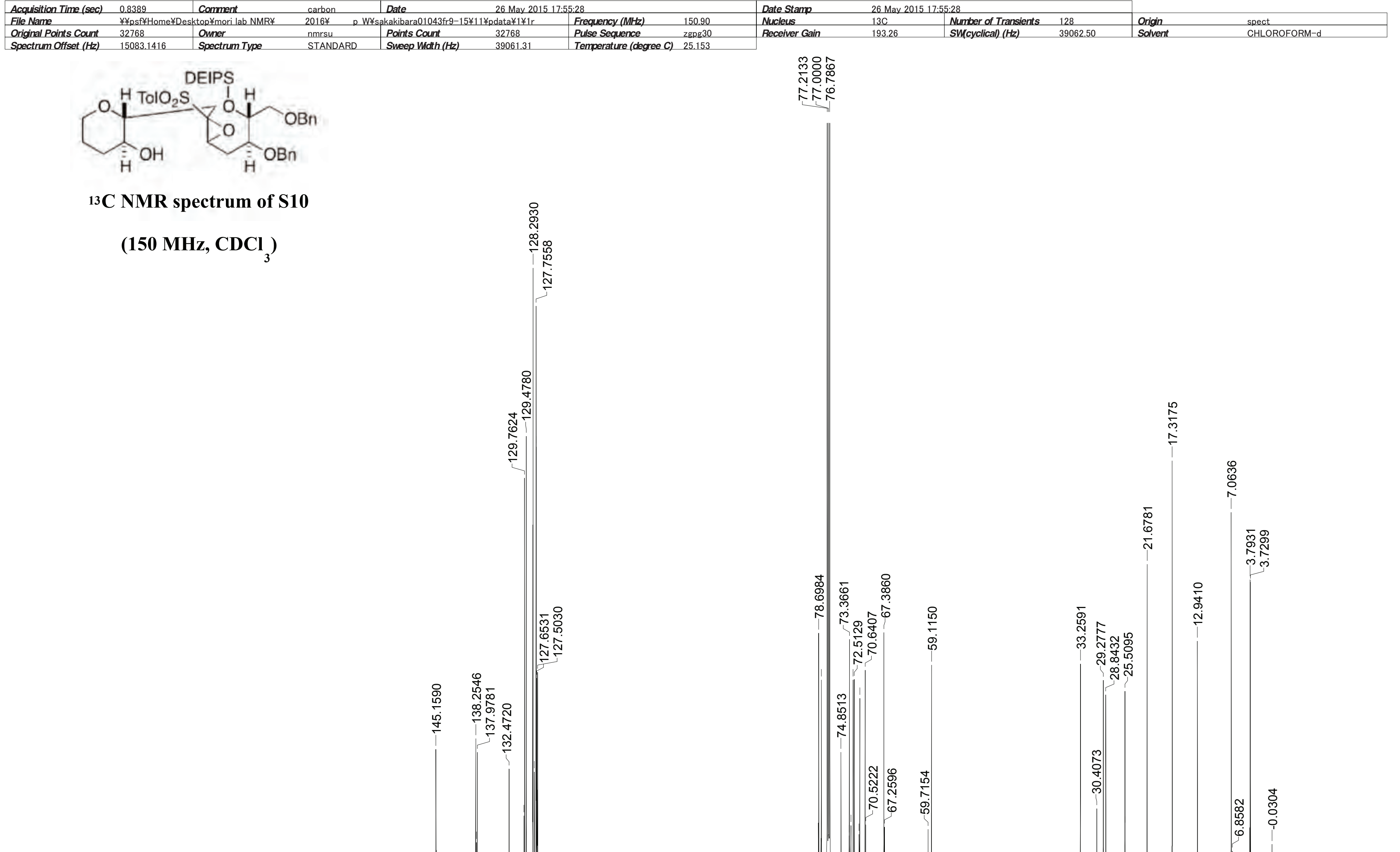


This report was created by ACD/NMR Processor Academic Edition. For more information go to www.acdlabs.com/nmrproc/

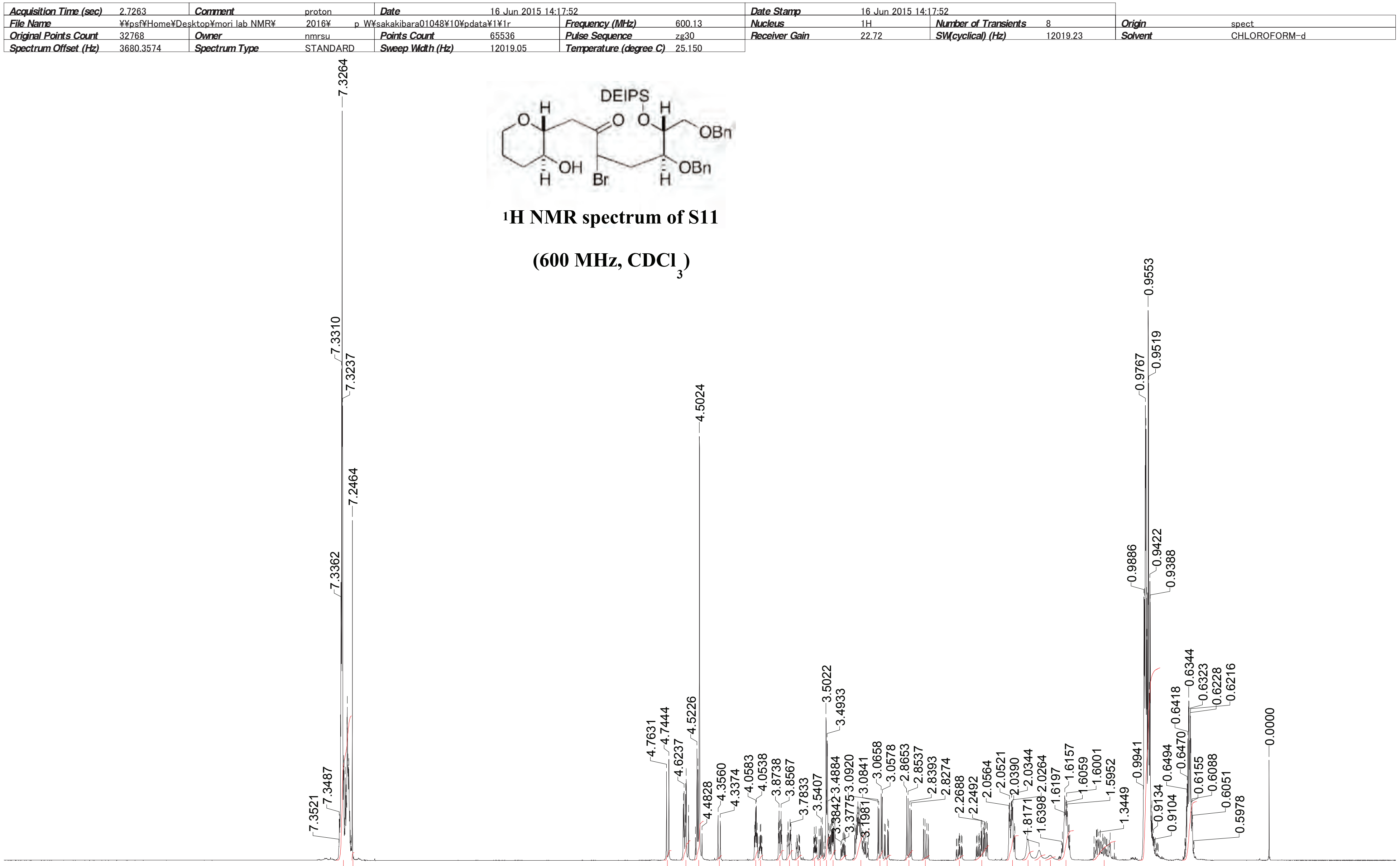


This report was created by ACD/NMR Processor Academic Edition. For more information go to www.acdlabs.com/nmrproc/

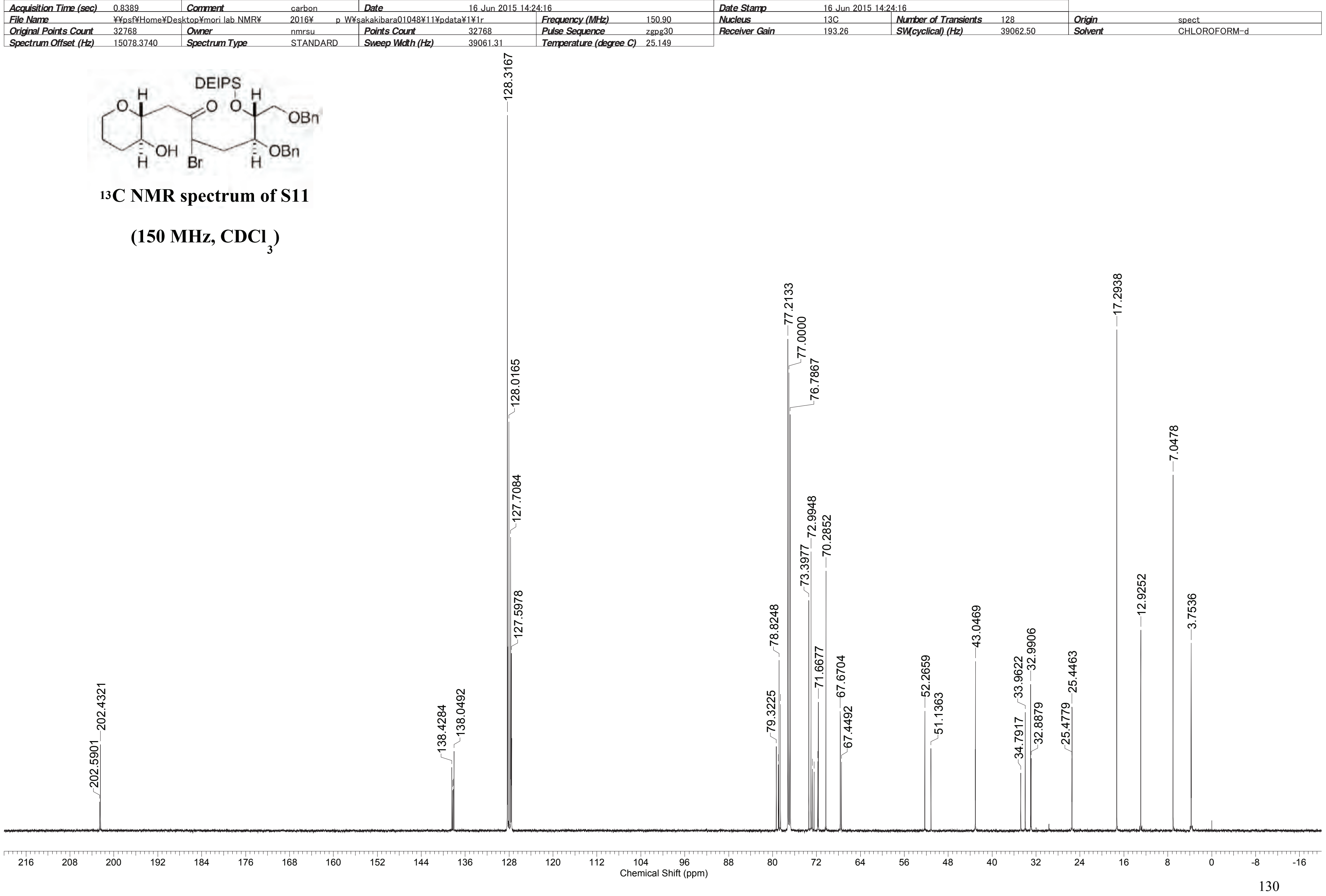


This report was created by ACD/NMR Processor Academic Edition. For more information go to www.acdlabs.com/nmrproc/

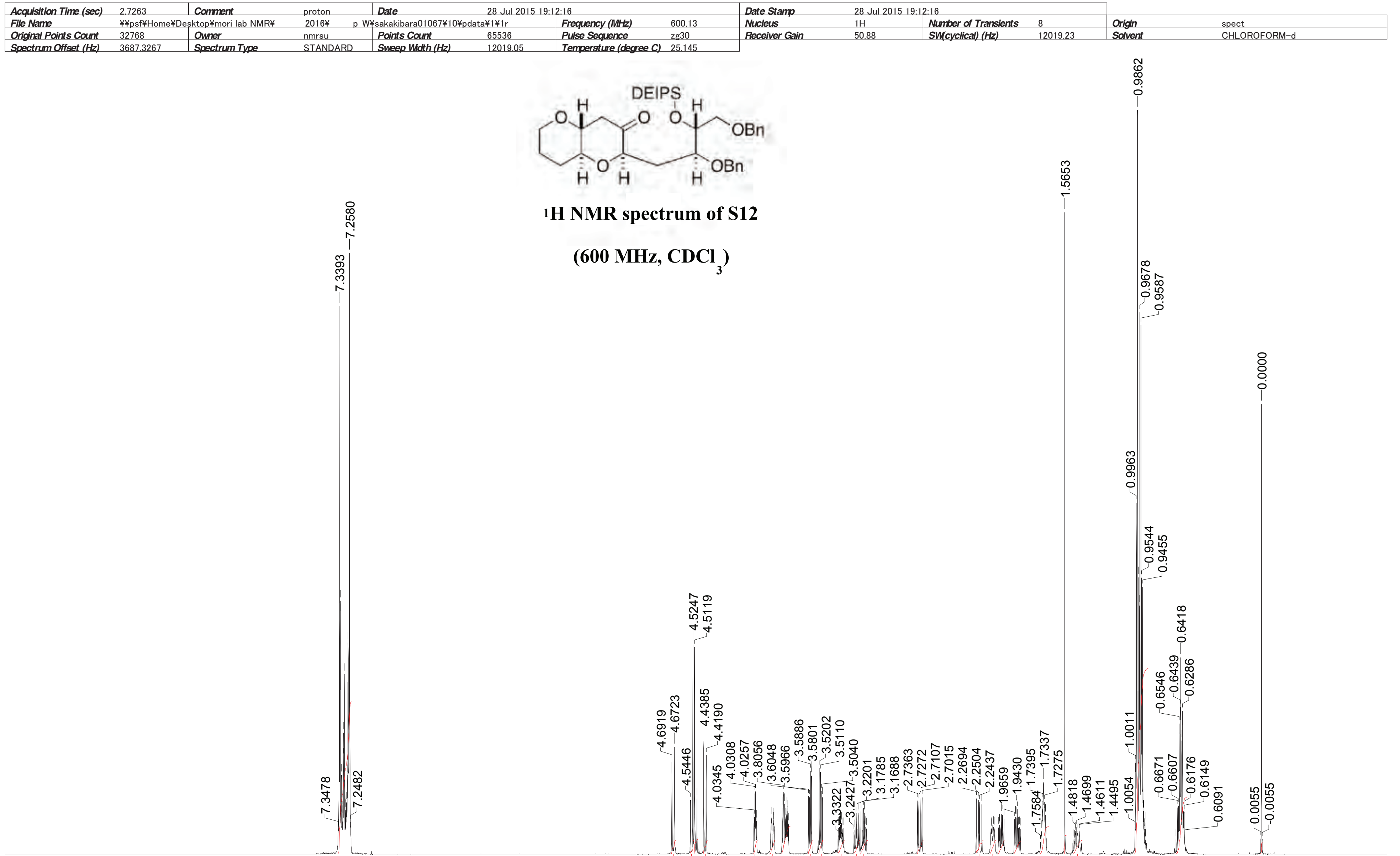

11.28

1.00 1.041.10 0.991.00 1.04 2.050.99 1.051.001.011.00 $0.99 \quad 1.001 .011 .050 .992 .031 .401 .04$ 
This report was created by ACD/NMR Processor Academic Edition. For more information go to www.acdlabs.com/nmrproc/

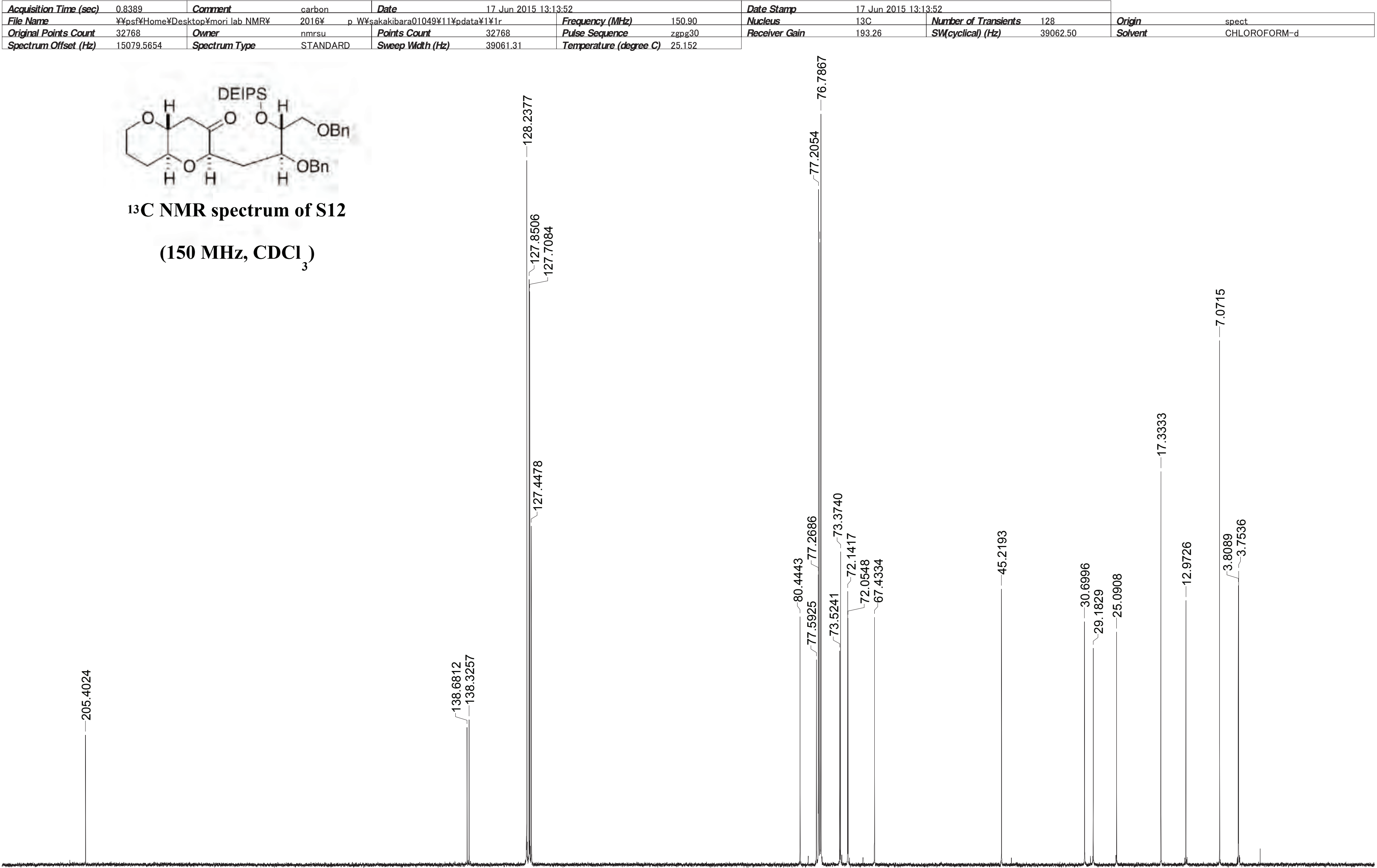


This report was created by ACD/NMR Processor Academic Edition. For more information go to www.acdlabs.com/nmrproc/

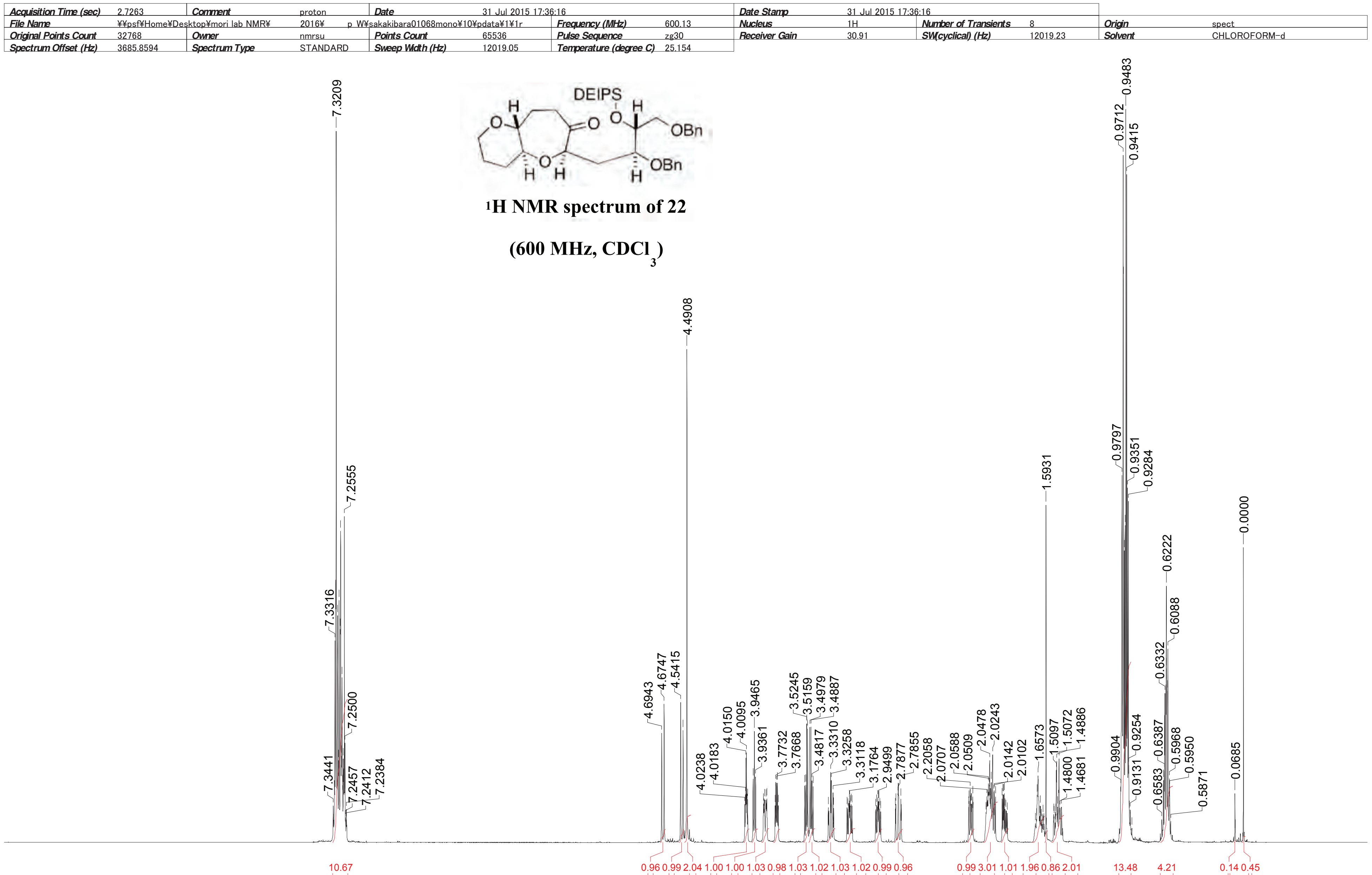


This report was created by ACD/NMR Processor Academic Edition. For more information go to www.acdlabs.com/nmrproc/

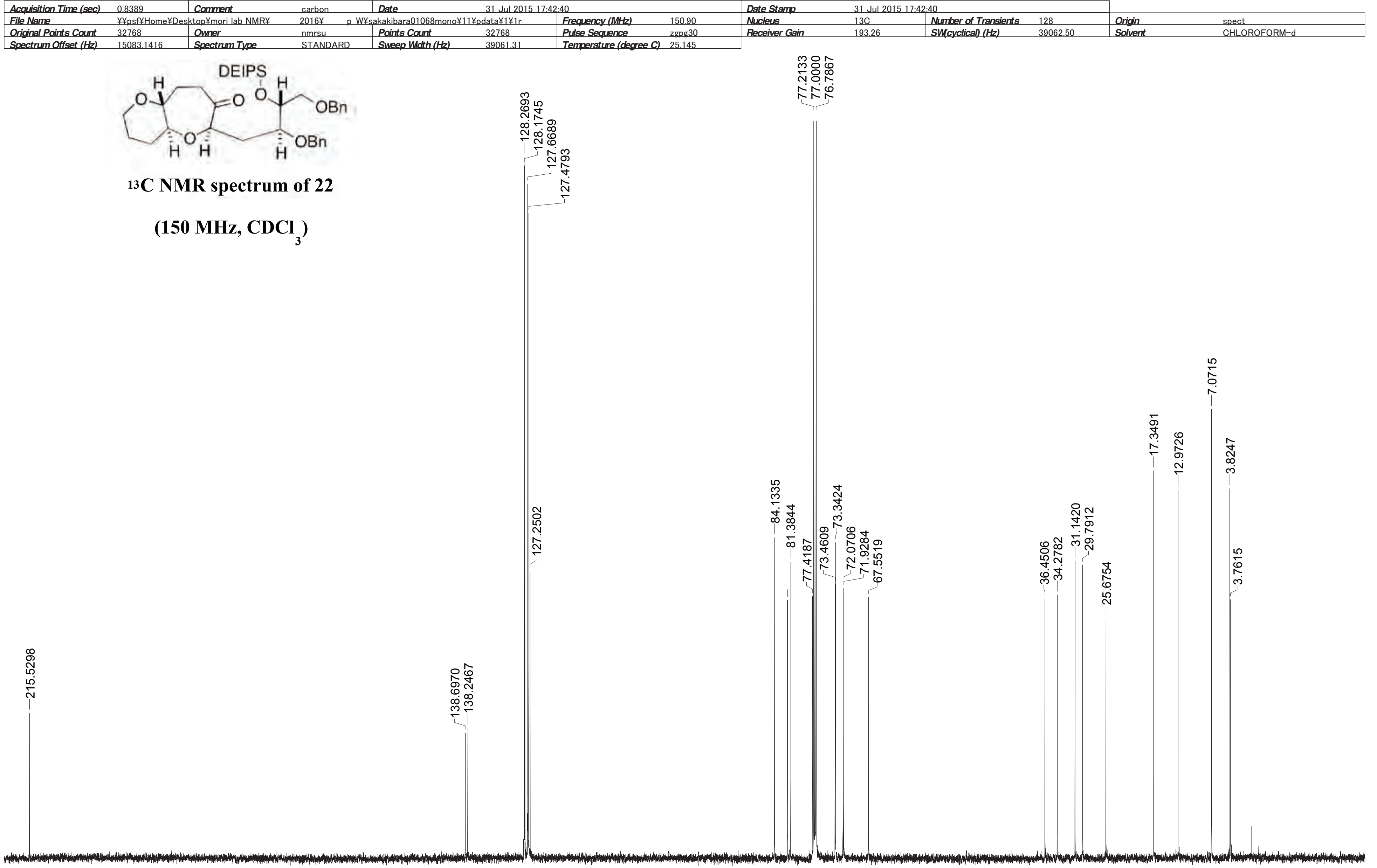


This report was created by ACD/NMR Processor Academic Edition. For more information go to www.acdlabs.com/nmrproc/

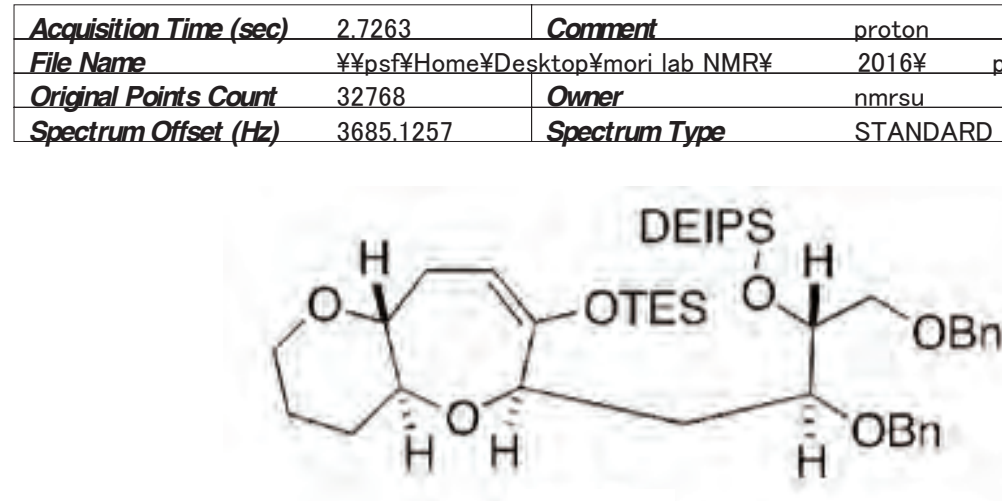

1H NMR spectrum of S13

(600 MHz, CDCl $\left.{ }_{3}\right)$

\begin{tabular}{ll}
\hline Frequency (MHz) & 600.13 \\
PPllse Sequence & z390 \\
Temperature (degree C) & 25.144
\end{tabular}

Date Stamp
Nucleus
Rect

Nucleus 17 Aug 2015 17:59:44 7:59:44
Number of Transients
SW(cyclical) (Hz) 12019.23 Origin
Solvent spect
CHLOROFORM-d

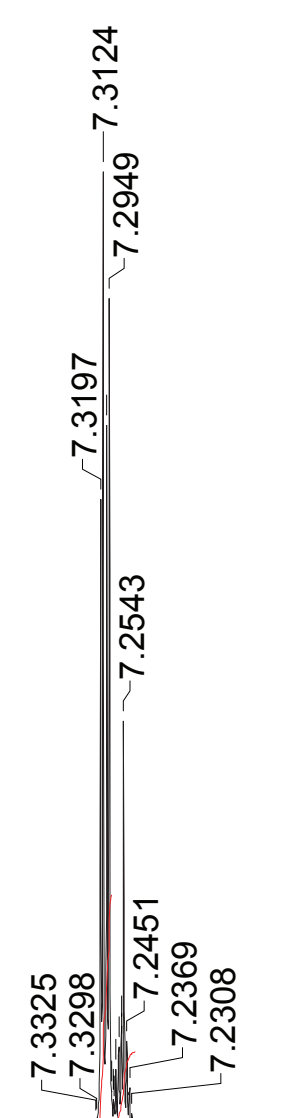

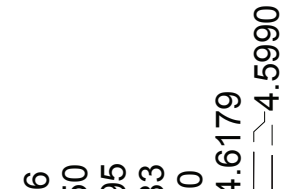

兽

$\frac{d}{2}$

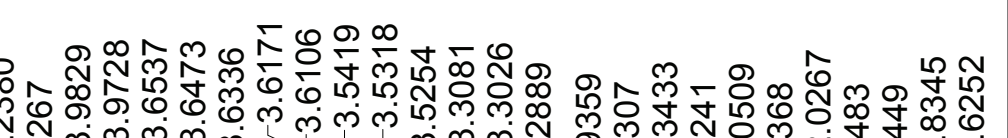

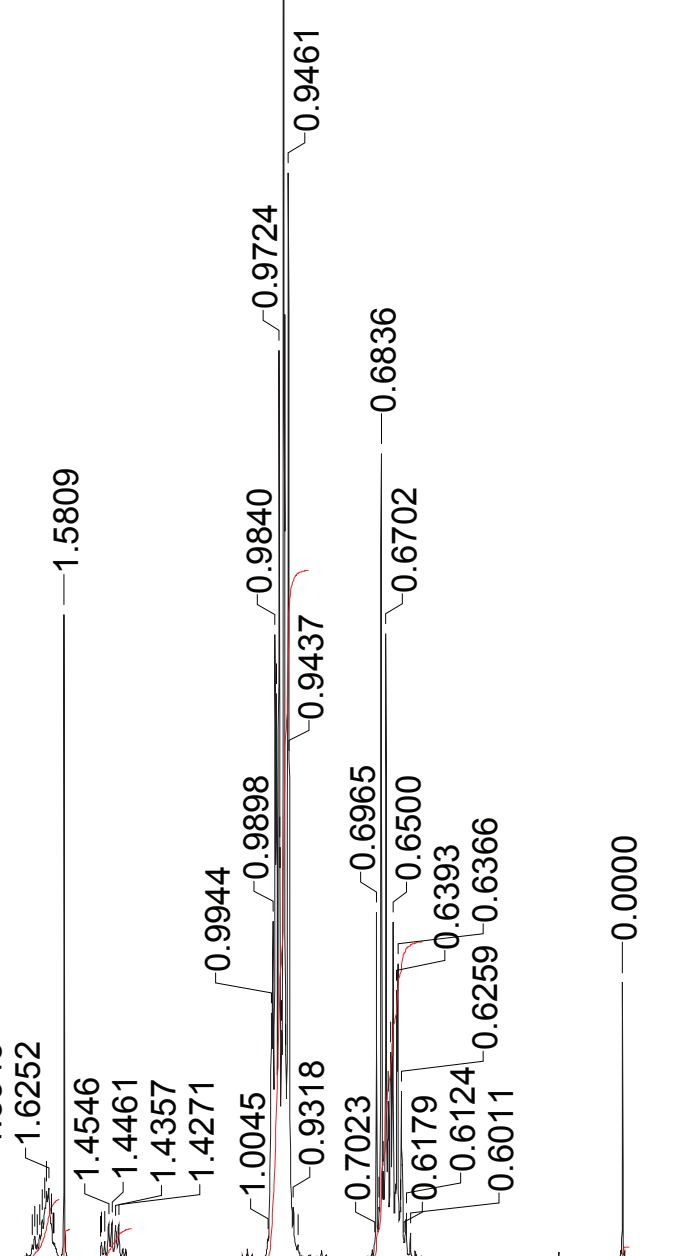

$1.001 .031 .012 .030 .98 \quad 1.001 .022 .001 .032 .04$ 0.991 .001 .011 .020 .982 .011 .021 .04 $22.60 \quad 10.44$ 0.46 
This report was created by ACD/NMR Processor Academic Edition. For more information go to www.acdlabs.com/nmrproc/

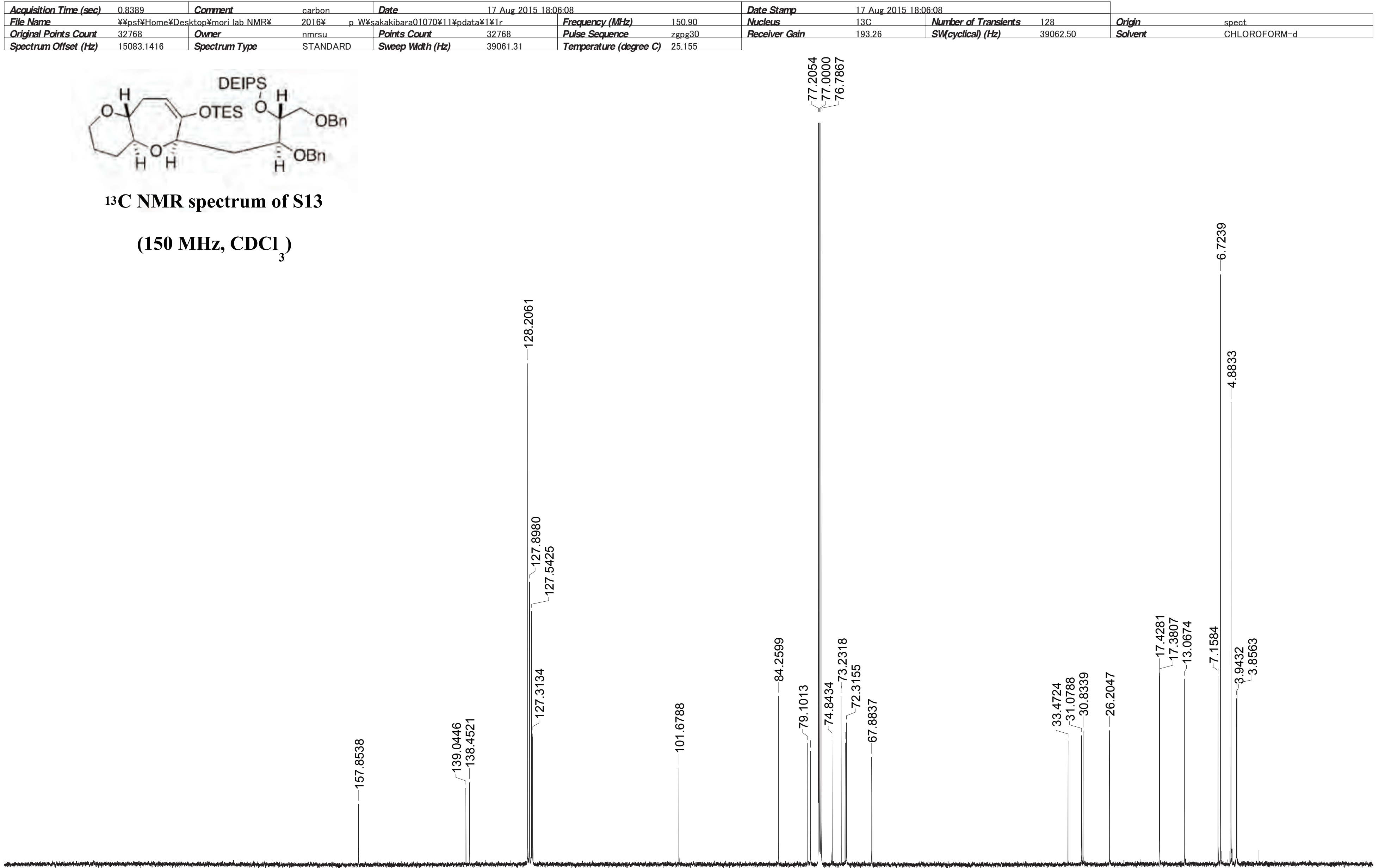


This report was created by ACD/NMR Processor Academic Edition. For more information go to www.acdlabs.com/nmrproc/

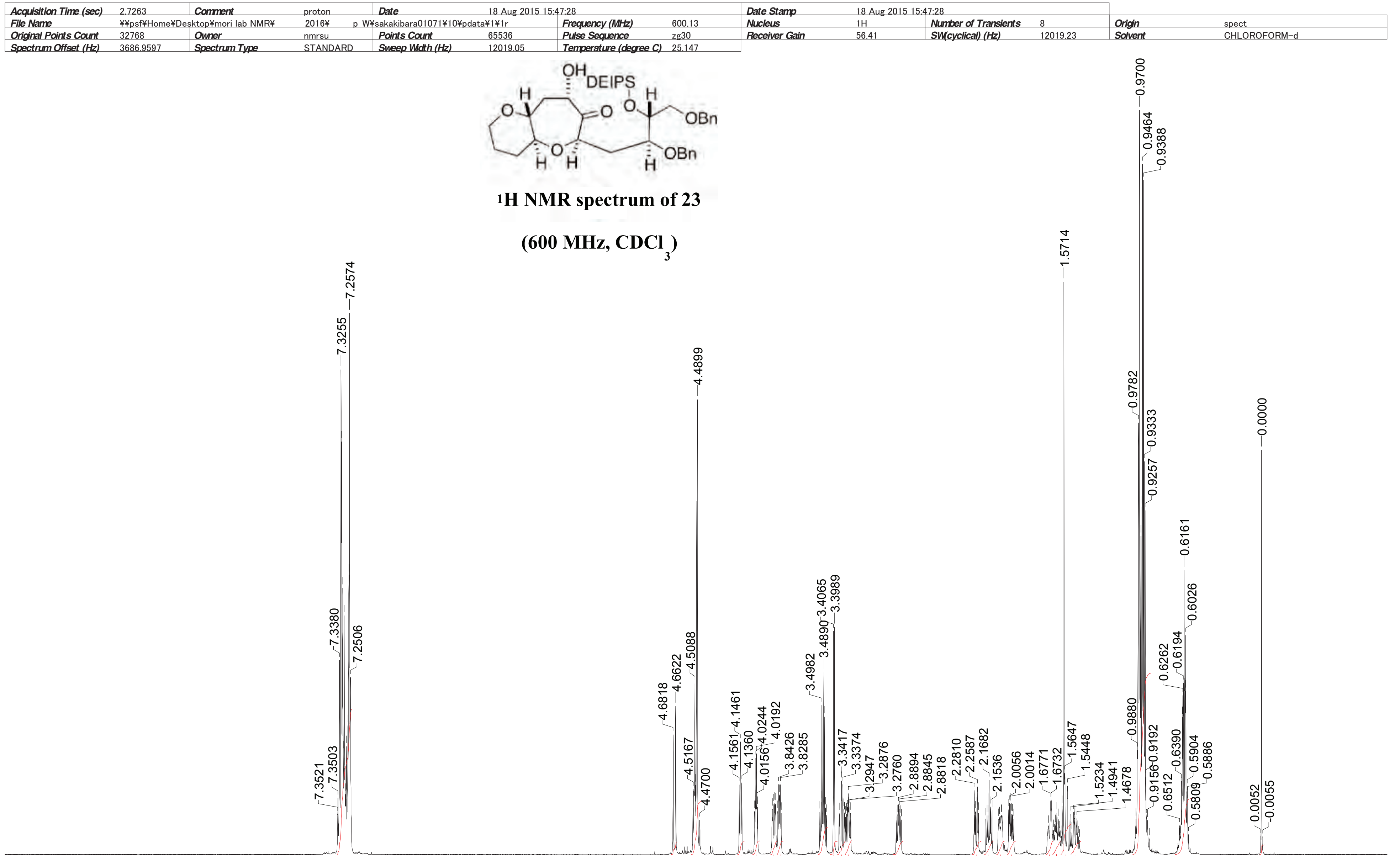

11.56

1.004 .231 .021 .091 .061 .072 .181 .061 .081 .111 .03 1.031 .071 .061 .061 .071 .022 .431 .03 
This report was created by ACD/NMR Processor Academic Edition. For more information go to www.acdlabs.com/nmrproc/

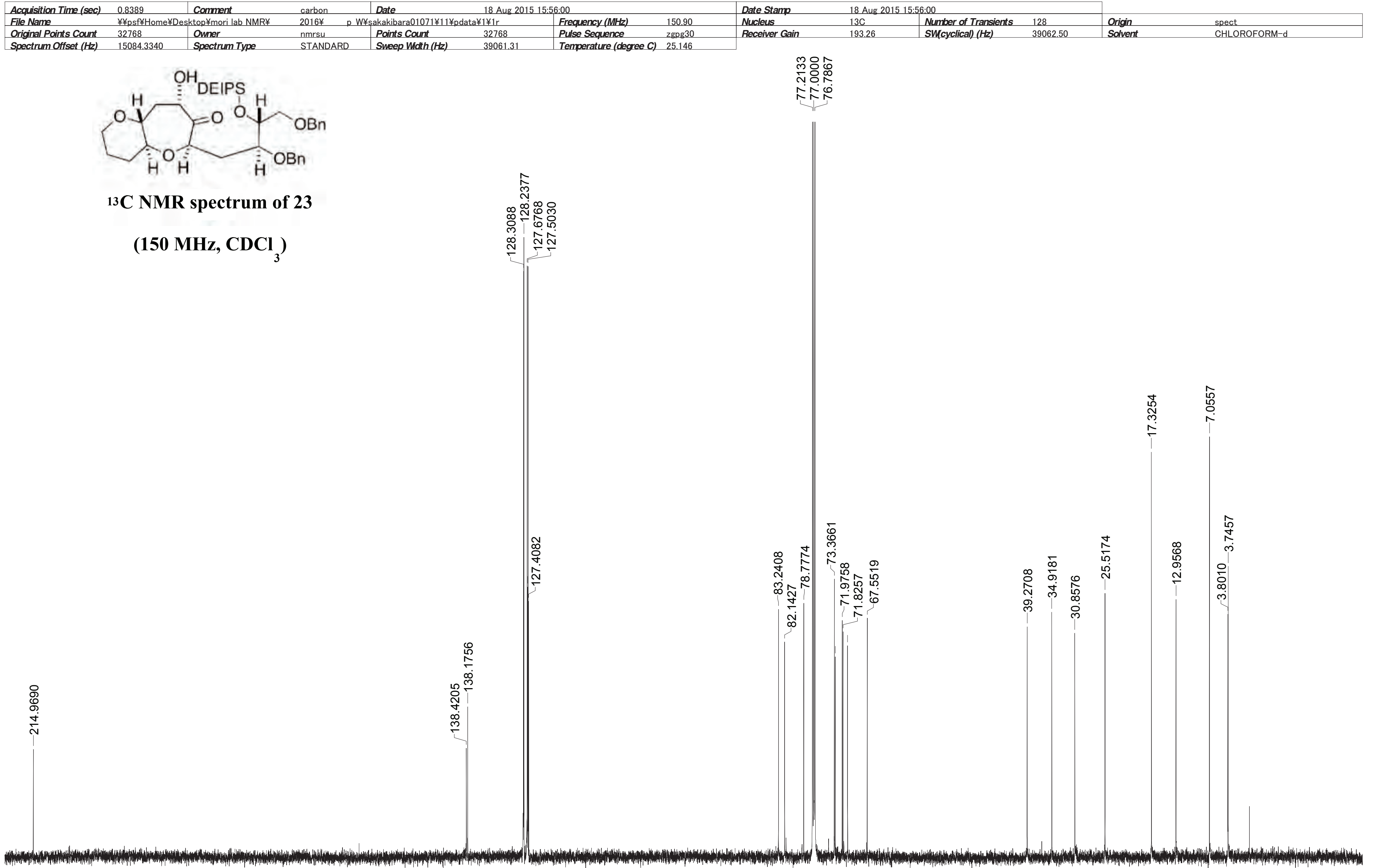

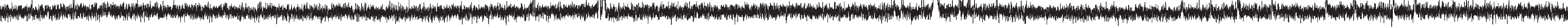


This report was created by ACD/NMR Processor Academic Edition. For more information go to www.acdlabs.com/nmrproc/

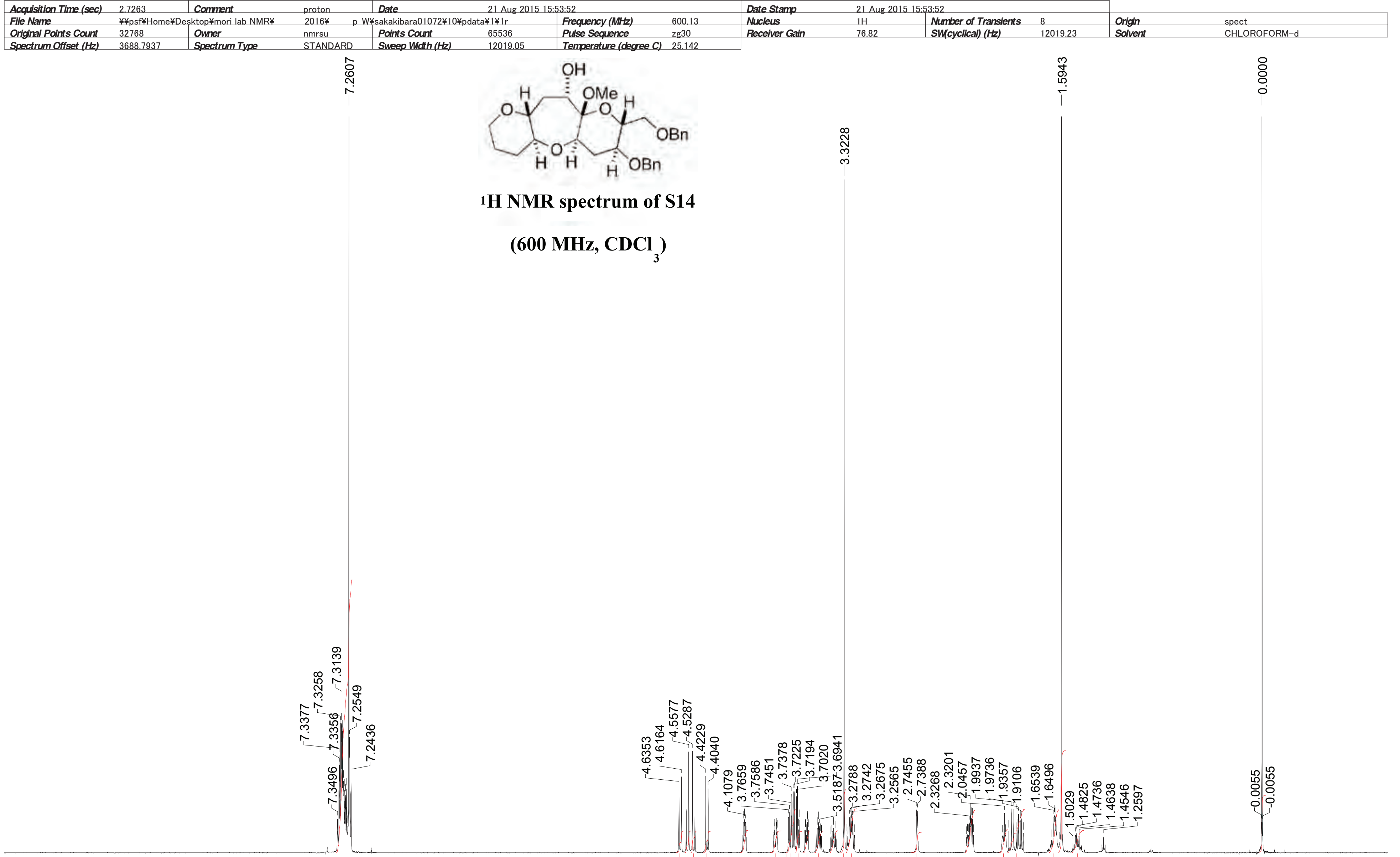

12.78

1.01 1.030.99 1.00 1.06 1.020.002.040.011.00 1.021.01 1.032.98 2.050.96 1.98 1.112.052.024.811.05 
This report was created by ACD/NMR Processor Academic Edition. For more information go to www.acdlabs.com/nmrproc/

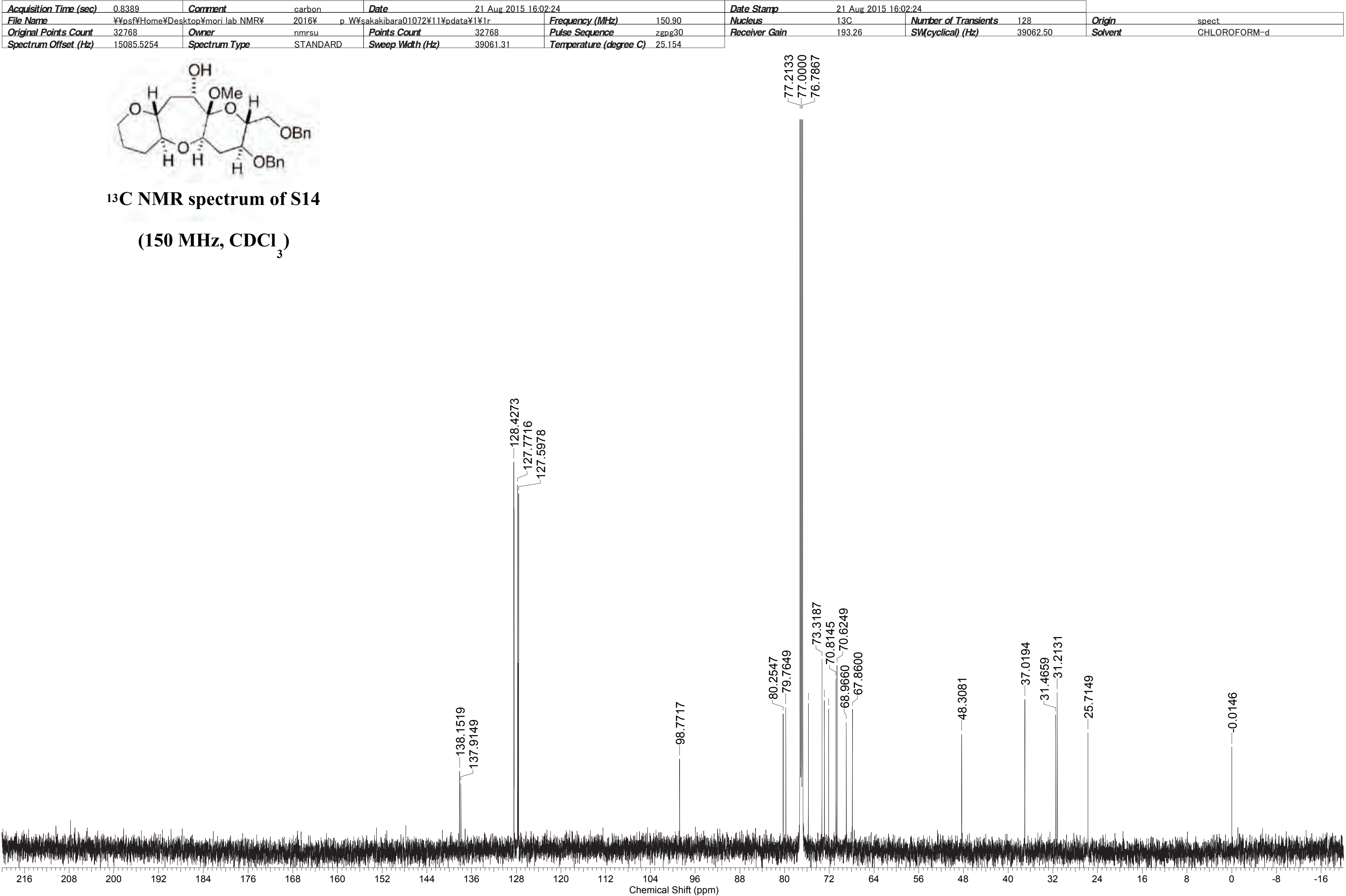


This report was created by ACD/NMR Processor Academic Edition. For more information go to www.acdlabs.com/nmrproc/

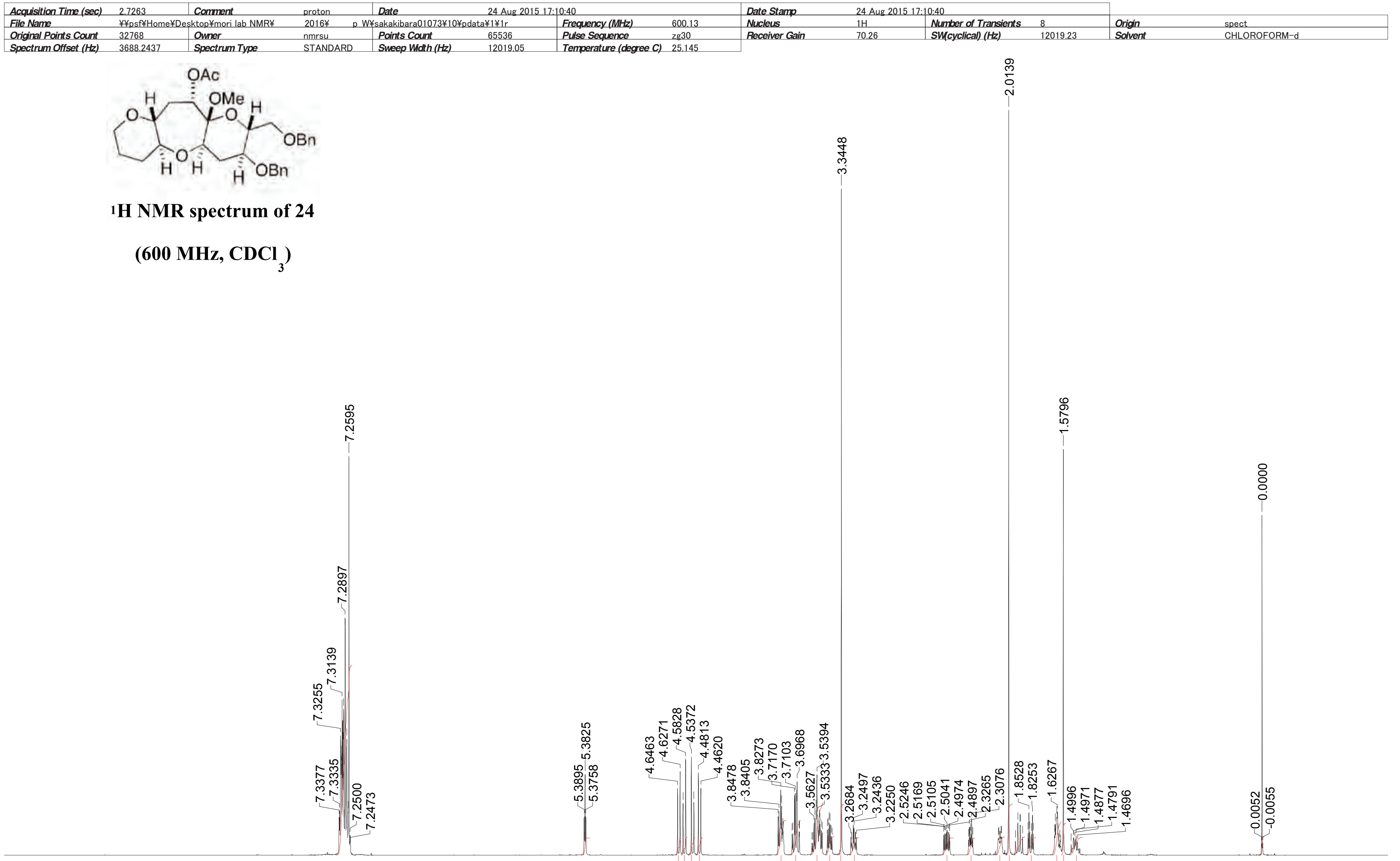

$10.98 \quad 0.99 \quad 0.991 .021 .011 .00 \quad 2.002 .003 .021 .072 .961 .01 \quad 1.000 .991 .062 .961 .051 .001 .951 .851 .03$ 
This report was created by ACD/NMR Processor Academic Edition. For more information go to www.acdlabs.com/nmrproc/

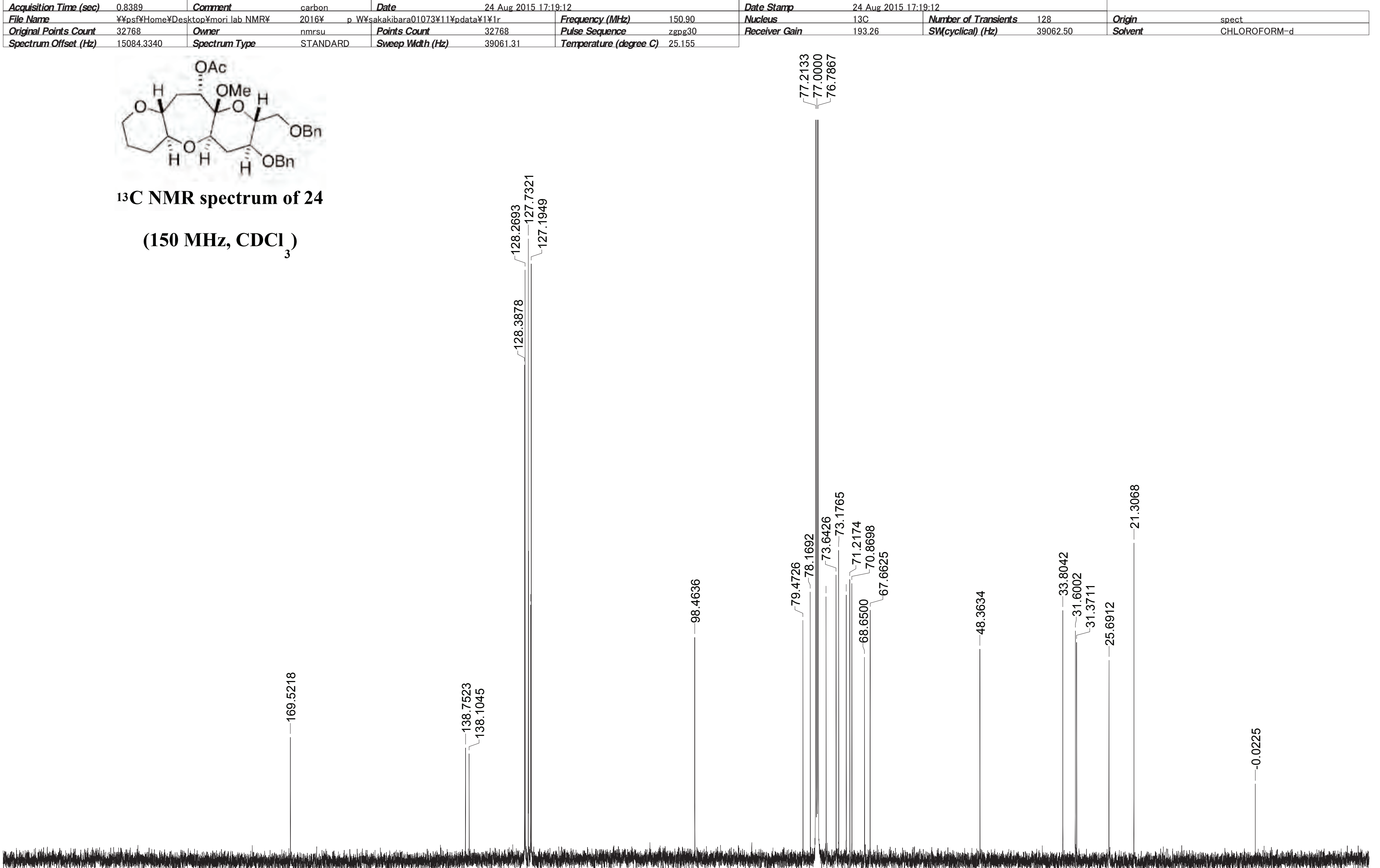


This report was created by ACD/NMR Processor Academic Edition. For more information go to www.acdlabs.com/nmrproc/

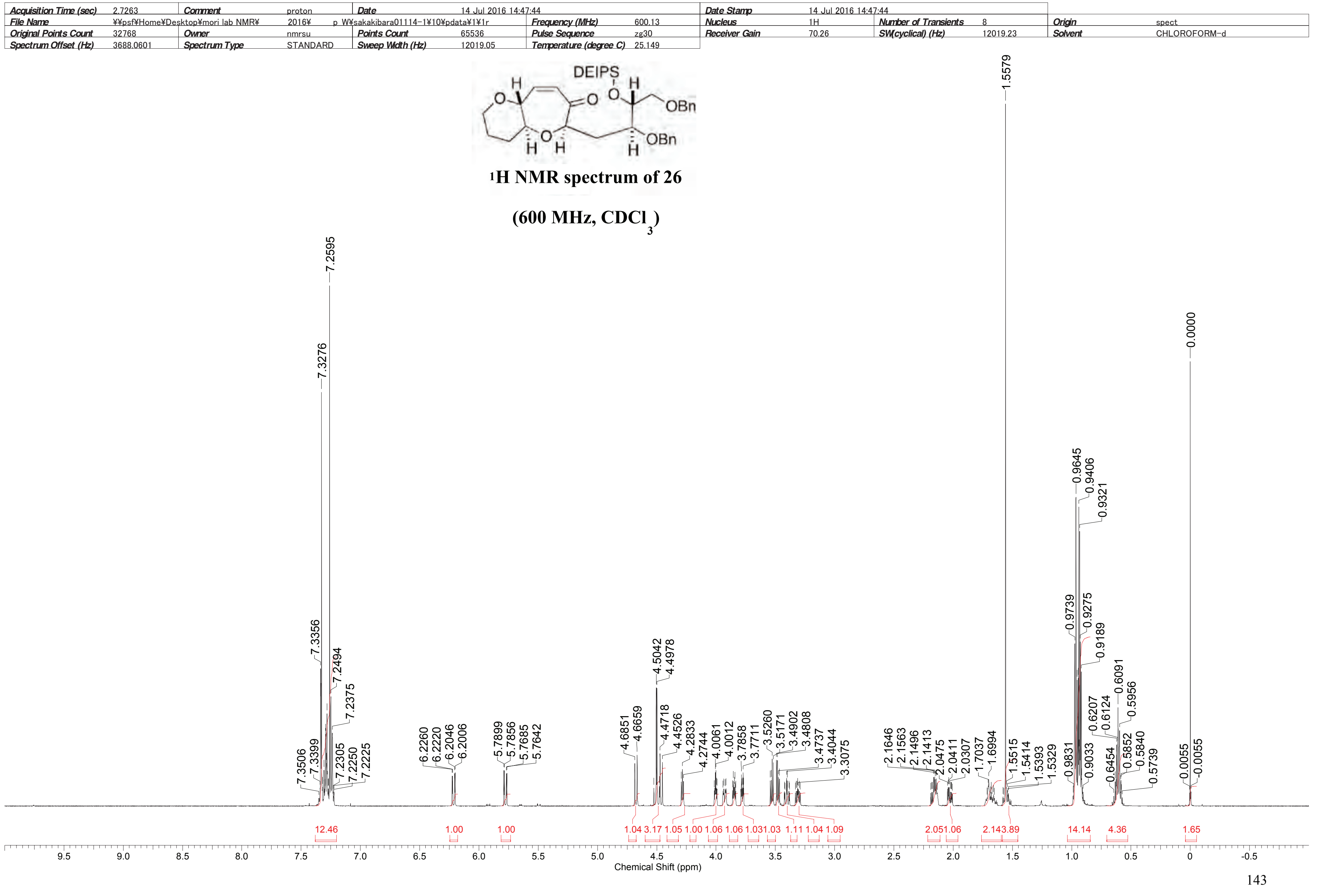


This report was created by ACD/NMR Processor Academic Edition. For more information go to www.acdlabs.com/nmrproc/

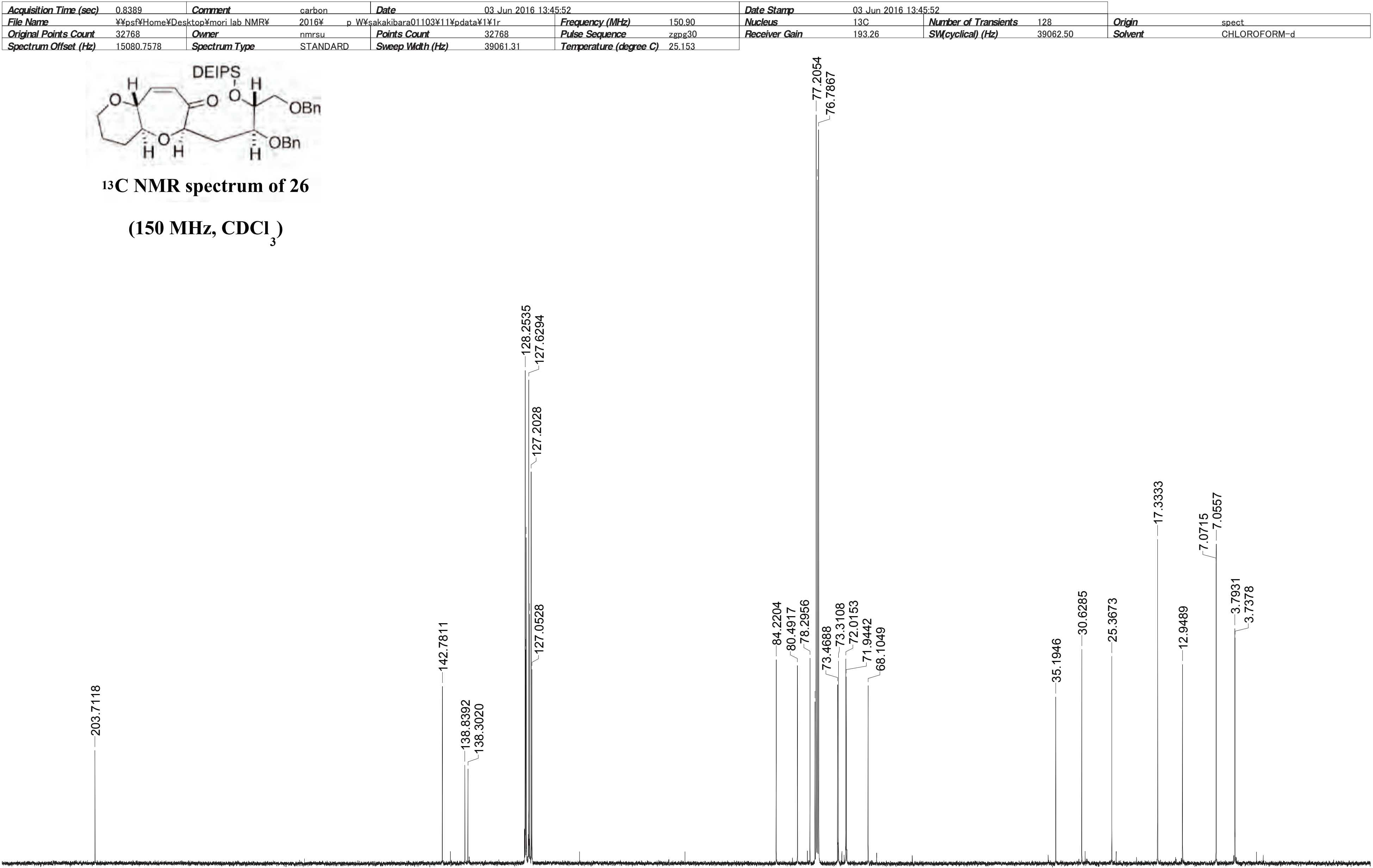


This report was created by ACD/NMR Processor Academic Edition. For more information go to www.acdlabs.com/nmrproc/

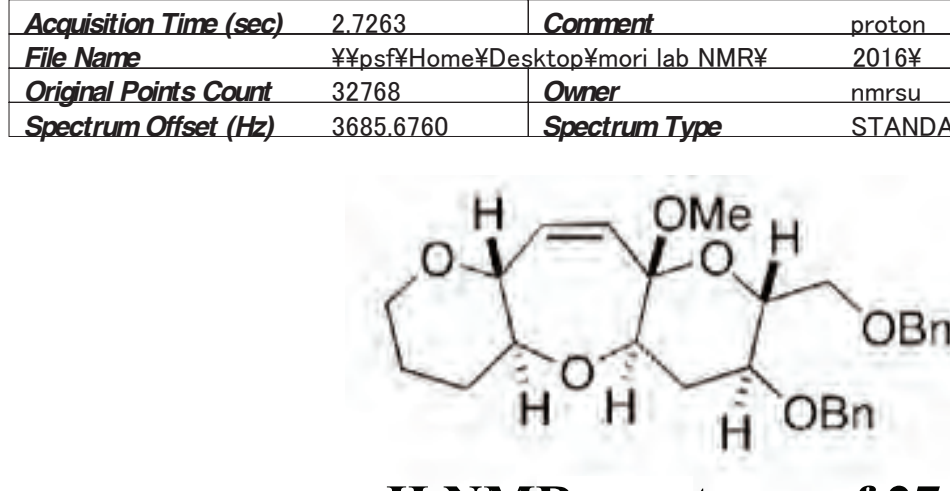

1H NMR spectrum of 27

(600 MHz, CDCl )
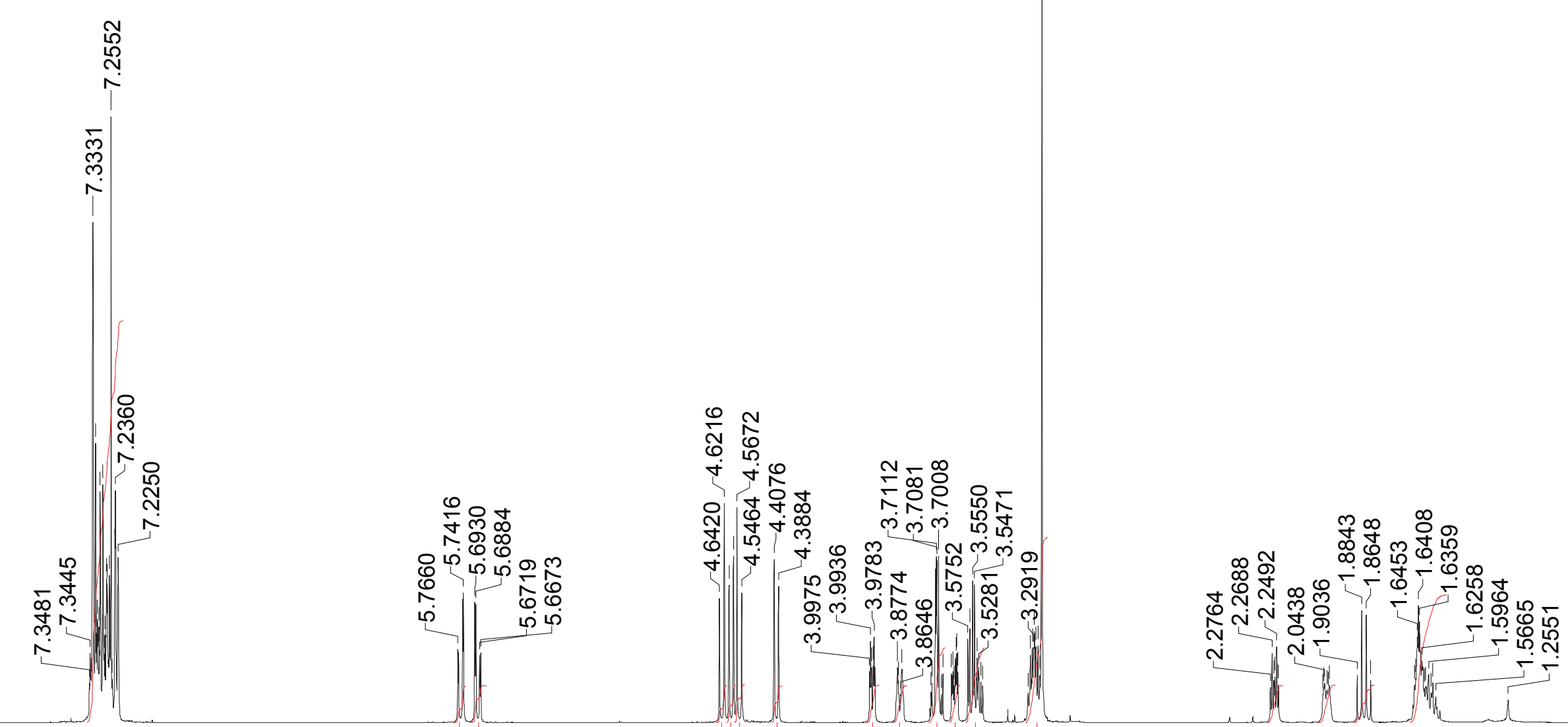

$1.011 .031 .03 \quad 3.5$ 
This report was created by ACD/NMR Processor Academic Edition. For more information go to www.acdlabs.com/nmrproc/

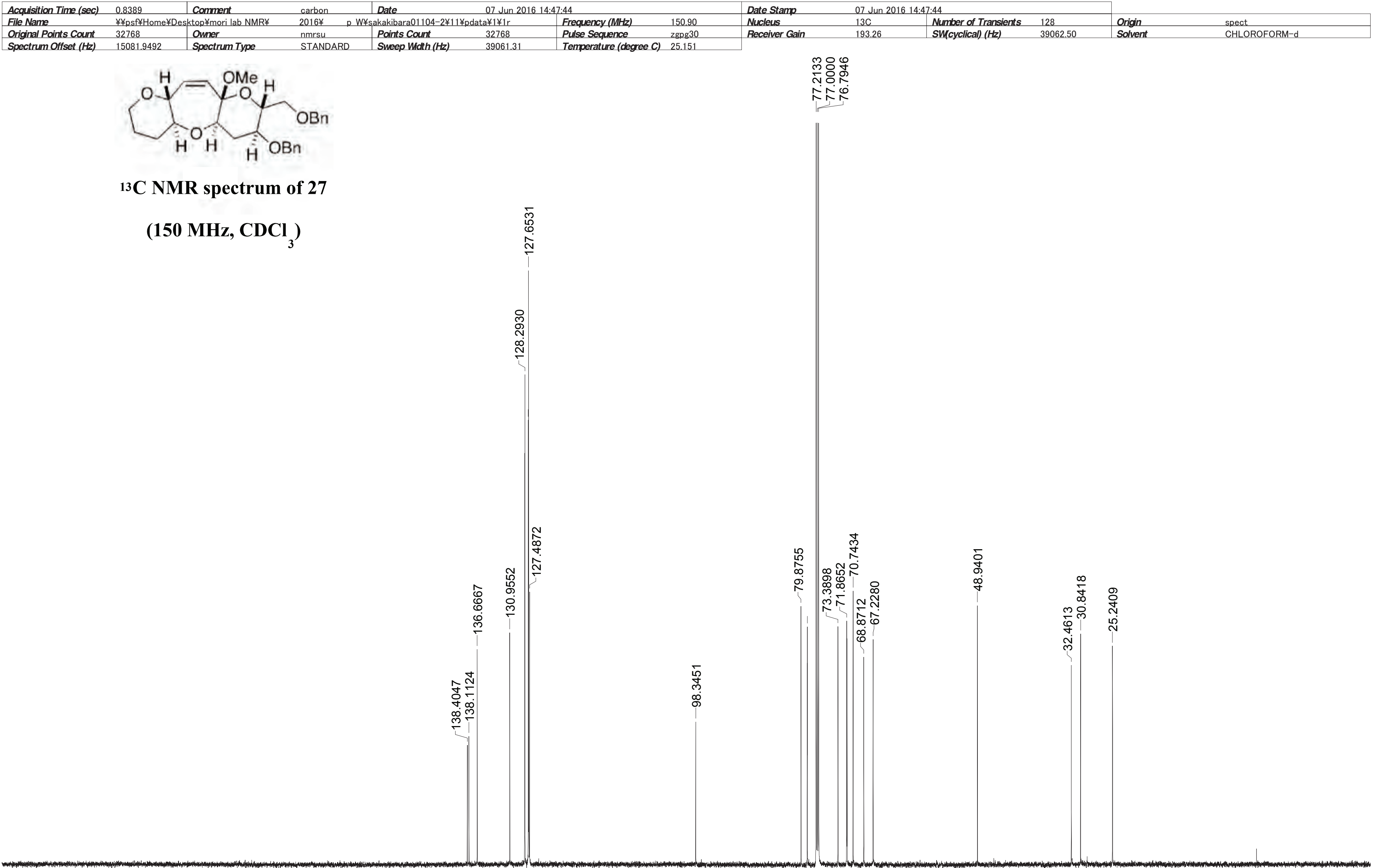


This report was created by ACD/NMR Processor Academic Edition. For more information go to www.acdlabs.com/nmrproc/

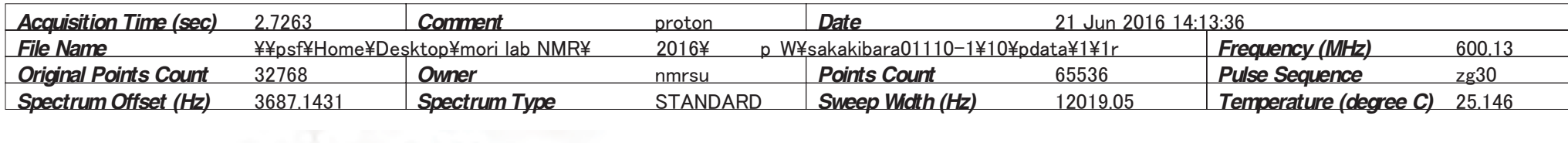

Date Stamp
Nucleus
Res

Rucleus 21 Jun 2016 14:13:36
\begin{tabular}{|l|l|}
\hline 11 & Number of Transients \\
\hline 70.26 & SW(cyclical) (HZ) \\
\hline
\end{tabular}

Origin
Solvent

spect
CHLOROFORM-d

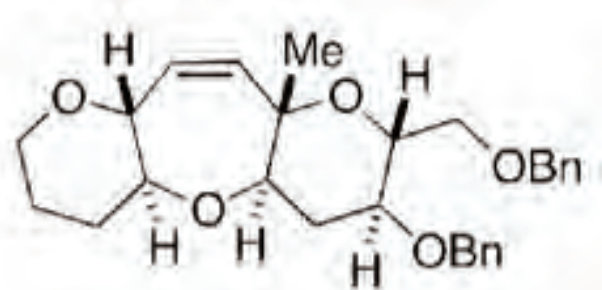

1 H NMR spectrum of 29

(600 $\left.\mathrm{MHz}_{,} \mathrm{CDCl}_{3}\right)$ 
This report was created by ACD/NMR Processor Academic Edition. For more information go to www.acdlabs.com/nmrproc/

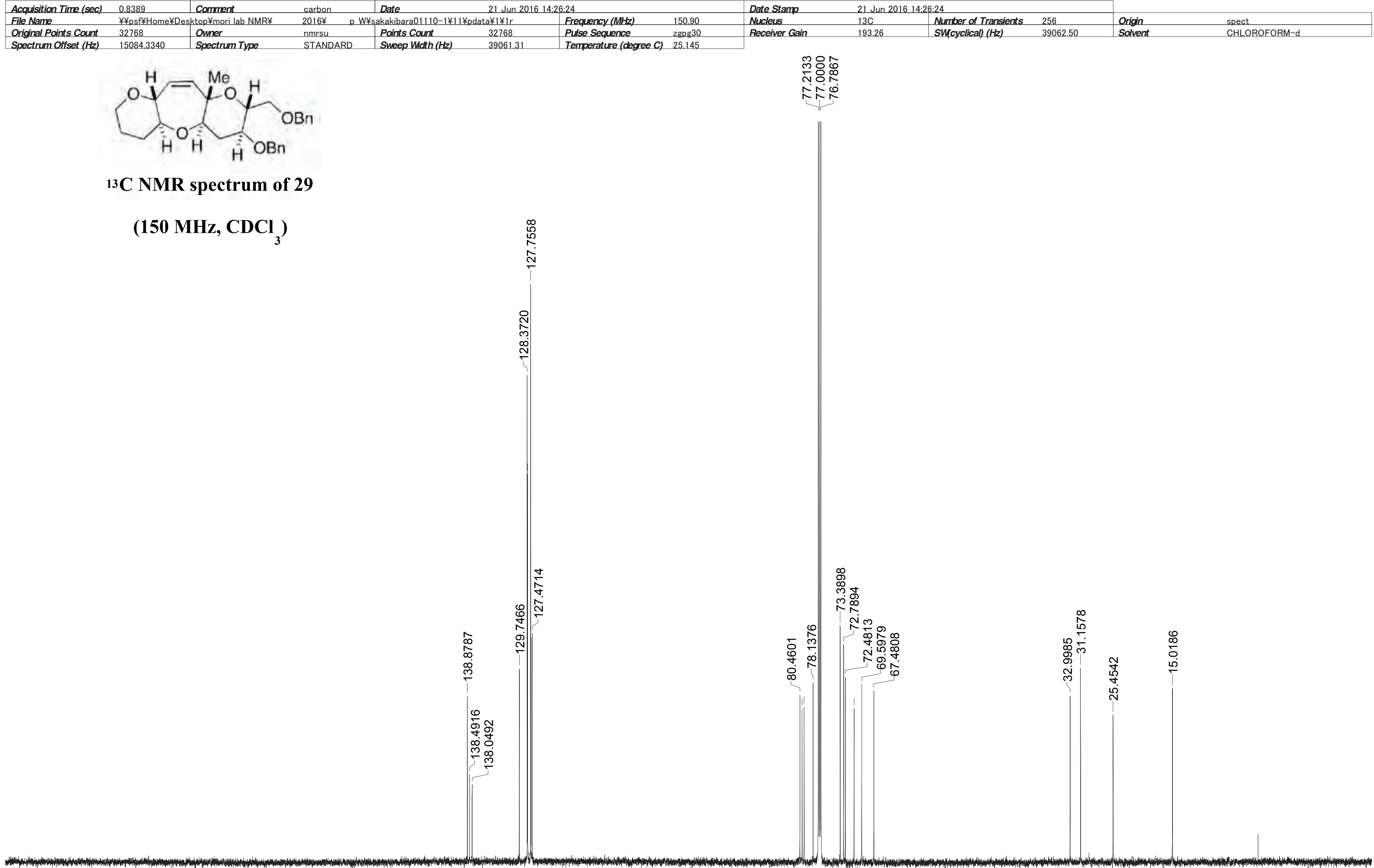


This report was created by ACD/NMR Processor Academic Edition. For more information go to www.acdlabs.com/nmrproc/

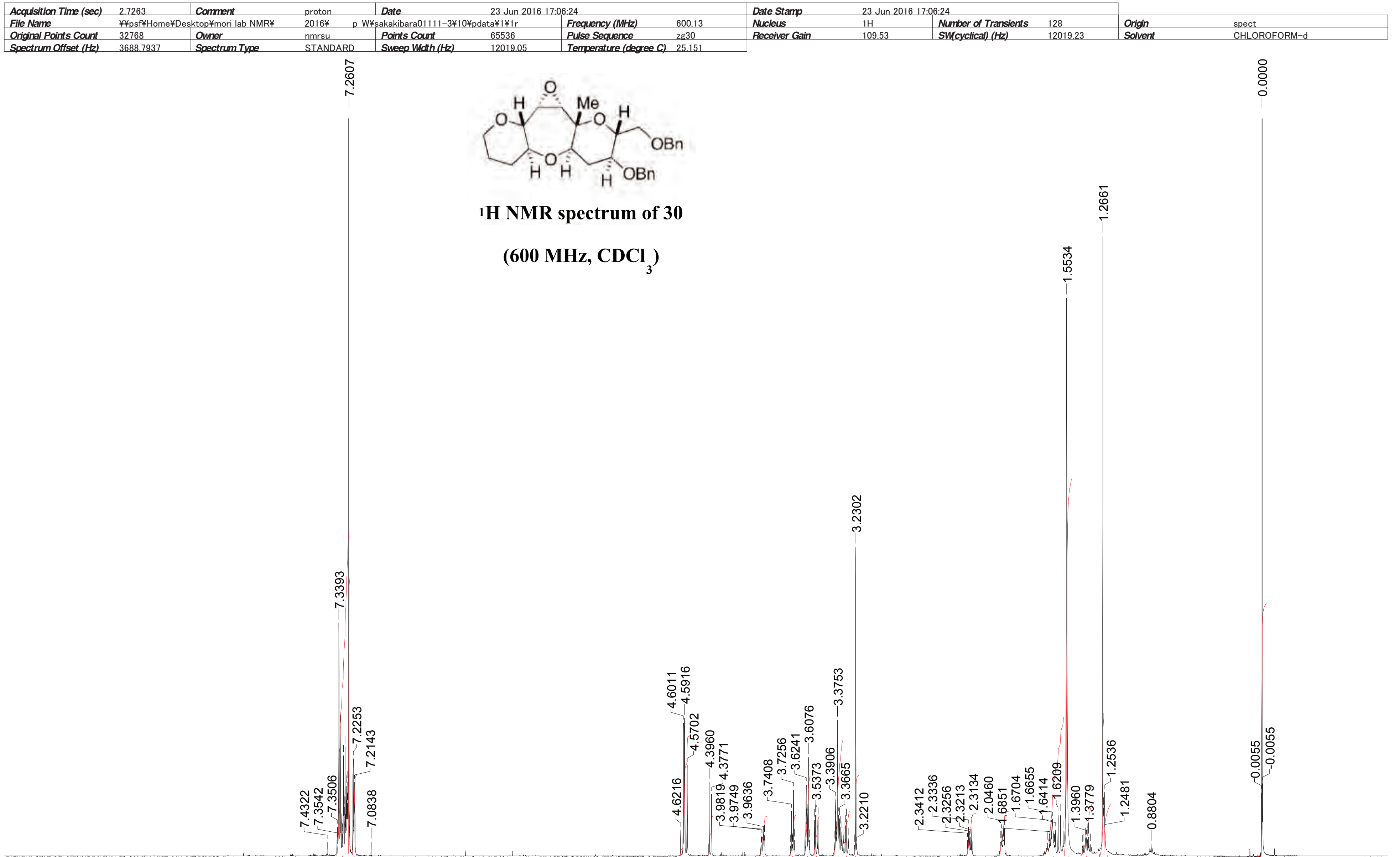

8.046 .962 .02

$2.991 .00 \quad 0.990 .001 .021 .990 .992 .931 .122 .01$

$1.06 \quad 1.133 .529 .401 .310 .073 .611 .29$ 
This report was created by ACD/NMR Processor Academic Edition. For more information go to www.acdlabs.com/nmrproc/

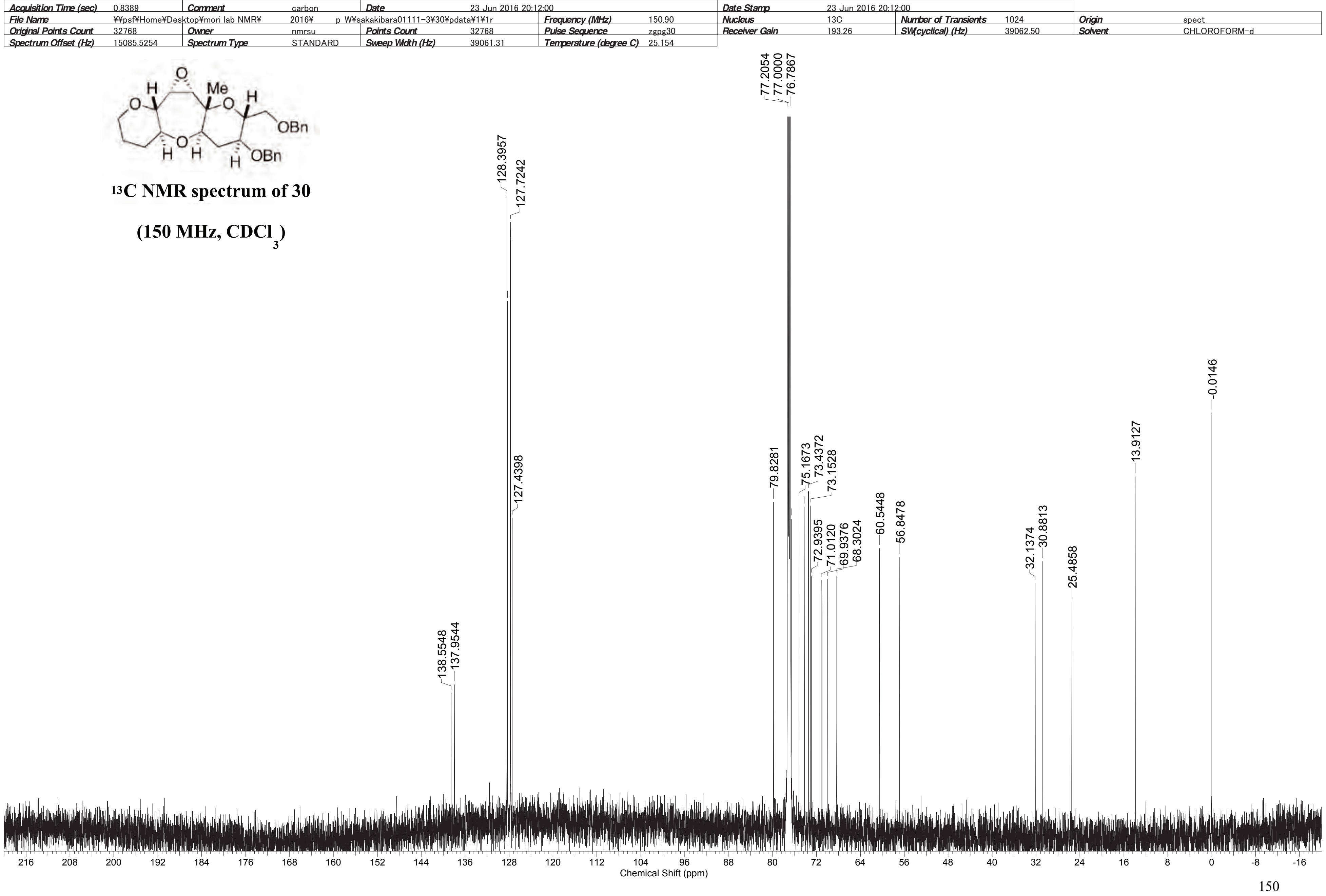


This report was created by ACD/NMR Processor Academic Edition. For more information go to www.acdlabs.com/nmrproc/

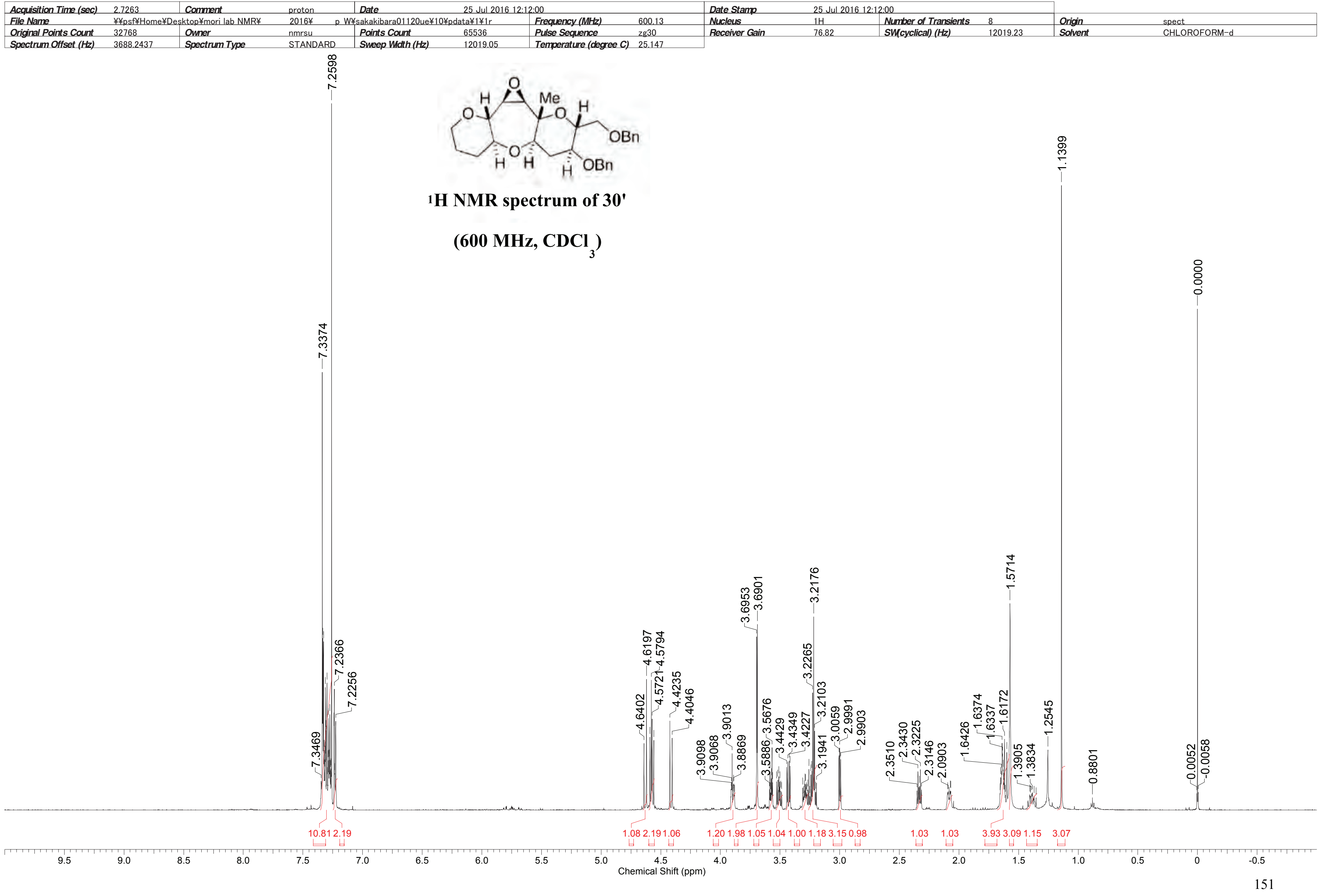


This report was created by ACD/NMR Processor Academic Edition. For more information go to www.acdlabs.com/nmrproc/

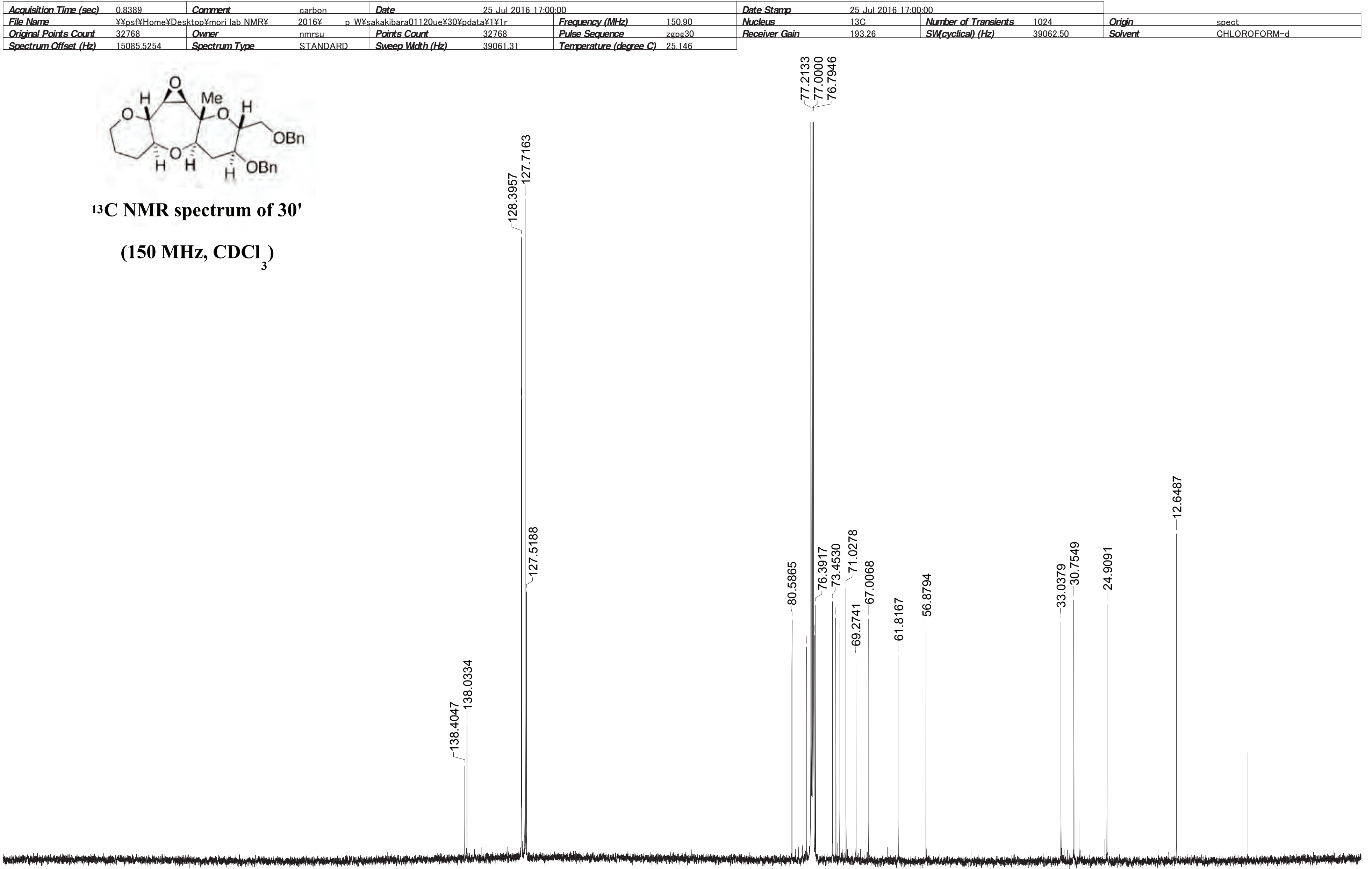


This report was created by ACD/NMR Processor Academic Edition. For more information go to www.acdlabs.com/nmrproc/

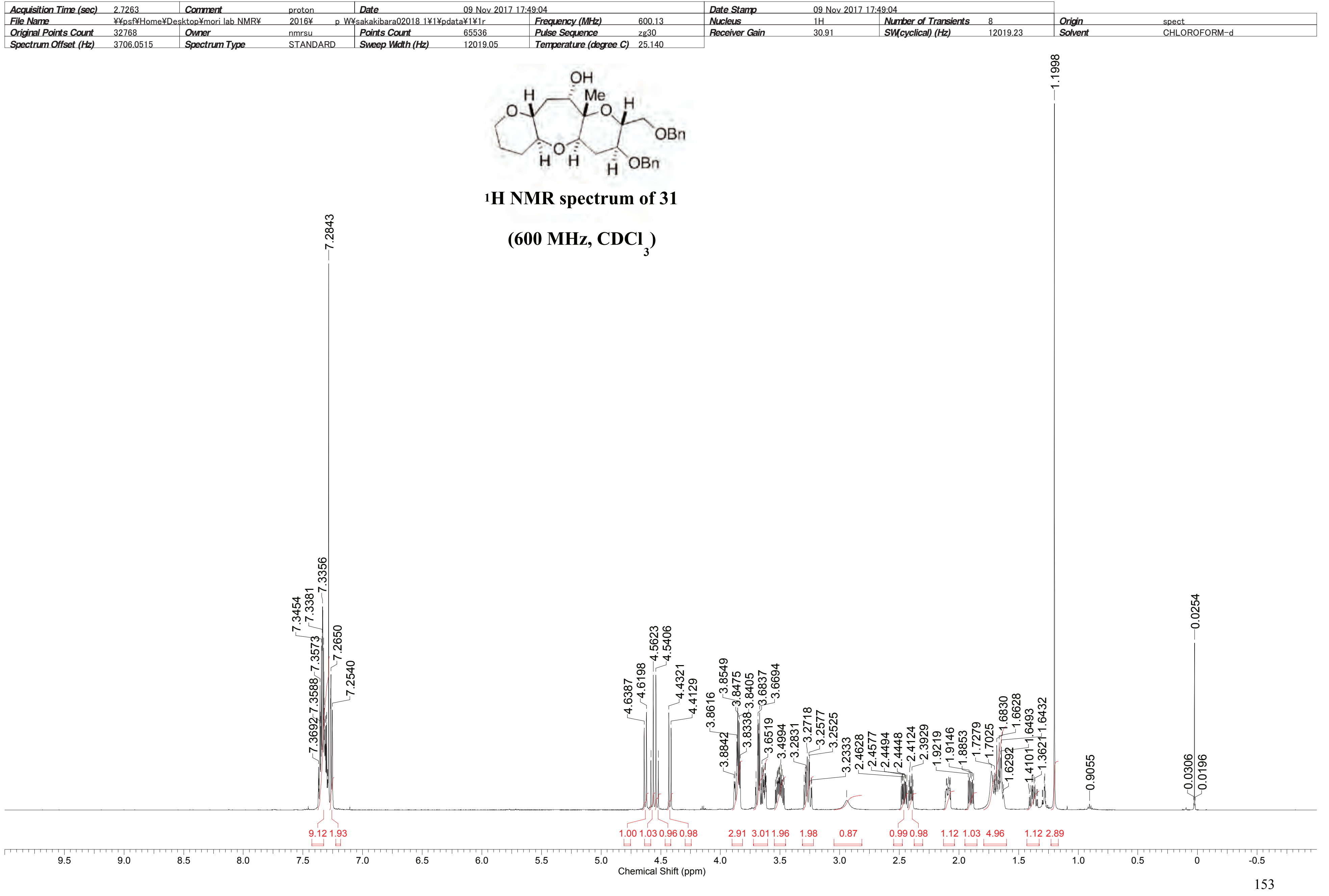


This report was created by ACD/NMR Processor Academic Edition. For more information go to www.acdlabs.com/nmrproc/

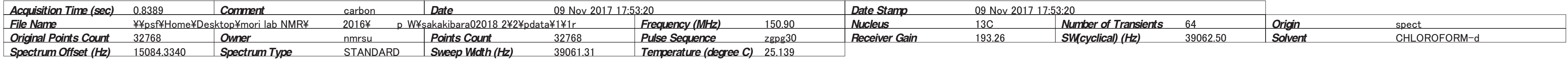

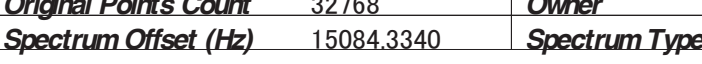
\begin{tabular}{l|l} 
& Points Count \\
\hline
\end{tabular} zeggen Nacleus m.

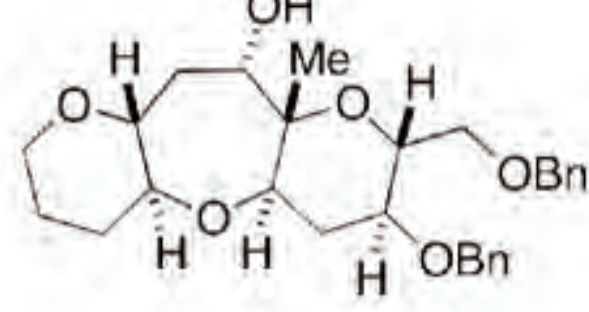

13C NMR spectrum of 31

(150 $\left.\mathrm{MHz} \mathrm{CDCl}_{3}\right)$

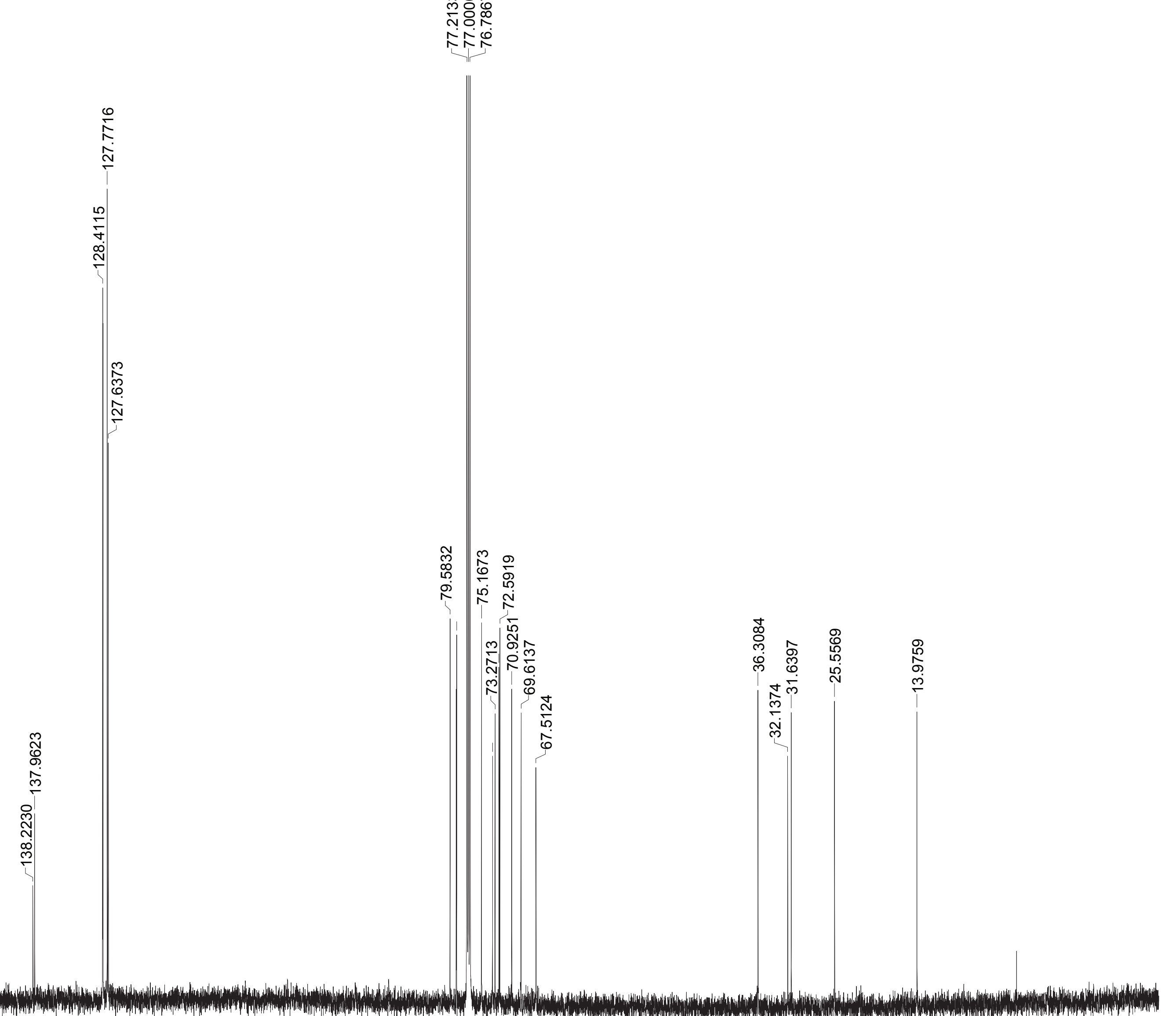

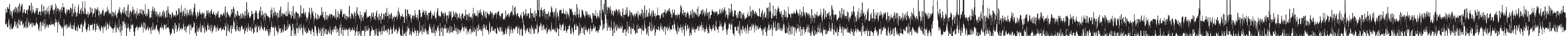


This report was created by ACD/NMR Processor Academic Edition. For more information go to www.acdlabs.com/nmrproc/

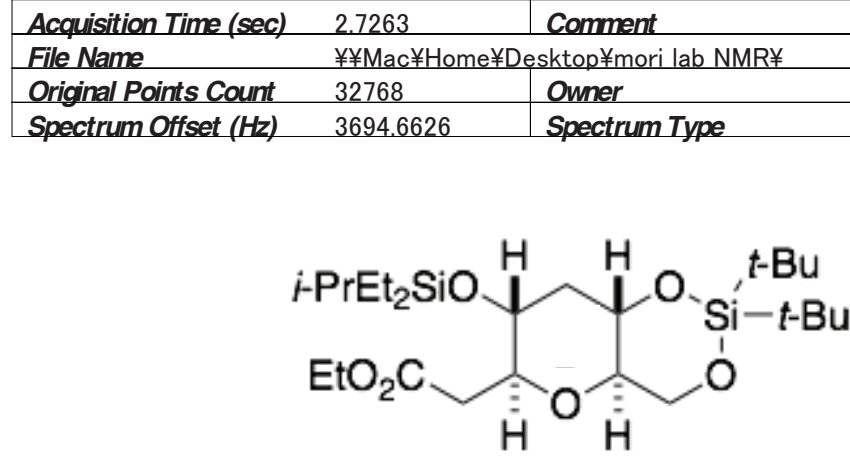

1H NMR spectrum of S16

(600 MHz, $\left.\mathrm{CDCl}_{3}\right)$.

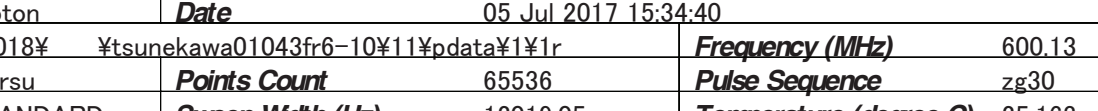

Date Stamp Receiver Gain Number of Transients
SW(cyclical) $(\mathrm{Hz})$

16

Origin

CHLOROFORM-d

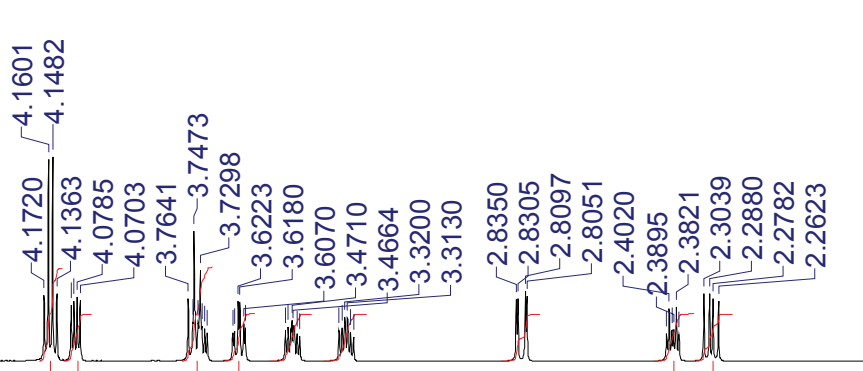

$1.991 .00 \quad 2.011 .011 .011 .00$
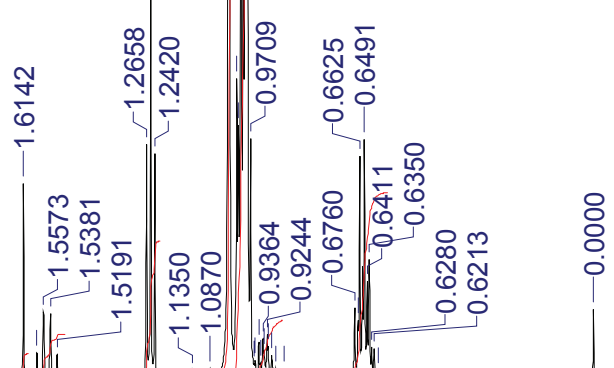
This report was created by ACD/NMR Processor Academic Edition. For more information go to www.acdlabs.com/nmrproc/

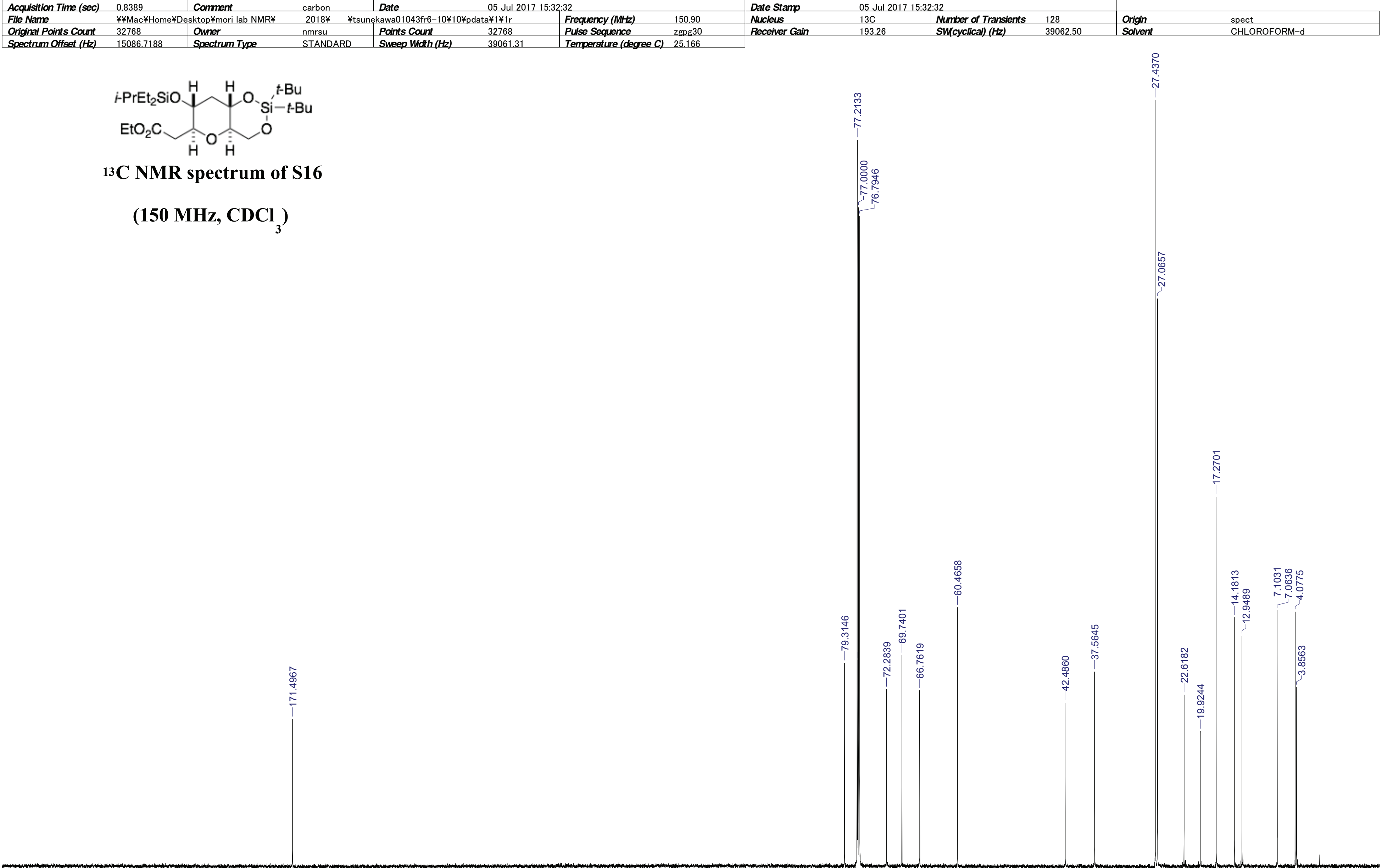


This report was created by ACD/NMR Processor Academic Edition. For more information go to www.acdlabs.com/nmrproc/

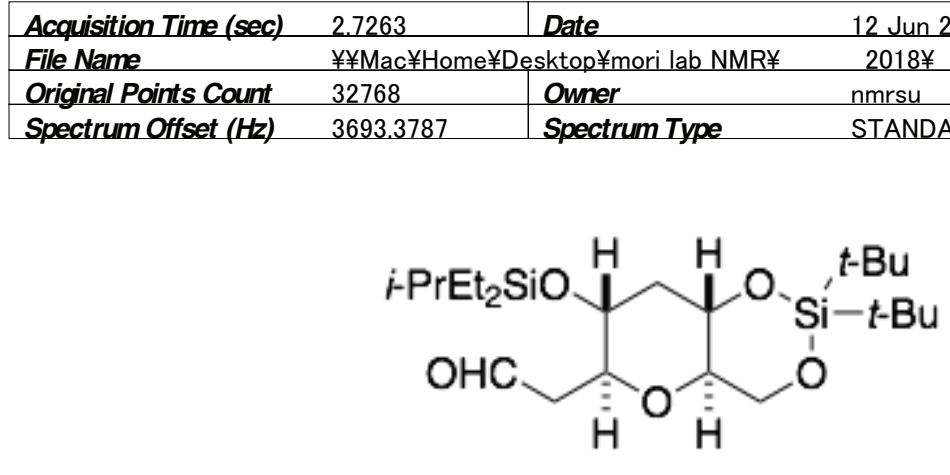

Date Stamp $\quad 12$ Jun 2019 18:25:20

Rucleus

Number of Transients
Sw(yclical) $(H z)$ $\begin{array}{ll}\text { Pulse Sequence } & \text { zg30 } \\ \text { Temperature (degree C) } & 25.151\end{array}$

Origin CHLOROFORM-d

1H NMR spectrum of S17

(600 MHz, CDCl )

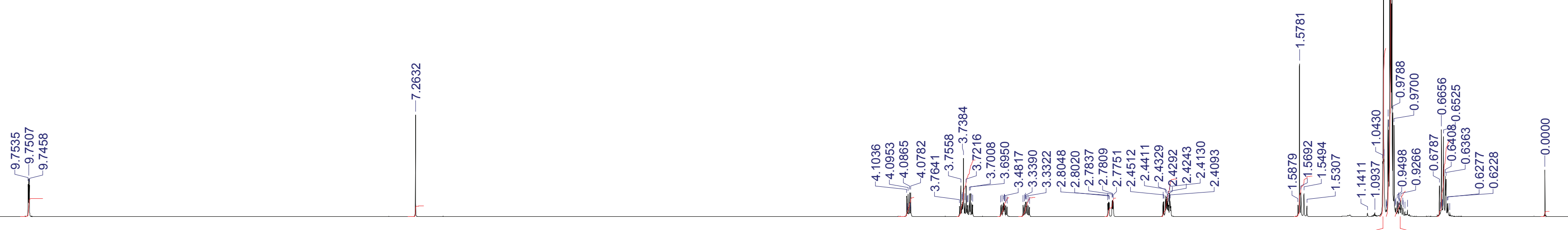


This report was created by ACD/NMR Processor Academic Edition. For more information go to www.acdlabs.com/nmrproc/

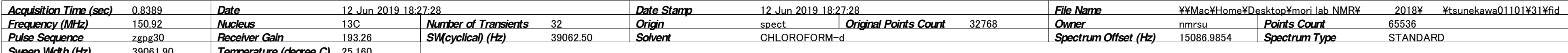

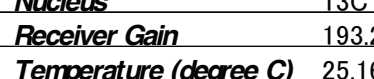
\begin{tabular}{lll} 
Number of Transients & 32 & Origin \\
\hline SW(cyclical) (Hz) & 39062.50 & Solve
\end{tabular} 15086.9854 Pectrum Type

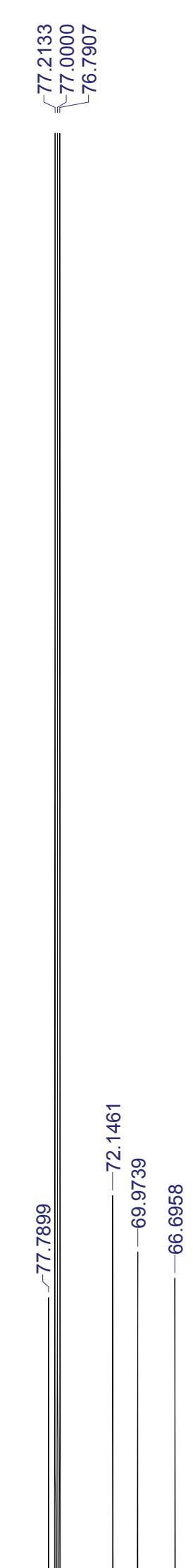

(150 MHz, CDCl )

$$
\begin{aligned}
& \text { - } \mathrm{PrEt}_{2} \mathrm{SiO} \underbrace{\mathrm{H} O} \mathrm{Si}^{t} \mathrm{-Bu} \\
& \mathrm{OHC} \curvearrowright \mathrm{O}
\end{aligned}
$$

\section{C NMR spectrum of S17}

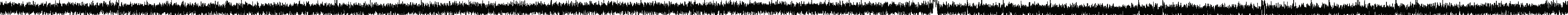


This report was created by ACD/NMR Processor Academic Edition. For more information go to www.acdlabs.com/nmrproc/

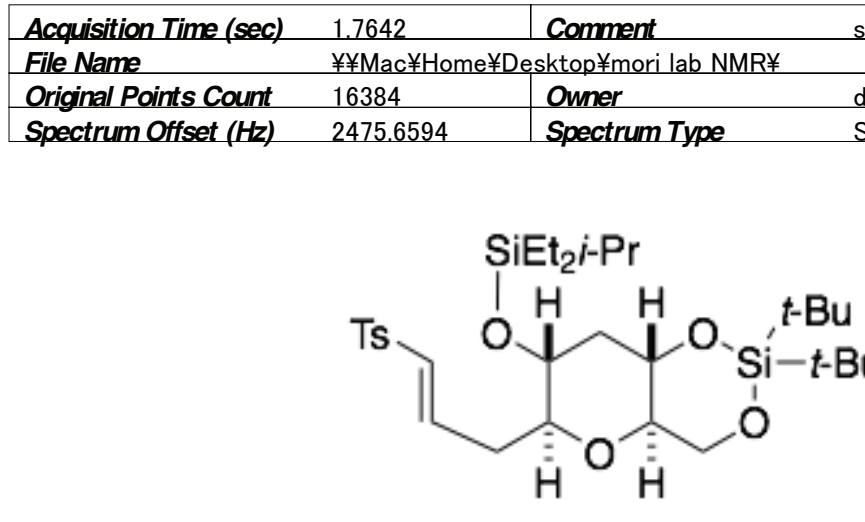

1H NMR spectrum of S18

$\left(500 \mathrm{MHz}_{2} \mathrm{CDCl}_{3}\right)$

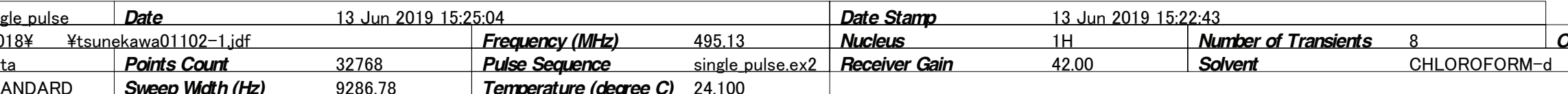

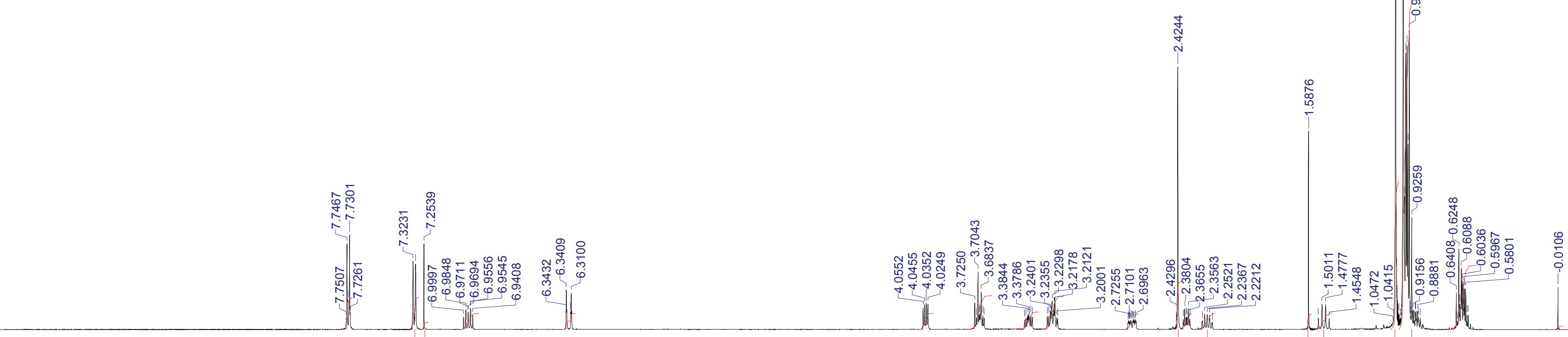

$1.97 \quad 2.000 .44 \quad 1.00 \quad 1.00$

$1.01 \quad 2.11 \quad 1.102 .07 \quad 0.982 .991 .001 .00$

$0.951 .03 \quad 9.2422 .16 \quad 4.00$ 
This report was created by ACD/NMR Processor Academic Edition. For more information go to www.acdlabs.com/nmrproc/

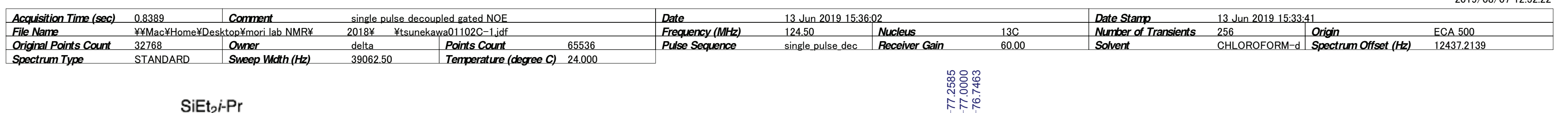

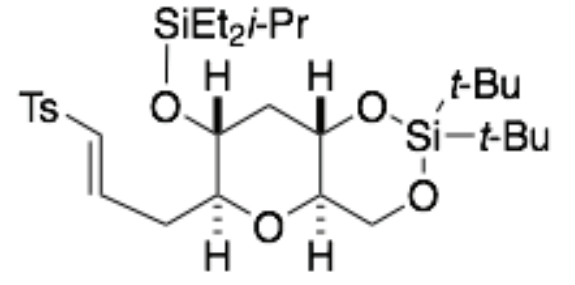

${ }_{13 \mathrm{C}}$ NMR spectrum of S18

(125 MHz, $\mathrm{CDCl}_{3}$ )

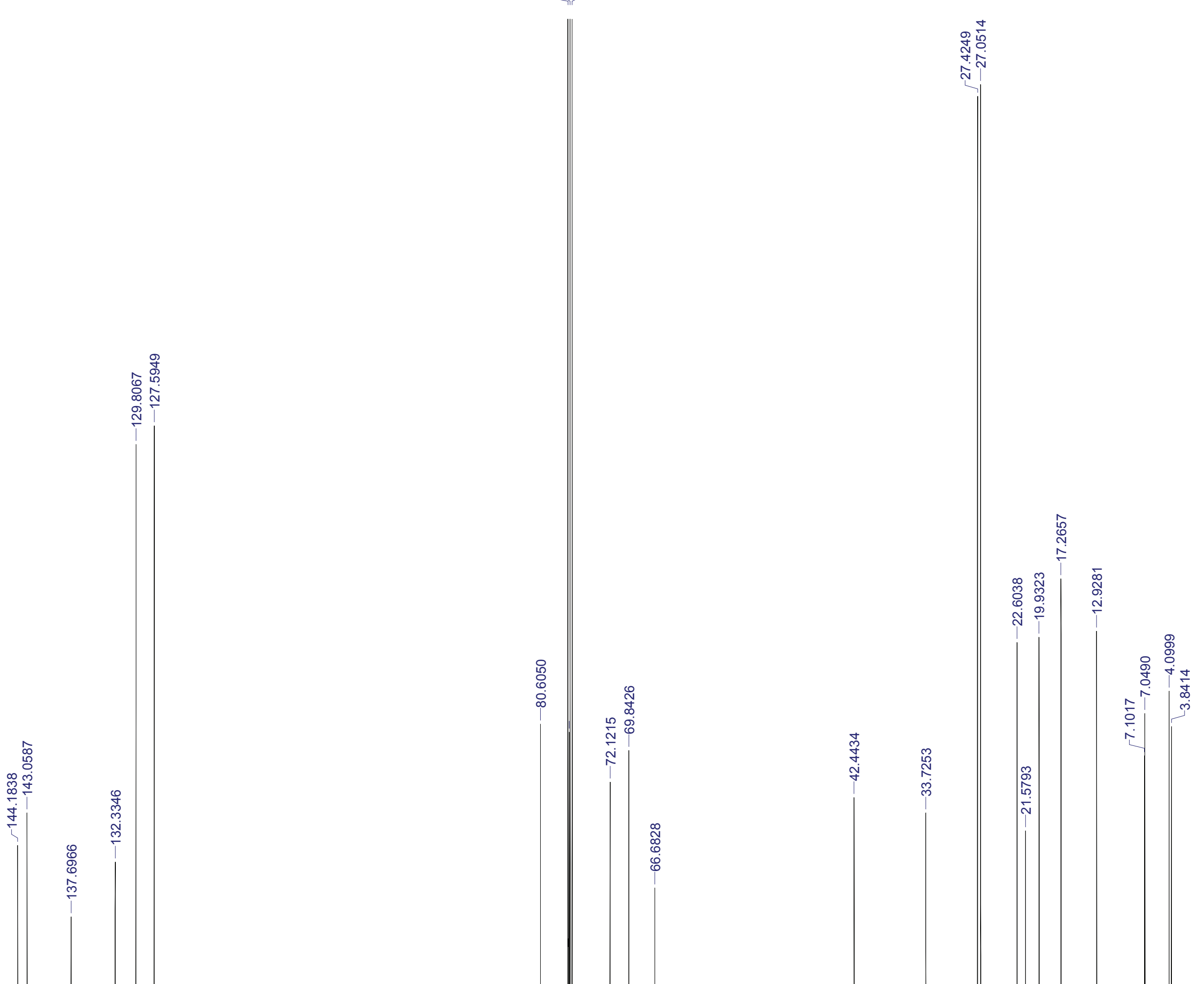

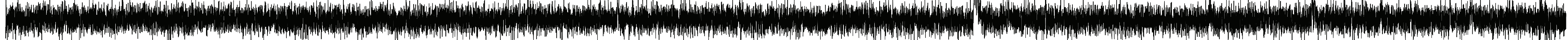

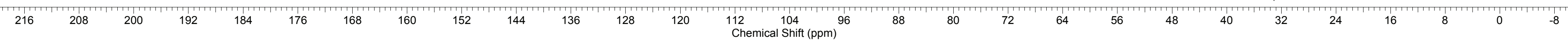


This report was created by ACD/NMR Processor Academic Edition. For more information go to www.acdlabs.com/nmrproc/

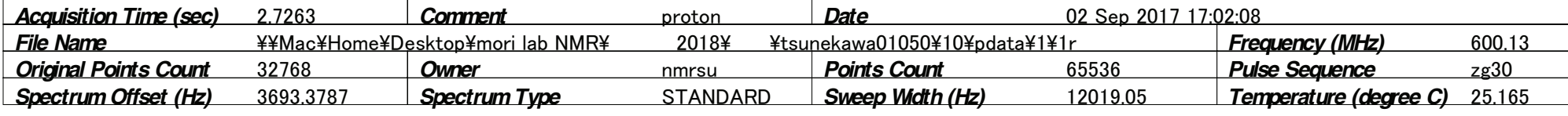
Date Stamp
Nucleus
Receiver Gain 02 Sep 2017 17:02:08

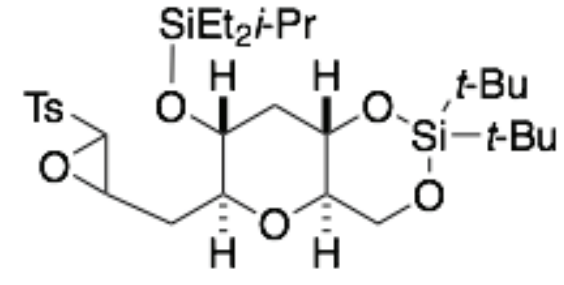

$(\mathrm{dr}=85: 15)$

1 H NMR spectrum of 3

(600 MHz, CDCl $\left.{ }_{3}\right)$

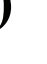




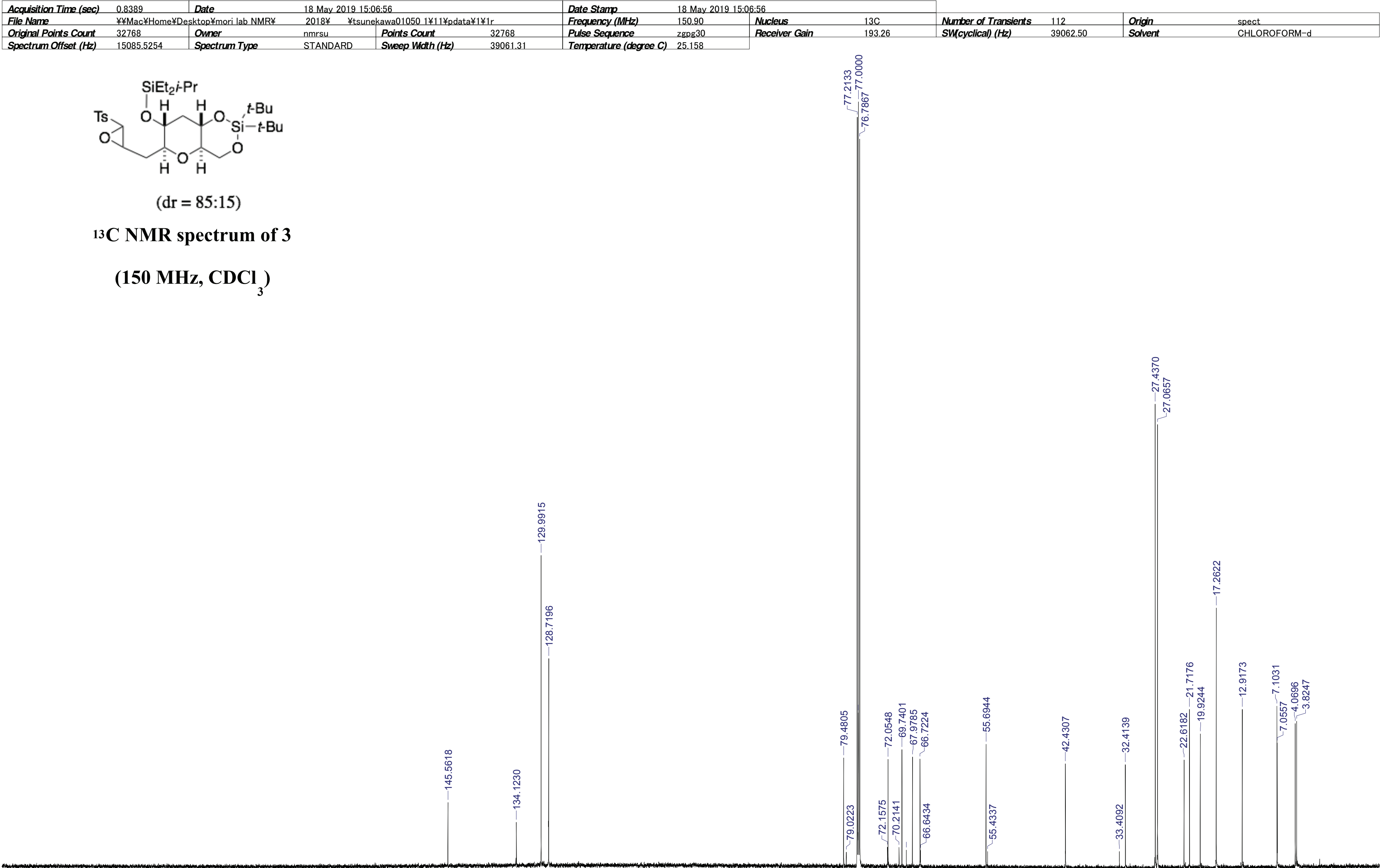




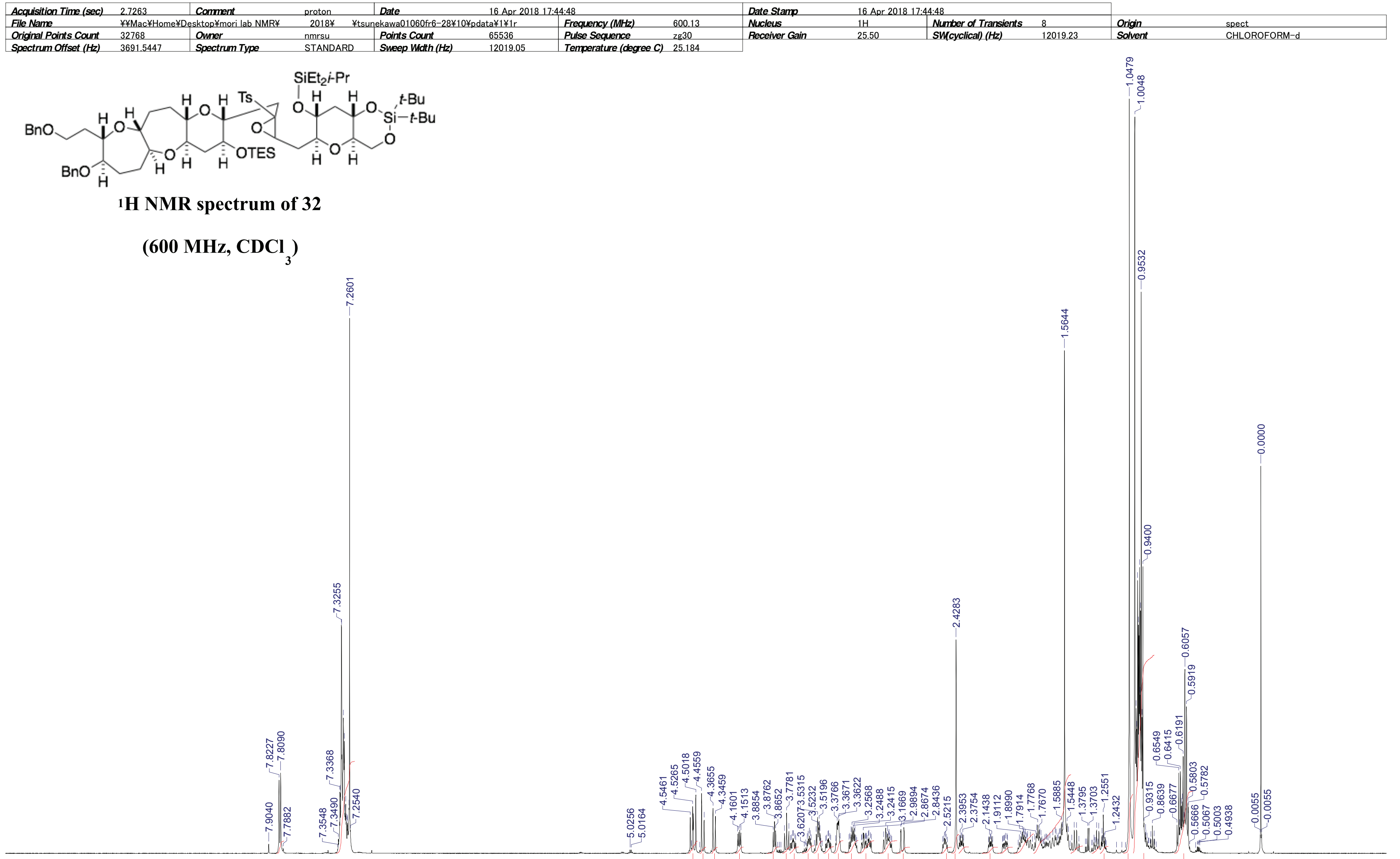

$1.96 \quad 14.71$

1.96 0.970 .951 .001 .041 .061 .021 .211 .981 .062 .032 .062 .002 .000 .961 .003 .031 .040 .990 .983 .1312 .591 .161 .171 .129 .4631 .769 .95 


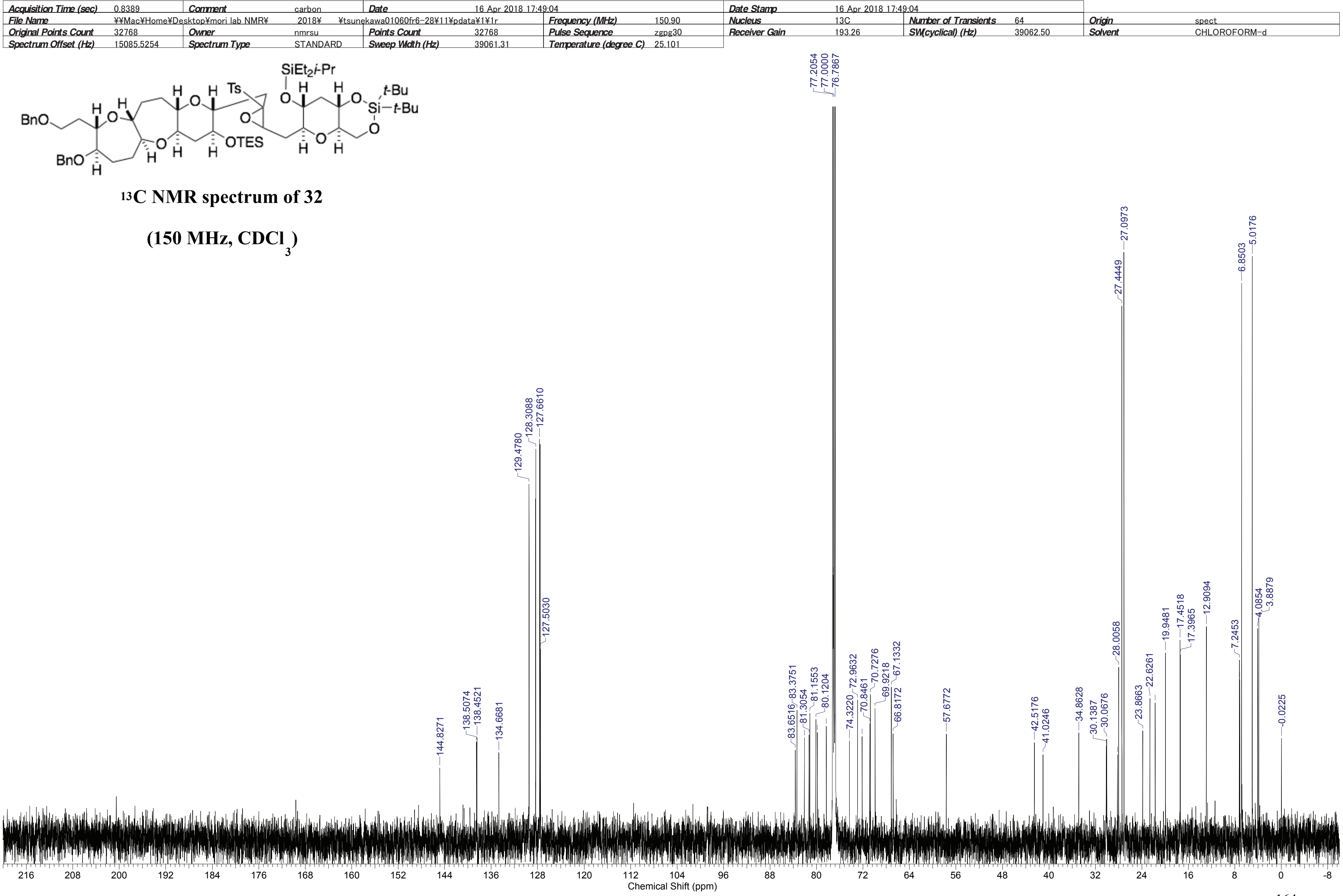


This report was created by ACD/NMR Processor Academic Edition. For more information go to www.acdlabs.com/nmrproc/

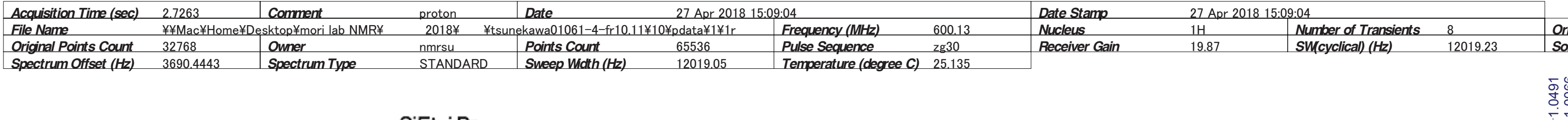

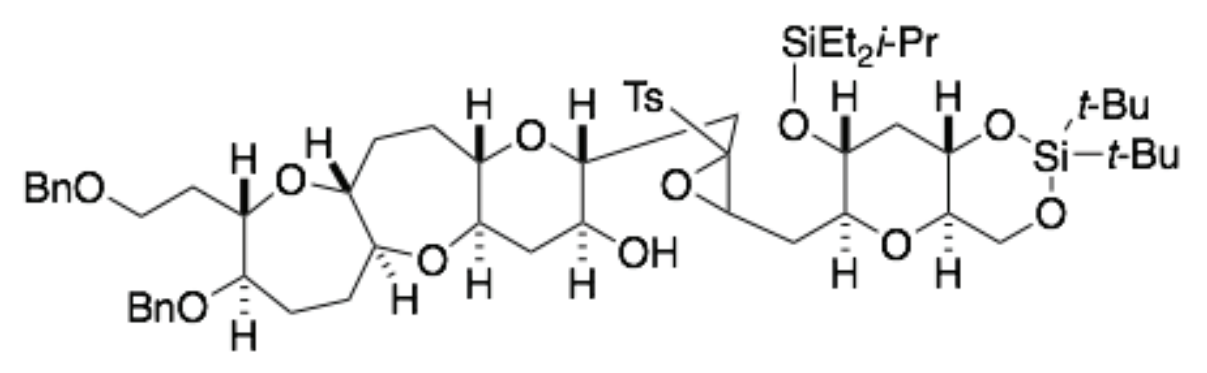

\section{H NMR spectrum of 33}

(600 MHz, $\mathrm{CDCl}_{3}$ )

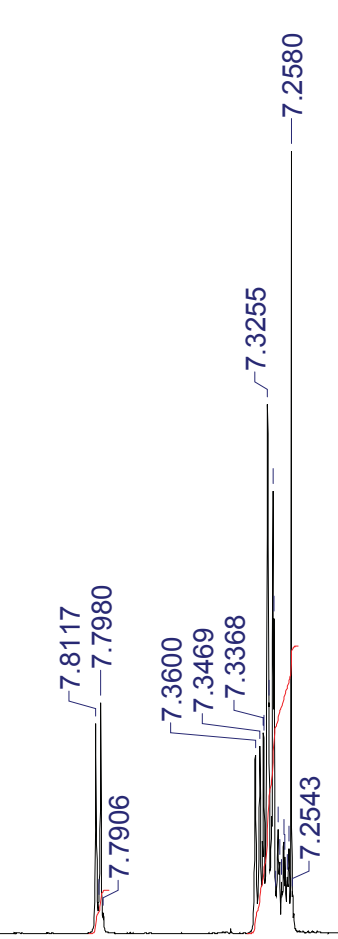

1.04 1.031.07 1.05 1.00 2.06 1.03 1.09 2.123.09 2.17 2.09 1.00 1.030.070.96 0.98 3.16 2.121.87 1.151.88 4.406.38 1.08 1.029.48 8.68 15.26 4.28

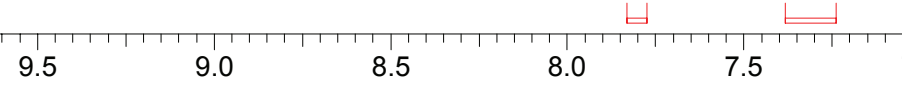


This report was created by ACD/NMR Processor Academic Edition. For more information go to www.acdlabs.com/nmrproc/

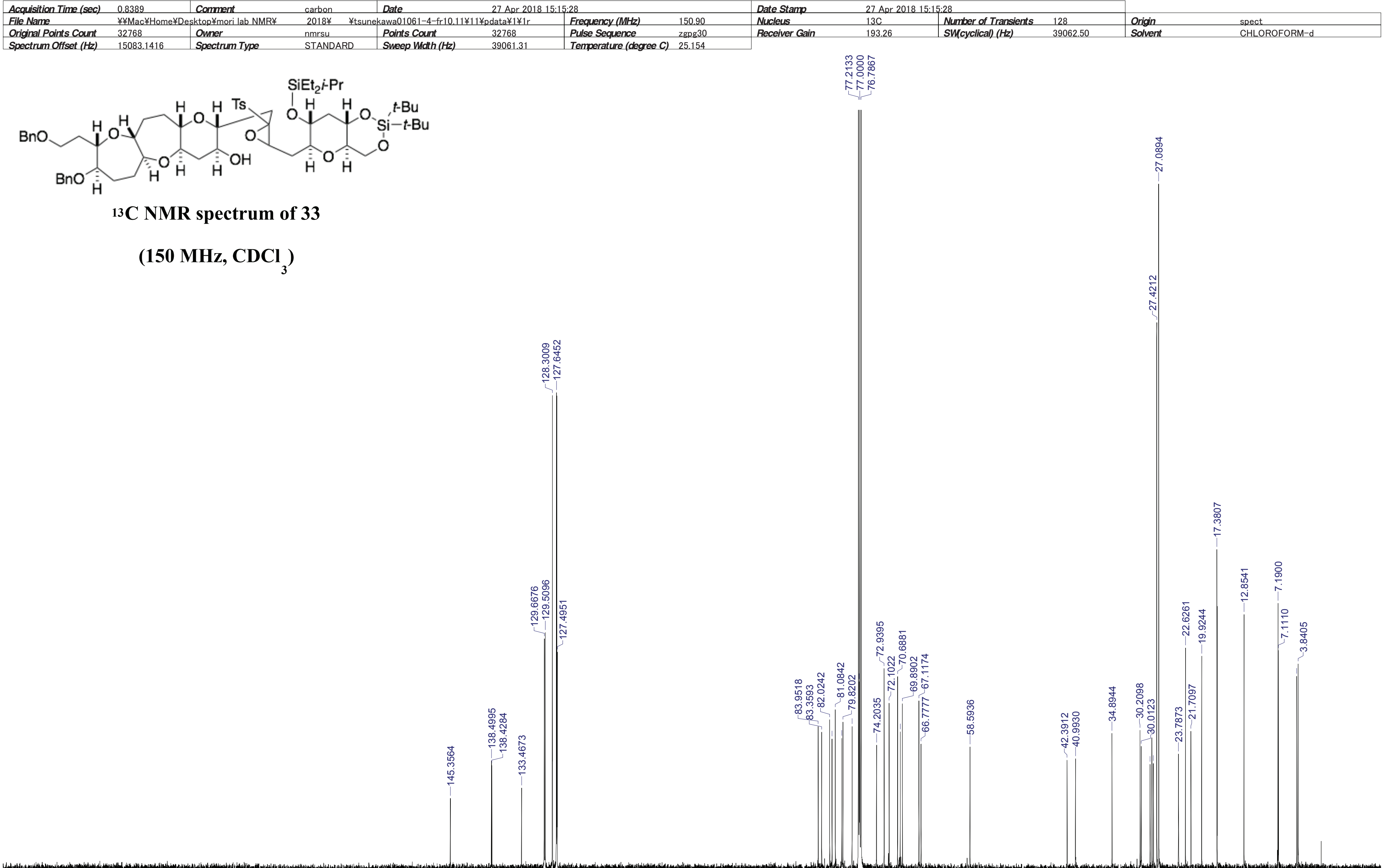


This report was created by ACD/NMR Processor Academic Edition. For more information go to www.acdlabs.com/nmrproc/

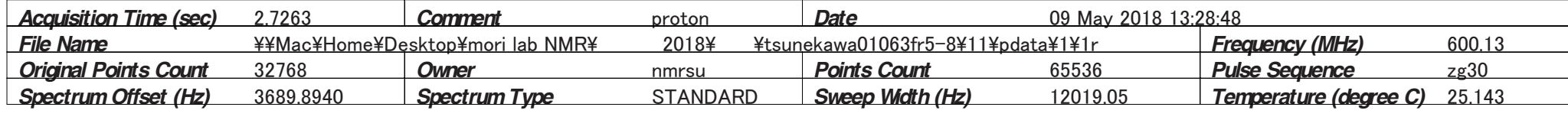

Date Stan

Receiver Gain

09 May 2018 13:28:48

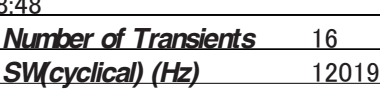

Origin

Solven

spect
CHLOROFORM-d

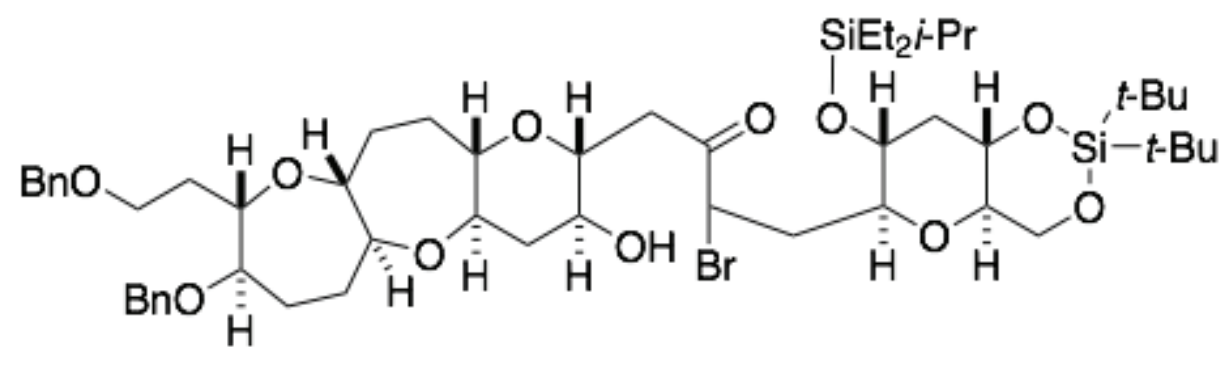

1H NMR spectrum of 34

(600 MHz, CDCl $)_{3}$
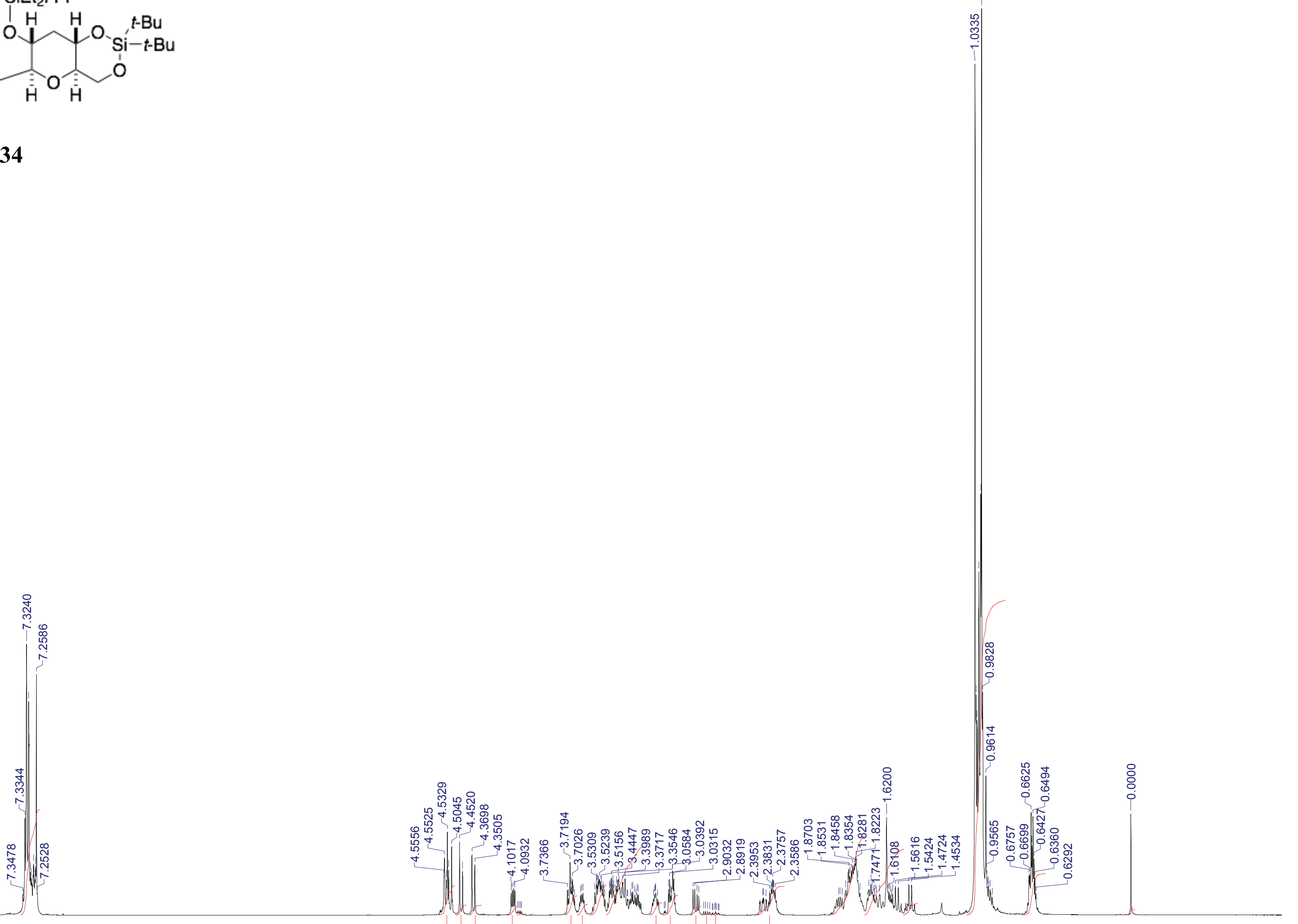

2.951 .010 .970 .840 .151 .951 .063 .026 .831 .241 .980 .840 .130 .122 .89

\begin{tabular}{ll|l}
7.92 & 6.601 .17
\end{tabular} $31.52 \quad 4.17$ 


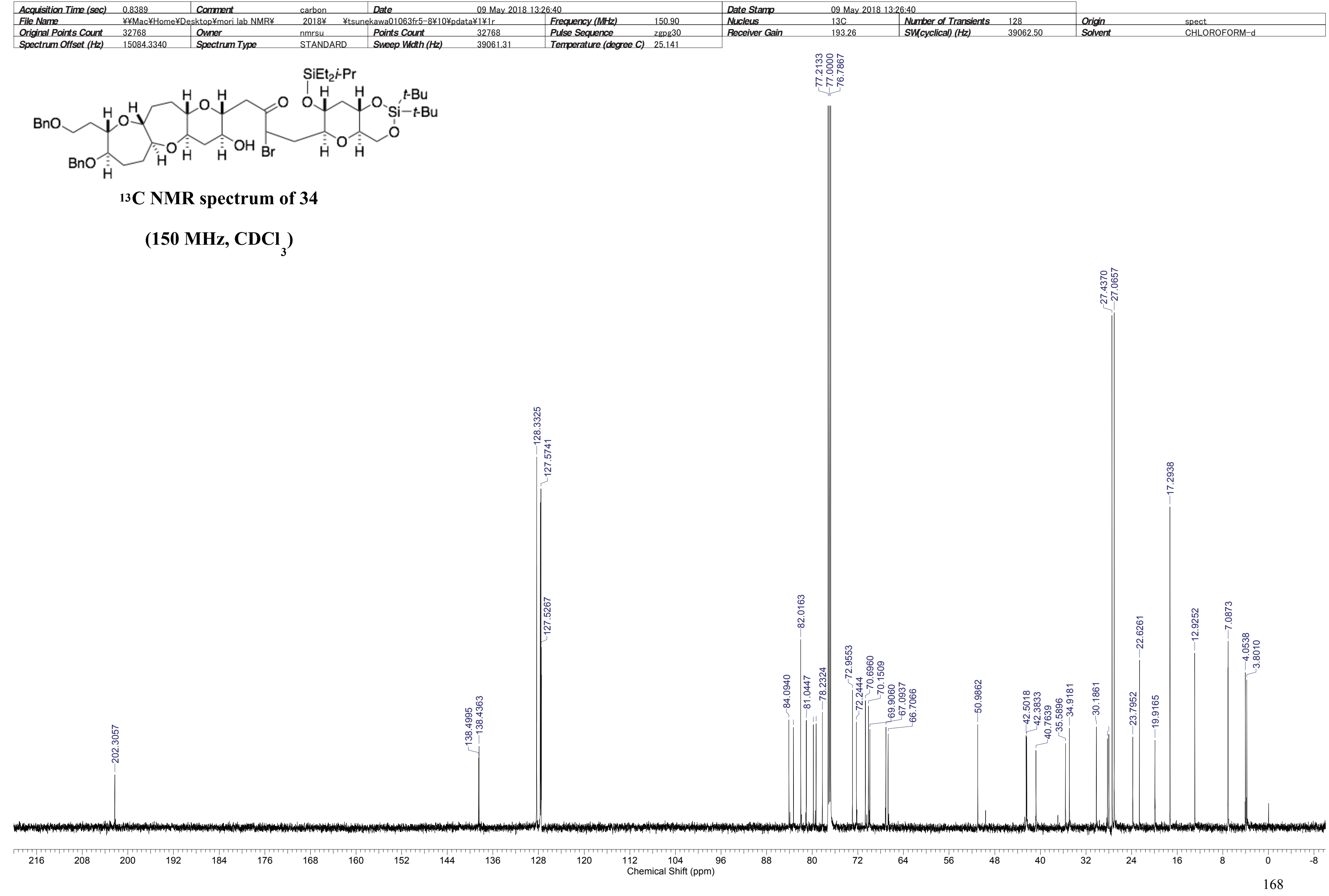


This report was created by ACD/NMR Processor Academic Edition. For more information go to www.acdlabs.com/nmrproc/

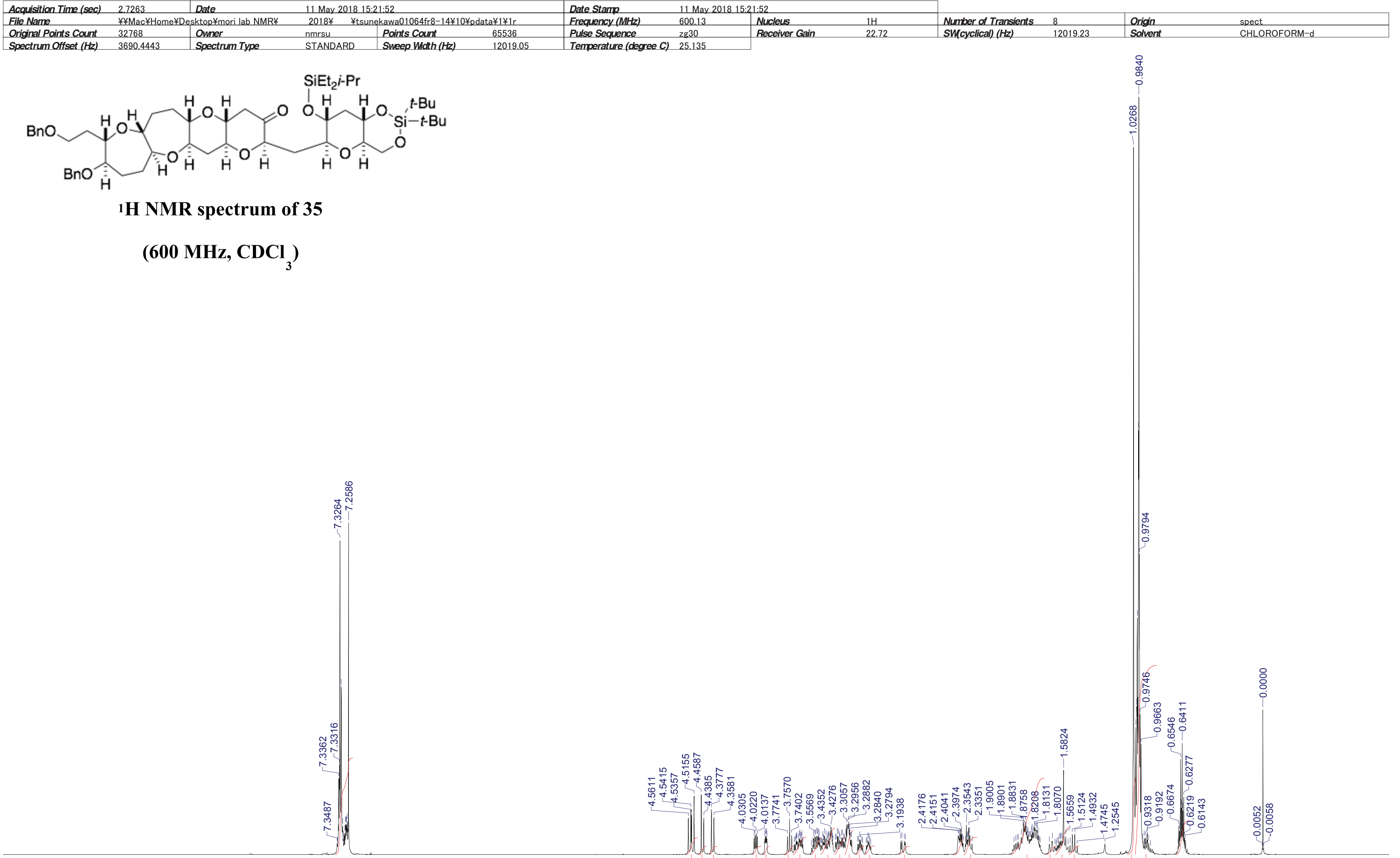

11.37

1.98 1.00 0.97 0.97 0.95 1.00 2.011.93 3.102.152.831.011.01 0.961.98 2.02 $\quad 8.991 .043 .231 .04 \quad 9.0722 .26 \quad 3.99$ 


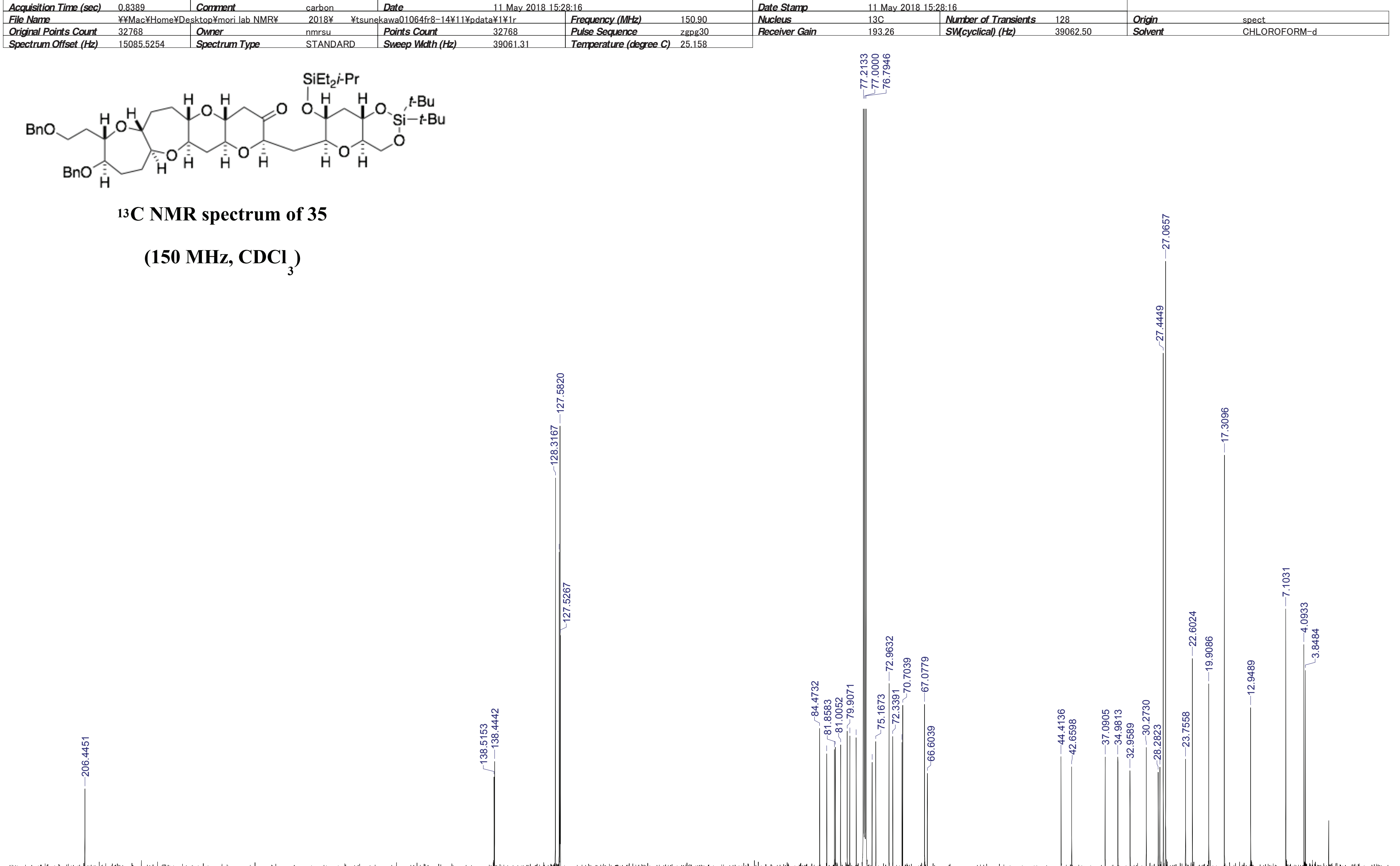




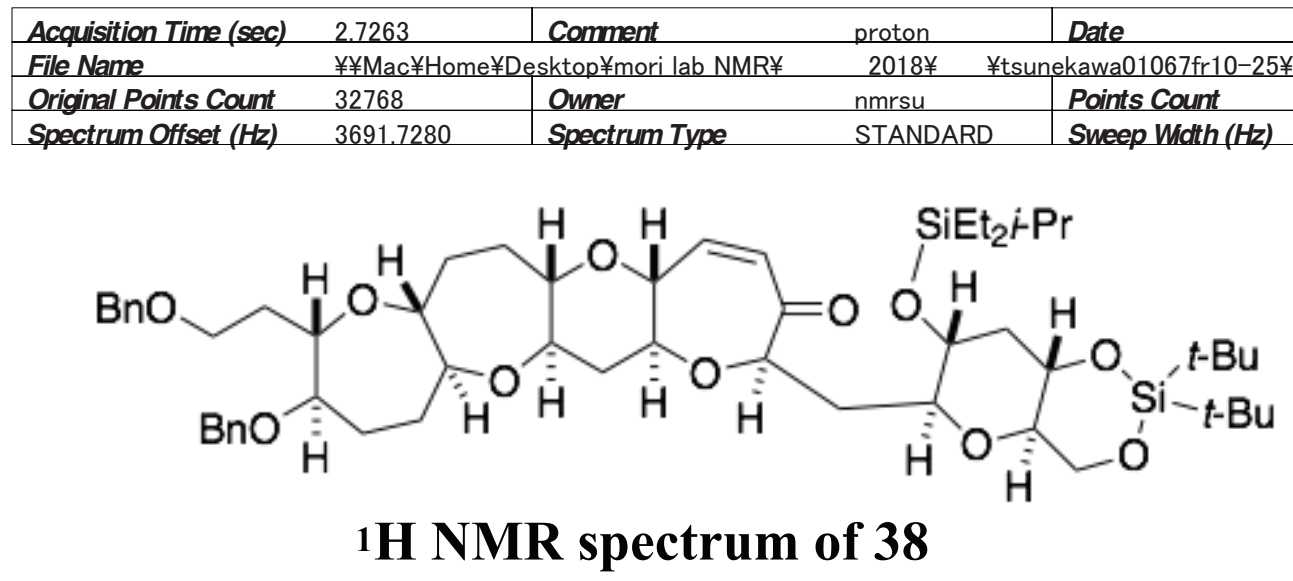

$\left(600 \mathrm{MHz} \mathrm{CDCl}_{3}\right)$

$\begin{array}{llll}\text { Date Stamp } & 23 \text { May 2018 14:24:16 } & \\ \text { Nucleus } & 1 \mathrm{H} & \text { Number of Transients } & 8\end{array}$ Number of Transients
SW(yyclical) $(\mathrm{Hz})$

Frequency (MHz) Pulse Sequence $\quad$ zg30 Receiver Gain $1 \mathrm{H}$
30.91 $\begin{array}{ll}12019.23 & \text { Origin } \\ \text { Solvent }\end{array}$

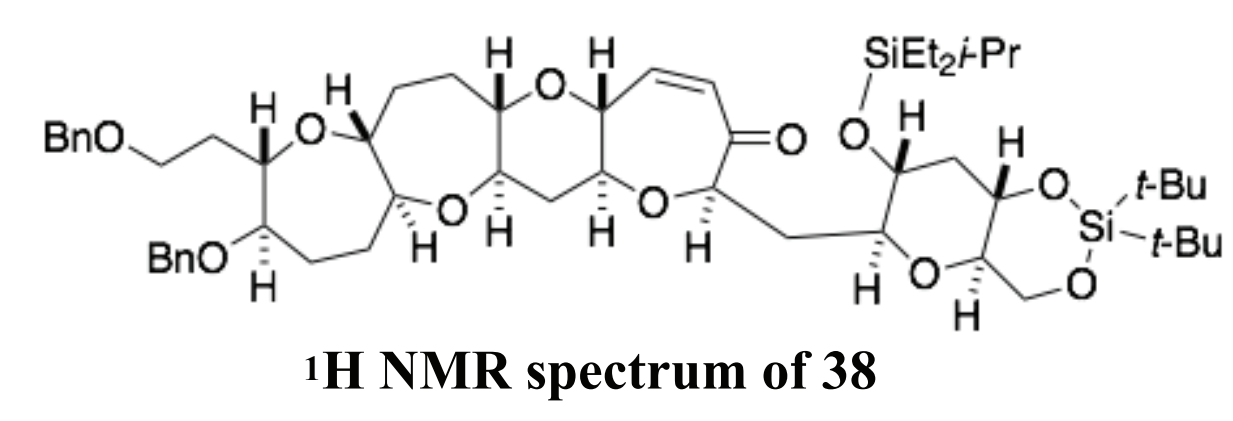
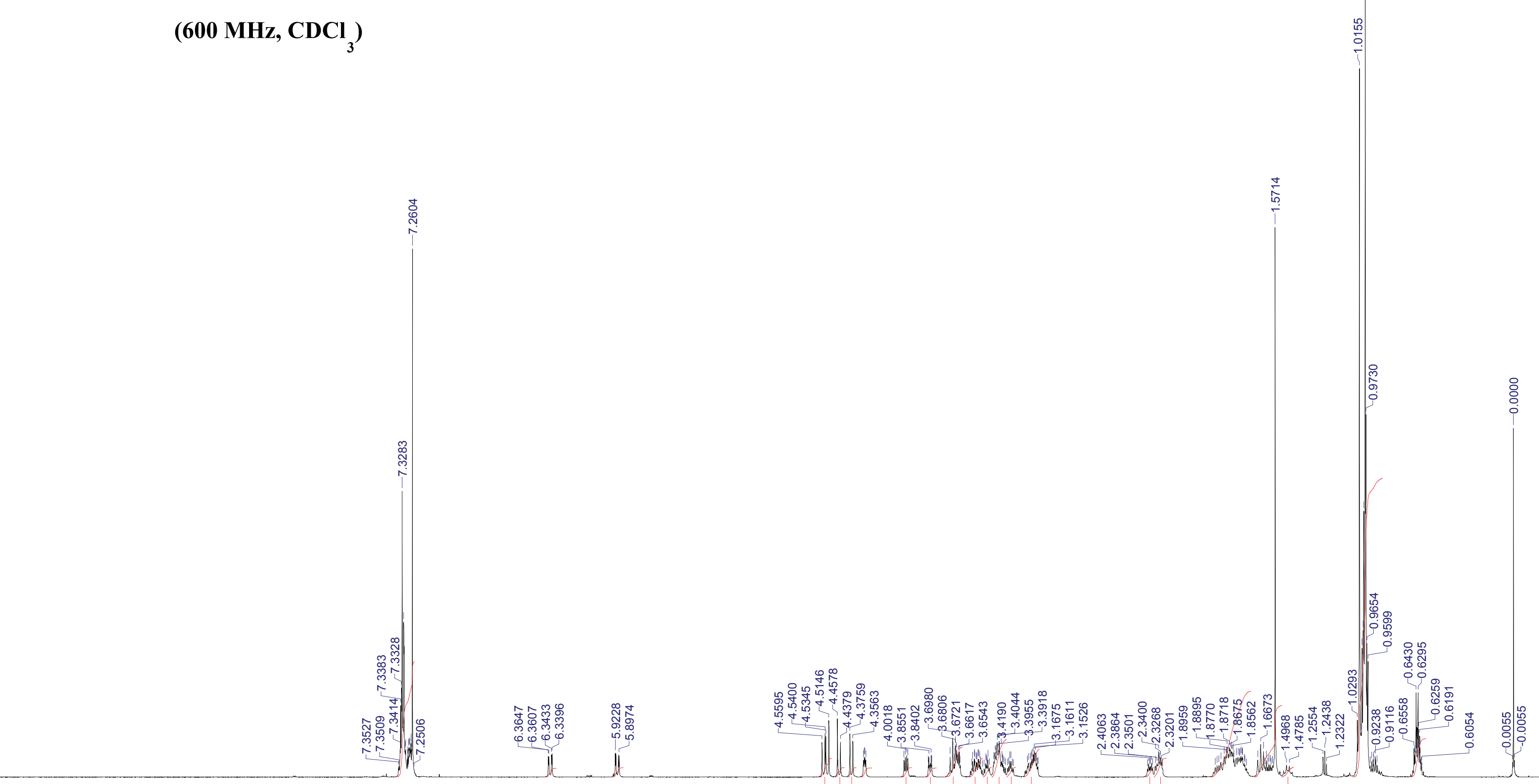

(O)

1.99 1.01 1.000.96 1.010.96 3.192.031.06 4.011.042.94

1.022 .01

9.117 .601 .04

$31.73 \quad 4.08$ 


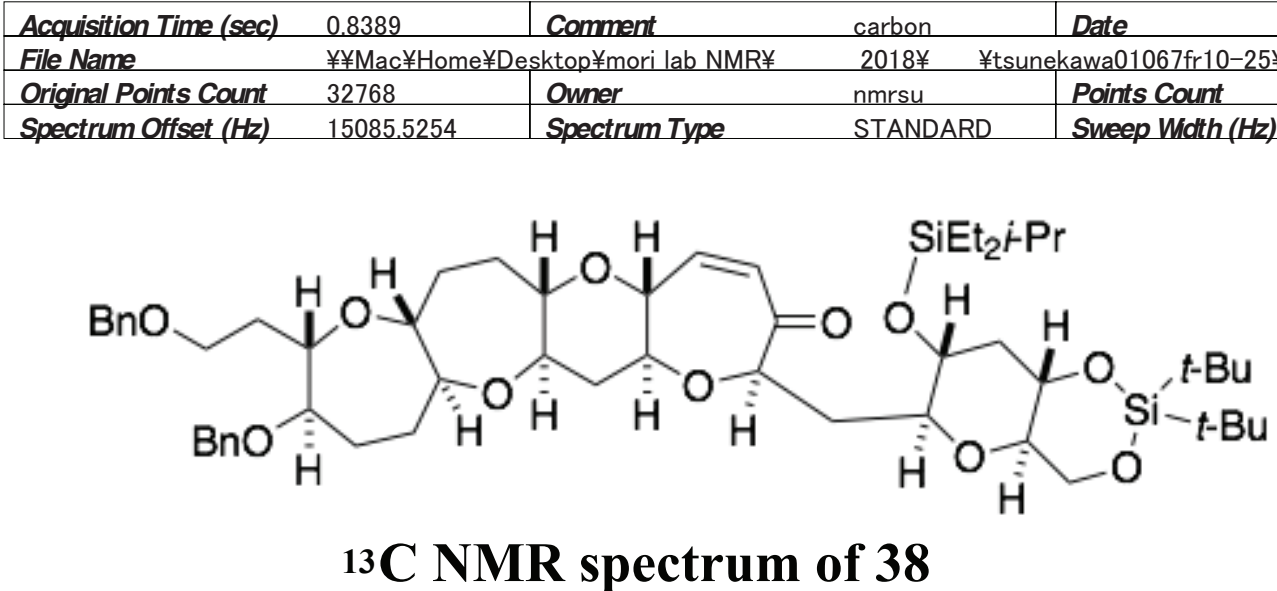

(150 $\mathrm{MHz} \mathrm{CDCl}_{3}$ )

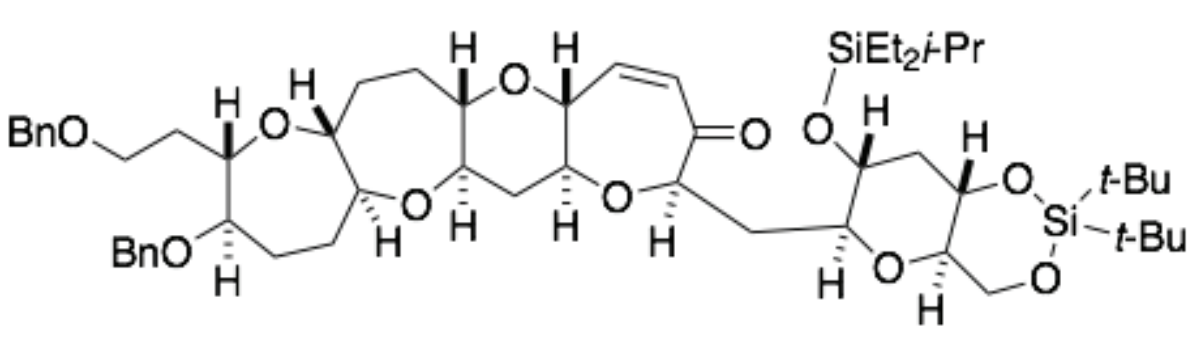

\section{C NMR spectrum of 38}

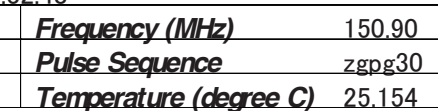

Pulse Sequence Date Stamp
Nucleus
Receiver Gain

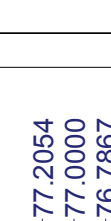
Origin
Solvent spect
CHLOROFORM-d \begin{tabular}{l|l}
\hline $13 \mathrm{C}$ & Number of Transients \\
\hline 193.26 & SW(cyclical) (Hz) \\
\hline
\end{tabular} 39062.50
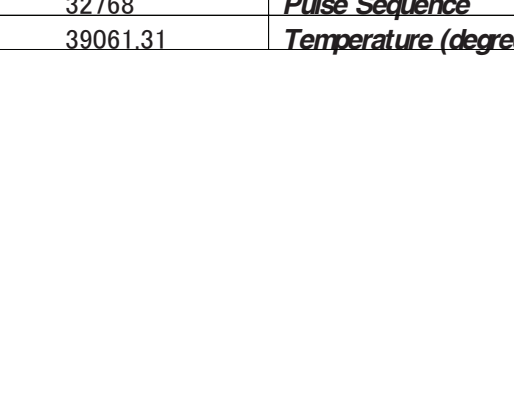
This report was created by ACD/NMR Processor Academic Edition. For more information go to www.acdlabs.com/nmrproc/

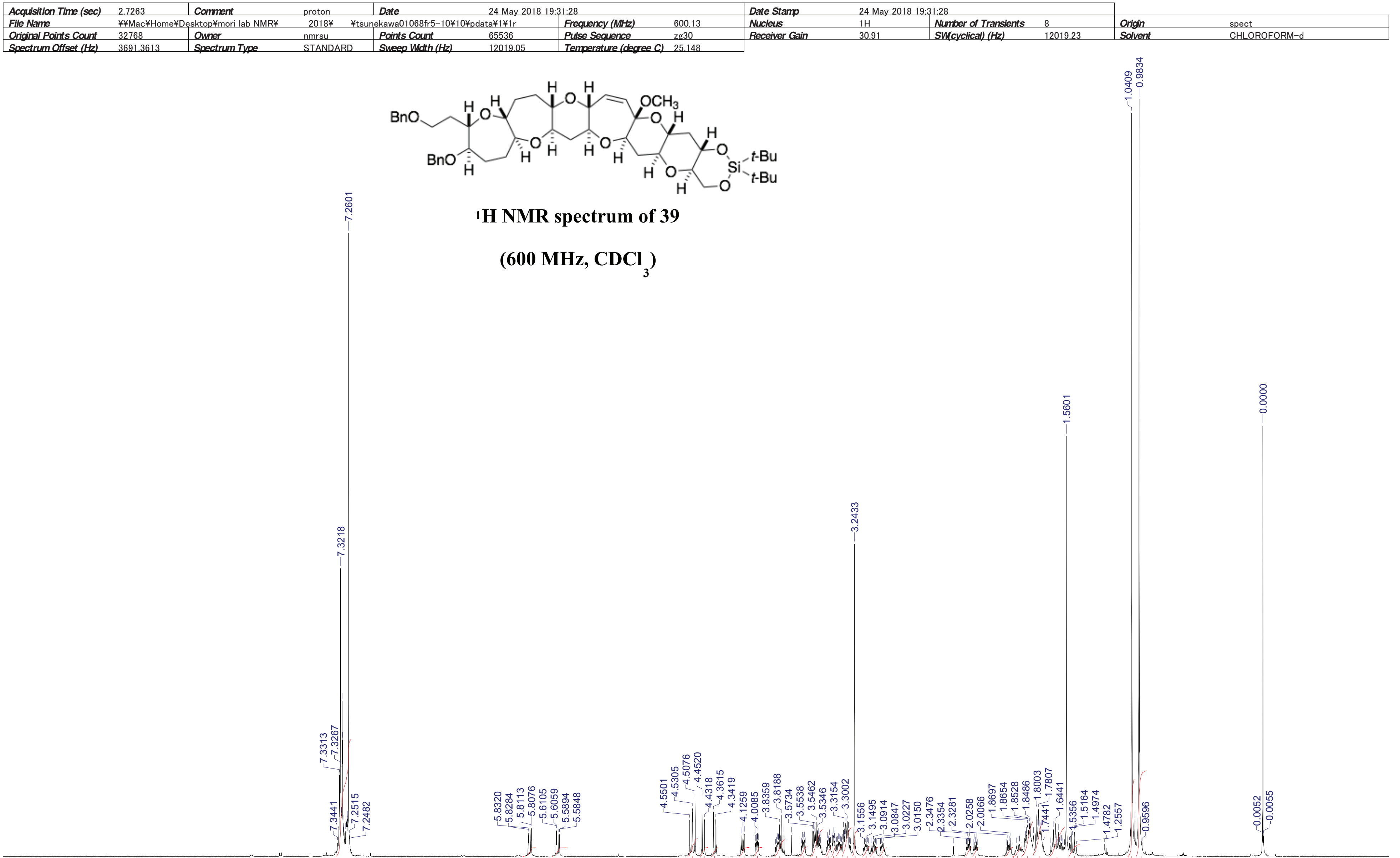

13.52

1.001 .00

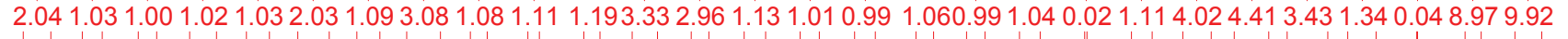




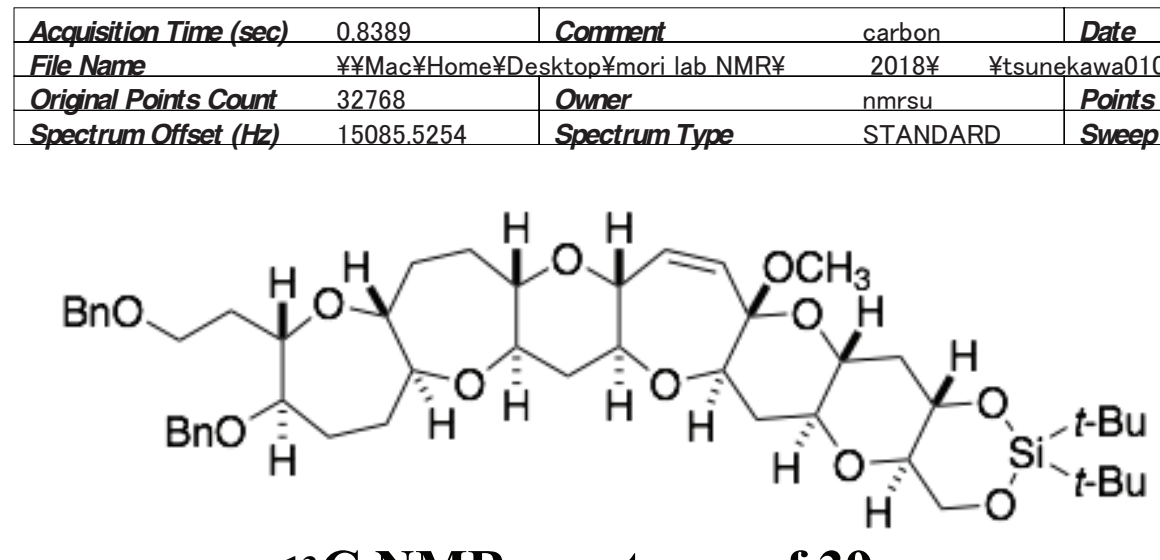

13C NMR spectrum of 39

(150 MHz, CDCl $)$

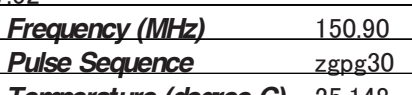

Pulse Sequence Date Stamo
Nucleus
Receiver Gai 24 May 2018 Number of Transient
SW(cyclical) (Hz) $\frac{128}{39062.50}$ Origin

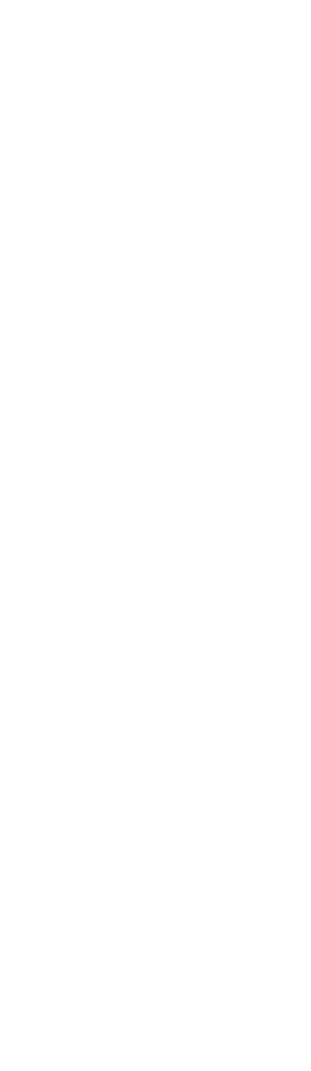

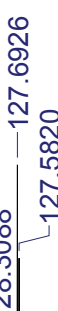

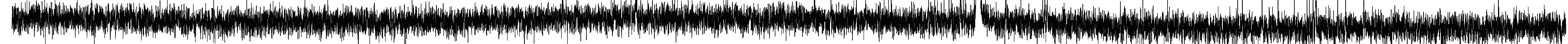

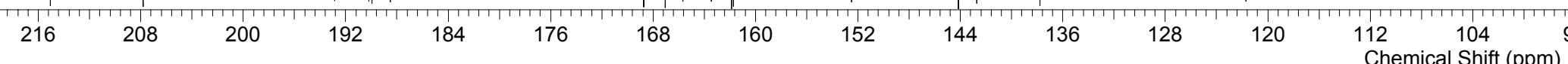

88

80

64

$56 \quad 48$

$48 \quad 40$

$32 \quad 24$ 
This report was created by ACD/NMR Processor Academic Edition. For more information go to www.acdlabs.com/nmrproc/

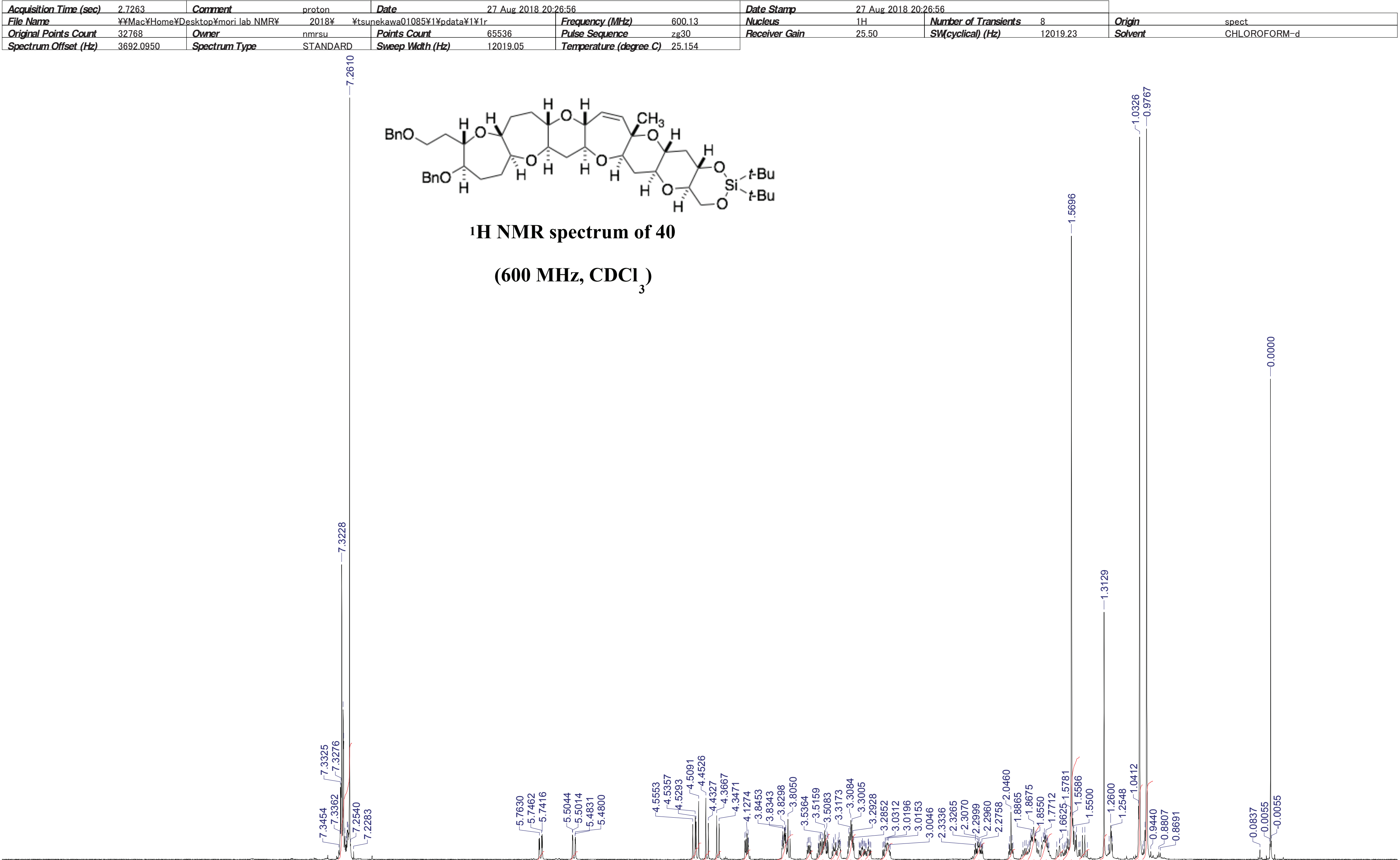

13.80

$\begin{array}{lll}0.93 & 0.91 & 1.94 \\ 0.99 & 0.961 .132 .931 .002 .970 .991 .022 .941 .080 .941 .901 .931 .201 .033 .993 .051 .110 .0112 .171 .172 .98 \\ 8.86 & 9.29\end{array}$ 
This report was created by ACD/NMR Processor Academic Edition. For more information go to www.acdlabs.com/nmrproc/

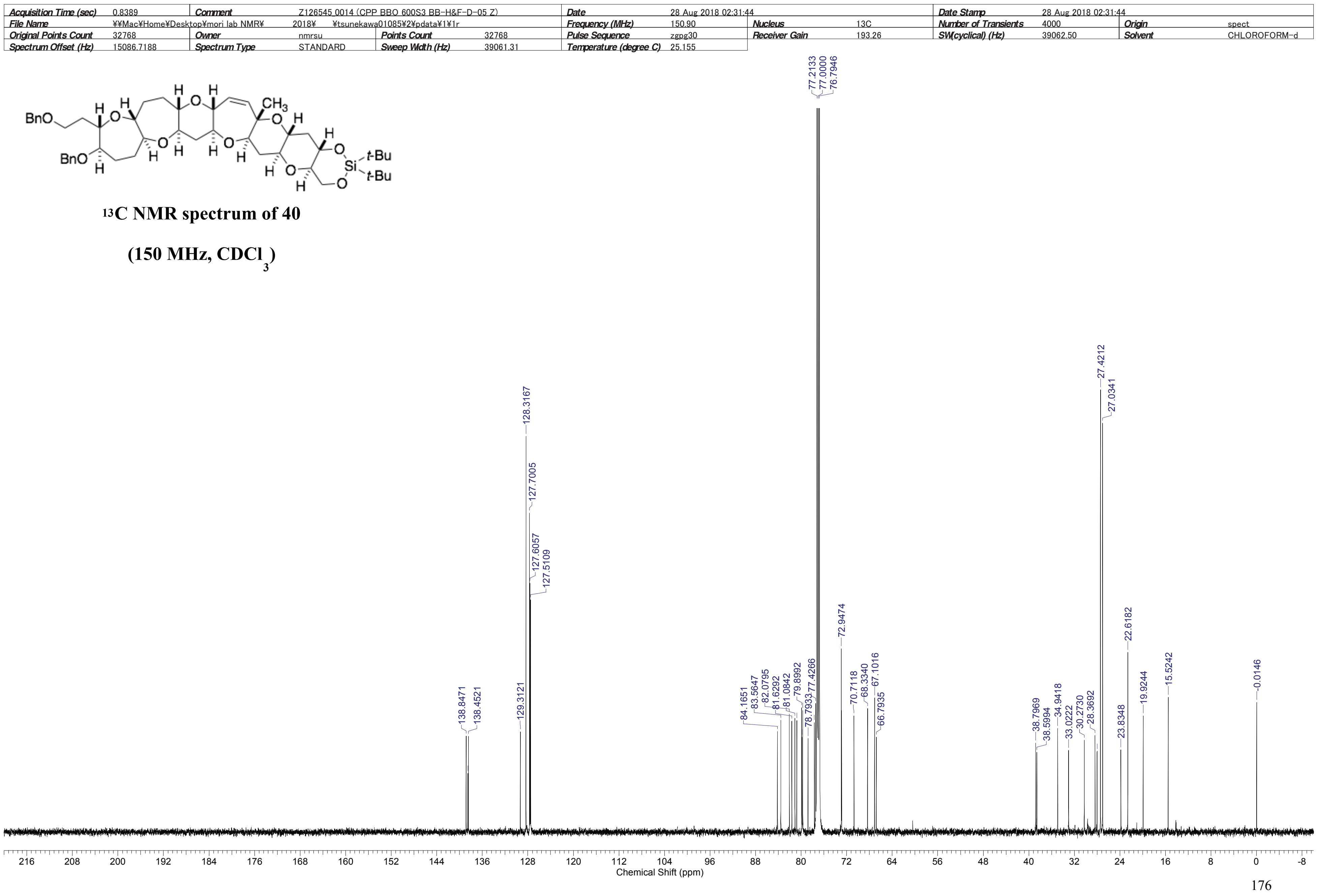


This report was created by ACD/NMR Processor Academic Edition. For more information go to www.acdlabs.com/nmrproc/

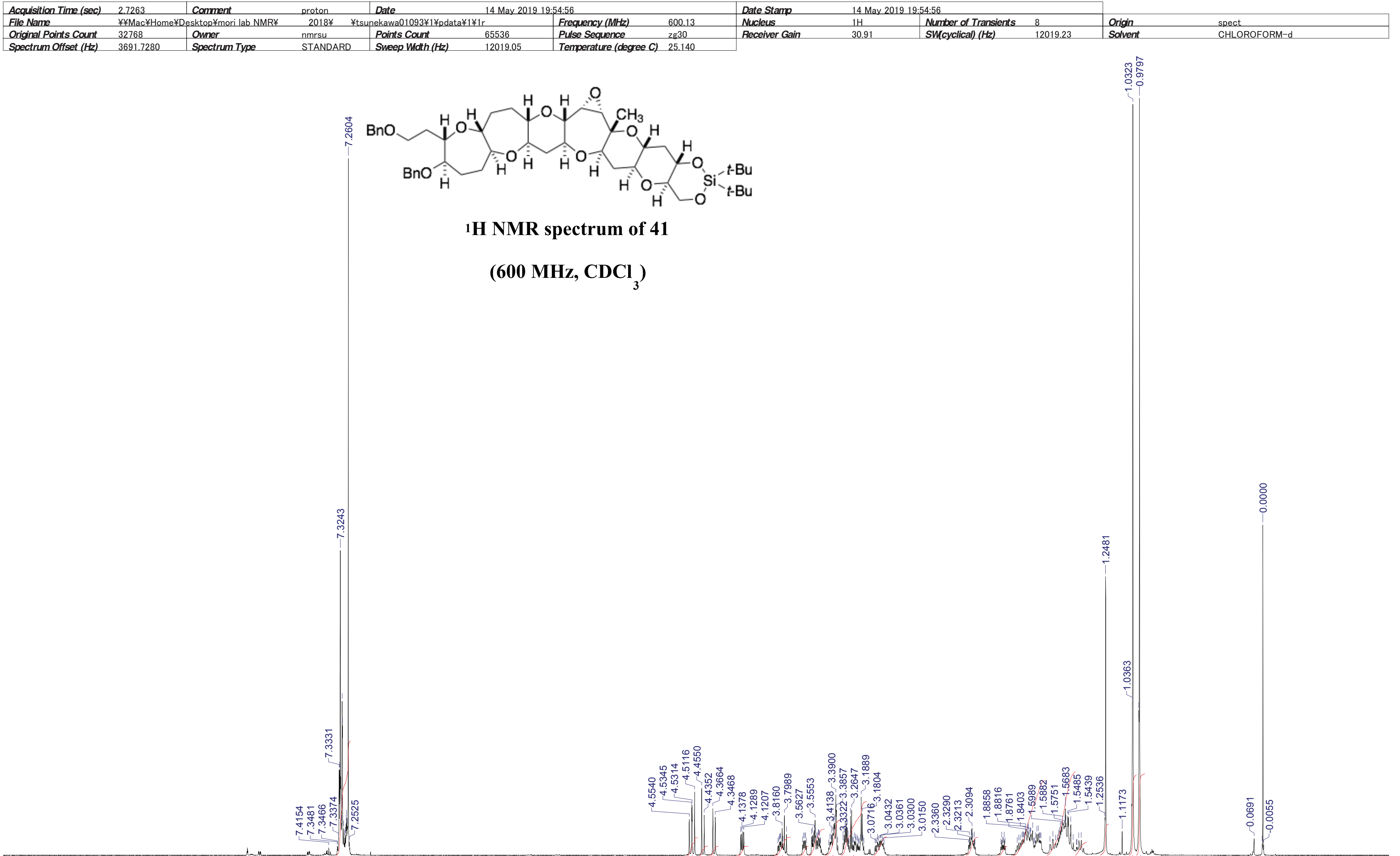

12.73

$1.951 .011 .001 .00 \quad 1.991 .012 .903 .925 .982 .06$

$2.00 \quad 1.00 \quad 8.32 \quad 9.321 .443 .478 .989 .08$ 
This report was created by ACD/NMR Processor Academic Edition. For more information go to www.acdlabs.com/nmrproc/

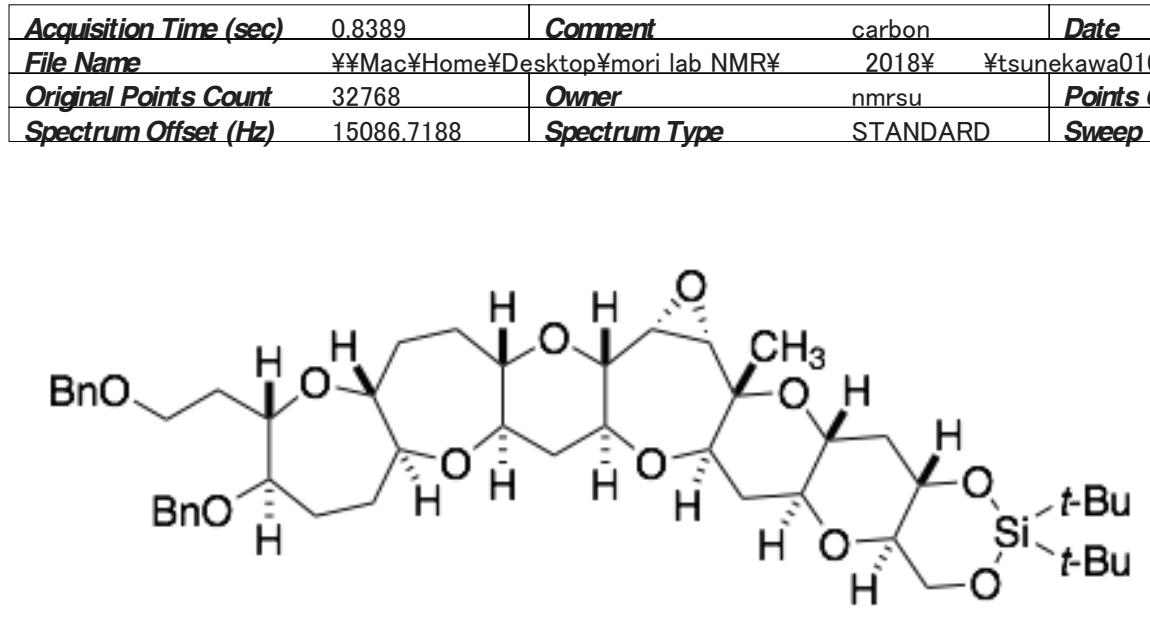

13C NMR spectrum of 41

$\left(150 \mathrm{MHz}, \mathrm{CDCl}_{3}\right)$

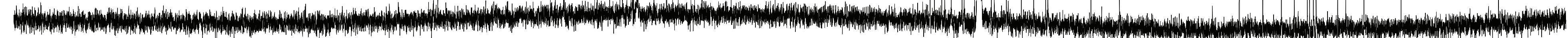

$\begin{array}{lllllllllllllll}216 & 208 & 200 & 192 & 184 & 176 & 168 & 160 & 152 & 144 & 136 & 128 & 120 & 112 & 104 \\ \text { Chemical Shift(ppm) }\end{array}$

88

$80 \quad 72 \quad 64$

48 


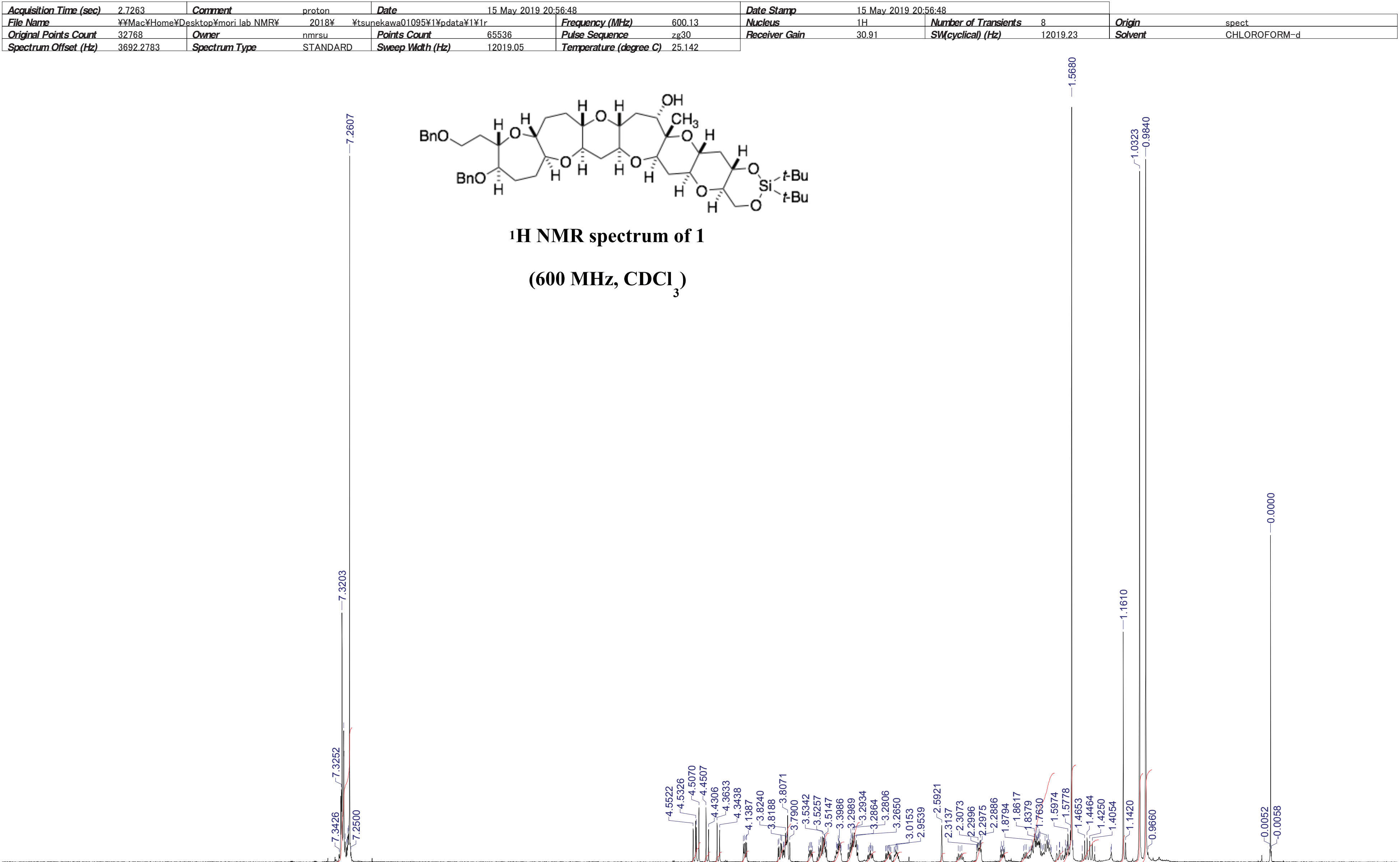




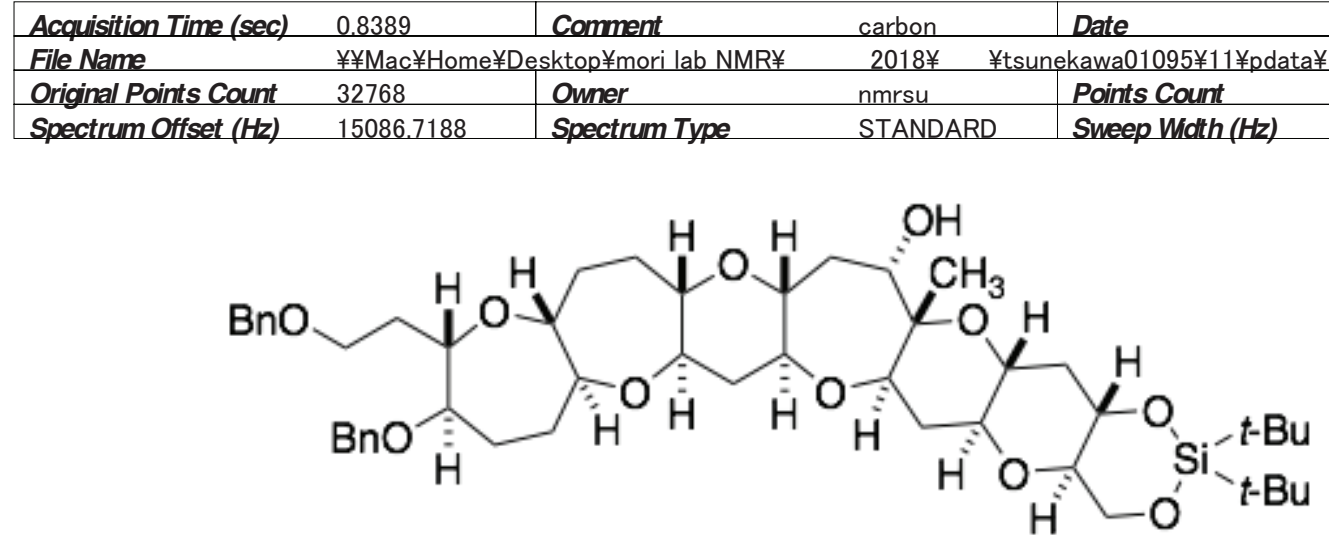

13C NMR spectrum of 1

(150 MHz, CDCl $)$ 